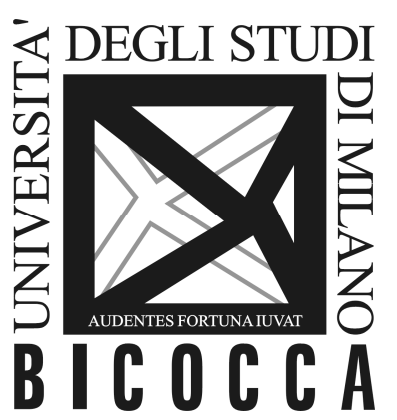

\author{
DEPARTMENT OF ECONOMICS, \\ MANAGEMENT AND STATISTICS \\ UNIVERSITY OF MILAN - BICOCCA
}

DEMS WORKING PAPER SERIES

\title{
Transitions and Political Stability in Autocracies. The Role of Public Perception
}

\author{
Mario Gilli, Yuan Li \\ No. 383 - July 2018
}

Dipartimento di Economia, Metodi Quantitativi e Strategie di Impresa

Università degli Studi di Milano - Bicocca

http://dems.unimib.it/ 


\title{
Transitions and Political Stability in Autocracies. The Role of Public Perception
}

\author{
Mario Gilli ${ }^{1} \quad \& \quad$ Yuan $\mathrm{Li}^{2}$
}

Version: July, 2018

\begin{abstract}
The literature on the functioning of autocracies has not analyzed the consequences of the fact that policies have multiple dimensions and that these dimensions are perceived with different bias by people. This fact is obviously more striking in autocracies where the public perception of policies' effects might be partially manipulated. We try to fill the gap. This paper makes three contributions to the literature on the functioning of autocratic regimes. First, we show that, may be counter-intuitively, both the probability of full efficient and full inefficient policies decrease as opacity increases, while the probability of partially efficient policies has the opposite behavior. This implies that the probability of efficient policies on different policy dimensions diverges as opacity increases, and this provides an explanation for the observed heterogeneity of policies within an autocracy. Second, the expected probability of a coup has a non monotone behavior w.r.t. opacity, so that at intermediate level an increment in opacity might actually increase the likelihood of a selectorate coup. Finally, also the expected probability of a citizens' revolt might have a non monotone behavior w.r.t. opacity, so that the likelihood of a revolt might actually increase as opacity increases. We conclude that the effect of bias in public perception of some policy dimension is non monotone on authoritarian regime stability. These results provide a reason to explain why transition periods are dangerous for a dictator.
\end{abstract}

JEL classifications: D02, H11, D74.

Keywords: Multidimensional policies, public perception, political stability.

\section{ACKNOWLEDGMENTS}

A first draft of this paper was written while Mario Gilli was visiting the Institute of East Asian Studies at the University of Duisburg-Essen. The hospitality and stimulating working environment are gratefully acknowledged. We thank Thomas Apolte, Sabine Carey, Mario Ferrero, Anna Pauls, Marco

\footnotetext{
${ }^{1}$ Department of Economics, University of Milan-Bicocca, Piazza dell'Ateneo Nuovo 1, 20126 Milan, Italy. Email: mario.gilli@unimib.it

${ }^{2}$ Institute of East-Asian Studies and Mercator School of Management, University of Duisburg-Essen. LE 738, Forsthausweg 2, Duisburg, Germany. Email: yuan.li@uni-due.de
} 
Serena and Ronald Wintrobe for their comments and insights, and especially we like to thank Piero Quatto for helpful suggestions. We also thank the participants at Games 2016, the European Public Choice Society Annual Meeting 2016 and the 2017 Workshop on Political Economy of Democracy and Dictatorship as well as the seminars' particpants at Freiburg University, Münster University and Bath University for helpful discussions. 


\section{INTRODUCTION}

The empirical and theoretical analysis has argued that autocracies looks similar yet they are actually different, and are different on multiple levels. One similarity is that autocracies like to control the diffusion of information so that policy-making is opaque and difficult to evaluate by the citizens. Nevertheless, they differ drastically in economic performances as well as in the stability of their ruling. ${ }^{3}$ The domestic political stability in autocracies can be threatened in two distinct ways: coups by the ruling elite and revolution by the citizens. In this paper, we try to make a structured analysis of the likelihood of coups or revolts in autocracies in terms of different public perception on different policy dimensions. Although the literature on autocracies has systematically increased, most of them have neglected the fact that policies have multiple dimensions and that these dimension are perceived in different ways and with different delays. We try to fill the gap by generalizing the available results on the functioning of autocratic regimes to the case of multi-dimensional policies with variant public perception. We can think about the following examples on how public perception on the effects of different policy dimensions are noisy. For example, while the tax burden of a change in income tax can be immediately perceived by the households, the effects of an environmental or anti-trust regulation may need years to fully display. Moreover, when government implements a price-fixing policy to control the price of some basic goods, the public can only perceive the effect of price, but not the quality of goods. Asymmetry in public perception of this kind generally exists in all political regimes once we consider multidimensional policies. However, the problem is more striking in autocracies due to the control of media and the widespread use of propaganda. ${ }^{4}$

There are many aspects where multidimensionality makes a significant difference w.r.t. to unidimensional policy model. In particular, as well known, multidimensional policies create many serious problem to voting models unless voters' preferences are significantly restricted, so that their differences are actually reduced to one dimension again. ${ }^{5}$ These problems can be avoided analyzing dictatorships, even if in a bargaining perspective multidimensionality is important,

\footnotetext{
${ }^{3}$ Some dictators are remarkably long-lasting, while others are surprisingly short-lived. For every long standing dynasty such as the one originating from Kim Il Sung in North Korea or Lee Kuan Yew in Singapore, there is a case of high turnover rate of dictatorial rulers, such as Haiti or Turkey or Argentina during the military dictatorship.

${ }^{4}$ In autocracies, leaders control the media to further their goals, typically, regime stability and policy implementation. However, media control is simply an instrument to try to manipulate public perception. In this paper we start directly from the fact that public perception of different policy dimensions are different.

${ }^{5}$ See Persson and Tabellini 2000.
} 
because concessions on one dimension may compensate full control on another dimension, as analyzed in Gilli, Li and Qian (2016) and in Gilli and Li (2017). It has long been recognized that controlling public information is essential for autocracies, however the research has never analyzed the differential effects of such distortion of public perception on different policy dimension and their complex effects on political stability. In particular it might seems that an increase in control of public perception should necessarily increase a regime stability, on the contrary we will show that this is not true once we take into consideration the differential effects on different policy dimensions. We will tackle this problem by analyzing how public perception on different policy dimensions affect the efficiency of government policies, which in turn affect the likelihood of coups or revolts. Let we stress that we are not considering the possibly contradictory links between authoritarian censorship and public beliefs, but the effect of differential public perception of different policy dimensions on the incentives on autocratic policy decisions, which in turn affect the likelihood of coups or revolts. In particular, the basic research question of this paper is to understand how the different levels of accountability on different policy dimensions of an autocratic leader affect a regime's stability.

The crucial innovation is the introduction of multidimensional policies and of an opacity parameter in a fully symmetric setting, where the only distinction between the two dimensions is given by a public signal which is fully informative with probability $1-\psi$ and fully non informative with probability $\psi$, so that $\psi$ is a simple clear measure of the opacity of the public perception of a policy dimension. This paper makes three contributions to the literature on the functioning of autocratic regimes. As a preliminary step, we generalize the available results on accountability in autocratic polities to the case of multidimensional policies. This result has three important corollaries. First, we show that, may be counter-intuitively, both the probability of full efficient and full inefficient policies decrease as opacity increases, while the probability of partially efficient policies has the opposite behavior. This implies that the probability of efficient policies on different policy dimensions diverges as opacity increases, providing an explanation for the observed heterogeneity of policies within an autocracy. Second, the expected probability of a coup has a non monotone behavior with regard to opacity, so that an increase in opacity might actually increase the likelihood of a coup. Finally, also the expected probability of a citizens' revolt might have a non monotone behavior with regard to opacity, so that also the likelihood of a revolt might actually increase as opacity increases. We conclude that the effect of public perception bias toward different policy dimensions on the stability of authoritarian regime is non monotone. These results can explain why transitional periods towards or from more liberal 
public information are dangerous for a dictator.

The paper proceeds as follows. Section 2 presents the related literature, section 3 provides the model. Section 4 is the core of the paper where we provide the equilibrium outcomes and discuss the relations between equilibrium policies, public perception and the likelihood of coups or revolts. Section 5 provides two case studies, while section 6 concludes. All the calculations are in appendix.

\section{RELATED LITERATURE}

Our paper builds on a growing literature on the inner working of authoritarian political institutions. In this literature, all dictators share the same primary goal: to hold on to office at all costs, because failing to do so will result in imprisonment, exile, or execution. The main threats to the dictators' political survival are the loss of support among key supporters and revolutionary challenges that can topple the political system. Therefore, some researchers focus on coups of the selectorate (Bueno de Mesquita et al. 2003, Besley and Kudamatsu 2008, Svolik 2009 and 2012, Egorov and Sonin 2011, Gilli and Li 2013), while some others focus on revolution of the citizens (Acemoglu and Robinson 2006; Smith 2008, Bueno de Mesquita and Smith 2009 and 2010, Svolik 2013; Aidt and Jensen 2014; Gilli and Li 2014 and 2015; Dorsch and Maarek 2015). We follow the modelling strategy of Besley and Kudamatsu 2008 and Gilli and Li 2013, 2014 and 2015, modelling autocratic politics as an incomplete information game. In this framework, the dictator's incentive to prevent coups and revolutions works as an incentivizing mechanism and shapes a wide variety of economic and political outcomes in autocratic regimes. Based on this logic, there are two types of accountability mechanisms in autocracies as argued by Gandhi and Przeworski (2006). We combine them in one model, connecting efficient policy outcomes in autocracies to two de facto power parameters, the effective size of the selectorate $(\phi)$ and the inverse of the cost of revolution $(\zeta)$. The crucial innovation of this paper is that we assume policies are multi-dimensional, whose effects are differently perceived by the public. The opacity in public perception is modelled as the probability $(\psi)$ of not observing the real effect of a policy dimension. The effect of $\psi$ on the likelihood of coups and revolution is the focus of the paper.

Our paper is a further step on the research program of transparency and the functioning of autocracies. Smith 2008, Bueno de Mesquita and Smith 2009 and 2010 consider how the leaders can manipulate free press and transparency, what they call coordination goods, to reduce the 
citizens' desire for revolution. In their view there is a trade-off between opacity and productivity that constrain the leader's choice of the optimal level of transparency. Guriev and Tresiman 2016 focus on the trade-off between information manipulation and repression, where incompetent leaders might survive by manipulating information instead of using violent repression which would clearly reveal they are incompetent. Whereas in all the above works, propaganda and public perception are endogenous, we assume that the amount of manipulation of public perception is exogenous, and we analyze how it relates to the endogenous probability of coups and revolutions when policies have multiple dimensions. Hence, our analysis are complementary. Furthermore, in the above works, public perception has monotonic effects on the efficiency of government policies and regime stability, whereas our paper suggests that these effects might be non monotonic, thus innovating on the explanation of why transitional periods are dangerous for regime stability.

A different approach to public perception in autocracies is provided by Reinikka and Svensson 2005, Egorov et al. 2009, Lorentzen 2014 and Qin et al. 2014. All these works start from a fact that many autocracies allow partially free media despite apparent risks. The explanation is that autocracies face a trade-off between regime stability and more effective policy implementation. Partially free media allows autocracies to better supervise lower-level bureaucrats and thus to improve the quality of governance. Our paper provides an alternative explanation to this. We show that an autocracies might allow partially free media because intermediate opacity would minimize the probability of a citizens' revolt.

From a more general point of view, many papers have analyzed the effect of change in transparency on accountability within a principal-agent model. ${ }^{6}$ The starting point of this literature is the Holmstrom Principle (Holmstrom 1979) which states that more transparency makes both the principal and the agent better off. The subsequent works have tried to understand when Holmstrom principle doesn't hold. In dynamic models with incomplete contracts (often called career concerns models $)^{7}$, more transparency can create an incentive for the agent to behave in a conformist way, which might be damaging to the principal. Our model is different, because in our model it is the variant public perception on different policy dimensions that induces the divergence of agent's choice on these dimensions which, in turn, induces the non monotonicity in the principals' behavior.

\footnotetext{
${ }^{6}$ In our model, the leader is the agent, while the selectorate and the citizens are the principals.

${ }^{7}$ See, for example, Dewatripont et al. 1999, Holmstrom 1999, Prat 2005.
} 


\section{THE MODEL}

The model we use is a generalization of the model in Gilli and Li 2015, where policies have two dimensions that differ in the public perception of their effects. Thus, we have a two-period political-agency model with incomplete information played by three protagonists: the dictator, the selectorate, and the citizens. Contrary to standard political-agency models for democracies (Berganze 2000, Besley 2006, Maskin and Tirole 2004), there is no regular general election; hence, the dictators' term might be indeterminate. However, dictators can be removed from office by the selectorate through a coup or by the citizens through a revolution. Revolutions are defined as popular revolts whose goal is a permanent change in the distribution of a country's wealth. Coups, instead, are defined as a forced resignation of the dictator without any transformation of the political regime. A coup does not change the distribution of a country's wealth but instead changes the composition of the selectorate and the identity of the dictator. Hence, the threat of a revolution is different from the threat of a coup. Thus, we introduce two separate conflict technologies, one for coups, and one for revolutions. Then, the dictators face two basic problems of governance: first, they need the cooperation of the selectorate and, second, they need to avoid a revolution. When dictators face credible threats by citizens or by the selectorate, they are pressed to choose efficient policies instead of appropriating private benefits. However, dictators differ in their incentives to implement efficient policies.

We formalize these ideas as follows. In each period $t=1,2$, there are three players: the dictator $(L)$ (female), the selectorate $(S)$ (male) and the citizens $(Z)$ (plural). In the first period, the three players play sequentially, whereas in the second period only the dictator has a possible choice, if she has not been removed by a successful revolution. The dictator can be one of two types, either congruent or non-congruent, $T \in\{C, N\}$, with probability $\pi$ of being congruent, and with different payoffs, as explained below. At time $t \in\{1,2\}$ the dictator is privately informed of the true state of nature $\theta_{t}=\left(\theta_{t}^{1}, \theta_{t}^{2}\right) \in\{0,1\} \times\{0,1\}$ and has to make a discrete policy choice denoted by $e_{t}=\left(e_{t}^{1}, e_{t}^{2}\right) \in\{0,1\} \times\{0,1\}$. Public interest requires the dictator to match the true state of nature, i.e. to choose an efficient policy, but this would also mean that the non-congruent dictator foregoes her private benefits. The public payoff from the 
policy is

$$
\left\{\begin{array}{ll}
\Delta^{1}+\Delta^{2} & \text { if } e_{t}^{1}=\theta_{t}^{1} \& e_{t}^{2}=\theta_{t}^{2} \\
\Delta^{1}+\widetilde{\Delta}^{2} & \text { if } e_{t}^{1}=\theta_{t}^{1} \& e_{t}^{2} \neq \theta_{t}^{2} \\
\Delta^{2} & \text { if } e_{t}^{1} \neq \theta_{t}^{1} \& e_{t}^{2}=\theta_{t}^{2} \\
\widetilde{\Delta}^{2} & \text { if } e_{t}^{1} \neq \theta_{t}^{1} \& e_{t}^{2} \neq \theta_{t}^{2}
\end{array} \quad \text { where } \quad \widetilde{\Delta}^{2}= \begin{cases}\Delta^{2} & \text { prob } \psi \\
0 & \text { prob } 1-\psi\end{cases}\right.
$$

Hence, the efficient policy produces a public good, however the effects of the second policy dimension are perceived noisily: with probability $1-\psi$ the effects are correctly perceived, while with probability $\psi$ there is misperception in the sense that people believe the choice was efficient even if it wasn't. Hence $\psi \in[0,1]$ is our measure of opacity in public perception. ${ }^{8}$ The noncongruent dictator receives a private benefit $r_{t}^{i}$ from picking $e_{t}^{i} \neq \theta_{t}^{i}$, where $r_{t}^{1}$ is stochastically independent from $r_{t}^{2}$ and $r_{t}^{i}$ is drawn according to a continuous cumulative distribution function $G^{i}\left(r_{t}^{i}\right)$ with $E\left(r_{t}^{i}\right)=\bar{r}^{i}, G^{i}\left(\Delta^{i}\right)=0$, and $G^{i}\left(r_{t}^{i}\right)>0$ for $r_{t}^{i}>\Delta^{i}$. The congruent dictator obtains no private benefit from selecting $e_{t}^{i} \neq \theta_{t}^{i}$. The interpretation of a dictator's type can be quite broad. A non-congruent type can be an incompetent dictator who finds it costly to adopt an efficient policy. Or she can be ideological, pursuing her ideological policy notwithstanding the actual situation. Whatever the interpretation, the role of the type is to provide an opportunity for the dictator to credibly commit to a specific policy through her reputation. This allows us to model the idea that economic policies might be wrong not because of ignorance or for cultural or technological reasons, but because of political incentives. To gain the loyalty of the selectorate, the dictator pays patronage to the selectorate.

We suppose that the patronage is funded through the distribution of resources, $X$. From this patronage, the citizens obtain 0 and the selectorate gains $\frac{X}{\phi},{ }^{9}$ where $\phi \in[0,1]$ is a measure of the effective size of the selectorate. Thus, the selectorate obtains his utility from the dictator's policy and then he decides whether to support or remove her. If the selectorate support the dictator, then the dictator still holds office in the subsequent period, otherwise the dictator is ousted from power, as a dictator with no basis of support cannot survive. When the incumbent autocrat is ousted from power, a new ruler will replace her and form a new selectorate of size $\phi$. Note that the selectorate's removal of the incumbent dictator does not change the basic social order and

\footnotetext{
${ }^{8}$ Note that many may argue that public perception of policy dimensions is endogenous and in full control of dictators. Our point is that most political change take place at critical juncture of history, when it is plausible to assume that dictators are not able to fully control the rapid changes in public perception. Under such situation, there might be slow exogenous change towards more liberal flows of information or attempts of increasing autocratic control of public perception that have exogenous lags.

${ }^{9}$ Naturally, this is just normalization.
} 
governmental apparatus in the country, it is just a coup d'état, not a revolution. Hence, when the incumbent dictator is ousted from power by a coup, a new dictator will rise with the support of a new selectorate. We assume that the effective size of the new selectorate remains the same because there is no regime change. ${ }^{10}$ The new dictator will randomly select the members of the new selectorate from the pool of the population. Thus, each member of the old selectorate has a probability $\phi$ of being included in the new selectorate.

After the selectorate's choice, the citizens obtain their utility from the dictator's policy and the selectorate's choice, and choose whether to revolt.

The game then proceeds to the second period, in one of three possible states: no revolt, successful revolution and unsuccessful revolution. The dictator's possible actions are different in these states. We assume that a revolution would eliminate the possibility of making economic policies, because the unique choice for the dictator is to fight the revolt. Hence, a revolution is actually a conflict on the division of the given resources, $X$. The payoffs implied by the secondperiod choices are realized and the game ends. If there is no revolution, then the dictator remains in power and her type is unchanged. She observes the second period nature's choice $\theta_{2} \in\{0,1\}$ and again has to make a discrete policy choice denoted by $e_{2} \in\{0,1\}$. The players' second-period payoffs are then determined as in the first period following this policy choice. If the revolution succeeds, the citizens will receive the selectorate's patronage net of the revolution's cost $\eta, \frac{X-\eta}{1-\phi}$. The dictator and the selectorate will obtain a large negative payoff, $-D$, because they are ousted from power and fear for their life. Again, both these payoffs are realized at the beginning of the second period. If the revolution fails, the citizens obtain 0 , and the dictator and the selectorate obtain the patronage net of the repression $\operatorname{costs} k, \frac{X-k}{\phi} .^{11}$

We assume a simple conflict technology for revolts: the revolution succeeds with probability of $1-\phi$, i.e., the probability of success is linearly increasing with the effective size of the citizens. Hence, after a revolution, the citizens' expected payoff is $X-\eta$, whereas the dictator's and the selectorate's expected payoffs are $X-k-D+\phi D$. We assume $D$ is sufficiently large that the dictator and the selectorate will always want to avoid taking the chance of a revolution, if possible. Moreover, to simplify calculations, we assume the dictator's and the selectorate's expected payoff is equal to 0 . A negative or a small positive second-period expected payoff would

\footnotetext{
${ }^{10}$ This hypothesis could be relaxed without changing our main results, as we show in Gilli and Li (2013). However, it would introduce a needless complication.

${ }^{11}$ Introducing $k$ is just for symmetry; if it is costly for the citizens to initiate a revolution, it should also be costly for the dictator to repress it. However, $k$ will not affect the normalization of the expected payoffs of the dictator and the selectorate.
} 
make calculations more complex without adding any meaningful insight. This assumption is the simplest way of modelling the idea that both the dictator's and the selectorate's most important aim is to avoid a revolution that would challenge their political regime, whenever possible. Thus, we model the revolution in the most simple manner as a constraint on the dictator's and the selectorate's behavior, as argued by Acemoglu and Robinson (2006). Moreover, note that in these types of models, the second period simply has the role of providing forward incentives to the players' first period choices. They are not aimed at analyzing the transition from autocracies to different political regimes.

In the model, a crucial role is played by the effective size of the selectorate $\phi$ and by the inverse of the cost of revolution $\zeta=(\eta)^{-1}$. We claim that these parameters actually capture the de facto political power of the selectorate and of the citizens in an autocratic regime. Let consider $\phi$ : this parameter is a measure of the probability of being reappointed in the selectorate after a coup and it is the complement of the probability of a successful citizens' revolt, hence the higher $\phi$, the less risky is a coup. On the other hand $\eta$ is a measure of the certain cost of a revolt, hence the higher these costs the smaller the incentives to revolt. In order to deal directly with the citizens' incentive to revolt, we use $\zeta=(\eta)^{-1}$. Finally, suppose that $\eta \simeq \widetilde{\eta} \in[\varepsilon, \infty]$, so that w.l.g. we might assume that $(\zeta, \phi) \in[0,1] \times[0,1]$.

To sum up, the timing of the model is as follows:

1. Nature determines $\left(\theta_{1}, r_{1}\right) \in\{0,1\} \times\{0,1\} \times\left[\Delta^{1}, \infty\right) \times\left[\Delta^{2}, \infty\right)$ and the type of dictator $T \in$ $\{C, N\}$. These five random variables are stochastically independent and their realization is private information of the dictator.

2. Type $T$ dictator chooses a policy, and the payoffs for each player in period one are realized. The probability of a congruent choice of type $T$ dictator on dimension $i$ at period $t$ is denoted by policy in period $t$ is denoted by $\lambda_{t}^{T, i}\left(\theta_{t}^{i}, r_{t}^{i}\right)=\mathbb{P}\left(e_{t}^{i}=\theta_{t}^{i} \mid \theta_{t}^{i}, r_{t}^{i}\right)$. Moreover, let define

$$
\bar{\lambda}_{t}^{T, i}=\int_{-\infty}^{\infty} \lambda_{t}^{T, i}\left(\theta_{t}^{i}, r_{t}^{i}\right) d G^{i}\left(r_{t}^{i}\right), \text { with } T \in\{C, N C\}, i \in\{1,2\}, t \in\{1,2\} .
$$

3. The selectorate observes the realization $\delta$ of $\Delta^{1}$ and $\widetilde{\Delta}^{2}$, and on the basis of this information decides whether to retain the incumbent dictator. The probability of a coup is denoted by $\rho:\left\{0, \Delta^{1}\right\} \times\left\{0, \Delta^{2}\right\} \longmapsto[0,1]$. A coup succeeds with certainty.

4. If the incumbent dictator is ousted from power, a new dictator will enter office and she will be congruent with a probability of $\pi$. The new dictator will form her own selectorate and the members of the selectorate who deposed the previous dictator will have a probability 


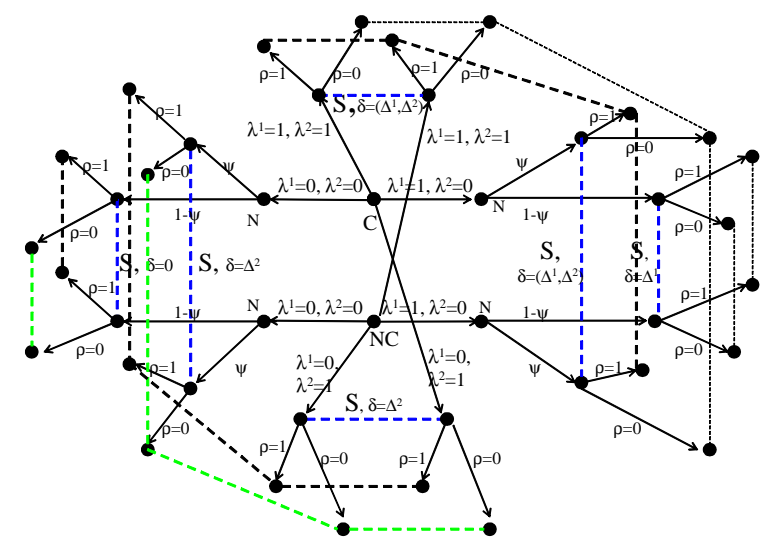

FIG. 1 The first stage of the game.

$\phi$ of being included in the new one.

5. The citizens observe the choice implemented by the selectorate, $\widehat{\rho} \in\{0,1\}$, and the effect of the policy chosen by the dictator but not her type. Based on this information, they decide whether to initiate a revolution. The probability of a revolution is denoted by $\alpha:\left\{0, \Delta^{1}, \Delta^{2}, \Delta^{1}+\Delta^{2}\right\} \times\{0,1\} \longmapsto[0,1]$. A revolution succeeds with probability $1-\not \phi$ and fails with probability $\phi$.

6. The game enters the second period and nature determines $\left(\theta_{2}, r_{2}\right)$. In the second period, there are three possible states:

(a) No revolution: the dictator remains in power and her type is unchanged. She observes nature's choice and chooses a policy according to her type. The payoffs are realized and the game ends.

(b) Successful revolution: the dictator and the selectorate are removed from power and obtain a large negative payoff $-D$, whereas the citizens divide the country's wealth $X$, receiving a payoff $\frac{X-\eta}{1-\phi}$ net of the revolution's costs $\eta$. These payoffs are realized and the game ends.

(c) Failed revolution: the dictator and the selectorate divide the country's wealth $X$, receiving a payoff $\frac{X-k}{\phi}$ net of the repression costs $k$, whereas the citizens obtain a 0 payoff. These payoffs are realized and the game ends.

The first-stage game structure is reported in Figure 1 without the possible actions of the citizens, to simplify the presentation: 
All players' utilities are linear in their consumption with a unitary discount factor. Their formal expression is reported in the Appendix.

The notation used is summarized in the following table:

Table 1. Definition of Symbols

\begin{tabular}{|c|c|}
\hline SYMBOL & DEFINITIONS \\
\hline & PLAYERS \\
\hline$L$ & incumbent dictator \\
\hline$T \in\{C, N\}$ & type of the incumbent dictator with $\operatorname{Pr}\{T=C\}=\pi$ \\
\hline$S$ & Selectorate \\
\hline \multirow[t]{2}{*}{$Z$} & Citizens \\
\hline & EXOGENOUS VARIABLES \\
\hline$\theta_{t}^{i} \in\{0,1\}$ & state of nature at time $t$ for policy $i \in\{F, E\}$ \\
\hline$r_{t}^{i} \sim G^{i}$ & private rent the dictator can extract at time $t$ for policy $i \in\{F, E\}$ \\
\hline$\delta \in\left\{0, \Delta^{1}\right\} \times\left\{0, \Delta^{2}\right\}$ & payoffs from dimension 1 and 2 of public policies \\
\hline$\widetilde{\Delta}^{2}$ & random payoff from an inefficient policy 2 \\
\hline$X$ & exogenous revenue of the country \\
\hline$k$ & the cost of repression \\
\hline$\psi \in[0,1]$ & probability of distortion of the signal of policy 2 \\
\hline$\phi \in[0,1]$ & de facto power of the selectorate \\
\hline \multirow[t]{2}{*}{$\zeta \in[0,1]$} & de facto power of citizens \\
\hline & ENDOGENOUS VARIABLES \\
\hline$\left(e_{t}^{1}, e_{t}^{2}\right)$ & policies actually implemented at time $t$ on dimensions 1,2 \\
\hline $\bar{\lambda}_{t}^{T, i} \in[0,1]$ & probability of efficient policy at time $t$ on dimension $i$ for type $T$ \\
\hline$\rho(\delta) \in[0,1]$ & probability of a coup \\
\hline \multirow[t]{2}{*}{$\alpha(\delta, \rho) \in[0,1]$} & probability of a citizens' revolt \\
\hline & PAYOFFS \\
\hline$U^{T / Z / S}(\lambda, \alpha, \rho)$ & first-period utility of type $T$ dictator/selectorate/citizens \\
\hline$V^{T / S / Z}$ & expected continuation payoff of type $T$ dictator/selectorate/citizens \\
\hline
\end{tabular}

Thus, our aim is to use this model to explain

1. the probability of a dictator's congruent policy choice $\left(\bar{\lambda}^{1}, \bar{\lambda}^{1}\right)$

2. the probability of a coup $\rho$ 
3. the probability of a revolution $\alpha$

in terms of a crucial parameter, the bias in the public perception of dimension 2 of policy choices $\psi$.

\section{POLICY CHOICES AND OPACITY}

To solve the game we use Sequential equilibrium (SE) as equilibrium concept. Moreover, to refine out of equilibrium beliefs we use a strong version of Forward Induction in the sense that any deviations from full efficiency is interpreted as due to the non congruent leader. In this way, we find a unique equilibrium for each possible combinations of citizens and selectorate de facto power $(\zeta, \phi)$ and we are able to characterize the properties of these equilibria as a function of the bias in the public perception of dimension 2 of policy choices $\psi{ }^{12}$

The logic of the players' equilibrium strategic behavior is similar to what we explained in Gilli and Li 2015, hence we just illustrate the possible equilibrium outcomes that depends on the players' de facto power. ${ }^{13}$ We interpret the outcomes of these equilibria as different autocratic regimes according to the dictator's incentives for choosing an efficient policy. There are four possible equilibrium outcomes when we consider the non-congruent leader's ${ }^{14}$ policy choices and the consequent reaction by selectorate and by citizens: ${ }^{15}$

1. a Kleptocratic outcome, where the leader's inefficient policies don't trigger any reaction by the selectorate and the citizens;

2. a Roving Bandit outcome, where the leader's inefficient policies trigger a coup by the selectorate and/or a revolt by the citizens;

3. a Partially Efficient outcome, where the leader choose an efficient policy only on the full observable policy, risking a coup by the selectorate and/or a revolt by the citizens because of the inefficient choice on the partially observable policy;

4. a Full Efficient outcome, where the leader choose an efficient policy on both dimensions, to minimize the risk of a coup by the selectorate and/or a revolt by the citizens.

\footnotetext{
${ }^{12}$ Note that we consider pure strategy equilibria whenever possible, but since the non-congruent dictator's choice will depend on the realization of her random private rent not observed by the selectorate and the citizens, from their point of view the dictator's choice will be random. Similarly, since the selectorate and the citizens choices will depend on $\delta$, whose realization is random, the actual players' choice will be random.

${ }^{13}$ In the Appendix we report all the detailed calculations required to derive the Sequential equilibria of our model.

${ }^{14}$ We focus on the choices facing the non-congruent dictator because they are the actual relevant policy choices to characterize the type of an autocratic polity.

${ }^{15}$ Note that a single equilibrium is associated to multiple outcomes with well defined probabilities.
} 
Related to these four possible equilibrium outcomes, there are four possible political autocratic regimes with different observable characteristics. The following table present a global synthetic qualitative view of the combination of $\phi$ and $\zeta$ that give rise to the different expected equilibrium outcomes, with their observable characteristics. 


\begin{tabular}{|c|c|c|}
\hline & $\phi$ small & $\phi$ big \\
\hline$\zeta$ small & $\begin{array}{c}\text { Kleptocratic A. } \\
\text { Kleptocratic outcome } \\
E\left(\bar{\lambda}_{1}^{*}\right)=0 \\
E\left(\bar{\lambda}_{1}^{2}\right)^{*}=0 \\
E\left[\rho^{*}\right]=1 \\
E\left[\alpha^{*}\right]=0\end{array}$ & $\begin{array}{c}\text { Semi Efficient A. } \\
\left\{\begin{array}{c}\text { Roving Bandit outcome } \\
\text { Part. Efficient outcome } \\
\text { Full Efficient outcome } \\
E\left(\bar{\lambda}_{1}^{1}\right)^{*}=F E+P E \\
E\left(\bar{\lambda}_{1}^{2}\right)^{*}=P E \\
E\left[\rho^{*}\right]=F I+(1-\psi) P E \\
E\left[\alpha^{*}\right]=0\end{array}\right.\end{array}$ \\
\hline interm & $\begin{array}{c}\text { Semi Efficient A. } \\
\left\{\begin{array}{c}\text { Roving Bandit outcome } \\
\text { Part. Efficient outcome } \\
\text { Full Efficient outcome } \\
E\left(\bar{\lambda}_{1}^{1}\right)^{*}=F E+P E \\
E\left(\bar{\lambda}_{1}^{2}\right)^{*}=F E+\psi P E \\
E\left[\rho^{*}\right]=F I+(1-\psi) P E \\
E\left[\alpha^{*}\right]=0\end{array}\right.\end{array}$ & $\begin{array}{c}\text { Semi Efficient A. } \\
\left\{\begin{array}{c}\text { Roving Bandit outcome } \\
\text { Part. Efficient outcome } \\
\text { Full Efficient outcome } \\
E\left(\bar{\lambda}_{1}^{1}\right)^{*}=F E+P E \\
E\left(\bar{\lambda}_{1}^{2}\right)^{*}=F E+\psi P E \\
E\left[\rho^{*}\right]=F I+(1-\psi) P E \\
E\left[\alpha^{*}\right]=0\end{array}\right.\end{array}$ \\
\hline interm & $\begin{array}{l}\text { Mixed Semi Efficient A. } \\
\left\{\begin{array}{c}\text { Roving Bandit outcome } \\
\text { Part. Efficient outcome } \\
\text { Full Efficient outcome }\end{array}\right. \\
E\left(\bar{\lambda}_{1}^{1}\right)^{*}=M F E+M P E \\
E\left(\bar{\lambda}_{1}^{2}\right)^{*}=M F E+\psi M P E \\
E\left[\rho^{*}\right]=[M F I+(1-\psi) M P E] \bar{\rho} \\
E\left[\alpha^{*}\right]=\bar{\alpha}\end{array}$ & $\begin{array}{l}\text { Mixed Semi Efficient A. } \\
\left\{\begin{array}{c}\text { Roving Bandit outcome } \\
\text { Part. Efficient outcome } \\
\text { Full Efficient outcome }\end{array}\right. \\
E\left(\bar{\lambda}_{1}^{1}\right)^{*}=M F E+M P E \\
E\left(\bar{\lambda}_{1}^{2}\right)^{*}=M F E+\psi M P E \\
E\left[\rho^{*}\right]=[M F I+(1-\psi) M P E] \bar{\rho} \\
E\left[\alpha^{*}\right]=\bar{\alpha}\end{array}$ \\
\hline$\zeta$ big & $\begin{array}{c}\text { Failed State A. } \\
\text { Roving Bandit outcome } \\
E\left(\bar{\lambda}_{1}^{1}\right)^{*}=E\left(\bar{\lambda}_{1}^{2}\right)^{*}=0 \\
E\left[\rho^{*}\right] \in(0,1), E\left[\alpha^{*}\right]=1\end{array}$ & $\begin{array}{c}\text { Failed State A. } \\
\text { Roving Bandit outcome } \\
E\left(\bar{\lambda}_{1}^{1}\right)^{*}=E\left(\bar{\lambda}_{1}^{2}\right)^{*}=0 \\
E\left[\rho^{*}\right] \in(0,1), E\left[\alpha^{*}\right]=1\end{array}$ \\
\hline
\end{tabular}

The following is the core finding of this work. we use the above results to examine the basic question raised in the introduction: what are the effect of public perception on different policy 
dimensions on the likelihood of coups and revolts. In other words, we are interested in the relationship between public perception $\psi$ and equilibrium choices. Hence we will only consider the regions in the space $(\zeta, \phi)$ where players' behavior depends on $\psi$. Specifically, this region is

$$
(\zeta, \phi) \in\left[\frac{1}{X-\psi \Delta^{2}}, \frac{1}{X-\left(\Delta^{1}+\Delta^{2}\right)}\right] \times[0,1] \cup\left[0, \frac{1}{X-\psi \Delta^{2}}\right] \times\left[\frac{X}{\pi\left(\Delta^{1}+\Delta^{2}-\psi \Delta^{2}\right)+X}, 1\right] .
$$

consisting of the Autocratic and of the (Mixed) Semi Efficient Autocracy with and without credible revolution threat.

The results of this section are organized in a series of corollaries on the links between the degree of opacity on a policy dimension and the properties of autocratic polities.

COROllary 1. The probability of having a

1. congruent policy in first dimension is increasing in opacity $\psi$

2. congruent policy in second dimension is decreasing in opacity $\psi$.

Proof. From proposition A.1,

$$
\bar{\lambda}^{1}=\pi+(1-\pi)[(M) P E(\psi)+(M) F E(\psi)]=\pi+(1-\pi)[1-(M) F I(\psi)]
$$

moreover in the appendix which in turn implies

$$
\frac{\partial \bar{\lambda}^{1}}{\partial \psi}=-(1-\pi) \frac{\partial F I(\psi)}{\partial \psi}>0
$$

because of corollary A.1; similarly

$$
\bar{\lambda}^{2}=\pi+(1-\pi)[(M) F E(\psi)] \Rightarrow \frac{\partial \bar{\lambda}^{2}}{\partial \psi}=(1-\pi) \frac{\partial F E(\psi)}{\partial \psi}<0
$$

again because of corollary A.1.

The following figure represents the situation

Note that this result provides an explanation for observed heterogeneity on policy dimensions within an autocracy. ${ }^{16}$

Corollary 2. In a Semi Efficient Autocracy, the probability of a coup is non monotone in opacity $\psi$ and has an interior maximum.

\footnotetext{
${ }^{16} \mathrm{An}$ interesting case study on divergent policy choices connected to information control for the Francoist dictatorship is provided by Gago-Rodrìguez and Nùnez-Nickel 2017.
} 


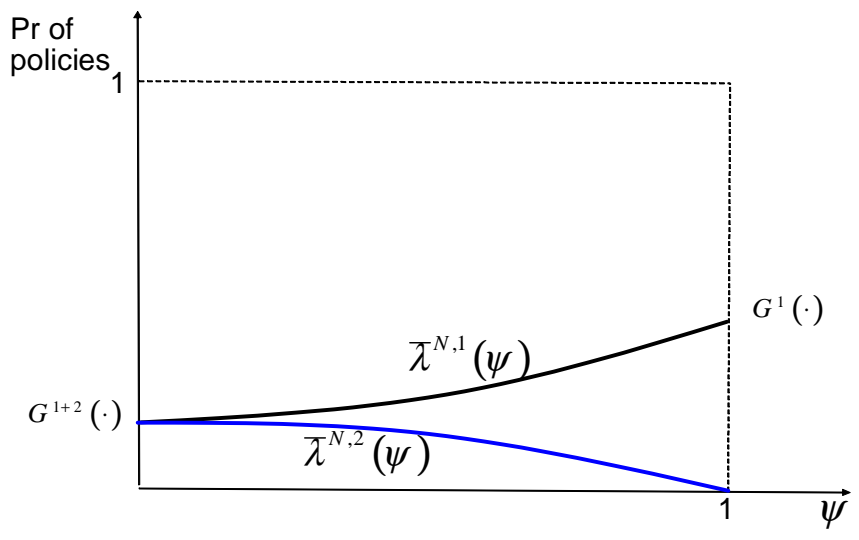

FIG. 2 Congruent policies on the two dimensions and opacity.

Proof. Since $E[\rho=1 \mid \psi]=F I(\psi)+P E(\psi)(1-\psi)$, then an increase in the opacity parameter has two opposite effects, a substitution effect because of the shift from full inefficiency $(F I(\psi) \downarrow)$ to partially efficiency $(P E(\psi) \uparrow)$, and a risk effect because the increment in partial efficient policy $(P E(\psi) \uparrow)$ increases the risk that the policy outcome reveals a non congruent dictator. Therefore

$$
\psi \uparrow \Rightarrow\left\{\begin{array}{lc}
E[\rho=1 \mid \psi] \downarrow & \text { because of substitution } \\
E[\rho=1 \mid \psi] \uparrow & \text { because of risk }
\end{array}\right.
$$

Formally

$$
\frac{\partial E[\rho=0 \mid \psi]}{\partial \psi}=\underbrace{\frac{\partial F I(\psi)}{\partial \psi}-P E(\psi)}_{\text {substitution } \text { effect }<0}+\underbrace{\frac{\partial P E(\psi)}{\partial \psi}(1-\psi)}_{\text {risk effect }>0} \gtrless 0:
$$

in particular as $\psi \rightarrow 0$ the risk effect is predominant, when $\psi \rightarrow 1$ the substitution effect prevails.

The following figure shows the situation

This result implies that exogenous changes in the autocratic control on public information might be dangerous from the ruler point of view because they might temporary increase the probability of a coup.

Finally, let we consider the probability of a revolt as a function of opacity. Of course, the only autocratic regime where this probability is not zero or one is the case of Mixed Semi Efficient Autocracies. 


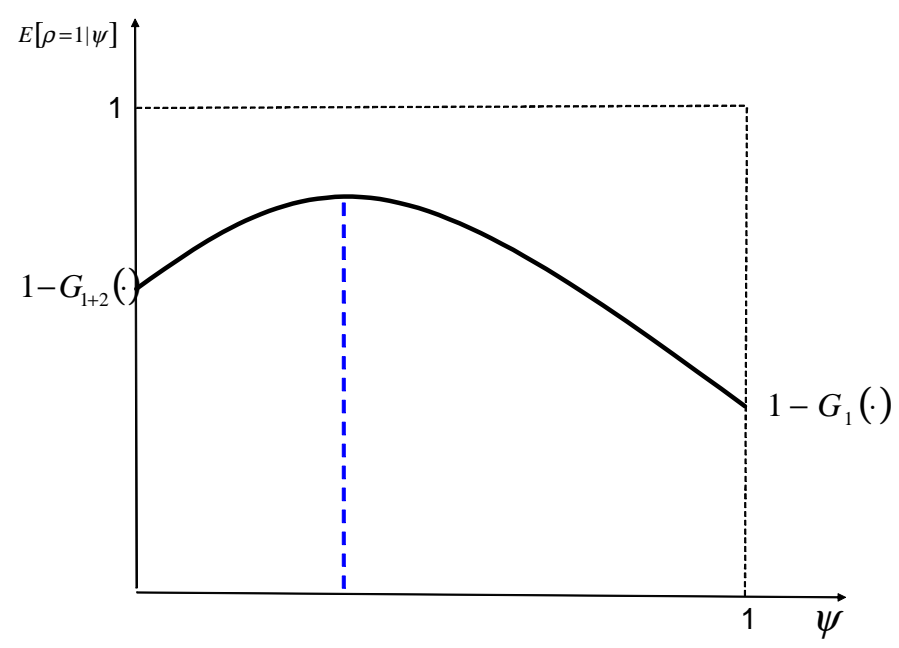

FIG. 3 Probability of a coup as a function of opacity.

Corollary 3. In a Mixed Semi Efficient Autocracy, the probability of a citizens' revolt might be non monotone in opacity $\psi$ and might have an interior minimum.

Proof. the probability of revolt in equilibrium is:

$$
\begin{gathered}
E(\alpha=1 \mid \psi)=1-E(\alpha=0 \mid \psi)=1-(1-\bar{\alpha})(M F E+\psi M P E)= \\
=1-(1-\bar{\alpha}) \frac{\pi}{1-\pi} \frac{1-\zeta\left(X-\left(\Delta^{1}+\Delta^{2}\right)\right)}{\zeta\left(X-\psi \Delta^{2}\right)-1}
\end{gathered}
$$

Thus

$$
\begin{aligned}
\frac{\partial E(\alpha=1 \mid \psi)}{\partial \psi}= & \frac{\pi}{1-\pi} \frac{1-\zeta\left(X-\left(\Delta^{1}+\Delta^{2}\right)\right)}{\zeta\left(X-\psi \Delta^{2}\right)-1} \frac{d \bar{\alpha}}{d \psi}-(1-\bar{\alpha}) \frac{\pi}{1-\pi} \frac{\zeta \Delta^{2}\left[1-\zeta\left(X-\left(\Delta^{1}+\Delta^{2}\right)\right)\right]}{\left[\zeta\left(X-\psi \Delta^{2}\right)-1\right]^{2}}= \\
& =\underbrace{\frac{\pi}{1-\pi} \frac{1-\zeta\left(X-\left(\Delta^{1}+\Delta^{2}\right)\right)}{\zeta\left(X-\psi \Delta^{2}\right)-1}}_{>0}[\underbrace{\frac{d \bar{\alpha}}{d \psi}}_{\lessgtr 0}-\underbrace{\frac{(1-\bar{\alpha}) \zeta \Delta^{2}}{\zeta\left(X-\psi \Delta^{2}\right)-1}}_{\geq 0}] \lessgtr 0
\end{aligned}
$$

Since $1-\bar{\alpha}(\psi)^{17}$ is decreasing, it is perfectly possible that

$$
\left.\frac{\partial E(\alpha=1 \mid \psi)}{\partial \psi}\right|_{\psi=0} \leq 0 \text { and }\left.\frac{\partial E(\alpha=1 \mid \psi)}{\partial \psi}\right|_{\psi=1} \geq 0
$$

so that the probability of having a revolt is minimized as opacity $\psi$ is intermediate.

\footnotetext{
${ }^{17}$ See remark 2 in the Appendix.
} 


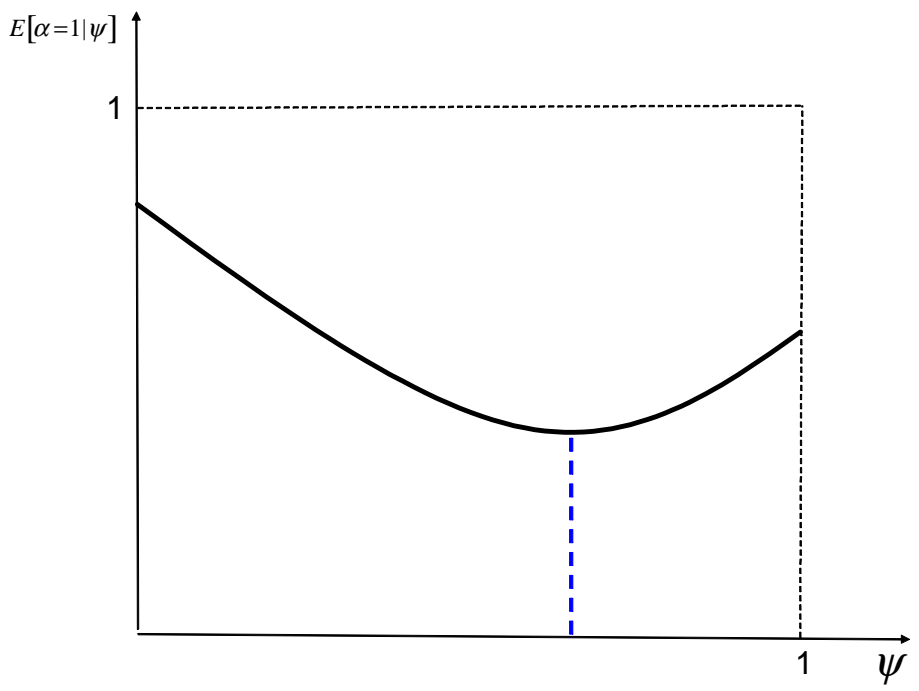

The reason for this result is related to the interaction between the probability of partially efficient policy and the public perception: as opacity increase, the probability of full inefficient and of full efficient policies decrease, so that the probability of a revolt might increase or decrease depending on the composition of this forces, however the probability of a partially efficient policy increases as well as the likelihood of observing an event that does not trigger a revolt with certainty; finally also the endogenous probability of a revolt is increasing with opacity. The net effect of the composition of these four different forces might well lead to the above non monotonic behavior. The following figure shows the possible situation.

The above results show that the effect of public perception of policy outcome on an authoritarian regime's stability is non monotone, in particular:

1. the probability of observing an efficient policy on some dimensions is decreasing in transparency, on other dimensions is increasing in public perception

2. the probability of a coup is not monotone in public perception

3. the probability of a revolt might be not monotone in public perception.

The first point provides an explanation of why the same autocratic leader implement different types of policies on different policy dimensions. The second and third points demonstrate the non-monotonic relationship between public perception and the probability of coups or revolts. The non-monotonicity is driven by the endogenously determined policy choices of the leaders. In particular, as opacity of public perception increases, the probability of partially efficient policy 
would increase, which in turn means an increase in the risk of actually observing a full inefficient outcome. This might increase the endogenous probability of a coup or revolt. These results are innovative, since usually it is assumed that a leader's control of public information has a positive monotonic effect on a regime's stability. Moreover, these results explain why the transition from a highly authoritarian dictatorship towards a more liberal one, or vice versa, is a very fragile process. This is because the probability of coups or revolts might increase. For these reasons, we think that our results are useful especially for transitional periods, when instability might arise suddenly because of unforeseen changes in public perception of policy dimensions.

\section{TWO CASE STUDIES}

In this section, we consider two case studies to show the potential of our results. Qualitative case studies might be useful in a situation where econometric studies because available data on regime changes, such as coups and revolts, have significant measurement problems, since the indicators differ widely and lead to unstable outcomes, as persuasively argued by Lueders and Lust 2017. Of course, the comparison of complicated periods of social changes in different countries is not without conceptual challenges and pitfalls, and these discussions cannot replace a controlled experiment with clear-cut causal inferences. But we are not intend to use case study as a test of our theory. We just want to illustrate the heuristic potential of our results.

Before discussing the specific cases, let we stress that our results should be applied with attention. In particular, it might seem counterfactual to assume that opacity is exogenous and not in full control of dictators. Our point is that most political change take place either at critical juncture of history, when it is plausible to assume that autocracies are not able to fully control the rapid changes in public perception or in situations of planned transition that however by definition should pass through changes and intermediate steps. In other words, we consider cases where there are either slow exogenous change towards more liberal flows of information or attempts of increasing autocratic control of public perception that have exogenous lags that affect the risk of coups and of revolts. Actually, our results might be also used to explain leaders' behavior with respect to the speed of attempted controls of the media as a way of affecting public perception and thus affecting the probability of coups or revolts, however this interpretation is outside the strict domain of this model. Of course, the complexity of revolutions and coups forces any analysis to highlight only specific dimensions of these phenomena. And this is of course even more true for a specific model as ours. However, even if from an historical point of view, real 
coups and real revolutions are incomparable, from an analytical point of view it might be useful to compare different cases to enlighten shared characteristics. Finally let we stress that we don't claim that coups or revolts are the inevitable outcome of changes in public perception of some policy dimensions, we simply point at a channel that affects the probability of an autocratic regime stability in a non monotonic way and that therefore might have unexpected consequences in transitional periods. We are well aware that other channels and processes affect the likelihood of coups and revolts in autocratic polities and we believe that their comprehension must involve the elaboration of more global models of how institutions, public beliefs, economics and politics are interconnected and, in particular, of how changes in one factor affects others.

\subsubsection{Soviet Union under Gorbachev ${ }^{18}$}

The first case study we consider is Soviet Union from 1985 till its dissolution in 1991, under the rule of Mikhail Gorbachev. Gorbachev was elected General Secretary by the Politburo on March 11, 1985. His initial goal as general secretary was to revive the Soviet economy that has been stagnating for many years. By 1982, the stagnation of the Soviet economy was clear to the political elite, but not to the main bulk of the Soviet citizens. The lack of products was covered by the importation from foreign countries, e.g. grain from US.

In general terms, Gorbachev chose political liberalization, while not implementing any significant economic reforms. By the time Gorbachev chose to characterizes his policies by his programs of glasnost (political openness and dramatic reduction in censorship), uskoreniye (speed-up of economic development) and perestroika (political and economic restructuring) announced in 1986 , the Soviet economy suffered from both hidden inflation and pervasive supply shortages aggravated by an increasingly open black market that undermined the official economy. Glasnost resulted in greater freedom of speech and the press becoming far less controlled. Thousands of political prisoners and many dissidents were also released. Soviet social science became free to explore and publish on many subjects that had previously been off limits, including conducting public opinion polls. The All-Union Center for Public Opinion Research (VCIOM) - the most prominent of several polling organizations that were started then - was opened. State archives became more accessible, and some social statistics that had been kept secret became open for research and publication on sensitive subjects such as income disparities, crime, suicide, abortion, and infant mortality. In terms of our model, the opacity on some policy dimensions was dramatically reduced.

\footnotetext{
${ }^{18}$ This case study is based on Kramer 2003 and Marples 2011.
} 
According to the implications of our model, Gorbachev's political and economic reforms had many significant unintended consequences. Relaxation of censorship under glasnost resulted in the Communist Party losing its absolute grip on the media. Before long, the media began to expose social and economic problems the Soviet government had long denied and actively concealed, such as poor housing, alcoholism, drug abuse, and pollution etc. Moreover, the ongoing war in Afghanistan, and the mishandling of the 1986 Chernobyl disaster, further damaged the credibility of the Soviet government at a time when dissatisfaction was increasing. Is it accidental that glasnost induced the emergence of a significant opposition to the Communist regimes in the Soviet Union and other eastern European countries from environmentally-oriented groups and citizens? The slogans of protection of the habitat, man and the nation were partly put forward because of the political conditions prevailing - where outright political protests would have been considered anti-Communists propaganda. However, they mainly reflected the deep concern of ordinary citizens to protect their own health and welfare once more information on the awful environmental conditions were widely disclosed. Public concern about the dangerous level of pollution and the degradation of nature triggered the development of nationalist and anti-government protests on a wide scale - particularly in the Baltic republics, Armenia and the Ukraine. In general, the positive view of Soviet life long presented to the public by the official media was rapidly fading, and the negative aspects of life in the Soviet Union were brought into the spotlight. Emboldened by the liberalized atmosphere of glasnost, public dissatisfaction with economic conditions was much more overt than ever before in the Soviet period.

On the other hand, although perestroika was considered bold in the context of Soviet history, Gorbachev's attempts at economic reform were not radical enough to restart the country's chronically sluggish economy. The reforms made some inroads in decentralization, but Gorbachev and his team left intact most of the fundamental elements of the Stalinist system, including price controls, inconvertibility of the ruble, exclusion of private property ownership, and the government monopoly over most means of production.

The political outcome of this combination of reduction in public opinion manipulation together with worsening economic conditions but successful foreign policy, were dramatic, but understandable using our results. According to corollary 1, when opacity is high, as it was before glasnost, the probability of full efficient policies is low. As enlightened by corollary 2, a reduction in opacity initially increases the likelihood of a coup. Faced with growing separatism, Gorbachev sought to restructure the Soviet Union into a less centralized state. On August 20, 1991, the Russian SFSR was scheduled to sign a New Union Treaty that would have converted 
the Soviet Union into a federation of independent republics with a common president, foreign policy and military. On August 19, 1991, Gorbachev's vice president, Gennady Yanayev, Prime Minister Valentin Pavlov, Defense Minister Dmitry Yazov, KGB chief Vladimir Kryuchkov and other senior officials acted to prevent the union treaty from being signed by forming the "General Committee on the State Emergency," which put Gorbachev - on holiday in Foros, Crimea - under house arrest and cut off his communications. The coup leaders issued an emergency decree suspending political activity and banning most newspapers. Coup organizers expected some popular support but found that public sympathy in large cities and in the republics was largely against them, manifested by public demonstrations, especially in Moscow. After three days, on August 21, 1991, the coup collapsed. The organizers were detained and Gorbachev returned as president, albeit with his power much depleted. In a nationally televised speech early in the morning of December 25, 1991, Gorbachev resigned as president of the USSR. On December 26, the upper chamber of the Union's Supreme Soviet voted both itself and the Soviet Union out of existence.

\subsubsection{Turkey 2013-2016: a new possible case? ${ }^{19}$}

Turkey and its recent political development represents a further interesting case study to analyze using the results and the intuitions provided by this work. First, let we stress that even if many scholars might consider Turkey as a democracy, however the great majority think that actually in the last five years Erdogan is abandoning democracy and it is now a partial autocracy masquerading as a democracy. Moreover, Turkey adapt quite well to our setting because in the last five to ten years it is characterized by a successful economic policy, with average GDP growth rate at 7.1 in the years from 2011 to $2015,{ }^{20}$ but also by a quite ineffective foreign policy. From this point of view, the increased power and authority of the ruling Justice and Development Party (AKP) and of its leader Recep Tayyip Erdogan generate a foreign policy that largely disregard the historical Turkish position of 'minimal engagement with the Middle East'. Since the early 2000s, successive Turkish governments have invested greatly in Middle East policy, in particular since 2011 the government's policy focused on removing Assad as dictator of Syria, however this policy did not lead to the expected outcomes. The rapid internationalization of the Syrian conflict, the ethnic and political ties between the Syrian Kurds and Turkey's own Kurdish population, and the emergence of the ISIS threat have accelerated the spread of the civil war

\footnotetext{
${ }^{19}$ This section is based on Yakıs, 2014, Gunter 2016, Yavuz and Balci 2018.

${ }^{20}$ Data of the World Bank.
} 
and of international terrorism activities across the Turkish border. The gradual normalization of ties with Russia since June 2016, and the changing geopolitical equations in the aftermath of the battle for Aleppo appear to have shifted Turkey's Syrian calculus, which is now more rooted in pragmatism than before. The Syrian war has also made relations with the EU more complex for Turkey. Caught out by the side effects of the Syrian civil war and deteriorating relations with the EU and the US, Turkey now appears to be in search of new alternatives to overcome its current limitations. The Turkish model, widely promoted by Western circles in the 2000s, particularly after the 2011 Arab revolts, has lost its sheen in the context of the Syrian civil war and Turkish foreign policy is now actually gridlock and widely seen as quite unsuccessful. ${ }^{21}$

Let now consider the internal political events in Turkey in the last five years. In the spring and summer of 2013, quite unexpectedly, a peaceful sit-in in Gezi Park in Istanbul escalated into a country-wise protest movement. Triggered by violent police crackdown and precipitated by Erdogan's defiant rhetoric, the demonstrations quickly spread to other cities (there had been more than 200 protests in 67 cities across the country). The protest start in May 27th as an environmental movement, a classic policy dimension that is not easily observable, however the excessive use of force by police in the subsequent days immediately spread to many citizens through social media. The government's reaction was one of bewilderment. After the initial shock, Erdogan took control of mostly classic media and tried to control the social media too, however the information spread across the country and the citizens. By June 23rd, according to the Ministry of Interior 2.5 million people have taken part in demonstration in 79 of Turkey's 81 provinces, and approximately 4.900 people had been detained because of such protests, 6 killed and more than 8.000 wounded. Apart from direct repression, the government answer was to restrict freedom of the classic and of social media. The protest movement was followed by a first crackdown on media with a shrinking space for reporting on issues the government does not want covered.

What was the outcome of this increase in the manipulation of public opinion? According to our results, we should expect a reduction in the probability of a citizens' revolt and an increase in the likelihood of a coup. And this is exactly what happened. The result of this control on public information, was that according to opinion poll, among the citizens the support for the government increased from $35.3 \%$ to $43 \%$. On the other hand, on the night of July 15, 2016, elements of the Turkish military staged a coup attempt that failed in few hours. According to official figures, at least 241 citizens and security personnel were killed, and over 2,000 more injured during

\footnotetext{
${ }^{21}$ Dalay, 2016.
} 
the clashes in the capital Ankara and Istanbul. On July 21, President Recep Tayyip Erdoğan declared a three-month state of emergency. His reaction on controlling public information was absolutely coherent with his aim of reducing the possibility of further coups. By December 2016, 140 media outlets and 29 publishing houses had been shut down via emergency decree, leaving more than 2,500 media workers and journalists unemployed. The government engineered the takeover of privately-owned media and other organizations by appointing government-approved trustees to run them. Arrest warrants have been issued for more than 100 journalists, and, according to P24, an independent journalism platform, 149 journalists and media workers now languish in Turkish jails making Turkey the world leader in locking up journalists. ${ }^{22}$

All these facts are perfectly coherent with the implications of our model, both the fact that policy dimensions have very different effectiveness (corollaries 4 and 5) and that a first increment in public opinion manipulation might lead to an increase in coup attempts, and finally that the most effective answer to such a threat is a further increment in censorship (corollary 6).

\section{CONCLUSION}

In this paper, we analyze the effect of public perception on different policy dimensions on regime stability in autocracies. We find that increasing control of public information might lead to an increase in coup and revolt attempts. The finding is consistent with the historical experience of some authoritarian regimes during transitional period.

Although our results have the potential to apply in a more generalized setting, it is important to keep two points in mind. First, the complexity of coups and revolutions allow researchers to touch only limited truth. Therefore, we do not intend to claim a specific model as ours provide the one and only version of all reality. Second, we do not claim coups and revolutions are the inevitable outcome of changes in public perception of some policy dimensions. We just want to illustrate a mechanism that can affect the stability of autocratic regime in a non monotonic way during the transitional periods. We are well aware that other mechanisms may also exist and, we believe that their comprehension must involve the elaboration of more global models of how institutions and public beliefs are interrelated.

\footnotetext{
${ }^{22}$ Human Rights Watch, 2016.
} 


\section{REFERENCES}

[1] Acemoglu, D., Robinson, J., and Verdier T., 2004. Kleptocracy and divide-and-rule: a model of personal rule. Journal of European Economic Association, 2(3), 162-192.

[2] Acemoglu, D., Robinson, J., 2006. Economic Origins of Dictatorship and Democracy. University Press, Cambridge.

[3] Acemoglu D., Egorov G., and Sonin K., 2010. Political selection and persistence of bad governments. The Quarterly Journal of Economics, 125(4), 1511-1575.

[4] Ades A., and Chua H. 1997. Thy neighbor's curse: regional instability and economic growth. Journal of Economic Growth, 2(3), 279-304.

[5] Aghion P., Alesina A., and Trebbi F., 2004. Endogenous political institutions. Quarterly Journal of Economics, 119, 565-611.

[6] Aidt T., and Jensen P., 2014. Workers of the world, unite! Franchise extensions and the threat of revolution in Europe, 1820-1938. European Economic Review, 72, 52-75.

[7] Alesina A., Ozler S., Roubini N., and Swagel P,. 1996. Political instability and economic growth. Journal of Economic Growth, 1(2), 189-211.

[8] Almeida H., and Ferreira D., 2002. Democracy and the variability of economic performance. Economics and Politics, 14(3), 225-257.

[9] Apolte, T., 2012. Why is there no revolution in North Korea. Public Choice, 150(2), 561-578.

[10] Maximilian Auffhammer M., Sun W., Wu J. and Zheng S. 2016. The Decomposition and Dynamics of Industrial Carbon Dioxide Emissions for 287 Chines Cities in 1998-2009. Journal of Economic Surveys (2016) Vol. 30, No. 3, pp. 460-481.

[11] Berganze J., 2000. Two roles for elections: disciplining the incumbent and selecting a competent candidate. Public Choice, 105,165-193.

[12] Besley T., 2006. Principled Agents? The Political Economy of Good Government. Oxford University Press.

[13] Besley, Timothy and Andrea Prat. 2006. Handcuffs for the Grabbing Hand? The Role of the Media in Political Accountability. American Economic Review, 96(3): 720-736. 
[14] Besley T. and Kudamatsu M., 2008. Making autocracy work. In: Helpman E. (Eds.), Institutions and Economic Performance, Harvard University Press, chapter 11, pp. 452-510.

[15] Blomberg, B. 1996. Growth, political instability and the defence burden. Economica 63, 649-672.

[16] Boix C. and Svolik M., 2013. The foundations of limited authoritarian government: institutions, commitment, and power-sharing in dictatorships. Journal of Politics, 75(2), 300-316.

[17] Brookings Instititution, 2008. Index of State Weakness in the Developing World. http://www.brookings.edu/research/reports/2008/02/weak-states-index.

[18] Bueno de Mesquita B., Smith J., Siverson R. and Morrow A., 2003. The Logic of Political Survival. The MIT press.

[19] Bueno de Mesquita B, and Smith A., 2009. Political Survival and Endogenous Institutional Change. Comparative Political Studies, Volume 42, Number 2, 167-197.

[20] Bueno de Mesquita B, and Smith A., 2010. Leader Survival, Revolutions, and the Nature of Government Finance. American Journal of Political Science 54(4), 936-950.

[21] Dalay, G. 2016. Turkey's Post-Arab Spring Foreign Policy. Foreign Policy, November.

[22] Darby, J., Li, C., and Muscatelli, V., 2004. Political uncertainty, public expenditure and growth. European Journal of Political Economy 20, 153-179.

[23] Dewatripont, M.; Jewitt, I. and Tirole, J., 1999. The Economics of Career Concerns, Part I: Comparing Information Structures. Review of Economic Studies, 66(1): 183-198.

[24] Egorov G., Guriev S. M. and Sonin, K., 2009. Why resource-Poor dictators allow freer media: a theory and evidence from panel data. American Political Science Review, 103, 4: 645-668.

[25] Egorov G. and Sonin, K., 2011. Dictators and their viziers: endogenizing loyalty-competence trade-off. Journal of the European Economic Association, 9, 5:903-930.

[26] Ferejohn, John. 1999. Accountability and Authority: Toward a Theory of Political Accountability, In Democracy, Accountability, and Representation, eds. Adam Przeworski, Susan C. Stokes, and Bernard Manin. Cambridge University Press, 131-153. 
[27] Friedrich C. and Brzezinski Z. (1965) Totalitarian Dictatorship and Autocracy. Cambridge: Harvard University Press

[28] Fund for Peace and Foreign Policy, 2012. Failed States Index, http://www.foreignpolicy.com/failedstates2012.

[29] Gago-Rodrìguez S and Nùnex-Nickel M 2017. Autocratic tensions, cronyism, and the opacity of business information: party newspapers and circulation figures during the Fancoist dictatorship (1939-1975). Business Ethics: a European Review, Vol. 26, N.1: 80-95.

[30] Gandhi J., 2008. Political Institutions Under Dictatorship. Cambridge University Press, Cambridge.

[31] Gandhi J. and Przeworski, A., 2006. Cooperation, cooptation, and rebellion under dictatorships. Economics and Politics, 18, 1-26.

[32] Gandhi J. and Przeworski, A., 2007. Authoritarian institutions and the survival of autocrats. Comparative Political Studies, 40, 11: 1279-1301.

[33] Galetovic A. and Sanhuenza R., 2000. Citizens, autocrats, and plotters: a model and new evidence on coups d'état. Economics and Politics, 12, 183-2044.

[34] Geddes. B., 1999. What do we know about democratization after twenty years? Annual Review of Political Science, 2: 115-144.

[35] Gehlbach S. and Keefer P., 2011. Investment without democracy: ruling-party institutionalization and credible commitment in autocracies. Journal of Comparative Economics, 39(2): 123-139.

[36] Gilli M. and Li Y., 2013. A model of Chinese central government: the role of reciprocal accountability. The Economics of Transition, 21(3), 451-477.

[37] Gilli M. and Li Y., 2014. Accountability in one-party government: rethinking the success of Chinese economic reform. Journal of Institutional and Theoretical Economics, 170 (4): 616-645.

[38] Gilli M. and Li Y., 2015. Coups, revolutions and efficient policies in autocracies. European Journal of Political Economy 39 (2015): 109-124. 
[39] Gilli M, Li Y. and Qian J. 2016. Logrolling Under Fragmented Authoritarianism: Theory and Evidence from China, University of Milan-Bicocca. Department of Economics, Management and Statistics Working Paper No. 333.

[40] Gilli M. and Li Y., 2017. A New Approach to Accountability in Autocracies. mimeo, University of Milan-Bicocca. Department of Economics, Management and Statistics.

[41] Gilli M. and Li Y., 2018. A note on Transparency and Incentives. mimeo, University of Milan-Bicocca. Department of Economics, Management and Statistics.

[42] Gunter M. 2016. Erdoğan and the Decline of Turkey. Middle East Policy, Volume 23, Issue 4, Pages 123-135

[43] Guriev S. and Treisman D. 2016. How Modern Dictator Survive: An Informational Theory of the New Authorianism. mimeo.

[44] Holmstrom, B., 1979 Moral Hazard and Observability. Bell Journal of Economics, 10: 74-91.

[45] Holmstrom, B., 1999. Managerial Incentive Problems: A Dynamic Perspective. Review of Economic Studies, 66(1): 169-182

[46] Human Rights Watch 2016. Silencing Turkey's Media, Report.

[47] Jones B. F. and Olken B., 2005. Do dictators matter? National dictatorship and growth since World War II. The Quarterly Journal of Economics, 120 (3), 835-864.

[48] Jong-A-Pin R. 2009. On the measurement of political instability and its impact on economic growth. European Journal of Political Economy 25, 15-29.

[49] Kramer M. 2003. Special Issue on "The Collapse of Soviet Union". Journal of Cold War Studies, Volume 5, Issue 4, p. 3-42.

[50] Li, Y., 2014. Downward accountability in response to collective actions: the political economy of public goods provision in China. The Economics of Transition, 22(1), p. 69-103.

[51] Linz, J. and Chehabi, H. E. eds. 1998. Sultanistic Regimes. Baltimore: Johns Hopkins University Press.

[52] Lipset, S. M., 1959. Some social requisites of democracy. American Political Science Review, $53,69-105$. 
[53] Londregan, J. and Poole, K. 1990. Poverty, the coup trap, and the seizure of executive power. World Politics 42(2),151-183.

[54] Lorentzen, P. 2014. China's Strategic Censorship. American Journal of Political Science, Vol. 58, No. 2: 402-414

[55] Lueders H. and Lust E. 2017. Multiple Measurements, Elusive Agreement, and Unstable Outcomes in the Study of Regime Changes. Working Paper 2017-52 V-Dem Institute, https://papers.ssrn.com/sol3/papers.cfm?abstract_id=3042470.

[56] Marples D. R. 2011. Revisiting the Collapse of the USSR. Canadian Slavonic Papers / Revue Canadienne des Slavistes, Vol. 53, No. 2/4, pp. 461-473

[57] Maskin E. and Tirole J., 2004. The politician and the judge: accountability in government. American Economic Review, 94, 4:1034-1054.

[58] McDaniel Tim 1191. Autocracy, Modernization, and Revolution in Russia and Iran. Princeton, Princeton University Press.

[59] Myerson, R. 2008. The autocrat's credibility problem and foundations of the constitutional state. The American Political Science Review, 102, 1: 125-139.

[60] O'Kane, R., 1981. A probabilistic approach to the causes of coups d'état. British Journal of Political Science, 11, 3: 287-308.

[61] Prat, A., 2005. The Wrong Kind of Transparency. The American Economic Review, Vol. 95, No. 3: 862-877

[62] Qin, Bei, David Strömberg and Yanhui Wu. 2014. Media Bias in China. Unpublished manuscript, http://www-bcf.usc.edu/ yanhuiwu/MediaBias1.pdf.

[63] Reinikka, Ritva, and Jakob Svensson 2005. Fighting Corruption to Improve Schooling: Evidence from a Newspaper Campaign in Uganda. Journal of the European Economic Association 3: 259-267.

[64] Rodrik, D., 1997. The 'paradoxes' of the successful state. European Economic Review, 41, 411-442.

[65] Straffin P.D., 1994. Power and stability in politics. In: Handbook of Game Theory, vol. 2, Elsevier, pp.1127-1151. 
[66] Smith A. 2008. The Perils of Unearned Income. The Journal of Politics, Vol. 70, No. 3, 780-793.

[67] Svolik, M., 2009. Power sharing and dictatorship dynamics in authoritarian regimes. American Journal of Political Science: 53, 2: 477-494.

[68] Svolik, M., 2012. The Politics of Authoritarian Rule. Cambridge University Press, Cambridge.

[69] Svolik, M., 2013. Contracting on violence: the moral hazard in authoritarian repression and military intervention in politics. Journal of Conflict Resolution, 57, 5: 765-794.

[70] Tullock, G., 1987. Autocracy. Kluwer Academic, Boston.

[71] Ulfelder, J., 2007, Natural-resource wealth and the survival of autocracy. Comparative Political Studies, 40, 8: 995-1018

[72] Van der Ploeg, F., 2007. Challenges and opportunities for resource rich economies. Journal of Economic Literature.

[73] Wright, J. 2008. Do authoritarian institutions constrain? How legislatures affect economic growth and investment. American Journal of Political Science, 52, 2: 322-343

[74] Yakış Y., 2014. Turkey after the Arab Spring: Policy Dilemmas. Middle East Policy, Volume 21, Issue 1, Pages 98-106

[75] Yavuz M. H. and Balci B. (eds) 2018. Turkey's July 15th Coup: What Happened and Why, The University of Utah Press 


\section{APPENDIX}

\section{THE PLAYERS' PAYOFFS}

The first period payoff of the noncongruent dictator $(N)$, of the selectorate $(S)$, of the congruent dictator $(C)$ and of the citizens $(Z)$, are respectively:

$$
\begin{aligned}
& U^{N}\left(e_{1} \mid \theta_{1}\right)=\left\{\begin{array}{ll}
\Delta^{1}+\Delta^{2}+\frac{X}{\phi} & \text { if } e_{1}^{1}=\theta_{1}^{1}, e_{1}^{2}=\theta_{1}^{2} \\
\Delta^{1}+\widetilde{\Delta}^{2}+r_{t}^{2}+\frac{X}{\phi} & \text { if } e_{1}^{1}=\theta_{1}^{1}, e_{1}^{2} \neq \theta_{1}^{2} \\
\Delta^{2}+r_{t}^{1}+\frac{X}{\phi} & \text { if } e_{1}^{1} \neq \theta_{1}^{1}, e_{1}^{2}=\theta_{1}^{2} \\
\widetilde{\Delta}^{2}+r_{t}^{1}+r_{t}^{2}+\frac{X}{\phi} & \text { if } e_{1}^{1} \neq \theta_{1}^{1}, e_{1}^{1} \neq \theta_{1}^{1}
\end{array} \quad \& \quad U^{C}\left(e_{1} \mid \theta_{1}\right)= \begin{cases}\Delta^{1}+\Delta^{2} & \text { if } e_{1}^{1}=\theta_{1}^{1}, e_{1}^{2}=\theta_{1}^{2} \\
\Delta^{1}+\widetilde{\Delta}^{2} & \text { if } e_{1}^{1}=\theta_{1}^{1}, e_{1}^{2} \neq \theta_{1}^{2} \\
\Delta^{2} & \text { if } e_{1}^{1} \neq \theta_{1}^{1}, e_{1}^{2}=\theta_{1}^{2} \\
\widetilde{\Delta}^{2} & \text { if } e_{1}^{1} \neq \theta_{1}^{1}, e_{1}^{1} \neq \theta_{1}^{1}\end{cases} \right. \\
& U^{S}\left(e_{1} \mid \theta_{1}\right)=\left\{\begin{array}{ll}
\Delta^{1}+\Delta^{2}+\frac{X}{\phi} & \text { if } e_{1}^{1}=\theta_{1}^{1}, e_{1}^{2}=\theta_{1}^{2} \\
\Delta^{1}+\widetilde{\Delta}^{2}+\frac{X}{\phi} & \text { if } e_{1}^{1}=\theta_{1}^{1}, e_{1}^{2} \neq \theta_{1}^{2} \\
\Delta^{2}+\frac{X}{\phi} & \text { if } e_{1}^{1} \neq \theta_{1}^{1}, e_{1}^{2}=\theta_{1}^{2} \\
\widetilde{\Delta}^{2}+\frac{X}{\phi} & \text { if } e_{1}^{1} \neq \theta_{1}^{1}, e_{1}^{1} \neq \theta_{1}^{1}
\end{array} \quad \& \quad U^{Z}\left(e_{1}\right)= \begin{cases}\Delta^{1}+\Delta^{2} & \text { if } e_{1}^{1}=\theta_{1}^{1}, e_{1}^{2}=\theta_{1}^{2} \\
\Delta^{1}+\widetilde{\Delta}^{2} & \text { if } e_{1}^{1}=\theta_{1}^{1}, e_{1}^{2} \neq \theta_{1}^{2} \\
\Delta^{2} & \text { if } e_{1}^{1} \neq \theta_{1}^{1}, e_{1}^{2}=\theta_{1}^{2} \\
\widetilde{\Delta}^{2} & \text { if } e_{1}^{1} \neq \theta_{1}^{1}, e_{1}^{1} \neq \theta_{1}^{1}\end{cases} \right.
\end{aligned}
$$

while in the second period they get ${ }^{23}$

$$
\begin{gathered}
U^{N}\left(e_{2} \mid \theta_{2}\right)= \begin{cases}\Delta^{1}+\Delta^{2}+\frac{X}{\phi} & \text { if } e_{2}^{1}=\theta_{2}^{1}, e_{2}^{2}=\theta_{2}^{2} \text { and no revolt nor coup } \\
\Delta^{1}+\widetilde{\Delta}^{2}+r_{t}^{2}+\frac{X}{\phi} & \text { if } e_{2}^{1}=\theta_{2}^{1}, e_{2}^{2} \neq \theta_{2}^{2} \text { and no revolt nor coup } \\
\Delta^{2}+r_{t}^{1}+\frac{X}{\phi} & \text { if } e_{2}^{1} \neq \theta_{2}^{1}, e_{2}^{2}=\theta_{2}^{2} \text { and no revolt nor coup } \\
\widetilde{\Delta}^{2}+r_{t}^{1}+r_{t}^{2}+\frac{X}{\phi} & \text { if } e_{2}^{1} \neq \theta_{2}^{1}, e_{2}^{2} \neq \theta_{2}^{2} \text { and no revolt nor coup } \\
0 & \text { if revolt or coup }\end{cases} \\
U^{C}\left(e_{2} \mid \theta_{2}\right)= \begin{cases}\Delta^{1}+\Delta^{2} & \text { if } e_{2}^{1}=\theta_{2}^{1}, e_{2}^{2}=\theta_{2}^{2} \text { and no revolt nor coup } \\
\Delta^{1}+\widetilde{\Delta}^{2} & \text { if } e_{2}^{1}=\theta_{2}^{1}, e_{2}^{2} \neq \theta_{2}^{2} \text { and no revolt nor coup } \\
\Delta^{2} & \text { if } e_{2}^{1} \neq \theta_{2}^{1}, e_{2}^{2}=\theta_{2}^{2} \text { and no revolt nor coup } \\
\widetilde{\Delta}^{2} & \text { if } e_{2}^{1} \neq \theta_{2}^{1}, e_{2}^{2} \neq \theta_{2}^{2} \text { and no revolt nor coup } \\
0 & \text { if revolt or coup }\end{cases}
\end{gathered}
$$

${ }^{23}$ The expected payoffs of the dictator and the selectorate when there is a revolt are normalized to 0. 


$$
\begin{aligned}
& U^{S}\left(e_{2} \mid \theta_{2}\right)=\left\{\begin{array}{lll}
\Delta^{1}+\Delta^{2}+\frac{X}{\phi} & \\
\Delta^{1}+\widetilde{\Delta}^{2}+\frac{X}{\phi} & \\
\Delta^{2}+\frac{X}{\phi} & \\
\widetilde{\Delta}^{2}+\frac{X}{\phi} & & \\
0 & & \\
& & \\
\Delta^{1}+\Delta^{2}+ & \frac{X}{\phi} & \text { with probability } \phi \\
& 0 & \text { with probability } 1-\phi \\
\Delta^{1}+\widetilde{\Delta}^{2}+\frac{X}{\phi} & \text { with probability } \phi \\
& 0 & \text { with probability } 1-\phi \\
\frac{X}{\phi} & \text { with probability } \phi \\
0 & \text { with probability } 1-\phi \\
\frac{X}{\phi} & \text { with probability } \phi \\
0 & \text { with probability } 1-\phi
\end{array}\right. \\
& U^{Z}\left(e_{2}\right)= \begin{cases}\Delta^{1}+\Delta^{2} \\
\Delta^{1}+\widetilde{\Delta}^{2} \\
\Delta^{2} \\
\widetilde{\Delta}^{2} \\
\frac{X-(\zeta)^{-1}}{1-\phi} & \text { with probability } 1-\phi \\
0 & \text { with probability } \phi\end{cases}
\end{aligned}
$$

if $e_{2}^{1}=\theta_{2}^{1}, e_{2}^{2}=\theta_{2}^{2}$ and no revolt nor coup if $e_{2}^{1}=\theta_{2}^{1}, e_{2}^{2} \neq \theta_{2}^{2}$ and no revolt nor coup if $e_{2}^{1} \neq \theta_{2}^{1}, e_{2}^{2}=\theta_{2}^{2}$ and no revolt nor coup if $e_{2}^{1} \neq \theta_{2}^{1}, e_{2}^{2} \neq \theta_{2}^{2}$ and no revolt nor coup if revolt

if $e_{2}^{1}=\theta_{2}^{1}, e_{2}^{2}=\theta_{2}^{2}$ and no revolt but coup if $e_{2}^{1}=\theta_{2}^{1}, e_{2}^{2} \neq \theta_{2}^{2}$ and no revolt but coup if $e_{2}^{1} \neq \theta_{2}^{1}, e_{2}^{2}=\theta_{2}^{2}$ and no revolt but coup if $e_{2}^{1} \neq \theta_{2}^{1}, e_{2}^{2} \neq \theta_{2}^{2}$ and no revolt but coup if $e_{2}^{1}=\theta_{2}^{1}, e_{2}^{2}=\theta_{2}^{2}$ and no revolt if $e_{2}^{1}=\theta_{2}^{1}, e_{2}^{2} \neq \theta_{2}^{2}$ and no revolt if $e_{2}^{1} \neq \theta_{2}^{1}, e_{2}^{2}=\theta_{2}^{2}$ and no revolt if $e_{2}^{1} \neq \theta_{2}^{1}, e_{2}^{2} \neq \theta_{2}^{2}$ and no revolt if revolt

Hence, in expected terms, in the first stage they get

$$
\begin{aligned}
& E\left[U^{N}\left(e_{1}, \theta_{1}\right)\right]=\left\{\begin{array}{ll}
\Delta^{1}+\Delta^{2}+\frac{X}{\phi} & \text { if } e_{1}^{1}=\theta_{1}^{1}, e_{1}^{2}=\theta_{1}^{2} \\
\Delta^{1}+\psi \Delta^{2}+r_{t}^{2}+\frac{X}{\phi} & \text { if } e_{1}^{1}=\theta_{1}^{1}, e_{1}^{2} \neq \theta_{1}^{2} \\
\Delta^{2}+r_{t}^{1}+\frac{X}{\phi} & \text { if } e_{1}^{1} \neq \theta_{1}^{1}, e_{1}^{2}=\theta_{1}^{2} \\
\psi \Delta^{2}+r_{t}^{1}+r_{t}^{2}+\frac{X}{\phi} & \text { if } e_{1}^{1} \neq \theta_{1}^{1}, e_{1}^{1} \neq \theta_{1}^{1}
\end{array} \quad \& E\left[U^{C}\left(e_{1}, \theta_{1}\right)\right]= \begin{cases}\Delta^{1}+\Delta^{2} & \text { if } e_{1}^{1}=\theta_{1}^{1}, e_{1}^{2}=\theta^{2} \\
\Delta^{1}+\psi \Delta^{2} & \text { if } e_{1}^{1}=\theta_{1}^{1}, e_{1}^{2} \neq \theta^{2} \\
\Delta^{2} & \text { if } e_{1}^{1} \neq \theta_{1}^{1}, e_{1}^{2}=\theta^{2} \\
\psi \Delta^{2} & \text { if } e_{1}^{1} \neq \theta_{1}^{1}, e_{1}^{1} \neq \theta^{2}\end{cases} \right. \\
& E\left[U^{S}\left(e_{1}, \theta_{1}\right)\right]=\left\{\begin{array}{ll}
\Delta^{1}+\Delta^{2}+\frac{X}{\phi} & \text { if } e_{1}^{1}=\theta_{1}^{1}, e_{1}^{2}=\theta_{1}^{2} \\
\Delta^{1}+\psi \Delta^{2}+\frac{X}{\phi} & \text { if } e_{1}^{1}=\theta_{1}^{1}, e_{1}^{2} \neq \theta_{1}^{2} \\
\Delta^{2}+\frac{X}{\phi} & \text { if } e_{1}^{1} \neq \theta_{1}^{1}, e_{1}^{2}=\theta_{1}^{2} \\
\psi \Delta^{2}+\frac{X}{\phi} & \text { if } e_{1}^{1} \neq \theta_{1}^{1}, e_{1}^{1} \neq \theta_{1}^{1}
\end{array} \& E\left[U^{Z}\left(e_{1}\right)\right]= \begin{cases}\Delta^{1}+\Delta^{2} & \text { if } e_{1}^{1}=\theta_{1}^{1}, e_{1}^{2}=\theta_{1}^{2} \\
\Delta^{1}+\psi \Delta^{2} & \text { if } e_{1}^{1}=\theta_{1}^{1}, e_{1}^{2} \neq \theta_{1}^{2} \\
\Delta^{2} & \text { if } e_{1}^{1} \neq \theta_{1}^{1}, e_{1}^{2}=\theta_{1}^{2} \\
\psi \Delta^{2} & \text { if } e_{1}^{1} \neq \theta_{1}^{1}, e_{1}^{1} \neq \theta_{1}^{1}\end{cases} \right.
\end{aligned}
$$




$$
E\left[U^{N}\left(e_{2} \mid \theta_{2}\right)\right]= \begin{cases}\Delta^{1}+\Delta^{2}+\frac{X}{\phi} & \text { if } e_{2}^{1}=\theta_{2}^{1}, e_{2}^{2}=\theta_{2}^{2} \text { and no revolt nor coup } \\ \Delta^{1}+\psi \Delta^{2}+r_{t}^{2}+\frac{X}{\phi} & \text { if } e_{2}^{1}=\theta_{2}^{1}, e_{2}^{2} \neq \theta_{2}^{2} \text { and no revolt nor coup } \\ \Delta^{2}+r_{t}^{1}+\frac{X}{\phi} & \text { if } e_{2}^{1} \neq \theta_{2}^{1}, e_{2}^{2}=\theta_{2}^{2} \text { and no revolt nor coup } \\ \psi \Delta^{2}+r_{t}^{1}+r_{t}^{2}+\frac{X}{\phi} & \text { if } e_{2}^{1} \neq \theta_{2}^{1}, e_{2}^{2} \neq \theta_{2}^{2} \text { and no revolt nor coup } \\ 0 & \text { if revolt or coup }\end{cases}
$$

$$
\begin{aligned}
& E\left[U^{C}\left(e_{2} \mid \theta_{2}\right)\right]= \begin{cases}\Delta^{1}+\Delta^{2} & \text { if } e_{2}^{1}=\theta_{2}^{1}, e_{2}^{2}=\theta_{2}^{2} \text { and no revolt nor coup } \\
\Delta^{1}+\psi \Delta^{2} & \text { if } e_{2}^{1}=\theta_{2}^{1}, e_{2}^{2} \neq \theta_{2}^{2} \text { and no revolt nor coup } \\
\Delta^{2} & \text { if } e_{2}^{1} \neq \theta_{2}^{1}, e_{2}^{2}=\theta_{2}^{2} \text { and no revolt nor coup } \\
\psi \Delta^{2} & \text { if } e_{2}^{1} \neq \theta_{2}^{1}, e_{2}^{2} \neq \theta_{2}^{2} \text { and no revolt nor coup } \\
0 & \text { if revolt or coup }\end{cases} \\
& E\left[U^{S}\left(e_{2} \mid \theta_{2}\right)\right]= \begin{cases}\Delta^{1}+\Delta^{2}+\frac{X}{\phi} & \text { if } e_{2}^{1}=\theta_{2}^{1}, e_{2}^{2}=\theta_{2}^{2} \text { and no revolt nor coup } \\
\Delta^{1}+\psi \Delta^{2}+\frac{X}{\phi} & \text { if } e_{2}^{1}=\theta_{2}^{1}, e_{2}^{2} \neq \theta_{2}^{2} \text { and no revolt nor coup } \\
\Delta^{2}+\frac{X}{\phi} & \text { if } e_{2}^{1} \neq \theta_{2}^{1}, e_{2}^{2}=\theta_{2}^{2} \text { and no revolt nor coup } \\
\psi \Delta^{2}+\frac{X}{\phi} & \text { if } e_{2}^{1} \neq \theta_{2}^{1}, e_{2}^{2} \neq \theta_{2}^{2} \text { and no revolt nor coup } \\
0 & \text { if revolt } \\
\Delta^{1}+\Delta^{2}+X & \text { if } e_{2}^{1}=\theta_{2}^{1}, e_{2}^{2}=\theta_{2}^{2} \text { and no revolt but coup } \\
\Delta^{1}+\psi \Delta^{2}+X & \text { if } e_{2}^{1}=\theta_{2}^{1}, e_{2}^{2} \neq \theta_{2}^{2} \text { and no revolt but coup } \\
\Delta^{2}+X & \text { if } e_{2}^{1} \neq \theta_{2}^{1}, e_{2}^{2}=\theta_{2}^{2} \text { and no revolt but coup } \\
\psi \Delta^{2}+X & \text { if } e_{2}^{1} \neq \theta_{2}^{1}, e_{2}^{2} \neq \theta_{2}^{2} \text { and no revolt but coup }\end{cases} \\
& E\left[U^{Z}\left(e_{2}\right)\right]= \begin{cases}\Delta^{1}+\Delta^{2} & \text { if } e_{2}^{1}=\theta_{2}^{1}, e_{2}^{2}=\theta_{2}^{2} \text { and no revolt } \\
\Delta^{1}+\psi \Delta^{2} & \text { if } e_{2}^{1}=\theta_{2}^{1}, e_{2}^{2} \neq \theta_{2}^{2} \text { and no revolt } \\
\Delta^{2} & \text { if } e_{2}^{1} \neq \theta_{2}^{1}, e_{2}^{2}=\theta_{2}^{2} \text { and no revolt } \\
\psi \Delta^{2} & \text { if } e_{2}^{1} \neq \theta_{2}^{1}, e_{2}^{2} \neq \theta_{2}^{2} \text { and no revolt } \\
X-(\zeta)^{-1} & \text { if revolt }\end{cases}
\end{aligned}
$$

Finally, let define the players' intertemporal payoff as follows

$$
W^{i}=E\left[U_{1}^{i}\right]+E\left[U_{2}^{i}\right], \quad i \in\{S, C, N, Z\} .
$$

\section{CALCULATIONS}

We use Sequential equilibrium (SE) as the solution concept instead of the more commonly used notion of Perfect Bayesian Equilibrium, since we have to analyze a three-player game and Sequential Equilibria encompass the notion of consistency which implies that players' beliefs 
about the true type of the leader agree out of the equilibrium path. Moreover, to refine out of equilibrium beliefs we use a strong version of Forward Induction in the sense that any deviations from full efficiency is interpreted as due to the non congruent leader. As usual, we work backwards to calculate the set of Sequential Equilibria. As explained in the main text, in these principal agents models, the second period choices are trivially given by their myopic best reply, exactly because it is the last period. Hence, we will analyze the players' behavior in the first-stage game, assuming that the players will play their best responses in the second final period.

\subsection{Sequential Rationality of the Players}

For each first stage leader's strategy profile, we analyze the citizens', the selectorate's and the leader's sequential rational behavior in each information set. The citizens' and the selectorate's sequential rational behavior depend on their posterior beliefs, derived by Bayes' rule from the leader's choices. Note that, by consistency, $\rho(\delta) \in(0,1)$; hence

$$
\mu^{Z}(C \mid \delta, \rho)=\mu^{S}(C \mid \delta)=: \mu(C \mid \delta) ;
$$

while if $\rho(\delta)=1$, there is a new appointed leader and thus, for any $\delta \in\{0, \Delta\}$

$$
\mu(C \mid \delta, \rho=1)=\pi
$$

Hence

$$
\mu(C \mid \delta, \rho=0)= \begin{cases}\frac{\pi\left[\bar{\lambda}^{C, 1} \bar{\lambda}^{C, 2}+\bar{\lambda}^{C, 1} \psi\left(1-\bar{\lambda}^{C, 2}\right)\right]}{\pi\left[\bar{\lambda}^{C, 1} \bar{\lambda}^{C, 2}+\lambda \psi\left(1-\bar{\lambda}^{C, 2}\right)\right]+(1-\pi)\left[\bar{\lambda}^{N, 1} \bar{\lambda}^{N, 2}+\bar{\lambda}^{N, 1} \psi\left(1-\bar{\lambda}^{N, 2}\right)\right]} & \delta=\Delta^{1}+\Delta^{2} \\ \frac{\pi\left[\left(1-\bar{\lambda}^{C, 1}\right) \bar{\lambda}^{C, 2}+\left(1-\bar{\lambda}^{C, 1}\right) \psi\left(1-\bar{\lambda}^{C, 2}\right)\right]}{\pi\left[\left(1-\bar{\lambda}^{C, 1}\right) \bar{\lambda}^{C, 2}+\left(1-\bar{\lambda}^{C, 1}\right) \psi\left(1-\bar{\lambda}^{C, 2}\right)\right]+(1-\pi)\left[\left(1-\bar{\lambda}^{N, 1}\right) \bar{\lambda}^{N, 2}+\left(1-\bar{\lambda}^{N, 1}\right) \psi\left(1-\bar{\lambda}^{N, 2}\right)\right]} & \delta=\Delta^{2} \\ \frac{\pi\left[\bar{\lambda}^{C, 1}(1-\psi)\left(1-\bar{\lambda}^{C, 2}\right)\right]}{\pi\left[\bar{\lambda}^{C, 1}(1-\psi)\left(1-\bar{\lambda}^{C, 2}\right)\right]+(1-\pi)\left[\bar{\lambda}^{N, 1}(1-\psi)\left(1-\bar{\lambda}^{N, 2}\right)\right]} & \delta=\Delta^{1} \\ \frac{\pi\left[\left(1-\bar{\lambda}^{C, 1}\right)(1-\psi)\left(1-\bar{\lambda}^{C, 2}\right)\right]}{\pi\left[\left(1-\bar{\lambda}^{C, 1}\right)(1-\psi)\left(1-\bar{\lambda}^{C, 2}\right)\right]+(1-\pi)\left[\left(1-\bar{\lambda}^{N, 1}\right)(1-\psi)\left(1-\bar{\lambda}^{N, 2}\right)\right]} & \delta=0\end{cases}
$$

Thus to calculate players' beliefs we should examine all the possible 16 dictator's first stage strategy profiles, however types $\mathrm{C}$ first stage strategies different from $\left(\bar{\lambda}^{C, 1}, \bar{\lambda}^{C, 2}\right)=(1,1)$ are weakly dominated and can be eliminated by any standard refinement of SE. Hence, we have to consider four first stage strategy profiles, holding $\left(\bar{\lambda}^{C, 1}, \bar{\lambda}^{C, 2}\right)=(1,1)$ fixed. Moreover, as stated before, to solve the cases of beliefs' indeterminacy out-of-the equilibrium path we consider a classic forward induction (FI) argument assuming that any deviation towards efficiency is due to the $\mathrm{C}$ type. 
1. Strategy profile $\left(\bar{\lambda}^{C, 1}, \bar{\lambda}^{C, 2}, \bar{\lambda}^{N, 1}, \bar{\lambda}^{N, 2}\right)=(1,1,0,0)$

The Citizens After knowing their first-period utility and the selectorate's choice at the end of the first period, the citizens choose between revolt $(\alpha=1)$ or not $(\alpha=0)$. This means that to derive the citizens' sequential rational behavior, we should consider eight possible information sets, where in each information set, there are two decision nodes depending on the type of leader. Let $V^{Z}(\alpha \mid \delta, \rho)$ be the expected continuation payoff for the citizens when they choose $\alpha$ if $(\delta, \rho)$ has been observed. The expected continuation utility that the citizens will get after they choose to initiate a revolution in $(\delta, \rho)$ is:

$$
V^{Z}(\alpha=1 \mid \delta, \rho)=(1-\phi) \times \frac{X-(\zeta)^{-1}}{1-\phi}+\phi \times 0=X-(\zeta)^{-1}
$$

Clearly, this payoff does not depend on their beliefs about the leader's type and thus on $(\delta, \rho)$. On the other hand, if the citizens decide to accommodate, the continuation payoff will depend on their beliefs about the type of leader which, in turn, will depend on their information at the time of deciding. Therefore, to find the citizens' rational behavior, we need to consider eight possible information sets, where $\widehat{\cdot}$ denotes the realization of the random variable $\cdot$

1. Information set $\left(\widehat{\delta}=\Delta^{1}+\Delta^{2}, \widehat{\rho}=0\right)$ In this information set the players' beliefs are $\mu\left(C \mid \widehat{\delta}=\Delta^{1}+\Delta^{2}\right)=\frac{\pi\left[\bar{\lambda}^{C, 1} \bar{\lambda}^{C, 2}+\bar{\lambda}^{C, 1} \psi\left(1-\bar{\lambda}^{C, 2}\right)\right]}{\pi\left[\bar{\lambda}^{C, 1} \bar{\lambda}^{C, 2}+\lambda \psi\left(1-\bar{\lambda}^{C, 2}\right)\right]+(1-\pi)\left[\bar{\lambda}^{N, 1} \bar{\lambda}^{N, 2}+\bar{\lambda}^{N, 1} \psi\left(1-\bar{\lambda}^{N, 2}\right)\right]}=1$

The expected continuation payoff the citizens will get after they choose not to revolt is $V^{Z}\left(\alpha=0 \mid \widehat{\delta}=\Delta^{1}+\Delta^{2}, \widehat{\rho}=0\right)=\mu\left(C \mid \widehat{\delta}=\Delta^{1}+\Delta^{2}\right)\left(\Delta^{1}+\Delta^{2}\right)+\left[1-\mu\left(C \mid \widehat{\delta}=\Delta^{1}+\Delta^{2}\right)\right] \psi \Delta^{2}=\Delta^{1}+\Delta^{2}$ then sequential rationality implies that the citizens will choose to revolt in $\left(\widehat{\delta}=\Delta^{1}+\Delta^{2}, \widehat{\rho}=0\right)$ if and only if

$$
\begin{gathered}
V^{Z}\left(\alpha=1 \mid \widehat{\delta}=\Delta^{1}+\Delta^{2}, \widehat{\rho}=0\right) \geq V^{Z}\left(\alpha=0 \mid \widehat{\delta}=\Delta^{1}+\Delta^{2}, \widehat{\rho}=0\right) \Leftrightarrow \\
\Leftrightarrow \alpha\left(\widehat{\delta}=\Delta^{1}+\Delta^{2}, \widehat{\rho}=0\right)=1 \Leftrightarrow X-(\zeta)^{-1} \geq \Delta^{1}+\Delta^{2} \Leftrightarrow(\zeta)^{-1} \leq X-\left(\Delta^{1}+\Delta^{2}\right) .
\end{gathered}
$$

2. Information set $\left(\widehat{\delta}=\Delta^{1}+\Delta^{2}, \widehat{\rho}=1\right)$ In this information set, the incumbent leader is removed from office by the selectorate; therefore, there is a new leader and thus the expected utility the citizens will get after they choose not to revolt does not depend on the previous 
observation on $\delta$. Then, the expected utility the citizens will get after they choose not to revolt is:

$$
V^{Z}\left(\alpha=0 \mid \widehat{\delta}=\Delta^{1}+\Delta^{2}, \widehat{\rho}=1\right)=\pi\left(\Delta^{1}+\Delta^{2}\right)+(1-\pi) \psi \Delta^{2} .
$$

Sequential rationality implies that the citizens will choose to revolt in $\left(\widehat{\delta}=\Delta^{1}+\Delta^{2}, \widehat{\rho}=0\right)$ if and only if

$$
\begin{gathered}
V^{Z}\left(\alpha=1 \mid \widehat{\delta}=\Delta^{1}+\Delta^{2}, \widehat{\rho}=1\right) \geq V^{Z}\left(\alpha=0 \mid \widehat{\delta}=\Delta^{1}+\Delta^{2}, \widehat{\rho}=1\right) \Leftrightarrow \\
\Leftrightarrow \alpha\left(\widehat{\delta}=\Delta^{1}+\Delta^{2}, \widehat{\rho}=1\right)=1 \Leftrightarrow(\zeta)^{-1} \leq X-\left[\pi\left(\Delta^{1}+\Delta^{2}\right)+(1-\pi) \psi \Delta^{2}\right] .
\end{gathered}
$$

3. Information set $\left(\widehat{\delta}=\Delta^{1}, \widehat{\rho}=0\right)$ In this information set the players' beliefs are

$$
\mu\left(C \mid \widehat{\delta}=\Delta^{1}\right)=\frac{\pi\left[\bar{\lambda}^{C, 1}(1-\psi)\left(1-\bar{\lambda}^{C, 2}\right)\right]}{\pi\left[\bar{\lambda}^{C, 1}(1-\psi)\left(1-\bar{\lambda}^{C, 2}\right)\right]+(1-\pi)\left[\bar{\lambda}^{N, 1}(1-\psi)\left(1-\bar{\lambda}^{N, 2}\right)\right]} \in[0,1] .
$$

Then, using FI we get

$$
\mu\left(C \mid \widehat{\delta}=\Delta^{1}\right)=0
$$

and the expected continuation payoff the citizens will get after they choose not to revolt is

$$
V^{Z}\left(\alpha=0 \mid \widehat{\delta}=\Delta^{1}, \widehat{\rho}=0\right)=\mu\left(C \mid \widehat{\delta}=\Delta^{1}\right)\left(\Delta^{1}+\Delta^{2}\right)+\left[1-\mu\left(C \mid \widehat{\delta}=\Delta^{1}\right)\right] \psi \Delta^{2}=\psi \Delta^{2}
$$

Thus sequential rationality implies that the citizens will choose to revolt in $\left(\widehat{\delta}=\Delta^{1}, \widehat{\rho}=0\right)$ if and only if

$$
\begin{gathered}
V^{Z}\left(\alpha=1 \mid \widehat{\delta}=\Delta^{1}, \widehat{\rho}=0\right) \geq V^{Z}\left(\alpha=0 \mid \widehat{\delta}=\Delta^{1}, \widehat{\rho}=0\right) \Leftrightarrow \\
\Leftrightarrow \alpha\left(\widehat{\delta}=\Delta^{1}, \widehat{\rho}=0\right)=1 \Leftrightarrow(\zeta)^{-1} \leq X-\psi \Delta^{2}
\end{gathered}
$$

4. Information set $\left(\widehat{\delta}=\Delta^{1}, \widehat{\rho}=1\right)$ In this information set, the incumbent leader is removed from office by the selectorate; therefore, there is a new leader and thus the expected utility the citizens will get after they choose not to revolt does not depend on the previous observation on $\delta$. Then, the expected utility the citizens will get after they choose not to revolt is:

$$
V^{Z}\left(\alpha=0 \mid \widehat{\delta}=\Delta^{1}, \widehat{\rho}=0\right)=\pi\left(\Delta^{1}+\Delta^{2}\right)+(1-\pi) \psi \Delta^{2} .
$$

Sequential rationality implies that the citizens will choose to revolt in $\left(\widehat{\delta}=\Delta^{1}, \widehat{\rho}=0\right)$ if and only if

$$
\begin{gathered}
V^{Z}\left(\alpha=1 \mid \widehat{\delta}=\Delta^{1}, \widehat{\rho}=0\right) \geq V^{Z}\left(\alpha=0 \mid \widehat{\delta}=\Delta^{1}, \widehat{\rho}=0\right) \Leftrightarrow \\
\Leftrightarrow \alpha\left(\widehat{\delta}=\Delta^{1}, \widehat{\rho}=0\right)=1 \Leftrightarrow(\zeta)^{-1} \leq X-\left[\pi\left(\Delta^{1}+\Delta^{2}\right)+(1-\pi) \psi \Delta^{2}\right] .
\end{gathered}
$$


5. Information set $\left(\widehat{\delta}=\Delta^{2}, \widehat{\rho}=0\right)$ In this information set the players' beliefs are

$$
\begin{gathered}
\mu\left(C \mid \widehat{\delta}=\Delta^{2}\right)= \\
=\frac{\pi\left[\left(1-\bar{\lambda}^{C, 1}\right) \bar{\lambda}^{C, 2}+\left(1-\bar{\lambda}^{C, 1}\right) \psi\left(1-\bar{\lambda}^{C, 2}\right)\right]}{\pi\left[\left(1-\bar{\lambda}^{C, 1}\right) \bar{\lambda}^{C, 2}+\left(1-\bar{\lambda}^{C, 1}\right) \psi\left(1-\bar{\lambda}^{C, 2}\right)\right]+(1-\pi)\left[\left(1-\bar{\lambda}^{N, 1}\right) \bar{\lambda}^{N, 2}+\left(1-\bar{\lambda}^{N, 1}\right) \psi\left(1-\bar{\lambda}^{N, 2}\right)\right]}=0 .
\end{gathered}
$$

Then the expected continuation payoff the citizens will get after they choose not to revolt is

$$
V^{Z}\left(\alpha=0 \mid \widehat{\delta}=\Delta^{2}, \widehat{\rho}=0\right)=\mu\left(C \mid \widehat{\delta}=\Delta^{2}\right)\left(\Delta^{1}+\Delta^{2}\right)+\left[1-\mu\left(C \mid \widehat{\delta}=\Delta^{2}\right)\right] \psi \Delta^{2}=0 .
$$

Thus sequential rationality implies that the citizens will choose to revolt in $\left(\widehat{\delta}=\Delta^{2}, \widehat{\rho}=0\right)$ if and only if

$$
\begin{gathered}
V^{Z}\left(\alpha=1 \mid \widehat{\delta}=\Delta^{2}, \widehat{\rho}=0\right) \geq V^{Z}\left(\alpha=0 \mid \widehat{\delta}=\Delta^{2}, \widehat{\rho}=0\right) \Leftrightarrow \\
\Leftrightarrow \alpha\left(\widehat{\delta}=\Delta^{2}, \widehat{\rho}=0\right)=1 \Leftrightarrow X-(\zeta)^{-1} \geq \psi \Delta^{2} \Leftrightarrow(\zeta)^{-1} \leq X-\psi \Delta^{2} .
\end{gathered}
$$

6. Information set $\left(\widehat{\delta}=\Delta^{2}, \widehat{\rho}=1\right)$ In this information set, the incumbent leader is removed from office by the selectorate; therefore, there is a new leader and thus the expected utility the citizens will get after they choose not to revolt does not depend on the previous observation on $\delta$. Then, the expected utility the citizens will get after they choose not to revolt is:

$$
V^{Z}\left(\alpha=0 \mid \widehat{\delta}=\Delta^{2}, \widehat{\rho}=1\right)=\pi\left(\Delta^{1}+\Delta^{2}\right)+(1-\pi) \psi \Delta^{2} .
$$

Sequential rationality implies that the citizens will choose to revolt in $\left(\widehat{\delta}=\Delta^{1}, \widehat{\rho}=1\right)$ if and only if

$$
\begin{gathered}
V^{Z}\left(\alpha=0 \mid \widehat{\delta}=\Delta^{2}, \widehat{\rho}=1\right) \geq V^{Z}\left(\alpha=0 \mid \widehat{\delta}=\Delta^{2}, \widehat{\rho}=1\right) \Leftrightarrow \\
\Leftrightarrow \alpha\left(\widehat{\delta}=\Delta^{2}, \widehat{\rho}=1\right)=1 \Leftrightarrow(\zeta)^{-1} \leq X-\left[\pi\left(\Delta^{1}+\Delta^{2}\right)+(1-\pi) \psi \Delta^{2}\right]
\end{gathered}
$$

7. Information set $(\widehat{\delta}=0, \widehat{\rho}=0)$ In this information set the players' beliefs are $\mu(C \mid \widehat{\delta}=0)=\frac{\pi\left[\left(1-\bar{\lambda}^{C, 1}\right)(1-\psi)\left(1-\bar{\lambda}^{C, 2}\right)\right]}{\pi\left[\left(1-\bar{\lambda}^{C, 1}\right)(1-\psi)\left(1-\bar{\lambda}^{C, 2}\right)\right]+(1-\pi)\left[\left(1-\bar{\lambda}^{N, 1}\right)(1-\psi)\left(1-\bar{\lambda}^{N, 2}\right)\right]}=0$.

Then the expected continuation payoff the citizens will get after they choose not to revolt is

$$
V^{Z}\left(\alpha=0 \mid \widehat{\delta}=\Delta^{2}, \widehat{\rho}=0\right)=\mu(C \mid \widehat{\delta}=0)\left(\Delta^{1}+\Delta^{2}\right)+[1-\mu(C \mid \widehat{\delta}=0)] \psi \Delta^{2}=\psi \Delta^{2}
$$


Thus sequential rationality implies that the citizens will choose to revolt in $\left(\widehat{\delta}=\Delta^{2}, \widehat{\rho}=0\right)$ if and only if

$$
\begin{gathered}
V^{Z}(\alpha=1 \mid \widehat{\delta}=0, \widehat{\rho}=0) \geq V^{Z}(\alpha=0 \mid \widehat{\delta}=0, \widehat{\rho}=0) \Leftrightarrow \\
\Leftrightarrow \alpha(\widehat{\delta}=0, \widehat{\rho}=1)=1 \Leftrightarrow X-(\zeta)^{-1} \geq \psi \Delta^{2} \Leftrightarrow(\zeta)^{-1} \leq X-\psi \Delta^{2} .
\end{gathered}
$$

8. Information set $(\widehat{\delta}=0, \widehat{\rho}=1)$ In this information set, the incumbent leader is removed from office by the selectorate; therefore, there is a new leader and thus the expected utility the citizens will get after they choose not to revolt does not depend on the previous observation on $\delta$. Then, the expected utility the citizens will get after they choose not to revolt is:

$$
V^{Z}(\alpha=0 \mid \widehat{\delta}=0, \widehat{\rho}=1)=\pi\left(\Delta^{1}+\Delta^{2}\right)+(1-\pi) \psi \Delta^{2} .
$$

Sequential rationality implies that the citizens will choose to revolt in $\left(\widehat{\delta}=\Delta^{1}, \widehat{\rho}=1\right)$ if and only if

$$
\begin{gathered}
V^{Z}(\alpha=0 \mid \widehat{\delta}=0, \widehat{\rho}=1) \geq V^{Z}(\alpha=0 \mid \widehat{\delta}=0, \widehat{\rho}=1) \Leftrightarrow \\
\Leftrightarrow \alpha(\widehat{\delta}=0, \widehat{\rho}=1)=1 \Leftrightarrow(\zeta)^{-1} \leq X-\left[\pi\left(\Delta^{1}+\Delta^{2}\right)+(1-\pi) \psi \Delta^{2}\right] .
\end{gathered}
$$

This analysis allows us to derive the following sequential best reply correspondences for each citizens' information set:

$$
\alpha(\widehat{\delta}, \widehat{\rho})^{B R}=\left\{\begin{array}{ccc}
1 \Leftrightarrow \zeta \in\left[\frac{1}{X-\left(\Delta^{1}+\Delta^{2}\right)}, 1\right] & \text { if } \quad\left(\widehat{\delta}=\Delta^{1}+\Delta^{2}, \widehat{\rho}=0\right) \\
1 \Leftrightarrow \zeta \in\left[\frac{1}{X-\left[\pi\left(\Delta^{1}+\Delta^{2}\right)+(1-\pi) \psi \Delta^{2}\right]}, 1\right] & \text { if } \quad\left(\widehat{\delta}=\Delta^{1}+\Delta^{2}, \widehat{\rho}=1\right) \\
1 \Leftrightarrow \zeta \in\left[\frac{1}{X-\psi \Delta^{2}}, 1\right] & \text { if } \quad\left(\widehat{\delta}=\Delta^{1}, \widehat{\rho}=0\right) \\
1 \Leftrightarrow \zeta \in\left[\frac{1}{X-\left[\pi\left(\Delta^{1}+\Delta^{2}\right)+(1-\pi) \psi \Delta^{2}\right]}, 1\right] & \text { if } \quad\left(\widehat{\delta}=\Delta^{1}, \widehat{\rho}=1\right) \\
1 \Leftrightarrow \zeta \in\left[\frac{1}{X-\psi \Delta^{2}}, 1\right] & \text { if } \quad\left(\widehat{\delta}=\Delta^{2}, \widehat{\rho}=0\right) \\
1 \Leftrightarrow \zeta \in\left[\frac{1}{X-\left[\pi\left(\Delta^{1}+\Delta^{2}\right)+(1-\pi) \psi \Delta^{2}\right]}, 1\right] & \text { if } \quad\left(\widehat{\delta}=\Delta^{2}, \widehat{\rho}=1\right) \\
1 \Leftrightarrow \zeta \in\left[\frac{1}{X-\psi \Delta^{2}}, 1\right] & \text { if } \quad(\widehat{\delta}=0, \widehat{\rho}=0) \\
1 \Leftrightarrow \zeta \in\left[\frac{1}{X-\left[\pi\left(\Delta^{1}+\Delta^{2}\right)+(1-\pi) \psi \Delta^{2}\right]}, 1\right] & \text { if } \quad(\widehat{\delta}=0, \widehat{\rho}=1)
\end{array}\right.
$$

The Selectorate After knowing their first-period utility at the end of the first period, the selectorate chooses between $\operatorname{coup}(\rho=0)$ or not $(\rho=1)$. This means that to derive the selectorate's sequential rational behavior, we should consider four possible information sets, where in each information set, there are two decision nodes depending on the type of leader. Let $V^{S}\left(\rho=1, \alpha^{B R} \mid \widehat{\delta}\right)$ be the expected continuation payoff for the selectorate in $\widehat{\delta}$ if he subverts the incumbent leader and the citizens will subsequently choose according to $\alpha^{B R}$. Note that in this 
case, the payoff does not depend on $\widehat{\delta}$ being either 0 or $\Delta$, since the leader has been changed. Therefore, for any $\widehat{\delta} \in\left\{0, \Delta^{1}, \Delta^{2}, \Delta^{1}+\Delta^{2}\right\}$

$$
\begin{gathered}
V^{S}\left(\rho=1, \alpha^{B R} \mid \widehat{\delta}\right)=\left(1-\alpha^{B R}\right)\left[\pi \times\left(\Delta^{1}+\Delta^{2}\right)+(1-\pi) \times \psi \Delta^{2}+\phi \times \frac{X}{\phi}+(1-\phi) \times 0\right]+\alpha^{B R} \times 0= \\
=\left(1-\alpha^{B R}\right)\left[\pi\left(\Delta^{1}+\Delta^{2}\right)+(1-\pi) \psi \Delta^{2}+X\right]:
\end{gathered}
$$

as the type of the newly picked up leader is unknown, she will produce $\Delta^{1}+\Delta^{2}$ with probability $\pi$ and $\psi \Delta^{2}$ (in expected terms) otherwise. Moreover, both types of leader will distribute the entire social revenue to the selectorate, but the members of the selectorate who ousted the leader, with probability $\phi$, will be included in the successor's selectorate getting the patronage $\frac{X}{\phi}$ in the second period. On the other hand

$$
\begin{gathered}
V^{S}\left(\rho=0, \alpha^{B R} \mid \widehat{\delta}\right)=\left(1-\alpha^{B R}\right)\left[\mu(C \mid \widehat{\delta}, \widehat{\rho}=0) \times\left(\Delta^{1}+\Delta^{2}\right)+[1-\mu(C \mid \widehat{\delta}, \widehat{\rho}=0)] \times \psi \Delta^{2}+\frac{X}{\phi}\right]+\alpha^{B R} \times 0= \\
=\left(1-\alpha^{B R}\right)\left[\mu(C \mid \widehat{\delta}, \widehat{\rho}=0)\left(\Delta^{1}+\Delta^{2}\right)+[1-\mu(C \mid \widehat{\delta}, \widehat{\rho}=0)] \psi \Delta^{2}+\frac{X}{\phi}\right] .
\end{gathered}
$$

Let consider the four regions in the space $\zeta \in[0,1]$ :

1. $\zeta \in\left[\frac{1}{X-\left(\Delta^{1}+\Delta^{2}\right)}, 1\right]:$ then, considering the four selectorate's information sets:

(a) in $\left(\widehat{\delta}=\Delta^{1}+\Delta^{2}\right)$ the selectorate's beliefs are

$$
\mu\left(C \mid \widehat{\delta}=\Delta^{1}+\Delta^{2}\right)=\frac{\pi\left[\bar{\lambda}^{C, 1} \bar{\lambda}^{C, 2}+\bar{\lambda}^{C, 1} \psi\left(1-\bar{\lambda}^{C, 2}\right)\right]}{\pi\left[\bar{\lambda}^{C, 1} \bar{\lambda}^{C, 2}+\lambda \psi\left(1-\bar{\lambda}^{C, 2}\right)\right]+(1-\pi)\left[\bar{\lambda}^{N, 1} \bar{\lambda}^{N, 2}+\bar{\lambda}^{N, 1} \psi\left(1-\bar{\lambda}^{N, 2}\right)\right]}=1
$$

and

$$
\begin{aligned}
& \rho\left(\alpha^{B R} \mid \widehat{\delta}=\Delta^{1}+\Delta^{2}\right)=0 \Leftrightarrow V^{S}\left(\rho=0, \alpha^{B R} \mid \widehat{\delta}=\Delta^{1}+\Delta^{2}\right) \geq V^{S}\left(\rho=1, \alpha^{B R} \mid \widehat{\delta}=\Delta^{1}+\Delta^{2}\right) \Leftrightarrow \\
& \Leftrightarrow\left(1-\alpha^{B R}\right)\left[\left(\Delta^{1}+\Delta^{2}\right)+\frac{X}{\phi}\right] \geq\left(1-\alpha^{B R}\right)\left[\pi\left(\Delta^{1}+\Delta^{2}\right)+(1-\pi) \psi \Delta^{2}+X\right] \Leftrightarrow 0 \geq 0
\end{aligned}
$$

which, in turn, implies the following sequential best reply correspondence when $\widehat{\delta}=$ $\Delta^{1}+\Delta^{2}$

$$
\rho\left(\widehat{\delta}=\Delta^{1}+\Delta^{2} \mid \alpha^{B R}\right)^{B R} \in[0,1]
$$

(b) in $\left(\widehat{\delta}=\Delta^{1}\right)$ the selectorate's beliefs are

$$
\mu\left(C \mid \widehat{\delta}=\Delta^{1}\right)=\frac{\pi\left[\bar{\lambda}^{C, 1}(1-\psi)\left(1-\bar{\lambda}^{C, 2}\right)\right]}{\pi\left[\bar{\lambda}^{C, 1}(1-\psi)\left(1-\bar{\lambda}^{C, 2}\right)\right]+(1-\pi)\left[\bar{\lambda}^{N, 1}(1-\psi)\left(1-\bar{\lambda}^{N, 2}\right)\right]} \in[0,1]=0
$$


and

$$
\begin{gathered}
\rho\left(\alpha^{B R} \mid \widehat{\delta}=\Delta^{1}\right)=0 \Leftrightarrow V^{S}\left(\rho=0, \alpha^{B R} \mid \widehat{\delta}=\Delta^{1}\right) \geq V^{S}\left(\rho=1, \alpha^{B R} \mid \widehat{\delta}=\Delta^{1}\right) \Leftrightarrow \\
\Leftrightarrow\left(1-\alpha^{B R}\right)\left[\psi \Delta^{2}+\frac{X}{\phi}\right] \geq\left(1-\alpha^{B R}\right)\left[\pi\left(\Delta^{1}+\Delta^{2}\right)+(1-\pi) \psi \Delta^{2}+X\right] \Leftrightarrow 0 \geq 0
\end{gathered}
$$

which, in turn, implies the following sequential best reply correspondence when $\widehat{\delta}=\Delta^{1}$

$$
\rho\left(\widehat{\delta}=\Delta^{1} \mid \alpha^{B R}\right)^{B R} \in[0,1]
$$

(c) in $\left(\widehat{\delta}=\Delta^{2}\right)$ the selectorate's beliefs are

$$
\begin{gathered}
\mu\left(C \mid \widehat{\delta}=\Delta^{2}\right)= \\
=\frac{\pi\left[\left(1-\bar{\lambda}^{C, 1}\right) \bar{\lambda}^{C, 2}+\left(1-\bar{\lambda}^{C, 1}\right) \psi\left(1-\bar{\lambda}^{C, 2}\right)\right]}{\pi\left[\left(1-\bar{\lambda}^{C, 1}\right) \bar{\lambda}^{C, 2}+\left(1-\bar{\lambda}^{C, 1}\right) \psi\left(1-\bar{\lambda}^{C, 2}\right)\right]+(1-\pi)\left[\left(1-\bar{\lambda}^{N, 1}\right) \bar{\lambda}^{N, 2}+\left(1-\bar{\lambda}^{N, 1}\right) \psi\left(1-\bar{\lambda}^{N, 2}\right)\right]}
\end{gathered}
$$

and

$$
\begin{gathered}
\rho\left(\alpha^{B R} \mid \widehat{\delta}=\Delta^{2}\right)=0 \Leftrightarrow V^{S}\left(\rho=0, \alpha^{B R} \mid \widehat{\delta}=\Delta^{2}\right) \geq V^{S}\left(\rho=1, \alpha^{B R} \mid \widehat{\delta}=\Delta^{2}\right) \Leftrightarrow \\
\Leftrightarrow\left(1-\alpha^{B R}\right)\left[\psi \Delta^{2}+\frac{X}{\phi}\right] \geq\left(1-\alpha^{B R}\right)\left[\pi\left(\Delta^{1}+\Delta^{2}\right)+(1-\pi) \psi \Delta^{2}+X\right] \Leftrightarrow 0 \geq 0
\end{gathered}
$$

which, in turn, implies the following sequential best reply correspondence when $\widehat{\delta}=\Delta^{1}$

$$
\rho\left(\widehat{\delta}=\Delta^{2} \mid \alpha^{B R}\right)^{B R} \in[0,1]
$$

(d) in $(\widehat{\delta}=0)$ the selectorate's beliefs are

$$
\mu(C \mid \widehat{\delta}=0)=\frac{\pi\left[\left(1-\bar{\lambda}^{C, 1}\right)(1-\psi)\left(1-\bar{\lambda}^{C, 2}\right)\right]}{\pi\left[\left(1-\bar{\lambda}^{C, 1}\right)(1-\psi)\left(1-\bar{\lambda}^{C, 2}\right)\right]+(1-\pi)\left[\left(1-\bar{\lambda}^{N, 1}\right)(1-\psi)\left(1-\bar{\lambda}^{N, 2}\right)\right]}=0
$$

and

$$
\begin{gathered}
\rho\left(\alpha^{B R} \mid \widehat{\delta}=0\right)=0 \Leftrightarrow V^{S}\left(\rho=0, \alpha^{B R} \mid \widehat{\delta}=0\right) \geq V^{S}\left(\rho=1, \alpha^{B R} \mid \widehat{\delta}=0\right) \Leftrightarrow \\
\Leftrightarrow\left(1-\alpha^{B R}\right)\left[\psi \Delta^{2}+\frac{X}{\phi}\right] \geq\left(1-\alpha^{B R}\right)\left[\pi\left(\Delta^{1}+\Delta^{2}\right)+(1-\pi) \psi \Delta^{2}+X\right] \Leftrightarrow 0 \geq 0
\end{gathered}
$$

which, in turn, implies the following sequential best reply correspondence when $\widehat{\delta}=\Delta^{1}$

$$
\rho\left(\widehat{\delta}=0 \mid \alpha^{B R}\right)^{B R} \in[0,1]
$$


2. $\zeta \in\left[\frac{1}{X-\left[\pi\left(\Delta^{1}+\Delta^{2}\right)+(1-\pi) \psi \Delta^{2}\right]}, \frac{1}{X-\left(\Delta^{1}+\Delta^{2}\right)}\right]:$ then, considering the four selectorate's information sets:

(a) in $\left(\widehat{\delta}=\Delta^{1}+\Delta^{2}\right)$ the selectorate's beliefs are

$$
\mu\left(C \mid \widehat{\delta}=\Delta^{1}+\Delta^{2}\right)=\frac{\pi\left[\bar{\lambda}^{C, 1} \bar{\lambda}^{C, 2}+\bar{\lambda}^{C, 1} \psi\left(1-\bar{\lambda}^{C, 2}\right)\right]}{\pi\left[\bar{\lambda}^{C, 1} \bar{\lambda}^{C, 2}+\lambda \psi\left(1-\bar{\lambda}^{C, 2}\right)\right]+(1-\pi)\left[\bar{\lambda}^{N, 1} \bar{\lambda}^{N, 2}+\bar{\lambda}^{N, 1} \psi\left(1-\bar{\lambda}^{N, 2}\right)\right]}=1
$$

and

$\rho\left(\alpha^{B R} \mid \widehat{\delta}=\Delta^{1}+\Delta^{2}\right)=0 \Leftrightarrow V^{S}\left(\rho=0, \alpha^{B R} \mid \widehat{\delta}=\Delta^{1}+\Delta^{2}\right) \geq V^{S}\left(\rho=1, \alpha^{B R} \mid \widehat{\delta}=\Delta^{1}+\Delta^{2}\right) \Leftrightarrow$

$\Leftrightarrow\left(1-\alpha^{B R}\right)\left[\left(\Delta^{1}+\Delta^{2}\right)+\frac{X}{\phi}\right] \geq\left(1-\alpha^{B R}\right)\left[\pi\left(\Delta^{1}+\Delta^{2}\right)+(1-\pi) \psi \Delta^{2}+X\right] \Leftrightarrow\left[\left(\Delta^{1}+\Delta^{2}\right)+\frac{X}{\phi}\right] \geq$

which, in turn, implies the following sequential best reply correspondence when $\widehat{\delta}=$

$\Delta^{1}+\Delta^{2}$

$$
\rho\left(\widehat{\delta}=\Delta^{1}+\Delta^{2} \mid \alpha^{B R}\right)^{B R}=0
$$

(b) in $\left(\widehat{\delta}=\Delta^{1}\right)$ the selectorate's beliefs are

$$
\mu\left(C \mid \widehat{\delta}=\Delta^{1}\right)=\frac{\pi\left[\bar{\lambda}^{C, 1}(1-\psi)\left(1-\bar{\lambda}^{C, 2}\right)\right]}{\pi\left[\bar{\lambda}^{C, 1}(1-\psi)\left(1-\bar{\lambda}^{C, 2}\right)\right]+(1-\pi)\left[\bar{\lambda}^{N, 1}(1-\psi)\left(1-\bar{\lambda}^{N, 2}\right)\right]} \in[0,1]=0
$$

and

$$
\begin{gathered}
\rho\left(\alpha^{B R} \mid \widehat{\delta}=\Delta^{1}\right)=0 \Leftrightarrow V^{S}\left(\rho=0, \alpha^{B R} \mid \widehat{\delta}=\Delta^{1}\right) \geq V^{S}\left(\rho=1, \alpha^{B R} \mid \widehat{\delta}=\Delta^{1}\right) \Leftrightarrow \\
\Leftrightarrow\left(1-\alpha^{B R}\right)\left[\psi \Delta^{2}+\frac{X}{\phi}\right] \geq\left(1-\alpha^{B R}\right)\left[\pi\left(\Delta^{1}+\Delta^{2}\right)+(1-\pi) \psi \Delta^{2}+X\right] \Leftrightarrow 0 \geq 0
\end{gathered}
$$

which, in turn, implies the following sequential best reply correspondence when $\widehat{\delta}=\Delta^{1}$

$$
\rho\left(\widehat{\delta}=\Delta^{1} \mid \alpha^{B R}\right)^{B R} \in[0,1]
$$

(c) in $\left(\widehat{\delta}=\Delta^{2}\right)$ the selectorate's beliefs are

$$
\begin{gathered}
\mu\left(C \mid \widehat{\delta}=\Delta^{2}\right)= \\
=\frac{\pi\left[\left(1-\bar{\lambda}^{C, 1}\right) \bar{\lambda}^{C, 2}+\left(1-\bar{\lambda}^{C, 1}\right) \psi\left(1-\bar{\lambda}^{C, 2}\right)\right]}{\pi\left[\left(1-\bar{\lambda}^{C, 1}\right) \bar{\lambda}^{C, 2}+\left(1-\bar{\lambda}^{C, 1}\right) \psi\left(1-\bar{\lambda}^{C, 2}\right)\right]+(1-\pi)\left[\left(1-\bar{\lambda}^{N, 1}\right) \bar{\lambda}^{N, 2}+\left(1-\bar{\lambda}^{N, 1}\right) \psi\left(1-\bar{\lambda}^{N, 2}\right)\right]}
\end{gathered}
$$

and

$$
\rho\left(\alpha^{B R} \mid \widehat{\delta}=\Delta^{2}\right)=0 \Leftrightarrow V^{S}\left(\rho=0, \alpha^{B R} \mid \widehat{\delta}=\Delta^{2}\right) \geq V^{S}\left(\rho=1, \alpha^{B R} \mid \widehat{\delta}=\Delta^{2}\right) \Leftrightarrow
$$


$\Leftrightarrow\left(1-\alpha^{B R}\right)\left[\psi \Delta^{2}+\frac{X}{\phi}\right] \geq\left(1-\alpha^{B R}\right)\left[\pi\left(\Delta^{1}+\Delta^{2}\right)+(1-\pi) \psi \Delta^{2}+X\right] \Leftrightarrow 0 \geq 0$

which, in turn, implies the following sequential best reply correspondence when $\widehat{\delta}=\Delta^{2}$

$$
\rho\left(\widehat{\delta}=\Delta^{2} \mid \alpha^{B R}\right)^{B R} \in[0,1]
$$

(d) in $(\widehat{\delta}=0)$ the selectorate's beliefs are

$$
\mu(C \mid \widehat{\delta}=0)=\frac{\pi\left[\left(1-\bar{\lambda}^{C, 1}\right)(1-\psi)\left(1-\bar{\lambda}^{C, 2}\right)\right]}{\pi\left[\left(1-\bar{\lambda}^{C, 1}\right)(1-\psi)\left(1-\bar{\lambda}^{C, 2}\right)\right]+(1-\pi)\left[\left(1-\bar{\lambda}^{N, 1}\right)(1-\psi)\left(1-\bar{\lambda}^{N, 2}\right)\right]}=0
$$

and

$$
\begin{gathered}
\rho\left(\alpha^{B R} \mid \widehat{\delta}=0\right)=0 \Leftrightarrow V^{S}\left(\rho=0, \alpha^{B R} \mid \widehat{\delta}=0\right) \geq V^{S}\left(\rho=1, \alpha^{B R} \mid \widehat{\delta}=0\right) \Leftrightarrow \\
\Leftrightarrow\left(1-\alpha^{B R}\right)\left[\psi \Delta^{2}+\frac{X}{\phi}\right] \geq\left(1-\alpha^{B R}\right)\left[\pi\left(\Delta^{1}+\Delta^{2}\right)+(1-\pi) \psi \Delta^{2}+X\right] \Leftrightarrow 0 \geq 0
\end{gathered}
$$

which, in turn, implies the following sequential best reply correspondence when $\widehat{\delta}=0$

$$
\rho\left(\widehat{\delta}=0 \mid \alpha^{B R}\right)^{B R} \in[0,1]
$$

3. $\zeta \in\left[\frac{1}{X-\psi \Delta^{2}}, \frac{1}{X-\left[\pi\left(\Delta^{1}+\Delta^{2}\right)+(1-\pi) \psi \Delta^{2}\right]}\right]$ : then, considering the four selectorate's information sets:

(a) in $\left(\widehat{\delta}=\Delta^{1}+\Delta^{2}\right)$ the selectorate's beliefs are

$$
\mu\left(C \mid \widehat{\delta}=\Delta^{1}+\Delta^{2}\right)=\frac{\pi\left[\bar{\lambda}^{C, 1} \bar{\lambda}^{C, 2}+\bar{\lambda}^{C, 1} \psi\left(1-\bar{\lambda}^{C, 2}\right)\right]}{\pi\left[\bar{\lambda}^{C, 1} \bar{\lambda}^{C, 2}+\lambda \psi\left(1-\bar{\lambda}^{C, 2}\right)\right]+(1-\pi)\left[\bar{\lambda}^{N, 1} \bar{\lambda}^{N, 2}+\bar{\lambda}^{N, 1} \psi\left(1-\bar{\lambda}^{N, 2}\right)\right]}=1
$$

and

$$
\begin{gathered}
\rho\left(\alpha^{B R} \mid \widehat{\delta}=\Delta^{1}+\Delta^{2}\right)=0 \Leftrightarrow V^{S}\left(\rho=0, \alpha^{B R} \mid \widehat{\delta}=\Delta^{1}+\Delta^{2}\right) \geq V^{S}\left(\rho=1, \alpha^{B R} \mid \widehat{\delta}=\Delta^{1}+\Delta^{2}\right) \Leftrightarrow \\
\Leftrightarrow\left(\Delta^{1}+\Delta^{2}\right)+\frac{X}{\phi} \geq \pi\left(\Delta^{1}+\Delta^{2}\right)+(1-\pi) \psi \Delta^{2}+X
\end{gathered}
$$

which is always satisfied, hence it implies the following sequential best reply correspondence when $\widehat{\delta}=\Delta^{1}+\Delta^{2}$

$$
\rho\left(\widehat{\delta}=\Delta^{1}+\Delta^{2} \mid \alpha^{B R}\right)^{B R}=0
$$


(b) in $\left(\widehat{\delta}=\Delta^{1}\right)$ the selectorate's beliefs are

$$
\mu\left(C \mid \widehat{\delta}=\Delta^{1}\right)=\frac{\pi\left[\bar{\lambda}^{C, 1}(1-\psi)\left(1-\bar{\lambda}^{C, 2}\right)\right]}{\pi\left[\bar{\lambda}^{C, 1}(1-\psi)\left(1-\bar{\lambda}^{C, 2}\right)\right]+(1-\pi)\left[\bar{\lambda}^{N, 1}(1-\psi)\left(1-\bar{\lambda}^{N, 2}\right)\right]} \in[0,1]=0
$$

and

$$
\begin{aligned}
\rho\left(\alpha^{B R} \mid \widehat{\delta}=\Delta^{1}\right)= & 0 \Leftrightarrow V^{S}\left(\rho=0, \alpha^{B R} \mid \widehat{\delta}=\Delta^{1}\right) \geq V^{S}\left(\rho=1, \alpha^{B R} \mid \widehat{\delta}=\Delta^{1}\right) \Leftrightarrow \\
& \Leftrightarrow 0 \geq \pi\left(\Delta^{1}+\Delta^{2}\right)+(1-\pi) \psi \Delta^{2}+X
\end{aligned}
$$

which, in turn, implies the following sequential best reply correspondence when $\widehat{\delta}=\Delta^{1}$

$$
\rho\left(\widehat{\delta}=\Delta^{1} \mid \alpha^{B R}\right)^{B R}=1
$$

(c) in $\left(\widehat{\delta}=\Delta^{2}\right)$ the selectorate's beliefs are

$$
\begin{gathered}
\mu\left(C \mid \widehat{\delta}=\Delta^{2}\right)= \\
=\frac{\pi\left[\left(1-\bar{\lambda}^{C, 1}\right) \bar{\lambda}^{C, 2}+\left(1-\bar{\lambda}^{C, 1}\right) \psi\left(1-\bar{\lambda}^{C, 2}\right)\right]}{\pi\left[\left(1-\bar{\lambda}^{C, 1}\right) \bar{\lambda}^{C, 2}+\left(1-\bar{\lambda}^{C, 1}\right) \psi\left(1-\bar{\lambda}^{C, 2}\right)\right]+(1-\pi)\left[\left(1-\bar{\lambda}^{N, 1}\right) \bar{\lambda}^{N, 2}+\left(1-\bar{\lambda}^{N, 1}\right) \psi\left(1-\bar{\lambda}^{N, 2}\right)\right]}
\end{gathered}
$$

and

$$
\begin{aligned}
\rho\left(\alpha^{B R} \mid \widehat{\delta}=\Delta^{2}\right)= & 0 \Leftrightarrow V^{S}\left(\rho=0, \alpha^{B R} \mid \widehat{\delta}=\Delta^{2}\right) \geq V^{S}\left(\rho=1, \alpha^{B R} \mid \widehat{\delta}=\Delta^{2}\right) \Leftrightarrow \\
& \Leftrightarrow 0 \geq\left[\pi\left(\Delta^{1}+\Delta^{2}\right)+(1-\pi) \psi \Delta^{2}+X\right]
\end{aligned}
$$

which, in turn, implies the following sequential best reply correspondence when $\widehat{\delta}=\Delta^{2}$

$$
\rho\left(\widehat{\delta}=\Delta^{2} \mid \alpha^{B R}\right)^{B R}=1
$$

(d) in $(\widehat{\delta}=0)$ the selectorate's beliefs are

$$
\mu(C \mid \widehat{\delta}=0)=\frac{\pi\left[\left(1-\bar{\lambda}^{C, 1}\right)(1-\psi)\left(1-\bar{\lambda}^{C, 2}\right)\right]}{\pi\left[\left(1-\bar{\lambda}^{C, 1}\right)(1-\psi)\left(1-\bar{\lambda}^{C, 2}\right)\right]+(1-\pi)\left[\left(1-\bar{\lambda}^{N, 1}\right)(1-\psi)\left(1-\bar{\lambda}^{N, 2}\right)\right]}=0
$$

and

$$
\begin{gathered}
\rho\left(\alpha^{B R} \mid \widehat{\delta}=0\right)=0 \Leftrightarrow V^{S}\left(\rho=0, \alpha^{B R} \mid \widehat{\delta}=0\right) \geq V^{S}\left(\rho=1, \alpha^{B R} \mid \widehat{\delta}=0\right) \Leftrightarrow \\
\Leftrightarrow 0 \geq\left(1-\alpha^{B R}\right)\left[\pi\left(\Delta^{1}+\Delta^{2}\right)+(1-\pi) \psi \Delta^{2}+X\right]
\end{gathered}
$$

which, in turn, implies the following sequential best reply correspondence when $\widehat{\delta}=0$

$$
\rho\left(\widehat{\delta}=0 \mid \alpha^{B R}\right)^{B R}=1
$$


4. $\zeta \in\left[0, \frac{1}{X-\psi \Delta^{2}}\right]$ : then, considering the four selectorate's information sets:

(a) in $\left(\widehat{\delta}=\Delta^{1}+\Delta^{2}\right)$ the selectorate's beliefs are

$$
\mu\left(C \mid \widehat{\delta}=\Delta^{1}+\Delta^{2}\right)=\frac{\pi\left[\bar{\lambda}^{C, 1} \bar{\lambda}^{C, 2}+\bar{\lambda}^{C, 1} \psi\left(1-\bar{\lambda}^{C, 2}\right)\right]}{\pi\left[\bar{\lambda}^{C, 1} \bar{\lambda}^{C, 2}+\lambda \psi\left(1-\bar{\lambda}^{C, 2}\right)\right]+(1-\pi)\left[\bar{\lambda}^{N, 1} \bar{\lambda}^{N, 2}+\bar{\lambda}^{N, 1} \psi\left(1-\bar{\lambda}^{N, 2}\right)\right]}=1
$$

and

$$
\begin{gathered}
\rho\left(\alpha^{B R} \mid \widehat{\delta}=\Delta^{1}+\Delta^{2}\right)=0 \Leftrightarrow V^{S}\left(\rho=0, \alpha^{B R} \mid \widehat{\delta}=\Delta^{1}+\Delta^{2}\right) \geq V^{S}\left(\rho=1, \alpha^{B R} \mid \widehat{\delta}=\Delta^{1}+\Delta^{2}\right) \Leftrightarrow \\
\Leftrightarrow\left(\Delta^{1}+\Delta^{2}\right)+\frac{X}{\phi} \geq \pi\left(\Delta^{1}+\Delta^{2}\right)+(1-\pi) \psi \Delta^{2}+X
\end{gathered}
$$

which, in turn, implies the following sequential best reply correspondence when $\widehat{\delta}=$

$\Delta^{1}+\Delta^{2}$

$$
\rho\left(\widehat{\delta}=\Delta^{1}+\Delta^{2} \mid \alpha^{B R}\right)^{B R}=0
$$

(b) in $\left(\widehat{\delta}=\Delta^{1}\right)$ the selectorate's beliefs are

$$
\mu\left(C \mid \widehat{\delta}=\Delta^{1}\right)=\frac{\pi\left[\bar{\lambda}^{C, 1}(1-\psi)\left(1-\bar{\lambda}^{C, 2}\right)\right]}{\pi\left[\bar{\lambda}^{C, 1}(1-\psi)\left(1-\bar{\lambda}^{C, 2}\right)\right]+(1-\pi)\left[\bar{\lambda}^{N, 1}(1-\psi)\left(1-\bar{\lambda}^{N, 2}\right)\right]} \in[0,1]=0
$$

and

$$
\begin{gathered}
\rho\left(\alpha^{B R} \mid \widehat{\delta}=\Delta^{1}\right)=0 \Leftrightarrow V^{S}\left(\rho=0, \alpha^{B R} \mid \widehat{\delta}=\Delta^{1}\right) \geq V^{S}\left(\rho=1, \alpha^{B R} \mid \widehat{\delta}=\Delta^{1}\right) \Leftrightarrow \\
\Leftrightarrow \frac{X}{\phi} \geq \pi\left(\Delta^{1}+\Delta^{2}\right)-\pi \psi \Delta^{2}+X \Leftrightarrow \phi \leq \frac{X}{\pi\left(\Delta^{1}+\Delta^{2}-\psi \Delta^{2}\right)+X}
\end{gathered}
$$

which, in turn, implies the following sequential best reply correspondence when $\widehat{\delta}=\Delta^{1}$

$$
\rho\left(\widehat{\delta}=\Delta^{1} \mid \alpha^{B R}\right)^{B R}=\left\{\begin{array}{lll}
0 & \text { if } \phi \in\left[0, \frac{X}{\pi\left(\Delta^{1}+\Delta^{2}-\psi \Delta^{2}\right)+X}\right] \\
1 & \text { if } \phi \in\left[\frac{X}{\pi\left(\Delta^{1}+\Delta^{2}-\psi \Delta^{2}\right)+X}, 1\right]
\end{array}\right.
$$

(c) in $\left(\widehat{\delta}=\Delta^{2}\right)$ the selectorate's beliefs are

$$
\begin{gathered}
\mu\left(C \mid \widehat{\delta}=\Delta^{2}\right)= \\
=\frac{\pi\left[\left(1-\bar{\lambda}^{C, 1}\right) \bar{\lambda}^{C, 2}+\left(1-\bar{\lambda}^{C, 1}\right) \psi\left(1-\bar{\lambda}^{C, 2}\right)\right]}{\pi\left[\left(1-\bar{\lambda}^{C, 1}\right) \bar{\lambda}^{C, 2}+\left(1-\bar{\lambda}^{C, 1}\right) \psi\left(1-\bar{\lambda}^{C, 2}\right)\right]+(1-\pi)\left[\left(1-\bar{\lambda}^{N, 1}\right) \bar{\lambda}^{N, 2}+\left(1-\bar{\lambda}^{N, 1}\right) \psi\left(1-\bar{\lambda}^{N, 2}\right)\right]}
\end{gathered}
$$

and

$$
\rho\left(\alpha^{B R} \mid \widehat{\delta}=\Delta^{2}\right)=0 \Leftrightarrow V^{S}\left(\rho=0, \alpha^{B R} \mid \widehat{\delta}=\Delta^{2}\right) \geq V^{S}\left(\rho=1, \alpha^{B R} \mid \widehat{\delta}=\Delta^{2}\right) \Leftrightarrow
$$




$$
\Leftrightarrow \psi \Delta^{2}+\frac{X}{\phi} \geq \pi\left(\Delta^{1}+\Delta^{2}\right)+(1-\pi) \psi \Delta^{2}+X \Leftrightarrow \phi \leq \frac{X}{\pi\left(\Delta^{1}+\Delta^{2}-\psi \Delta^{2}\right)+X}
$$

which, in turn, implies the following sequential best reply correspondence when $\widehat{\delta}=\Delta^{2}$

$$
\rho\left(\widehat{\delta}=\Delta^{2} \mid \alpha^{B R}\right)^{B R}=\left\{\begin{array}{lll}
0 & \text { if } & \phi \in\left[0, \frac{X}{\pi\left(\Delta^{1}+\Delta^{2}-\psi \Delta^{2}\right)+X}\right] \\
1 & \text { if } & \phi \in\left[\frac{X}{\pi\left(\Delta^{1}+\Delta^{2}-\psi \Delta^{2}\right)+X}, 1\right]
\end{array}\right.
$$

(d) in $(\widehat{\delta}=0)$ the selectorate's beliefs are

$$
\mu(C \mid \widehat{\delta}=0)=\frac{\pi\left[\left(1-\bar{\lambda}^{C, 1}\right)(1-\psi)\left(1-\bar{\lambda}^{C, 2}\right)\right]}{\pi\left[\left(1-\bar{\lambda}^{C, 1}\right)(1-\psi)\left(1-\bar{\lambda}^{C, 2}\right)\right]+(1-\pi)\left[\left(1-\bar{\lambda}^{N, 1}\right)(1-\psi)\left(1-\bar{\lambda}^{N, 2}\right)\right]}=0
$$

and

$$
\begin{gathered}
\rho\left(\alpha^{B R} \mid \widehat{\delta}=0\right)=0 \Leftrightarrow V^{S}\left(\rho=0, \alpha^{B R} \mid \widehat{\delta}=0\right) \geq V^{S}\left(\rho=1, \alpha^{B R} \mid \widehat{\delta}=0\right) \Leftrightarrow \\
\Leftrightarrow \psi \Delta^{2}+\frac{X}{\phi} \geq \pi\left(\Delta^{1}+\Delta^{2}\right)+(1-\pi) \psi \Delta^{2}+X \Leftrightarrow \phi \leq \frac{X}{\pi\left(\Delta^{1}+\Delta^{2}-\psi \Delta^{2}\right)+X}
\end{gathered}
$$

which, in turn, implies the following sequential best reply correspondence when $\widehat{\delta}=0$

$$
\rho\left(\widehat{\delta}=0 \mid \alpha^{B R}\right)^{B R}=\left\{\begin{array}{lll}
0 & \text { if } & \phi \in\left[0, \frac{X}{\pi\left(\Delta^{1}+\Delta^{2}-\psi \Delta^{2}\right)+X}\right] \\
1 & \text { if } & \phi \in\left[\frac{X}{\pi\left(\Delta^{1}+\Delta^{2}-\psi \Delta^{2}\right)+X}, 1\right] .
\end{array}\right.
$$

The Non Congruent Leader Now, we have to check whether the strategy profile $\left(\bar{\lambda}^{C, 1}, \bar{\lambda}^{C, 2}, \bar{\lambda}^{N, 1}, \bar{\lambda}^{N, 2}\right)=$ $(1,1,0,0)$ is part of of a SE. Her payoff will depend on $\rho^{B R}$ and $\alpha^{B R}$, hence on the basis of the previous $\alpha(\widehat{\delta}, \widehat{\rho})^{B R}$ and $\rho\left(\widehat{\delta} \mid \alpha^{B R}\right)^{B R}$ we need to distinguish five regions in the space $(\zeta, \phi) \in[0,1] \times[0,1]:$

1. if $(\zeta, \phi) \in\left[\frac{1}{X-\left(\Delta^{1}+\Delta^{2}\right)}, 1\right] \times[0,1]$ then

$$
V^{N}\left(1,1,0,0 ; \rho^{B R}, \alpha^{B R}\right)=\psi \Delta^{2}+r_{1}^{1}+r_{1}^{2}+\frac{X}{\phi}+0
$$

while deviating she get

$$
V^{N}\left(\bar{\lambda}^{C, 1}, \bar{\lambda}^{C, 2}, \bar{\lambda}^{N, 1}, \bar{\lambda}^{N, 2} ; \rho^{B R}, \alpha^{B R}\right)= \begin{cases}\psi \Delta^{2}+\Delta^{1}+r_{1}^{2}+\frac{X}{\phi}+0 & \text { if } \bar{\lambda}^{C, 1}, \bar{\lambda}^{C, 2}, \bar{\lambda}^{N, 1}, \bar{\lambda}^{N, 2}=1,1,1,0 \\ \Delta^{2}+r_{1}^{1}+\frac{X}{\phi}+0 & \text { if } \bar{\lambda}^{C, 1}, \bar{\lambda}^{C, 2}, \bar{\lambda}^{N, 1}, \bar{\lambda}^{N, 2}=1,1,0,1 \\ \Delta^{2}+\Delta^{1}+\frac{X}{\phi}+0 & \text { if } \bar{\lambda}^{C, 1}, \bar{\lambda}^{C, 2}, \bar{\lambda}^{N, 1}, \bar{\lambda}^{N, 2}=1,1,1,1\end{cases}
$$

which is always less, hence in this region we get an equilibrium 
2. $(\zeta, \phi) \in\left[\frac{1}{X-\left[\pi\left(\Delta^{1}+\Delta^{2}\right)+(1-\pi) \psi \Delta^{2}\right]}, \frac{1}{X-\left(\Delta^{1}+\Delta^{2}\right)}\right] \times[0,1]$ then

$$
V^{N}\left(1,1,0,0 ; \rho^{B R}, \alpha^{B R}\right)=\psi \Delta^{2}+r_{1}^{1}+r_{1}^{2}+\frac{X}{\phi}+0
$$

while deviating she get

$$
\begin{gathered}
V^{N}\left(\bar{\lambda}^{C, 1}, \bar{\lambda}^{C, 2}, \bar{\lambda}^{N, 1}, \bar{\lambda}^{N, 2} ; \rho^{B R}, \alpha^{B R}\right)= \\
= \begin{cases}\psi \Delta^{2}+\Delta^{1}+r_{1}^{2}+\frac{X}{\phi}+\psi\left[\psi \Delta^{2}+E\left(r_{2}^{1}\right)+E\left(r_{2}^{2}\right)+\frac{X}{\phi}\right] & \text { if } \bar{\lambda}^{C, 1}, \bar{\lambda}^{C, 2}, \bar{\lambda}^{N, 1}, \bar{\lambda}^{N, 2}=1,1,1,0 \\
\Delta^{2}+r_{1}^{1}+\frac{X}{\phi}+0 & \text { if } \bar{\lambda}^{C, 1}, \bar{\lambda}^{C, 2}, \bar{\lambda}^{N, 1}, \bar{\lambda}^{N, 2}=1,1,0,1 \\
\Delta^{2}+\Delta^{1}+\frac{X}{\phi}+\psi \Delta^{2}+E\left(r_{2}^{1}\right)+E\left(r_{2}^{2}\right)+\frac{X}{\phi} & \text { if } \bar{\lambda}^{C, 1}, \bar{\lambda}^{C, 2}, \bar{\lambda}^{N, 1}, \bar{\lambda}^{N, 2}=1,1,1,1 .\end{cases}
\end{gathered}
$$

Thus, the non congruent leader has no incentives to deviate if and only if

$$
\left\{\begin{array}{l}
r_{1}^{1} \geq \Delta^{1}+\psi\left[\psi \Delta^{2}+E\left(r_{2}^{1}\right)+E\left(r_{2}^{2}\right)+\frac{X}{\phi}\right] \\
r_{1}^{1}+r_{1}^{2} \geq \Delta^{2}+\Delta^{1}+E\left(r_{2}^{1}\right)+E\left(r_{2}^{2}\right)+\frac{X}{\phi}
\end{array}\right.
$$

hence in this region the leader might choose

$$
\left(\bar{\lambda}^{C, 1}, \bar{\lambda}^{C, 2}, \bar{\lambda}^{N, 1}, \bar{\lambda}^{N, 2}\right)=(1,1,0,0)
$$

with probability

$\mathbf{P}\left\{r_{1}^{1} \geq \Delta^{1}+\psi\left[\psi \Delta^{2}+E\left(r_{2}^{1}\right)+E\left(r_{2}^{2}\right)+\frac{X}{\phi}\right] \& r_{1}^{1}+r_{1}^{2} \geq \Delta^{2}+\Delta^{1}+E\left(r_{2}^{1}\right)+E\left(r_{2}^{2}\right)+\frac{X}{\phi}\right\} \equiv F I(\psi)$ however before to conclude we need to consider players' incentives with the other leader's strategies.

3. $(\zeta, \phi) \in\left[\frac{1}{X-\psi \Delta^{2}}, \frac{1}{X-\left[\pi\left(\Delta^{1}+\Delta^{2}\right)+(1-\pi) \psi \Delta^{2}\right]}\right] \times[0,1]$ then

$$
V^{N}\left(1,1,0,0 ; \rho^{B R}, \alpha^{B R}\right)=\psi \Delta^{2}+r_{1}^{1}+r_{1}^{2}+\frac{X}{\phi}+0
$$

while deviating she get

$$
\begin{gathered}
V^{N}\left(\bar{\lambda}^{C, 1}, \bar{\lambda}^{C, 2}, \bar{\lambda}^{N, 1}, \bar{\lambda}^{N, 2} ; \rho^{B R}, \alpha^{B R}\right)= \\
= \begin{cases}\psi \Delta^{2}+\Delta^{1}+r_{1}^{2}+\frac{X}{\phi}+\psi\left[\psi \Delta^{2}+E\left(r_{2}^{1}\right)+E\left(r_{2}^{2}\right)+\frac{X}{\phi}\right] & \text { if } \bar{\lambda}^{C, 1}, \bar{\lambda}^{C, 2}, \bar{\lambda}^{N, 1}, \bar{\lambda}^{N, 2}=1,1,1,0 \\
\Delta^{2}+r_{1}^{1}+\frac{X}{\phi}+0 & \text { if } \bar{\lambda}^{C, 1}, \bar{\lambda}^{C, 2}, \bar{\lambda}^{N, 1}, \bar{\lambda}^{N, 2}=1,1,0,1 \\
\Delta^{2}+\Delta^{1}+\frac{X}{\phi}+\psi \Delta^{2}+E\left(r_{2}^{1}\right)+E\left(r_{2}^{2}\right)+\frac{X}{\phi} & \text { if } \bar{\lambda}^{C, 1}, \bar{\lambda}^{C, 2}, \bar{\lambda}^{N, 1}, \bar{\lambda}^{N, 2}=1,1,1,1 .\end{cases}
\end{gathered}
$$

Thus, the non congruent leader has no incentives to deviate if and only if

$$
\left\{\begin{array}{l}
r_{1}^{1} \geq \Delta^{1}+\psi\left[\psi \Delta^{2}+E\left(r_{2}^{1}\right)+E\left(r_{2}^{2}\right)+\frac{X}{\phi}\right] \\
r_{1}^{1}+r_{1}^{2} \geq \Delta^{2}+\Delta^{1}+E\left(r_{2}^{1}\right)+E\left(r_{2}^{2}\right)+\frac{X}{\phi}
\end{array}\right.
$$


hence in this region the leader might choose:

$$
\left(\bar{\lambda}^{C, 1}, \bar{\lambda}^{C, 2}, \bar{\lambda}^{N, 1}, \bar{\lambda}^{N, 2}\right)=(1,1,0,0)
$$

with probability

$$
\mathbf{P}\left\{r_{1}^{1} \geq \Delta^{1}+\psi\left[\psi \Delta^{2}+E\left(r_{2}^{1}\right)+E\left(r_{2}^{2}\right)+\frac{X}{\phi}\right] \& r_{1}^{1}+r_{1}^{2} \geq \Delta^{2}+\Delta^{1}+E\left(r_{2}^{1}\right)+E\left(r_{2}^{2}\right)+\frac{X}{\phi}\right\} \equiv F I(\psi)
$$

however before to conclude we need to consider players' incentives with the other leader's strategies.

4. $(\zeta, \phi) \in\left[0, \frac{1}{X-\psi \Delta^{2}}\right] \times\left[0, \frac{X}{\pi\left(\Delta^{1}+\Delta^{2}-\psi \Delta^{2}\right)+X}\right]$ thus

$$
V^{N}\left(1,1,0,0 ; \rho^{B R}, \alpha^{B R}\right)=\psi \Delta^{2}+r_{1}^{1}+r_{1}^{2}+\frac{X}{\phi}+\psi \Delta^{2}+E\left(r_{2}^{1}\right)+E\left(r_{2}^{2}\right)+\frac{X}{\phi}
$$

while deviating she get

$$
\begin{gathered}
V^{N}\left(\bar{\lambda}^{C, 1}, \bar{\lambda}^{C, 2}, \bar{\lambda}^{N, 1}, \bar{\lambda}^{N, 2} ; \rho^{B R}, \alpha^{B R}\right)= \\
= \begin{cases}\psi \Delta^{2}+\Delta^{1}+r_{1}^{2}+\frac{X}{\phi}+\psi \Delta^{2}+E\left(r_{2}^{1}\right)+E\left(r_{2}^{2}\right)+\frac{X}{\phi} & \text { if } \bar{\lambda}^{C, 1}, \bar{\lambda}^{C, 2}, \bar{\lambda}^{N, 1}, \bar{\lambda}^{N, 2}=1,1,1,0 \\
\Delta^{2}+r_{1}^{1}+\frac{X}{\phi}+\psi \Delta^{2}+E\left(r_{2}^{1}\right)+E\left(r_{2}^{2}\right)+\frac{X}{\phi} & \text { if } \bar{\lambda}^{C, 1}, \bar{\lambda}^{C, 2}, \bar{\lambda}^{N, 1}, \bar{\lambda}^{N, 2}=1,1,0,1 \\
\Delta^{2}+\Delta^{1}+\frac{X}{\phi}+\psi \Delta^{2}+E\left(r_{2}^{1}\right)+E\left(r_{2}^{2}\right)+\frac{X}{\phi} & \text { if } \bar{\lambda}^{C, 1}, \bar{\lambda}^{C, 2}, \bar{\lambda}^{N, 1}, \bar{\lambda}^{N, 2}=1,1,1,1 .\end{cases}
\end{gathered}
$$

Then, the non congruent leader has no incentives to deviate, hence in this region we have a unique equilibrium

5. $(\zeta, \phi) \in\left[0, \frac{1}{X-\psi \Delta^{2}}\right] \times\left[\frac{X}{\pi\left(\Delta^{1}+\Delta^{2}\right)+X}, 1\right]$ thus

$$
V^{N}\left(1,1,0,0 ; \rho^{B R}, \alpha^{B R}\right)=\psi \Delta^{2}+r_{1}^{1}+r_{1}^{2}+\frac{X}{\phi}+0
$$

while deviating she get

$$
\begin{gathered}
V^{N}\left(\bar{\lambda}^{C, 1}, \bar{\lambda}^{C, 2}, \bar{\lambda}^{N, 1}, \bar{\lambda}^{N, 2} ; \rho^{B R}, \alpha^{B R}\right)= \\
= \begin{cases}\psi \Delta^{2}+\Delta^{1}+r_{1}^{2}+\frac{X}{\phi}+\psi\left[\psi \Delta^{2}+E\left(r_{2}^{1}\right)+E\left(r_{2}^{2}\right)+\frac{X}{\phi}\right] & \text { if } \bar{\lambda}^{C, 1}, \bar{\lambda}^{C, 2}, \bar{\lambda}^{N, 1}, \bar{\lambda}^{N, 2}=1,1,1,0 \\
\Delta^{2}+r_{1}^{1}+\frac{X}{\phi}+0 & \text { if } \bar{\lambda}^{C, 1}, \bar{\lambda}^{C, 2}, \bar{\lambda}^{N, 1}, \bar{\lambda}^{N, 2}=1,1,0,1 \\
\Delta^{2}+\Delta^{1}+\frac{X}{\phi}+\psi \Delta^{2}+E\left(r_{2}^{1}\right)+E\left(r_{2}^{2}\right)+\frac{X}{\phi} & \text { if } \bar{\lambda}^{C, 1}, \bar{\lambda}^{C, 2}, \bar{\lambda}^{N, 1}, \bar{\lambda}^{N, 2}=1,1,1,1 .\end{cases}
\end{gathered}
$$

Then, the non congruent leader has no incentives to deviate if and only if

$$
\left\{\begin{array}{l}
r_{1}^{1} \geq \Delta^{1}+\psi\left[\psi \Delta^{2}+E\left(r_{2}^{1}\right)+E\left(r_{2}^{2}\right)+\frac{X}{\phi}\right] \\
r_{1}^{1}+r_{1}^{2} \geq \Delta^{2}+\Delta^{1}+E\left(r_{2}^{1}\right)+E\left(r_{2}^{2}\right)+\frac{X}{\phi}
\end{array}\right.
$$


Note that

$$
\Delta^{2}+\Delta^{1}+E\left(r_{2}^{1}\right)+E\left(r_{2}^{2}\right)+\frac{X}{\phi}>\Delta^{1}+\psi\left[\psi \Delta^{2}+E\left(r_{2}^{1}\right)+E\left(r_{2}^{2}\right)+\frac{X}{\phi}\right]
$$

hence in this region we may get an equilibrium:

$$
\left(\bar{\lambda}^{C, 1}, \bar{\lambda}^{C, 2}, \bar{\lambda}^{N, 1}, \bar{\lambda}^{N, 2}\right)=(1,1,0,0)
$$

with probability

$\mathbf{P}\left\{r_{1}^{1} \geq \Delta^{1}+\psi\left[\psi \Delta^{2}+E\left(r_{2}^{1}\right)+E\left(r_{2}^{2}\right)+\frac{X}{\phi}\right] \& r_{1}^{1}+r_{1}^{2} \geq \Delta^{2}+\Delta^{1}+E\left(r_{2}^{1}\right)+E\left(r_{2}^{2}\right)+\frac{X}{\phi}\right\} \equiv F I(\psi)$

however before to conclude we need to consider players' incentives with the other leader's strategies.

2. Strategy profile $\left(\bar{\lambda}^{C, 1}, \bar{\lambda}^{C, 2}, \bar{\lambda}^{N, 1}, \bar{\lambda}^{N, 2}\right)=(1,1,1,0)$

The Citizens After knowing their first-period utility and the selectorate's choice at the end of the first period, the citizens choose between revolt $(\alpha=1)$ or not $(\alpha=0)$. This means that to derive the citizens' sequential rational behavior, we should consider eight possible information sets, where in each information set, there are two decision nodes depending on the type of leader. Let $V^{Z}(\alpha \mid \delta, \rho)$ be the expected continuation payoff for the citizens when they choose $\alpha$ if $(\delta, \rho)$ has been observed. The expected continuation utility that the citizens will get after they choose to initiate a revolution in $(\delta, \rho)$ is:

$$
V^{Z}(\alpha=1 \mid \delta, \rho)=(1-\phi) \times \frac{X-(\zeta)^{-1}}{1-\phi}+\phi \times 0=X-(\zeta)^{-1} .
$$

Clearly, this payoff does not depend on their beliefs about the leader's type and thus on $(\widehat{\delta}, \rho)$. On the other hand, if the citizens decide to accommodate, the continuation payoff will depend on their beliefs about the type of leader which, in turn, will depend on their information at the time of deciding. Therefore, to find the citizens' rational behavior, we need to consider eight possible information sets and the citizens' beliefs in these information sets.

1. Information set $\left(\widehat{\delta}=\Delta^{1}+\Delta^{2}, \widehat{\rho}=0\right)$ In this information set the players' beliefs are $\mu\left(C \mid \widehat{\delta}=\Delta^{1}+\Delta^{2}\right)=\frac{\pi\left[\bar{\lambda}^{C, 1} \bar{\lambda}^{C, 2}+\bar{\lambda}^{C, 1} \psi\left(1-\bar{\lambda}^{C, 2}\right)\right]}{\pi\left[\bar{\lambda}^{C, 1} \bar{\lambda}^{C, 2}+\lambda \psi\left(1-\bar{\lambda}^{C, 2}\right)\right]+(1-\pi)\left[\bar{\lambda}^{N, 1} \bar{\lambda}^{N, 2}+\bar{\lambda}^{N, 1} \psi\left(1-\bar{\lambda}^{N, 2}\right)\right]}=\frac{\pi}{\pi+(1-\pi) \psi} \equiv \pi^{+}$ 
The expected continuation payoff the citizens will get after they choose not to revolt is

$$
\begin{aligned}
V^{Z}(\alpha=0 \mid \widehat{\delta} & \left.=\Delta^{1}+\Delta^{2}, \widehat{\rho}=0\right)=\mu\left(C \mid \widehat{\delta}=\Delta^{1}+\Delta^{2}\right)\left(\Delta^{1}+\Delta^{2}\right)+\left[1-\mu\left(C \mid \widehat{\delta}=\Delta^{1}+\Delta^{2}\right)\right] \psi \Delta^{2}= \\
& =\frac{\pi\left(\Delta^{1}+\Delta^{2}\right)+[(1-\pi) \psi] \psi \Delta^{2}}{\pi+(1-\pi) \psi}=\pi^{+}\left(\Delta^{1}+\Delta^{2}\right)+\left(1-\pi^{+}\right) \psi \Delta^{2}
\end{aligned}
$$

then sequential rationality implies that the citizens will choose to revolt in $\left(\widehat{\delta}=\Delta^{1}+\Delta^{2}, \widehat{\rho}=0\right)$ if and only if

$$
\begin{gathered}
V^{Z}\left(\alpha=1 \mid \widehat{\delta}=\Delta^{1}+\Delta^{2}, \widehat{\rho}=0\right) \geq V^{Z}\left(\alpha=0 \mid \widehat{\delta}=\Delta^{1}+\Delta^{2}, \widehat{\rho}=0\right) \Leftrightarrow \\
\Leftrightarrow \alpha\left(\widehat{\delta}=\Delta^{1}+\Delta^{2}, \widehat{\rho}=0\right)=1 \Leftrightarrow(\zeta)^{-1} \leq X-\left[\pi^{+}\left(\Delta^{1}+\Delta^{2}\right)+\left(1-\pi^{+}\right) \psi \Delta^{2}\right] .
\end{gathered}
$$

2. Information set $\left(\widehat{\delta}=\Delta^{1}+\Delta^{2}, \widehat{\rho}=1\right)$ In this information set, the incumbent leader is removed from office by the selectorate; therefore, there is a new leader and thus the expected utility the citizens will get after they choose not to revolt does not depend on the previous observation on $\widehat{\delta}$. Then, the expected utility the citizens will get after they choose not to revolt is:

$$
V^{Z}\left(\alpha=0 \mid \widehat{\delta}=\Delta^{1}+\Delta^{2}, \widehat{\rho}=1\right)=\pi\left(\Delta^{1}+\Delta^{2}\right)+(1-\pi) \psi \Delta^{2} .
$$

Sequential rationality implies that the citizens will choose to revolt in $\left(\widehat{\delta}=\Delta^{1}+\Delta^{2}, \widehat{\rho}=1\right)$ if and only if

$$
\begin{gathered}
V^{Z}\left(\alpha=1 \mid \widehat{\delta}=\Delta^{1}+\Delta^{2}, \widehat{\rho}=1\right) \geq V^{Z}\left(\alpha=0 \mid \widehat{\delta}=\Delta^{1}+\Delta^{2}, \widehat{\rho}=1\right) \\
\Leftrightarrow \alpha\left(\widehat{\delta}=\Delta^{1}+\Delta^{2}, \widehat{\rho}=1\right)=1 \Leftrightarrow(\zeta)^{-1} \leq X-\left[\pi\left(\Delta^{1}+\Delta^{2}\right)+(1-\pi) \psi \Delta^{2}\right] .
\end{gathered}
$$

3. Information set $\left(\widehat{\delta}=\Delta^{1}, \widehat{\rho}=0\right)$ In this information set the players' beliefs are

$$
\mu\left(C \mid \widehat{\delta}=\Delta^{1}\right)=\frac{\pi\left[\bar{\lambda}^{C, 1}(1-\psi)\left(1-\bar{\lambda}^{C, 2}\right)\right]}{\pi\left[\bar{\lambda}^{C, 1}(1-\psi)\left(1-\bar{\lambda}^{C, 2}\right)\right]+(1-\pi)\left[\bar{\lambda}^{N, 1}(1-\psi)\left(1-\bar{\lambda}^{N, 2}\right)\right]}=0 .
$$

Then the expected continuation payoff the citizens will get after they choose not to revolt is

$$
V^{Z}\left(\alpha=0 \mid \widehat{\delta}=\Delta^{1}, \widehat{\rho}=0\right)=\mu\left(C \mid \widehat{\delta}=\Delta^{1}\right)\left(\Delta^{1}+\Delta^{2}\right)+\left[1-\mu\left(C \mid \widehat{\delta}=\Delta^{1}\right)\right] \psi \Delta^{2}=\psi \Delta^{2}
$$

Thus sequential rationality implies that the citizens will choose to revolt in $\left(\widehat{\delta}=\Delta^{1}, \widehat{\rho}=0\right)$ if and only if

$$
\begin{gathered}
V^{Z}\left(\alpha=1 \mid \widehat{\delta}=\Delta^{1}, \widehat{\rho}=0\right) \geq V^{Z}\left(\alpha=0 \mid \widehat{\delta}=\Delta^{1}, \widehat{\rho}=0\right) \\
\Leftrightarrow \alpha\left(\widehat{\delta}=\Delta^{1}, \widehat{\rho}=0\right)=1 \Leftrightarrow X-(\zeta)^{-1} \geq \psi \Delta^{2} \Leftrightarrow(\zeta)^{-1} \leq X-\psi \Delta^{2} .
\end{gathered}
$$


4. Information set $\left(\widehat{\delta}=\Delta^{1}, \widehat{\rho}=1\right)$ In this information set, the incumbent leader is removed from office by the selectorate; therefore, there is a new leader and thus the expected utility the citizens will get after they choose not to revolt does not depend on the previous observation on $\widehat{\delta}$. Then, the expected utility the citizens will get after they choose not to revolt is:

$$
V^{Z}\left(\alpha=0 \mid \widehat{\delta}=\Delta^{1}, \widehat{\rho}=1\right)=\pi\left(\Delta^{1}+\Delta^{2}\right)+(1-\pi) \psi \Delta^{2} .
$$

Sequential rationality implies that the citizens will choose to revolt in $\left(\widehat{\delta}=\Delta^{1}, \widehat{\rho}=1\right)$ if and only if

$$
\begin{gathered}
V^{Z}\left(\alpha=1 \mid \widehat{\delta}=\Delta^{1}, \widehat{\rho}=1\right) \geq V^{Z}\left(\alpha=0 \mid \widehat{\delta}=\Delta^{1}, \widehat{\rho}=1\right) \\
\Leftrightarrow \alpha\left(\widehat{\delta}=\Delta^{1}, \widehat{\rho}=1\right)=1 \Leftrightarrow(\zeta)^{-1} \leq X-\left[\pi\left(\Delta^{1}+\Delta^{2}\right)+(1-\pi) \psi \Delta^{2}\right] .
\end{gathered}
$$

5. Information set $\left(\widehat{\delta}=\Delta^{2}, \widehat{\rho}=0\right)$ In this information set the players' beliefs are

$$
\begin{gathered}
\mu\left(C \mid \widehat{\delta}=\Delta^{2}\right)= \\
=\frac{\pi\left[\left(1-\bar{\lambda}^{C, 1}\right) \bar{\lambda}^{C, 2}+\left(1-\bar{\lambda}^{C, 1}\right) \psi\left(1-\bar{\lambda}^{C, 2}\right)\right]}{\pi\left[\left(1-\bar{\lambda}^{C, 1}\right) \bar{\lambda}^{C, 2}+\left(1-\bar{\lambda}^{C, 1}\right) \psi\left(1-\bar{\lambda}^{C, 2}\right)\right]+(1-\pi)\left[\left(1-\bar{\lambda}^{N, 1}\right) \bar{\lambda}^{N, 2}+\left(1-\bar{\lambda}^{N, 1}\right) \psi\left(1-\bar{\lambda}^{N, 2}\right)\right]} \in[0,1]=c
\end{gathered}
$$
and the expected continuation payoff the citizens will get after they choose not to revolt is

$$
V^{Z}\left(\alpha=0 \mid \widehat{\delta}=\Delta^{2}, \widehat{\rho}=0\right)=\mu\left(C \mid \widehat{\delta}=\Delta^{2}\right)\left(\Delta^{1}+\Delta^{2}\right)+\left[1-\mu\left(C \mid \widehat{\delta}=\Delta^{2}\right)\right] \psi \Delta^{2}=\psi \Delta^{2}
$$

Thus sequential rationality implies that the citizens will choose to revolt in $\left(\widehat{\delta}=\Delta^{2}, \widehat{\rho}=1\right)$ if and only if

$$
\begin{gathered}
V^{Z}\left(\alpha=1 \mid \widehat{\delta}=\Delta^{2}, \widehat{\rho}=0\right) \geq V^{Z}\left(\alpha=0 \mid \widehat{\delta}=\Delta^{2}, \widehat{\rho}=0\right) \\
\Leftrightarrow \alpha\left(\widehat{\delta}=\Delta^{2}, \widehat{\rho}=0\right)=1 \Leftrightarrow X-(\zeta)^{-1} \geq \psi \Delta^{2} \Leftrightarrow(\zeta)^{-1} \leq X-\psi \Delta^{2}
\end{gathered}
$$

6. Information set $\left(\widehat{\delta}=\Delta^{2}, \widehat{\rho}=1\right)$ In this information set, the incumbent leader is removed from office by the selectorate; therefore, there is a new leader and thus the expected utility the citizens will get after they choose not to revolt does not depend on the previous observation on $\widehat{\delta}$. Then, the expected utility the citizens will get after they choose not to revolt is:

$$
V^{Z}\left(\alpha=0 \mid \widehat{\delta}=\Delta^{2}, \widehat{\rho}=1\right)=\pi\left(\Delta^{1}+\Delta^{2}\right)+(1-\pi) \psi \Delta^{2} .
$$

Sequential rationality implies that the citizens will choose to revolt in $\left(\widehat{\delta}=\Delta^{1}, \widehat{\rho}=1\right)$ if and only if

$$
\begin{gathered}
V^{Z}\left(\alpha=0 \mid \widehat{\delta}=\Delta^{2}, \widehat{\rho}=1\right) \geq V^{Z}\left(\alpha=0 \mid \widehat{\delta}=\Delta^{2}, \widehat{\rho}=1\right) \\
\Leftrightarrow \alpha\left(\widehat{\delta}=\Delta^{2}, \widehat{\rho}=1\right)=1 \Leftrightarrow(\zeta)^{-1} \leq X-\left[\pi\left(\Delta^{1}+\Delta^{2}\right)+(1-\pi) \psi \Delta^{2}\right] .
\end{gathered}
$$


7. Information set $(\widehat{\delta}=0, \widehat{\rho}=0)$ In this information set the players' beliefs are $\mu(C \mid \widehat{\delta}=0)=\frac{\pi\left[\left(1-\bar{\lambda}^{C, 1}\right)(1-\psi)\left(1-\bar{\lambda}^{C, 2}\right)\right]}{\pi\left[\left(1-\bar{\lambda}^{C, 1}\right)(1-\psi)\left(1-\bar{\lambda}^{C, 2}\right)\right]+(1-\pi)\left[\left(1-\bar{\lambda}^{N, 1}\right)(1-\psi)\left(1-\bar{\lambda}^{N, 2}\right)\right]} \in[0,1]=0$ and the expected continuation payoff the citizens will get after they choose not to revolt is

$$
V^{Z}(\alpha=0 \mid \widehat{\delta}=0, \widehat{\rho}=0)=\mu(C \mid \widehat{\delta}=0)\left(\Delta^{1}+\Delta^{2}\right)+[1-\mu(C \mid \widehat{\delta}=0)] \psi \Delta^{2}=\psi \Delta^{2} .
$$

Thus sequential rationality implies that the citizens will choose to revolt in $\left(\widehat{\delta}=\Delta^{1}, \widehat{\rho}=0\right)$ if and only if

$$
\begin{gathered}
V^{Z}(\alpha=1 \mid \widehat{\delta}=0, \widehat{\rho}=0) \geq V^{Z}(\alpha=0 \mid \widehat{\delta}=0, \widehat{\rho}=0) \\
\Leftrightarrow \alpha(\widehat{\delta}=0, \widehat{\rho}=0)=1 \Leftrightarrow X-(\zeta)^{-1} \geq \psi \Delta^{2} \Leftrightarrow(\zeta)^{-1} \leq X-\psi \Delta^{2} .
\end{gathered}
$$

8. Information set $(\widehat{\delta}=0, \widehat{\rho}=1)$ In this information set, the incumbent leader is removed from office by the selectorate; therefore, there is a new leader and thus the expected utility the citizens will get after they choose not to revolt does not depend on the previous observation on $\widehat{\delta}$. Then, the expected utility the citizens will get after they choose not to revolt is:

$$
V^{Z}(\alpha=0 \mid \widehat{\delta}=0, \widehat{\rho}=1)=\pi\left(\Delta^{1}+\Delta^{2}\right)+(1-\pi) \psi \Delta^{2} .
$$

Sequential rationality implies that the citizens will choose to revolt in $\left(\widehat{\delta}=\Delta^{1}, \widehat{\rho}=1\right)$ if and only if

$$
\begin{gathered}
V^{Z}(\alpha=0 \mid \widehat{\delta}=0, \widehat{\rho}=1) \geq V^{Z}(\alpha=0 \mid \widehat{\delta}=0, \widehat{\rho}=1) \\
\Leftrightarrow \alpha(\widehat{\delta}=0, \widehat{\rho}=1)=1 \Leftrightarrow(\zeta)^{-1} \leq X-\left[\pi\left(\Delta^{1}+\Delta^{2}\right)+(1-\pi) \psi \Delta^{2}\right] .
\end{gathered}
$$

The Selectorate After knowing their first-period utility at the end of the first period, the selectorate chooses between coup $(\rho=1)$ or not $(\rho=0)$. This means that to derive the selectorate's sequential rational behavior, we should consider four possible information sets, where in each information set, there are two decision nodes depending on the type of leader. Let $V^{S}\left(\rho=1, \alpha^{B R} \mid \widehat{\delta}\right)$ be the expected continuation payoff for the selectorate in $\widehat{\delta}$ if he subverts the incumbent leader and the citizens will subsequently choose according to $\alpha^{B R}$. Note that in this case, the payoff does not depend on $\widehat{\delta}$ being either 0 or $\Delta$, since the leader has been changed. Therefore, for any $\widehat{\delta} \in\left\{0, \Delta^{1}, \Delta^{2}, \Delta^{1}+\Delta^{2}\right\}$

$V^{S}\left(\rho=1, \alpha^{B R} \mid \widehat{\delta}\right)=\left(1-\alpha^{B R}\right)\left[\pi \times\left(\Delta^{1}+\Delta^{2}\right)+(1-\pi) \times \psi \Delta^{2}+\phi \times \frac{X}{\phi}+(1-\phi) \times 0\right]+\alpha^{B R} \times 0=$ 


$$
=\left(1-\alpha^{B R}\right)\left[\pi\left(\Delta^{1}+\Delta^{2}\right)+(1-\pi) \psi \Delta^{2}+X\right]:
$$

as the type of the newly picked up leader is unknown, she will produce $\Delta^{1}+\Delta^{2}$ with probability $\pi$ and $\psi \Delta^{2}$ (in expected terms) otherwise. Moreover, both types of leader will distribute the entire social revenue to the selectorate, but the members of the selectorate who ousted the leader, with probability $\phi$, will be included in the successor's selectorate getting the patronage $\frac{X}{\phi}$ in the second period.

On the other hand

$$
V^{S}\left(\rho=0, \alpha^{B R} \mid \widehat{\delta}\right)=\left(1-\alpha^{B R}\right)\left[\mu(C \mid \widehat{\delta}, \rho=1)\left(\Delta^{1}+\Delta^{2}\right)+[1-\mu(C \mid \widehat{\delta}, \rho=1)] \psi \Delta^{2}+\frac{X}{\phi}\right] .
$$

Let consider the four regions in the space $\zeta \in[0,1]$ :

1. $\zeta \in\left[\frac{1}{X-\left[\pi^{+}\left(\Delta^{1}+\Delta^{2}\right)+\left(1-\pi^{+}\right) \psi \Delta^{2}\right]}, 1\right]$ then, considering the four selectorate's information sets:

(a) in $\left(\widehat{\delta}=\Delta^{1}+\Delta^{2}\right)$ the selectorate's beliefs are

$$
\begin{gathered}
\mu\left(C \mid \widehat{\delta}=\Delta^{1}+\Delta^{2}\right)= \\
=\frac{\pi\left[\bar{\lambda}^{C, 1} \bar{\lambda}^{C, 2}+\bar{\lambda}^{C, 1} \psi\left(1-\bar{\lambda}^{C, 2}\right)\right]}{\pi\left[\bar{\lambda}^{C, 1} \bar{\lambda}^{C, 2}+\lambda \psi\left(1-\bar{\lambda}^{C, 2}\right)\right]+(1-\pi)\left[\bar{\lambda}^{N, 1} \bar{\lambda}^{N, 2}+\bar{\lambda}^{N, 1} \psi\left(1-\bar{\lambda}^{N, 2}\right)\right]}=\frac{\pi}{\pi+(1-\pi) \psi} \equiv \pi \\
\rho\left(\alpha^{B R} \mid \widehat{\delta}=\Delta^{1}+\Delta^{2}\right)=0 \Leftrightarrow V^{S}\left(\rho=0, \alpha^{B R} \mid \widehat{\delta}=\Delta^{1}+\Delta^{2}\right) \geq V^{S}\left(\rho=1, \alpha^{B R} \mid \widehat{\delta}=\Delta^{1}+\Delta^{2}\right) \Leftrightarrow \\
\Leftrightarrow\left(1-\alpha^{B R}\right)\left[\pi^{+}\left(\Delta^{1}+\Delta^{2}\right)+\left(1-\pi^{+}\right) \psi \Delta^{2}+\frac{X}{\phi}\right] \geq\left(1-\alpha^{B R}\right)\left[\pi\left(\Delta^{1}+\Delta^{2}\right)+(1-\pi) \psi \Delta^{2}+X\right] \Leftrightarrow \\
\Leftrightarrow \rho\left(\widehat{\delta}=\Delta^{1}+\Delta^{2} \mid \alpha^{B R}\right)^{B R} \in[0,1]
\end{gathered}
$$

(b) in $\left(\widehat{\delta}=\Delta^{1}\right)$ the selectorate's beliefs are

$$
\begin{gathered}
\mu\left(C \mid \widehat{\delta}=\Delta^{1}\right)=\frac{\pi\left[\bar{\lambda}^{C, 1}(1-\psi)\left(1-\bar{\lambda}^{C, 2}\right)\right]}{\pi\left[\bar{\lambda}^{C, 1}(1-\psi)\left(1-\bar{\lambda}^{C, 2}\right)\right]+(1-\pi)\left[\bar{\lambda}^{N, 1}(1-\psi)\left(1-\bar{\lambda}^{N, 2}\right)\right]}=0 \\
\rho\left(\alpha^{B R} \mid \widehat{\delta}=\Delta^{1}\right)=0 \Leftrightarrow V^{S}\left(\rho=0, \alpha^{B R} \mid \widehat{\delta}=\Delta^{1}\right) \geq V^{S}\left(\rho=1, \alpha^{B R} \mid \widehat{\delta}=\Delta^{1}\right) \Leftrightarrow \\
\Leftrightarrow\left(1-\alpha^{B R}\right)\left[\psi \Delta^{2}+\frac{X}{\phi}\right] \geq\left(1-\alpha^{B R}\right)\left[\pi\left(\Delta^{1}+\Delta^{2}\right)+(1-\pi) \psi \Delta^{2}+X\right] \Leftrightarrow 0 \geq 0 \Leftrightarrow \\
\Leftrightarrow \rho\left(\widehat{\delta}=\Delta^{1} \mid \alpha^{B R}\right)^{B R} \in[0,1]
\end{gathered}
$$


(c) in $\left(\widehat{\delta}=\Delta^{2}\right)$ the selectorate's beliefs are

$$
\begin{gathered}
\mu\left(C \mid \widehat{\delta}=\Delta^{2}\right)= \\
=\frac{\pi\left[\left(1-\bar{\lambda}^{C, 1}\right) \bar{\lambda}^{C, 2}+\left(1-\bar{\lambda}^{C, 1}\right) \psi\left(1-\bar{\lambda}^{C, 2}\right)\right]}{\pi\left[\left(1-\bar{\lambda}^{C, 1}\right) \bar{\lambda}^{C, 2}+\left(1-\bar{\lambda}^{C, 1}\right) \psi\left(1-\bar{\lambda}^{C, 2}\right)\right]+(1-\pi)\left[\left(1-\bar{\lambda}^{N, 1}\right) \bar{\lambda}^{N, 2}+\left(1-\bar{\lambda}^{N, 1}\right) \psi\left(1-\bar{\lambda}^{N,}\right.\right.} \\
\in[0,1]=0 \\
\rho\left(\alpha^{B R} \mid \widehat{\delta}=\Delta^{2}\right)=0 \Leftrightarrow V^{S}\left(\rho=0, \alpha^{B R} \mid \widehat{\delta}=\Delta^{2}\right) \geq V^{S}\left(\rho=1, \alpha^{B R} \mid \widehat{\delta}=\Delta^{2}\right) \Leftrightarrow \\
\Leftrightarrow\left(1-\alpha^{B R}\right)\left[\psi \Delta^{2}+\frac{X}{\phi}\right] \geq\left(1-\alpha^{B R}\right)\left[\pi\left(\Delta^{1}+\Delta^{2}\right)+(1-\pi) \psi \Delta^{2}+X\right] \Leftrightarrow 0 \geq 0 \Leftrightarrow \\
\Leftrightarrow \rho\left(\widehat{\delta}=\Delta^{2} \mid \alpha^{B R}\right)^{B R} \in[0,1]
\end{gathered}
$$

(d) in $(\widehat{\delta}=0)$ the selectorate's beliefs are

$$
\begin{gathered}
\mu(C \mid \widehat{\delta}=0)=\frac{\pi\left[\left(1-\bar{\lambda}^{C, 1}\right)(1-\psi)\left(1-\bar{\lambda}^{C, 2}\right)\right]}{\pi\left[\left(1-\bar{\lambda}^{C, 1}\right)(1-\psi)\left(1-\bar{\lambda}^{C, 2}\right)\right]+(1-\pi)\left[\left(1-\bar{\lambda}^{N, 1}\right)(1-\psi)\left(1-\bar{\lambda}^{N, 2}\right)\right]} \in[0,1]=0 \\
\rho\left(\alpha^{B R} \mid \widehat{\delta}=0\right)=1 \Leftrightarrow V^{S}\left(\rho=1, \alpha^{B R} \mid \widehat{\delta}=0\right) \geq V^{S}\left(\rho=0, \alpha^{B R} \mid \widehat{\delta}=0\right) \Leftrightarrow \\
\Leftrightarrow\left(1-\alpha^{B R}\right)\left[\psi \Delta^{2}+\frac{X}{\phi}\right] \geq\left(1-\alpha^{B R}\right)\left[\pi\left(\Delta^{1}+\Delta^{2}\right)+(1-\pi) \psi \Delta^{2}+X\right] \Leftrightarrow 0 \geq 0 \Leftrightarrow \\
\Leftrightarrow \rho\left(\widehat{\delta}=0 \mid \alpha^{B R}\right)^{B R} \in[0,1]
\end{gathered}
$$

2. $\zeta \in\left[\frac{1}{X-\left[\pi\left(\Delta^{1}+\Delta^{2}\right)+(1-\pi) \psi \Delta^{2}\right]}, \frac{1}{X-\left[\pi^{+}\left(\Delta^{1}+\Delta^{2}\right)+\left(1-\pi^{+}\right) \psi \Delta^{2}\right]}\right]$ then, considering the four selectorate's information sets:

(a) in $\left(\widehat{\delta}=\Delta^{1}+\Delta^{2}\right)$ the selectorate's beliefs are

$$
\begin{aligned}
& \mu(C \mid \widehat{\delta}\left.=\Delta^{1}+\Delta^{2}\right)= \\
&=\frac{\pi\left[\bar{\lambda}^{C, 1} \bar{\lambda}^{C, 2}+\bar{\lambda}^{C, 1} \psi\left(1-\bar{\lambda}^{C, 2}\right)\right]}{\pi\left[\bar{\lambda}^{C, 1} \bar{\lambda}^{C, 2}+\lambda \psi\left(1-\bar{\lambda}^{C, 2}\right)\right]+(1-\pi)\left[\bar{\lambda}^{N, 1} \bar{\lambda}^{N, 2}+\bar{\lambda}^{N, 1} \psi\left(1-\bar{\lambda}^{N, 2}\right)\right]}=\frac{\pi}{\pi+(1-\pi) \psi} \equiv \pi^{-} \\
& \rho\left(\alpha^{B R} \mid \widehat{\delta}=\Delta^{1}+\Delta^{2}\right)=0 \Leftrightarrow V^{S}\left(\rho=0, \alpha^{B R} \mid \widehat{\delta}=\Delta^{1}+\Delta^{2}\right) \geq V^{S}\left(\rho=1, \alpha^{B R} \mid \widehat{\delta}=\Delta^{1}+\Delta^{2}\right) \Leftrightarrow \\
& \Leftrightarrow \pi^{+}\left(\Delta^{1}+\Delta^{2}\right)+\left(1-\pi^{+}\right) \psi \Delta^{2}+\frac{X}{\phi} \geq 0 \Leftrightarrow \rho\left(\widehat{\delta}=\Delta^{1}+\Delta^{2} \mid \alpha^{B R}\right)^{B R}=0
\end{aligned}
$$


(b) in $\left(\widehat{\delta}=\Delta^{1}\right)$ the selectorate's beliefs are

$$
\begin{gathered}
\mu\left(C \mid \widehat{\delta}=\Delta^{1}\right)=\frac{\pi\left[\bar{\lambda}^{C, 1}(1-\psi)\left(1-\bar{\lambda}^{C, 2}\right)\right]}{\pi\left[\bar{\lambda}^{C, 1}(1-\psi)\left(1-\bar{\lambda}^{C, 2}\right)\right]+(1-\pi)\left[\bar{\lambda}^{N, 1}(1-\psi)\left(1-\bar{\lambda}^{N, 2}\right)\right]}=0 \\
\rho\left(\alpha^{B R} \mid \widehat{\delta}=\Delta^{1}\right)=0 \Leftrightarrow V^{S}\left(\rho=0, \alpha^{B R} \mid \widehat{\delta}=\Delta^{1}\right) \geq V^{S}\left(\rho=1, \alpha^{B R} \mid \widehat{\delta}=\Delta^{1}\right) \Leftrightarrow \\
\Leftrightarrow\left(1-\alpha^{B R}\right)\left[\psi \Delta^{2}+\frac{X}{\phi}\right] \geq\left(1-\alpha^{B R}\right)\left[\pi\left(\Delta^{1}+\Delta^{2}\right)+(1-\pi) \psi \Delta^{2}+X\right] \Leftrightarrow \\
\Leftrightarrow \rho\left(\widehat{\delta}=\Delta^{1} \mid \alpha^{B R}\right)^{B R} \in[0,1]
\end{gathered}
$$

(c) in $\left(\widehat{\delta}=\Delta^{2}\right)$ the selectorate's beliefs are

$$
\begin{gathered}
\mu\left(C \mid \widehat{\delta}=\Delta^{2}\right)= \\
=\frac{\pi\left[\left(1-\bar{\lambda}^{C, 1}\right) \bar{\lambda}^{C, 2}+\left(1-\bar{\lambda}^{C, 1}\right) \psi\left(1-\bar{\lambda}^{C, 2}\right)\right]}{\pi\left[\left(1-\bar{\lambda}^{C, 1}\right) \bar{\lambda}^{C, 2}+\left(1-\bar{\lambda}^{C, 1}\right) \psi\left(1-\bar{\lambda}^{C, 2}\right)\right]+(1-\pi)\left[\left(1-\bar{\lambda}^{N, 1}\right) \bar{\lambda}^{N, 2}+\left(1-\bar{\lambda}^{N, 1}\right) \psi\left(1-\bar{\lambda}^{N,}\right.\right.} \\
\in[0,1]=0 \\
\rho\left(\alpha^{B R} \mid \widehat{\delta}=\Delta^{2}\right)=0 \Leftrightarrow V^{S}\left(\rho=0, \alpha^{B R} \mid \widehat{\delta}=\Delta^{2}\right) \geq V^{S}\left(\rho=1, \alpha^{B R} \mid \widehat{\delta}=\Delta^{2}\right) \Leftrightarrow \\
\Leftrightarrow\left(1-\alpha^{B R}\right)\left[\psi \Delta^{2}+\frac{X}{\phi}\right] \geq\left(1-\alpha^{B R}\right)\left[\pi\left(\Delta^{1}+\Delta^{2}\right)+(1-\pi) \psi \Delta^{2}+X\right] \Leftrightarrow \\
\Leftrightarrow \rho\left(\widehat{\delta}=\Delta^{2} \mid \alpha^{B R}\right)^{B R} \in[0,1]
\end{gathered}
$$

(d) in $(\widehat{\delta}=0)$ the selectorate's beliefs are

$$
\begin{gathered}
\mu(C \mid \widehat{\delta}=0)=\frac{\pi\left[\left(1-\bar{\lambda}^{C, 1}\right)(1-\psi)\left(1-\bar{\lambda}^{C, 2}\right)\right]}{\pi\left[\left(1-\bar{\lambda}^{C, 1}\right)(1-\psi)\left(1-\bar{\lambda}^{C, 2}\right)\right]+(1-\pi)\left[\left(1-\bar{\lambda}^{N, 1}\right)(1-\psi)\left(1-\bar{\lambda}^{N, 2}\right)\right]} \in[0,1]=0 \\
\rho\left(\alpha^{B R} \widehat{\delta}=0\right)=0 \Leftrightarrow V^{S}\left(\rho=0, \alpha^{B R} \mid \widehat{\delta}=0\right) \geq V^{S}\left(\rho=1, \alpha^{B R} \mid \widehat{\delta}=0\right) \Leftrightarrow \\
\Leftrightarrow\left(1-\alpha^{B R}\right)\left[\psi \Delta^{2}+\frac{X}{\phi}\right] \geq\left(1-\alpha^{B R}\right)\left[\pi\left(\Delta^{1}+\Delta^{2}\right)+(1-\pi) \psi \Delta^{2}+X\right] \Leftrightarrow \\
\Leftrightarrow \rho\left(\widehat{\delta}=0 \mid \alpha^{B R}\right)^{B R} \in[0,1]
\end{gathered}
$$

3. $\zeta \in\left[\frac{1}{X-\psi \Delta^{2}}, \frac{1}{X-\left[\pi\left(\Delta^{1}+\Delta^{2}\right)+(1-\pi) \psi \Delta^{2}\right]}\right]$ then, considering the four selectorate's information sets: 
(a) in $\left(\widehat{\delta}=\Delta^{1}+\Delta^{2}\right)$ the selectorate's beliefs are

$$
\begin{gathered}
\mu\left(C \mid \widehat{\delta}=\Delta^{1}+\Delta^{2}\right)=\frac{\pi\left[\bar{\lambda}^{C, 1} \bar{\lambda}^{C, 2}+\bar{\lambda}^{C, 1} \psi\left(1-\bar{\lambda}^{C, 2}\right)\right]}{\pi\left[\bar{\lambda}^{C, 1} \bar{\lambda}^{C, 2}+\lambda \psi\left(1-\bar{\lambda}^{C, 2}\right)\right]+(1-\pi)\left[\bar{\lambda}^{N, 1} \bar{\lambda}^{N, 2}+\bar{\lambda}^{N, 1} \psi\left(1-\bar{\lambda}^{N, 2}\right)\right]}= \\
=\frac{\pi}{\pi+(1-\pi) \psi} \equiv \pi^{+} \\
\rho\left(\alpha^{B R} \mid \widehat{\delta}=\Delta^{1}+\Delta^{2}\right)=0 \Leftrightarrow V^{S}\left(\rho=0, \alpha^{B R} \mid \widehat{\delta}=\Delta^{1}+\Delta^{2}\right) \geq V^{S}\left(\rho=1, \alpha^{B R} \mid \widehat{\delta}=\Delta^{1}+\Delta^{2}\right) \Leftrightarrow \\
\Leftrightarrow \pi^{+}\left(\Delta^{1}+\Delta^{2}\right)+\left(1-\pi^{+}\right) \psi \Delta^{2}+\frac{X}{\phi} \geq \pi\left(\Delta^{1}+\Delta^{2}\right)+(1-\pi) \psi \Delta^{2}+X \Leftrightarrow \\
\Leftrightarrow \rho\left(\widehat{\delta}=\Delta^{1}+\Delta^{2} \mid \alpha^{B R}\right)^{B R}=0
\end{gathered}
$$

(b) in $\left(\widehat{\delta}=\Delta^{1}\right)$ the selectorate's beliefs are

$$
\begin{gathered}
\mu\left(C \mid \widehat{\delta}=\Delta^{1}\right)=\frac{\pi\left[\bar{\lambda}^{C, 1}(1-\psi)\left(1-\bar{\lambda}^{C, 2}\right)\right]}{\pi\left[\bar{\lambda}^{C, 1}(1-\psi)\left(1-\bar{\lambda}^{C, 2}\right)\right]+(1-\pi)\left[\bar{\lambda}^{N, 1}(1-\psi)\left(1-\bar{\lambda}^{N, 2}\right)\right]}=0 \\
\rho\left(\alpha^{B R} \mid \widehat{\delta}=\Delta^{1}\right)=0 \Leftrightarrow V^{S}\left(\rho=0, \alpha^{B R} \mid \widehat{\delta}=\Delta^{1}\right) \geq V^{S}\left(\rho=1, \alpha^{B R} \mid \widehat{\delta}=\Delta^{1}\right) \Leftrightarrow \\
\Leftrightarrow 0 \geq \pi\left(\Delta^{1}+\Delta^{2}\right)+(1-\pi) \psi \Delta^{2}+X \Leftrightarrow \\
\Leftrightarrow \rho\left(\widehat{\delta}=\Delta^{1} \mid \alpha^{B R}\right)^{B R}=1
\end{gathered}
$$

(c) in $\left(\widehat{\delta}=\Delta^{2}\right)$ the selectorate's beliefs are

$$
\begin{gathered}
\mu\left(C \mid \widehat{\delta}=\Delta^{2}\right)= \\
=\frac{\pi\left[\left(1-\bar{\lambda}^{C, 1}\right) \bar{\lambda}^{C, 2}+\left(1-\bar{\lambda}^{C, 1}\right) \psi\left(1-\bar{\lambda}^{C, 2}\right)\right]}{\pi\left[\left(1-\bar{\lambda}^{C, 1}\right) \bar{\lambda}^{C, 2}+\left(1-\bar{\lambda}^{C, 1}\right) \psi\left(1-\bar{\lambda}^{C, 2}\right)\right]+(1-\pi)\left[\left(1-\bar{\lambda}^{N, 1}\right) \bar{\lambda}^{N, 2}+\left(1-\bar{\lambda}^{N, 1}\right) \psi\left(1-\bar{\lambda}^{N,}\right.\right.} \\
\in[0,1]=0 \\
\rho\left(\alpha^{B R} \mid \widehat{\delta}=\Delta^{2}\right)=0 \Leftrightarrow V^{S}\left(\rho=0, \alpha^{B R} \mid \widehat{\delta}=\Delta^{2}\right) \geq V^{S}\left(\rho=1, \alpha^{B R} \mid \widehat{\delta}=\Delta^{2}\right) \Leftrightarrow \\
\Leftrightarrow 0 \geq \pi\left(\Delta^{1}+\Delta^{2}\right)+(1-\pi) \psi \Delta^{2}+X \Leftrightarrow \rho\left(\widehat{\delta}=\Delta^{2} \mid \alpha^{B R}\right)^{B R}=1
\end{gathered}
$$

(d) in $(\widehat{\delta}=0)$ the selectorate's beliefs are

$$
\begin{gathered}
\mu(C \mid \widehat{\delta}=0)=\frac{\pi\left[\left(1-\bar{\lambda}^{C, 1}\right)(1-\psi)\left(1-\bar{\lambda}^{C, 2}\right)\right]}{\pi\left[\left(1-\bar{\lambda}^{C, 1}\right)(1-\psi)\left(1-\bar{\lambda}^{C, 2}\right)\right]+(1-\pi)\left[\left(1-\bar{\lambda}^{N, 1}\right)(1-\psi)\left(1-\bar{\lambda}^{N, 2}\right)\right]} \in[0,1]=0 \\
\rho\left(\alpha^{B R} \mid \widehat{\delta}=0\right)=0 \Leftrightarrow V^{S}\left(\rho=0, \alpha^{B R} \mid \widehat{\delta}=0\right) \geq V^{S}\left(\rho=1, \alpha^{B R} \mid \widehat{\delta}=0\right) \Leftrightarrow \\
\Leftrightarrow 0 \geq \pi\left(\Delta^{1}+\Delta^{2}\right)+(1-\pi) \psi \Delta^{2}+X \Leftrightarrow \rho\left(\widehat{\delta}=0 \mid \alpha^{B R}\right)^{B R}=1
\end{gathered}
$$


4. $\zeta \in\left[0, \frac{1}{X-\psi \Delta^{2}}\right]$ then, considering the four selectorate's information sets:

(a) in $\left(\widehat{\delta}=\Delta^{1}+\Delta^{2}\right)$ the selectorate's beliefs are

$$
\begin{gathered}
\mu\left(C \mid \widehat{\delta}=\Delta^{1}+\Delta^{2}\right)=\frac{\pi\left[\bar{\lambda}^{C, 1} \bar{\lambda}^{C, 2}+\bar{\lambda}^{C, 1} \psi\left(1-\bar{\lambda}^{C, 2}\right)\right]}{\pi\left[\bar{\lambda}^{C, 1} \bar{\lambda}^{C, 2}+\lambda \psi\left(1-\bar{\lambda}^{C, 2}\right)\right]+(1-\pi)\left[\bar{\lambda}^{N, 1} \bar{\lambda}^{N, 2}+\bar{\lambda}^{N, 1} \psi\left(1-\bar{\lambda}^{N, 2}\right)\right]}= \\
=\frac{\pi}{\pi+(1-\pi) \psi} \equiv \pi^{+} \\
\rho\left(\alpha^{B R} \mid \widehat{\delta}=\Delta^{1}+\Delta^{2}\right)=0 \Leftrightarrow V^{S}\left(\rho=0, \alpha^{B R} \mid \widehat{\delta}=\Delta^{1}+\Delta^{2}\right) \geq V^{S}\left(\rho=1, \alpha^{B R} \mid \widehat{\delta}=\Delta^{1}+\Delta^{2}\right) \Leftrightarrow \\
\Leftrightarrow \pi^{+}\left(\Delta^{1}+\Delta^{2}\right)+\left(1-\pi^{+}\right) \psi \Delta^{2}+\frac{X}{\phi} \geq \pi\left(\Delta^{1}+\Delta^{2}\right)+(1-\pi) \psi \Delta^{2}+X \Leftrightarrow \\
\Leftrightarrow \rho\left(\widehat{\delta}=\Delta^{1}+\Delta^{2} \mid \alpha^{B R}\right)^{B R}=0
\end{gathered}
$$

(b) in $\left(\widehat{\delta}=\Delta^{1}\right)$ the selectorate's beliefs are

$$
\begin{gathered}
\mu\left(C \mid \widehat{\delta}=\Delta^{1}\right)=\frac{\pi\left[\bar{\lambda}^{C, 1}(1-\psi)\left(1-\bar{\lambda}^{C, 2}\right)\right]}{\pi\left[\bar{\lambda}^{C, 1}(1-\psi)\left(1-\bar{\lambda}^{C, 2}\right)\right]+(1-\pi)\left[\bar{\lambda}^{N, 1}(1-\psi)\left(1-\bar{\lambda}^{N, 2}\right)\right]}=0 \\
\rho\left(\alpha^{B R} \mid \widehat{\delta}=\Delta^{1}\right)=0 \Leftrightarrow V^{S}\left(\rho=0, \alpha^{B R} \mid \widehat{\delta}=\Delta^{1}\right) \geq V^{S}\left(\rho=1, \alpha^{B R} \mid \widehat{\delta}=\Delta^{1}\right) \Leftrightarrow \\
\Leftrightarrow \psi \Delta^{2}+\frac{X}{\phi} \geq \pi\left(\Delta^{1}+\Delta^{2}\right)+(1-\pi) \psi \Delta^{2}+X \Leftrightarrow \phi \leq \frac{X}{\pi\left(\Delta^{1}+\Delta^{2}-\psi \Delta^{2}\right)+X} \Leftrightarrow \\
\Leftrightarrow \rho\left(\widehat{\delta}=\Delta^{1} \mid \alpha^{B R}\right)^{B R}=\left\{\begin{array}{lll}
0 & \text { if } \phi \in\left[0, \frac{X}{\pi\left(\Delta^{1}+\Delta^{2}-\psi \Delta^{2}\right)+X}\right] \\
1 & \text { if } & \phi \in\left[\frac{X}{\pi\left(\Delta^{1}+\Delta^{2}-\psi \Delta^{2}\right)+X}, 1\right]
\end{array}\right.
\end{gathered}
$$

(c) in $\left(\widehat{\delta}=\Delta^{2}\right)$ the selectorate's beliefs are

$$
\begin{gathered}
\mu\left(C \mid \widehat{\delta}=\Delta^{2}\right)= \\
=\frac{\pi\left[\left(1-\bar{\lambda}^{C, 1}\right) \bar{\lambda}^{C, 2}+\left(1-\bar{\lambda}^{C, 1}\right) \psi\left(1-\bar{\lambda}^{C, 2}\right)\right]}{\pi\left[\left(1-\bar{\lambda}^{C, 1}\right) \bar{\lambda}^{C, 2}+\left(1-\bar{\lambda}^{C, 1}\right) \psi\left(1-\bar{\lambda}^{C, 2}\right)\right]+(1-\pi)\left[\left(1-\bar{\lambda}^{N, 1}\right) \bar{\lambda}^{N, 2}+\left(1-\bar{\lambda}^{N, 1}\right) \psi\left(1-\bar{\lambda}^{N,},\right.\right.} \\
\in[0,1]=0 \\
\rho\left(\alpha^{B R} \mid \widehat{\delta}=\Delta^{2}\right)=0 \Leftrightarrow V^{S}\left(\rho=0, \alpha^{B R} \mid \widehat{\delta}=\Delta^{2}\right) \geq V^{S}\left(\rho=1, \alpha^{B R} \mid \widehat{\delta}=\Delta^{2}\right) \Leftrightarrow \\
\Leftrightarrow \frac{X}{\phi} \geq \pi\left(\Delta^{1}+\Delta^{2}\right)-\pi \psi \Delta^{2}+X \Leftrightarrow \phi \leq \frac{X}{\pi\left(\Delta^{1}+\Delta^{2}-\psi \Delta^{2}\right)+X} \Leftrightarrow \\
\Leftrightarrow \rho\left(\widehat{\delta}=\Delta^{2} \mid \alpha^{B R}\right)^{B R}=\left\{\begin{array}{ccc}
0 & \text { if } & \phi \in\left[0, \frac{X}{\pi\left(\Delta^{1}+\Delta^{2}-\psi \Delta^{2}\right)+X}\right] \\
1 & \text { if } & \phi \in\left[\frac{X}{\pi\left(\Delta^{1}+\Delta^{2}-\psi \Delta^{2}\right)+X}, 1\right]
\end{array}\right.
\end{gathered}
$$


(d) in $(\widehat{\delta}=0)$ the selectorate's beliefs are

$$
\begin{gathered}
\mu(C \mid \widehat{\delta}=0)=\frac{\pi\left[\left(1-\bar{\lambda}^{C, 1}\right)(1-\psi)\left(1-\bar{\lambda}^{C, 2}\right)\right]}{\pi\left[\left(1-\bar{\lambda}^{C, 1}\right)(1-\psi)\left(1-\bar{\lambda}^{C, 2}\right)\right]+(1-\pi)\left[\left(1-\bar{\lambda}^{N, 1}\right)(1-\psi)\left(1-\bar{\lambda}^{N, 2}\right)\right]} \in[0,1]=0 \\
\rho\left(\alpha^{B R} \mid \widehat{\delta}=0\right)=0 \Leftrightarrow V^{S}\left(\rho=0, \alpha^{B R} \mid \widehat{\delta}=0\right) \geq V^{S}\left(\rho=1, \alpha^{B R} \mid \widehat{\delta}=0\right) \Leftrightarrow \\
\Leftrightarrow \frac{X}{\phi} \geq \pi\left(\Delta^{1}+\Delta^{2}\right)-\pi \psi \Delta^{2}+X \Leftrightarrow \phi \leq \frac{X}{\pi\left(\Delta^{1}+\Delta^{2}-\psi \Delta^{2}\right)+X} \Leftrightarrow \\
\Leftrightarrow \rho\left(\widehat{\delta}=0 \mid \alpha^{B R}\right)^{B R}=\left\{\begin{array}{lll}
0 & \text { if } & \phi \in\left[0, \frac{X}{\pi\left(\Delta^{1}+\Delta^{2}-\psi \Delta^{2}\right)+X}\right] \\
1 & \text { if } & \phi \in\left[\frac{X}{\pi\left(\Delta^{1}+\Delta^{2}-\psi \Delta^{2}\right)+X}, 1\right]
\end{array}\right.
\end{gathered}
$$

The Non Congruent Leader Now, we have to check whether the strategy profile $\left(\bar{\lambda}^{C, 1}, \bar{\lambda}^{C, 2}, \bar{\lambda}^{N, 1}, \bar{\lambda}^{N, 2}\right)=$ $(1,1,1,0)$ is part of of a SE. Her payoff will depend on $\rho^{B R}$ and $\alpha^{B R}$, hence on the basis of the previous $\alpha(\widehat{\delta}, \widehat{\rho})^{B R}$ and $\rho\left(\widehat{\delta} \mid \alpha^{B R}\right)^{B R}$ we need to distinguish five regions in the space $(\zeta, \phi) \in[0,1] \times[0,1]:$

1. if $(\zeta, \phi) \in\left[\frac{1}{X-\left[\pi^{+}\left(\Delta^{1}+\Delta^{2}\right)+\left(1-\pi^{+}\right) \psi \Delta^{2}\right]}, 1\right] \times[0,1]$ then

$$
V^{N}\left(1,1,1,0 ; \rho^{B R}, \alpha^{B R}\right)=\Delta^{1}+\psi \Delta^{2}+r_{1}^{2}+\frac{X}{\phi}+0
$$

while deviating she get

$$
V^{N}\left(\bar{\lambda}^{C, 1}, \bar{\lambda}^{C, 2}, \bar{\lambda}^{N, 1}, \bar{\lambda}^{N, 2} ; \rho^{B R}, \alpha^{B R}\right)= \begin{cases}\psi \Delta^{2}+r_{1}^{1}+r_{1}^{2}+\frac{X}{\phi}+0 & \text { if } \bar{\lambda}^{C, 1}, \bar{\lambda}^{C, 2}, \bar{\lambda}^{N, 1}, \bar{\lambda}^{N, 2}=1,1,0,0 \\ \Delta^{2}+r_{1}^{1}+\frac{X}{\phi}+0 & \text { if } \bar{\lambda}^{C, 1}, \bar{\lambda}^{C, 2}, \bar{\lambda}^{N, 1}, \bar{\lambda}^{N, 2}=1,1,0,1 \\ \Delta^{2}+\Delta^{1}+\frac{X}{\phi}+0 & \text { if } \bar{\lambda}^{C, 1}, \bar{\lambda}^{C, 2}, \bar{\lambda}^{N, 1}, \bar{\lambda}^{N, 2}=1,1,1,1\end{cases}
$$

which is always greater for $\left(\bar{\lambda}^{N, 1}, \bar{\lambda}^{N, 2}\right)=(0,0)$, hence in this region this is not part of an equilibrium;

2. $(\zeta, \phi) \in\left[\frac{1}{X-\left[\pi\left(\Delta^{1}+\Delta^{2}\right)+(1-\pi) \psi \Delta^{2}\right]}, \frac{1}{X-\left[\pi^{+}\left(\Delta^{1}+\Delta^{2}\right)+\left(1-\pi^{+}\right) \psi \Delta^{2}\right]}\right] \times[0,1]$ thus

$$
V^{N}\left(1,1,1,0 ; \rho^{B R}, \alpha^{B R}\right)=\psi \Delta^{2}+\Delta^{1}+r_{1}^{2}+\frac{X}{\phi}+\psi\left[\psi \Delta^{2}+E\left(r_{2}^{1}\right)+E\left(r_{2}^{2}\right)+\frac{X}{\phi}\right]
$$

while deviating she get

$$
\begin{gathered}
V^{N}\left(\bar{\lambda}^{C, 1}, \bar{\lambda}^{C, 2}, \bar{\lambda}^{N, 1}, \bar{\lambda}^{N, 2} ; \rho^{B R}, \alpha^{B R}\right)= \\
= \begin{cases}\psi \Delta^{2}+r_{1}^{1}+r_{1}^{2}+\frac{X}{\phi}+0 & \text { if } \bar{\lambda}^{C, 1}, \bar{\lambda}^{C, 2}, \bar{\lambda}^{N, 1}, \bar{\lambda}^{N, 2}=1,1,0,0 \\
\Delta^{2}+r_{1}^{1}+\frac{X}{\phi}+0 & \text { if } \bar{\lambda}^{C, 1}, \bar{\lambda}^{C, 2}, \bar{\lambda}^{N, 1}, \bar{\lambda}^{N, 2}=1,1,0,1 \\
\Delta^{2}+\Delta^{1}+\frac{X}{\phi}+\psi \Delta^{2}+E\left(r_{2}^{1}\right)+E\left(r_{2}^{2}\right)+\frac{X}{\phi} & \text { if } \bar{\lambda}^{C, 1}, \bar{\lambda}^{C, 2}, \bar{\lambda}^{N, 1}, \bar{\lambda}^{N, 2}=1,1,1,1 .\end{cases}
\end{gathered}
$$


Then, the non congruent leader has no incentives to deviate if and only if

$$
\begin{gathered}
\left\{\begin{array}{l}
\psi \Delta^{2}+r_{1}^{1}+r_{1}^{2}+\frac{X}{\phi} \leq \psi \Delta^{2}+\Delta^{1}+r_{1}^{2}+\frac{X}{\phi}+\psi\left[\psi \Delta^{2}+E\left(r_{2}^{1}\right)+E\left(r_{2}^{2}\right)+\frac{X}{\phi}\right] \\
\Delta^{2}+\Delta^{1}+\frac{X}{\phi}+\psi \Delta^{2}+E\left(r_{2}^{1}\right)+E\left(r_{2}^{2}\right)+\frac{X}{\phi} \leq \psi \Delta^{2}+\Delta^{1}+r_{1}^{2}+\frac{X}{\phi}+\psi\left[\psi \Delta^{2}+E\left(r_{2}^{1}\right)+E\left(r_{2}^{2}\right)+\frac{X}{\phi}\right]
\end{array}\right. \\
\Leftrightarrow\left\{\begin{array}{l}
r_{1}^{1} \leq \Delta^{1}+\psi\left[\psi \Delta^{2}+E\left(r_{2}^{1}\right)+E\left(r_{2}^{2}\right)+\frac{X}{\phi}\right] \\
(1-\psi) \Delta^{2}+(1-\psi)\left[\psi \Delta^{2}+E\left(r_{2}^{1}\right)+E\left(r_{2}^{2}\right)+\frac{X}{\phi}\right] \leq r_{1}^{2}
\end{array}\right.
\end{gathered}
$$

Hence in this region the leader might choose

$$
\left(\bar{\lambda}^{C, 1}, \bar{\lambda}^{C, 2}, \bar{\lambda}^{N, 1}, \bar{\lambda}^{N, 2}\right)=(1,1,1,0)
$$

with probability

$$
\begin{aligned}
& \mathbf{P}\left\{r_{1}^{1} \leq \Delta^{1}+\psi\left[\psi \Delta^{2}+E\left(r_{2}^{1}\right)+E\left(r_{2}^{2}\right)+\frac{X}{\phi}\right] \& r_{1}^{2} \geq(1-\psi) \Delta^{2}+(1-\psi)\left[\psi \Delta^{2}+E\left(r_{2}^{1}\right)+E\left(r_{2}^{2}\right)+\frac{X}{\phi}\right]\right\}= \\
& =G_{1}\left(\Delta^{1}+\psi\left[\psi \Delta^{2}+E\left(r_{2}^{1}\right)+E\left(r_{2}^{2}\right)+\frac{X}{\phi}\right]\right)\left[1-G_{2}\left((1-\psi)\left[\Delta^{2}+\psi \Delta^{2}+E\left(r_{2}^{1}\right)+E\left(r_{2}^{2}\right)+\frac{X}{\phi}\right]\right)\right]
\end{aligned}
$$

however before to conclude we need to consider players' incentives with the other leader's strategies.

3. $(\zeta, \phi) \in\left[\frac{1}{X-\psi \Delta^{2}}, \frac{1}{X-\left[\pi\left(\Delta^{1}+\Delta^{2}\right)+(1-\pi) \psi \Delta^{2}\right]}\right] \times[0,1]$ thus

$$
V^{N}\left(1,1,1,0 ; \rho^{B R}, \alpha^{B R}\right)=\psi \Delta^{2}+\Delta^{1}+r_{1}^{2}+\frac{X}{\phi}+\psi\left[\psi \Delta^{2}+E\left(r_{2}^{1}\right)+E\left(r_{2}^{2}\right)+\frac{X}{\phi}\right]
$$

while deviating she get

$$
\begin{gathered}
V^{N}\left(\bar{\lambda}^{C, 1}, \bar{\lambda}^{C, 2}, \bar{\lambda}^{N, 1}, \bar{\lambda}^{N, 2} ; \rho^{B R}, \alpha^{B R}\right)= \\
=\left\{\begin{array}{lr}
\psi \Delta^{2}+r_{1}^{1}+r_{1}^{2}+\frac{X}{\phi} & \text { if } \bar{\lambda}^{C, 1}, \bar{\lambda}^{C, 2}, \bar{\lambda}^{N, 1}, \bar{\lambda}^{N, 2}=1,1,0,0 \\
\Delta^{2}+r_{1}^{1}+\frac{X}{\phi} & \text { if } \bar{\lambda}^{C, 1}, \bar{\lambda}^{C, 2}, \bar{\lambda}^{N, 1}, \bar{\lambda}^{N, 2}=1,1,0,1 \\
\Delta^{2}+\Delta^{1}+\frac{X}{\phi}+\psi \Delta^{2}+E\left(r_{2}^{1}\right)+E\left(r_{2}^{2}\right)+\frac{X}{\phi} & \text { if } \bar{\lambda}^{C, 1}, \bar{\lambda}^{C, 2}, \bar{\lambda}^{N, 1}, \bar{\lambda}^{N, 2}=1,1,1,1 .
\end{array}\right.
\end{gathered}
$$

Then, the non congruent leader has no incentives to deviate if and only if

$$
\begin{gathered}
\left\{\begin{array}{l}
\psi \Delta^{2}+r_{1}^{1}+r_{1}^{2}+\frac{X}{\phi} \leq \psi \Delta^{2}+\Delta^{1}+r_{1}^{2}+\frac{X}{\phi}+\psi\left[\psi \Delta^{2}+E\left(r_{2}^{1}\right)+E\left(r_{2}^{2}\right)+\frac{X}{\phi}\right] \\
\Delta^{2}+\Delta^{1}+\frac{X}{\phi}+\psi \Delta^{2}+E\left(r_{2}^{1}\right)+E\left(r_{2}^{2}\right)+\frac{X}{\phi} \leq \psi \Delta^{2}+\Delta^{1}+r_{1}^{2}+\frac{X}{\phi}+\psi\left[\psi \Delta^{2}+E\left(r_{2}^{1}\right)+E\left(r_{2}^{2}\right)+\frac{X}{\phi}\right]
\end{array}\right. \\
\Leftrightarrow\left\{\begin{array}{l}
r_{1}^{1} \leq \Delta^{1}+\psi\left[\psi \Delta^{2}+E\left(r_{2}^{1}\right)+E\left(r_{2}^{2}\right)+\frac{X}{\phi}\right] \\
(1-\psi) \Delta^{2}+(1-\psi)\left[\psi \Delta^{2}+E\left(r_{2}^{1}\right)+E\left(r_{2}^{2}\right)+\frac{X}{\phi}\right] \leq r_{1}^{2}
\end{array}\right.
\end{gathered}
$$

Hence in this region the leader might choose

$$
\left(\bar{\lambda}^{C, 1}, \bar{\lambda}^{C, 2}, \bar{\lambda}^{N, 1}, \bar{\lambda}^{N, 2}\right)=(1,1,1,0)
$$


with probability

$$
\begin{aligned}
& \mathbf{P}\left\{r_{1}^{1} \leq \Delta^{1}+\psi\left[\psi \Delta^{2}+E\left(r_{2}^{1}\right)+E\left(r_{2}^{2}\right)+\frac{X}{\phi}\right] \& r_{1}^{2} \geq(1-\psi) \Delta^{2}+(1-\psi)\left[\psi \Delta^{2}+E\left(r_{2}^{1}\right)+E\left(r_{2}^{2}\right)+\frac{X}{\phi}\right]\right\}= \\
& =G_{1}\left(\Delta^{1}+\psi\left[\psi \Delta^{2}+E\left(r_{2}^{1}\right)+E\left(r_{2}^{2}\right)+\frac{X}{\phi}\right]\right)\left[1-G_{2}\left((1-\psi)\left[\Delta^{2}+\psi \Delta^{2}+E\left(r_{2}^{1}\right)+E\left(r_{2}^{2}\right)+\frac{X}{\phi}\right]\right)\right]
\end{aligned}
$$

however before to conclude we need to consider players' incentives with the other leader's strategies.

4. $(\zeta, \phi) \in\left[0, \frac{1}{X-\psi \Delta^{2}}\right] \times\left[0, \frac{X}{\pi\left(\Delta^{1}+\Delta^{2}-\psi \Delta^{2}\right)+X}\right]$ thus

$$
V^{N}\left(1,1,1,0 ; \rho^{B R}, \alpha^{B R}\right)=\psi \Delta^{2}+\Delta^{1}+r_{1}^{2}+\frac{X}{\phi}+\psi \Delta^{2}+E\left(r_{2}^{1}\right)+E\left(r_{2}^{2}\right)+\frac{X}{\phi}
$$

while deviating she get

$$
\begin{gathered}
V^{N}\left(\bar{\lambda}^{C, 1}, \bar{\lambda}^{C, 2}, \bar{\lambda}^{N, 1}, \bar{\lambda}^{N, 2} ; \rho^{B R}, \alpha^{B R}\right)= \\
= \begin{cases}\psi \Delta^{2}+r_{1}^{1}+r_{1}^{2}+\frac{X}{\phi}+\psi \Delta^{2}+E\left(r_{2}^{1}\right)+E\left(r_{2}^{2}\right)+\frac{X}{\phi} & \text { if } \bar{\lambda}^{C, 1}, \bar{\lambda}^{C, 2}, \bar{\lambda}^{N, 1}, \bar{\lambda}^{N, 2}=1,1,0,0 \\
\Delta^{2}+r_{1}^{1}+\frac{X}{\phi}+\psi \Delta^{2}+E\left(r_{2}^{1}\right)+E\left(r_{2}^{2}\right)+\frac{X}{\phi} & \text { if } \bar{\lambda}^{C, 1}, \bar{\lambda}^{C, 2}, \bar{\lambda}^{N, 1}, \bar{\lambda}^{N, 2}=1,1,0,1 \\
\Delta^{2}+\Delta^{1}+\frac{X}{\phi}+\psi \Delta^{2}+E\left(r_{2}^{1}\right)+E\left(r_{2}^{2}\right)+\frac{X}{\phi} & \text { if } \bar{\lambda}^{C, 1}, \bar{\lambda}^{C, 2}, \bar{\lambda}^{N, 1}, \bar{\lambda}^{N, 2}=1,1,1,1 .\end{cases}
\end{gathered}
$$

Then, the non congruent leader has an incentives to deviate to $\left(\bar{\lambda}^{N, 1}, \bar{\lambda}^{N, 2}\right)=(0,0)$, hence, it can't be part of an equilibrium.

5. $(\zeta, \phi) \in\left[0, \frac{1}{X-\psi \Delta^{2}}\right] \times\left[\frac{X}{\pi\left(\Delta^{1}+\Delta^{2}\right)+X}, 1\right]$ thus

$$
V^{N}\left(1,1,1,0 ; \rho^{B R}, \alpha^{B R}\right)=\psi \Delta^{2}+\Delta^{1}+r_{1}^{2}+\frac{X}{\phi}+\psi\left[\psi \Delta^{2}+E\left(r_{2}^{1}\right)+E\left(r_{2}^{2}\right)+\frac{X}{\phi}\right]
$$

while deviating she get

$$
\begin{gathered}
V^{N}\left(\bar{\lambda}^{C, 1}, \bar{\lambda}^{C, 2}, \bar{\lambda}^{N, 1}, \bar{\lambda}^{N, 2} ; \rho^{B R}, \alpha^{B R}\right)= \\
=\left\{\begin{array}{lr}
\psi \Delta^{2}+r_{1}^{1}+r_{1}^{2}+\frac{X}{\phi} & \text { if } \bar{\lambda}^{C, 1}, \bar{\lambda}^{C, 2}, \bar{\lambda}^{N, 1}, \bar{\lambda}^{N, 2}=1,1,0,0 \\
\Delta^{2}+r_{1}^{1}+\frac{X}{\phi} & \text { if } \bar{\lambda}^{C, 1}, \bar{\lambda}^{C, 2}, \bar{\lambda}^{N, 1}, \bar{\lambda}^{N, 2}=1,1,0,1 \\
\Delta^{2}+\Delta^{1}+\frac{X}{\phi}+\psi \Delta^{2}+E\left(r_{2}^{1}\right)+E\left(r_{2}^{2}\right)+\frac{X}{\phi} & \text { if } \bar{\lambda}^{C, 1}, \bar{\lambda}^{C, 2}, \bar{\lambda}^{N, 1}, \bar{\lambda}^{N, 2}=1,1,1,1 .
\end{array}\right.
\end{gathered}
$$

Then, the non congruent leader has no incentives to deviate if and only if

$$
\begin{gathered}
\left\{\begin{array}{l}
\psi \Delta^{2}+r_{1}^{1}+r_{1}^{2}+\frac{X}{\phi} \leq \psi \Delta^{2}+\Delta^{1}+r_{1}^{2}+\frac{X}{\phi}+\psi\left[\psi \Delta^{2}+E\left(r_{2}^{1}\right)+E\left(r_{2}^{2}\right)+\frac{X}{\phi}\right] \\
\Delta^{2}+\Delta^{1}+\frac{X}{\phi}+\psi \Delta^{2}+E\left(r_{2}^{1}\right)+E\left(r_{2}^{2}\right)+\frac{X}{\phi} \leq \psi \Delta^{2}+\Delta^{1}+r_{1}^{2}+\frac{X}{\phi}+\psi\left[\psi \Delta^{2}+E\left(r_{2}^{1}\right)+E\left(r_{2}^{2}\right)+\frac{X}{\phi}\right]
\end{array}\right. \\
\Leftrightarrow\left\{\begin{array}{l}
r_{1}^{1} \leq \Delta^{1}+\psi\left[\psi \Delta^{2}+E\left(r_{2}^{1}\right)+E\left(r_{2}^{2}\right)+\frac{X}{\phi}\right] \\
(1-\psi) \Delta^{2}+(1-\psi)\left[\psi \Delta^{2}+E\left(r_{2}^{1}\right)+E\left(r_{2}^{2}\right)+\frac{X}{\phi}\right] \leq r_{1}^{2}
\end{array}\right.
\end{gathered}
$$


Hence in this region we may get an equilibrium:

$$
\left(\bar{\lambda}^{C, 1}, \bar{\lambda}^{C, 2}, \bar{\lambda}^{N, 1}, \bar{\lambda}^{N, 2}\right)=(1,1,1,0)
$$

with probability

$$
\begin{aligned}
& \mathbf{P}\left\{r_{1}^{1} \leq \Delta^{1}+\psi\left[\psi \Delta^{2}+E\left(r_{2}^{1}\right)+E\left(r_{2}^{2}\right)+\frac{X}{\phi}\right] \& r_{1}^{2} \geq(1-\psi) \Delta^{2}+(1-\psi)\left[\psi \Delta^{2}+E\left(r_{2}^{1}\right)+E\left(r_{2}^{2}\right)+\frac{X}{\phi}\right]\right\}= \\
& =G_{1}\left(\Delta^{1}+\psi\left[\psi \Delta^{2}+E\left(r_{2}^{1}\right)+E\left(r_{2}^{2}\right)+\frac{X}{\phi}\right]\right)\left[1-G_{2}\left((1-\psi)\left[\Delta^{2}+\psi \Delta^{2}+E\left(r_{2}^{1}\right)+E\left(r_{2}^{2}\right)+\frac{X}{\phi}\right]\right)\right]
\end{aligned}
$$

however before to conclude we need to consider players' incentives with the other leader's strategies.

3. Strategy profile $\left(\bar{\lambda}^{C, 1}, \bar{\lambda}^{C, 2}, \bar{\lambda}^{N, 1}, \bar{\lambda}^{N, 2}\right)=(1,1,0,1)$

The Citizens After knowing their first-period utility and the selectorate's choice at the end of the first period, the citizens choose between revolt $(\alpha=1)$ or not $(\alpha=0)$. This means that to derive the citizens' sequential rational behavior, we should consider eight possible information sets, where in each information set, there are two decision nodes depending on the type of leader. Let $V^{Z}(\alpha \mid \delta, \rho)$ be the expected continuation payoff for the citizens when they choose $\alpha$ if $(\delta, \rho)$ has been observed. The expected continuation utility that the citizens will get after they choose to initiate a revolution in $(\delta, \rho)$ is:

$$
V^{Z}(\alpha=1 \mid \delta, \rho)=(1-\phi) \times \frac{X-(\zeta)^{-1}}{1-\phi}+\phi \times 0=X-(\zeta)^{-1}
$$

Clearly, this payoff does not depend on their beliefs about the leader's type and thus on $(\delta, \rho)$. On the other hand, if the citizens decide to accommodate, the continuation payoff will depend on their beliefs about the type of leader which, in turn, will depend on their information at the time of deciding. Therefore, to find the citizens' rational behavior, we need to consider eight possible information sets and the citizens' beliefs in these information sets.

1. Information set $\left(\widehat{\delta}=\Delta^{1}+\Delta^{2}, \widehat{\rho}=0\right)$ In this information set the players' beliefs are $\mu\left(C \mid \widehat{\delta}=\Delta^{1}+\Delta^{2}\right)=\frac{\pi\left[\bar{\lambda}^{C, 1} \bar{\lambda}^{C, 2}+\bar{\lambda}^{C, 1} \psi\left(1-\bar{\lambda}^{C, 2}\right)\right]}{\pi\left[\bar{\lambda}^{C, 1} \bar{\lambda}^{C, 2}+\lambda \psi\left(1-\bar{\lambda}^{C, 2}\right)\right]+(1-\pi)\left[\bar{\lambda}^{N, 1} \bar{\lambda}^{N, 2}+\bar{\lambda}^{N, 1} \psi\left(1-\bar{\lambda}^{N, 2}\right)\right]}=1$

The expected continuation payoff the citizens will get after they choose not to revolt is $V^{Z}\left(\alpha=0 \mid \widehat{\delta}=\Delta^{1}+\Delta^{2}, \widehat{\rho}=0\right)=\mu\left(C \mid \widehat{\delta}=\Delta^{1}+\Delta^{2}\right)\left(\Delta^{1}+\Delta^{2}\right)+\left[1-\mu\left(C \mid \widehat{\delta}=\Delta^{1}+\Delta^{2}\right)\right] \psi \Delta^{2}=\left(\Delta^{1}+\Delta^{2}\right)$ 
then sequential rationality implies that the citizens will choose to revolt in $\left(\widehat{\delta}=\Delta^{1}+\Delta^{2}, \widehat{\rho}=0\right)$ if and only if

$$
\begin{gathered}
V^{Z}\left(\alpha=1 \mid \widehat{\delta}=\Delta^{1}+\Delta^{2}, \widehat{\rho}=0\right) \geq V^{Z}\left(\alpha=0 \mid \widehat{\delta}=\Delta^{1}+\Delta^{2}, \widehat{\rho}=0\right) \Leftrightarrow \\
\Leftrightarrow \alpha\left(\widehat{\delta}=\Delta^{1}+\Delta^{2}, \widehat{\rho}=0\right)=1 \Leftrightarrow X-(\zeta)^{-1} \geq\left(\Delta^{1}+\Delta^{2}\right) \Leftrightarrow(\zeta)^{-1} \leq X-\left(\Delta^{1}+\Delta^{2}\right) .
\end{gathered}
$$

2. Information set $\left(\widehat{\delta}=\Delta^{1}+\Delta^{2}, \widehat{\rho}=1\right)$ In this information set, the incumbent leader is removed from office by the selectorate; therefore, there is a new leader and thus the expected utility the citizens will get after they choose not to revolt does not depend on the previous observation on $\delta$. Then, the expected utility the citizens will get after they choose not to revolt is:

$$
V^{Z}\left(\alpha=0 \mid \widehat{\delta}=\Delta^{1}+\Delta^{2}, \widehat{\rho}=1\right)=\pi\left(\Delta^{1}+\Delta^{2}\right)+(1-\pi) \psi \Delta^{2} .
$$

Sequential rationality implies that the citizens will choose to revolt in $\left(\widehat{\delta}=\Delta^{1}+\Delta^{2}, \widehat{\rho}=1\right)$ if and only if

$$
\begin{gathered}
V^{Z}\left(\alpha=1 \mid \widehat{\delta}=\Delta^{1}+\Delta^{2}, \widehat{\rho}=1\right) \geq V^{Z}\left(\alpha=0 \mid \widehat{\delta}=\Delta^{1}+\Delta^{2}, \widehat{\rho}=1\right) \Leftrightarrow \\
\Leftrightarrow \alpha\left(\widehat{\delta}=\Delta^{1}+\Delta^{2}, \widehat{\rho}=1\right)=1 \Leftrightarrow(\zeta)^{-1} \leq X-\left[\pi\left(\Delta^{1}+\Delta^{2}\right)+(1-\pi) \psi \Delta^{2}\right] .
\end{gathered}
$$

3. Information set $\left(\widehat{\delta}=\Delta^{1}, \widehat{\rho}=0\right)$ In this information set the players' beliefs are

$$
\mu\left(C \mid \widehat{\delta}=\Delta^{1}\right)=\frac{\pi\left[\bar{\lambda}^{C, 1}(1-\psi)\left(1-\bar{\lambda}^{C, 2}\right)\right]}{\pi\left[\bar{\lambda}^{C, 1}(1-\psi)\left(1-\bar{\lambda}^{C, 2}\right)\right]+(1-\pi)\left[\bar{\lambda}^{N, 1}(1-\psi)\left(1-\bar{\lambda}^{N, 2}\right)\right]} \in[0,1]=0
$$

and the expected continuation payoff the citizens will get after they choose not to revolt is

$$
V^{Z}\left(\alpha=0 \mid \widehat{\delta}=\Delta^{1}, \widehat{\rho}=0\right)=\mu\left(C \mid \widehat{\delta}=\Delta^{1}\right)\left(\Delta^{1}+\Delta^{2}\right)+\left[1-\mu\left(C \mid \widehat{\delta}=\Delta^{1}\right)\right] \psi \Delta^{2}=\psi \Delta^{2}
$$

Thus sequential rationality implies that the citizens will choose to revolt in $\left(\widehat{\delta}=\Delta^{1}, \widehat{\rho}=0\right)$ if and only if

$$
\begin{gathered}
V^{Z}\left(\alpha=1 \mid \widehat{\delta}=\Delta^{1}, \widehat{\rho}=0\right) \geq V^{Z}\left(\alpha=0 \mid \widehat{\delta}=\Delta^{1}, \widehat{\rho}=0\right) \Leftrightarrow \\
\Leftrightarrow \alpha\left(\widehat{\delta}=\Delta^{1}, \widehat{\rho}=0\right)=1 \Leftrightarrow X-(\zeta)^{-1} \geq \psi \Delta^{2} \Leftrightarrow(\zeta)^{-1} \leq X-\psi \Delta^{2} .
\end{gathered}
$$


4. Information set $\left(\widehat{\delta}=\Delta^{1}, \widehat{\rho}=1\right)$ In this information set, the incumbent leader is removed from office by the selectorate; therefore, there is a new leader and thus the expected utility the citizens will get after they choose not to revolt does not depend on the previous observation on $\widehat{\delta}$. Then, the expected utility the citizens will get after they choose not to revolt is:

$$
V^{Z}\left(\alpha=0 \mid \widehat{\delta}=\Delta^{1}, \widehat{\rho}=1\right)=\pi\left(\Delta^{1}+\Delta^{2}\right)+(1-\pi) \psi \Delta^{2} .
$$

Sequential rationality implies that the citizens will choose to revolt in $\left(\widehat{\delta}=\Delta^{1}, \widehat{\rho}=1\right)$ if and only if

$$
\begin{gathered}
V^{Z}\left(\alpha=1 \mid \widehat{\delta}=\Delta^{1}, \widehat{\rho}=1\right) \geq V^{Z}\left(\alpha=0 \mid \widehat{\delta}=\Delta^{1}, \widehat{\rho}=1\right) \Leftrightarrow \\
\Leftrightarrow \alpha\left(\widehat{\delta}=\Delta^{1}, \widehat{\rho}=1\right)=1 \Leftrightarrow(\zeta)^{-1} \leq X-\left[\pi\left(\Delta^{1}+\Delta^{2}\right)+(1-\pi) \psi \Delta^{2}\right] .
\end{gathered}
$$

5. Information set $\left(\widehat{\delta}=\Delta^{2}, \widehat{\rho}=0\right)$ In this information set the players' beliefs are

$$
\begin{gathered}
\mu\left(C \mid \widehat{\delta}=\Delta^{2}\right)= \\
=\frac{\pi\left[\left(1-\bar{\lambda}^{C, 1}\right) \bar{\lambda}^{C, 2}+\left(1-\bar{\lambda}^{C, 1}\right) \psi\left(1-\bar{\lambda}^{C, 2}\right)\right]}{\pi\left[\left(1-\bar{\lambda}^{C, 1}\right) \bar{\lambda}^{C, 2}+\left(1-\bar{\lambda}^{C, 1}\right) \psi\left(1-\bar{\lambda}^{C, 2}\right)\right]+(1-\pi)\left[\left(1-\bar{\lambda}^{N, 1}\right) \bar{\lambda}^{N, 2}+\left(1-\bar{\lambda}^{N, 1}\right) \psi\left(1-\bar{\lambda}^{N, 2}\right)\right]}=0
\end{gathered}
$$

and the expected continuation payoff the citizens will get after they choose not to revolt is

$$
V^{Z}\left(\alpha=0 \mid \widehat{\delta}=\Delta^{2}, \widehat{\rho}=0\right)=\mu\left(C \mid \widehat{\delta}=\Delta^{2}\right)\left(\Delta^{1}+\Delta^{2}\right)+\left[1-\mu\left(C \mid \widehat{\delta}=\Delta^{2}\right)\right] \psi \Delta^{2}=\psi \Delta^{2} .
$$

Thus sequential rationality implies that the citizens will choose to revolt in $\left(\widehat{\delta}=\Delta^{2}, \widehat{\rho}=0\right)$ if and only if

$$
\begin{gathered}
V^{Z}\left(\alpha=1 \mid \widehat{\delta}=\Delta^{2}, \widehat{\rho}=0\right) \geq V^{Z}\left(\alpha=0 \mid \widehat{\delta}=\Delta^{2}, \widehat{\rho}=0\right) \Leftrightarrow \\
\Leftrightarrow \alpha\left(\widehat{\delta}=\Delta^{2}, \widehat{\rho}=0\right)=1 \Leftrightarrow X-(\zeta)^{-1} \geq \psi \Delta^{2} \Leftrightarrow(\zeta)^{-1} \leq X-\psi \Delta^{2} .
\end{gathered}
$$

6. Information set $\left(\widehat{\delta}=\Delta^{2}, \widehat{\rho}=1\right)$ In this information set, the incumbent leader is removed from office by the selectorate; therefore, there is a new leader and thus the expected utility the citizens will get after they choose not to revolt does not depend on the previous observation on $\delta$. Then, the expected utility the citizens will get after they choose not to revolt is:

$$
V^{Z}\left(\alpha=0 \mid \widehat{\delta}=\Delta^{2}, \widehat{\rho}=1\right)=\pi\left(\Delta^{1}+\Delta^{2}\right)+(1-\pi) \psi \Delta^{2} .
$$

Sequential rationality implies that the citizens will choose to revolt in $\left(\widehat{\delta}=\Delta^{1}, \widehat{\rho}=1\right)$ if and only if

$$
\begin{gathered}
V^{Z}\left(\alpha=0 \mid \widehat{\delta}=\Delta^{2}, \widehat{\rho}=1\right) \geq V^{Z}\left(\alpha=0 \mid \widehat{\delta}=\Delta^{2}, \widehat{\rho}=1\right) \Leftrightarrow \\
\Leftrightarrow \alpha\left(\widehat{\delta}=\Delta^{2}, \widehat{\rho}=1\right)=1 \Leftrightarrow(\zeta)^{-1} \leq X-\left[\pi\left(\Delta^{1}+\Delta^{2}\right)+(1-\pi) \psi \Delta^{2}\right] .
\end{gathered}
$$


7. Information set $(\widehat{\delta}=0, \widehat{\rho}=0)$ In this information set the players' beliefs are $\mu(C \mid \widehat{\delta}=0)=\frac{\pi\left[\left(1-\bar{\lambda}^{C, 1}\right)(1-\psi)\left(1-\bar{\lambda}^{C, 2}\right)\right]}{\pi\left[\left(1-\bar{\lambda}^{C, 1}\right)(1-\psi)\left(1-\bar{\lambda}^{C, 2}\right)\right]+(1-\pi)\left[\left(1-\bar{\lambda}^{N, 1}\right)(1-\psi)\left(1-\bar{\lambda}^{N, 2}\right)\right]} \in[0,1]$.

Then, using FI, we get

$$
\mu(C \mid \widehat{\delta}=0)=0
$$

and the expected continuation payoff the citizens will get after they choose not to revolt is

$$
V^{Z}(\alpha=0 \mid \widehat{\delta}=0, \widehat{\rho}=0)=\mu(C \mid \widehat{\delta}=0)\left(\Delta^{1}+\Delta^{2}\right)+[1-\mu(C \mid \widehat{\delta}=0)] \psi \Delta^{2}=\psi \Delta^{2}
$$

Thus sequential rationality implies that the citizens will choose to revolt in $\left(\widehat{\delta}=\Delta^{1}, \widehat{\rho}=1\right)$ if and only if

$$
\begin{gathered}
V^{Z}(\alpha=1 \mid \widehat{\delta}=0, \widehat{\rho}=0) \geq V^{Z}(\alpha=0 \mid \widehat{\delta}=0, \widehat{\rho}=0) \Leftrightarrow \\
\Leftrightarrow \alpha(\widehat{\delta}=0, \widehat{\rho}=0)=1 \Leftrightarrow X-(\zeta)^{-1} \geq \psi \Delta^{2} \Leftrightarrow(\zeta)^{-1} \leq X-\psi \Delta^{2} .
\end{gathered}
$$

8. Information set $(\widehat{\delta}=0, \widehat{\rho}=1)$ In this information set, the incumbent leader is removed from office by the selectorate; therefore, there is a new leader and thus the expected utility the citizens will get after they choose not to revolt does not depend on the previous observation on $\widehat{\delta}$. Then, the expected utility the citizens will get after they choose not to revolt is:

$$
V^{Z}(\alpha=0 \mid \widehat{\delta}=0, \widehat{\rho}=1)=\pi\left(\Delta^{1}+\Delta^{2}\right)+(1-\pi) \psi \Delta^{2} .
$$

Sequential rationality implies that the citizens will choose to revolt in $\left(\widehat{\delta}=\Delta^{1}, \widehat{\rho}=1\right)$ if and only if

$$
\begin{gathered}
V^{Z}(\alpha=0 \mid \widehat{\delta}=0, \widehat{\rho}=1) \geq V^{Z}(\alpha=0 \mid \widehat{\delta}=0, \widehat{\rho}=1) \Leftrightarrow \\
\Leftrightarrow \alpha(\widehat{\delta}=0, \widehat{\rho}=1)=1 \Leftrightarrow(\zeta)^{-1} \leq X-\left[\pi\left(\Delta^{1}+\Delta^{2}\right)+(1-\pi) \psi \Delta^{2}\right] .
\end{gathered}
$$

The Selectorate After knowing their first-period utility at the end of the first period, the selectorate chooses between coup $(\rho=1)$ or not $(\rho=0)$. This means that to derive the selectorate's sequential rational behavior, we should consider four possible information sets, where in each information set, there are two decision nodes depending on the type of leader. Let $V^{S}\left(\rho=1, \alpha^{B R} \mid \widehat{\delta}\right)$ be the expected continuation payoff for the selectorate in $\delta$ if he subverts the incumbent leader and the citizens will subsequently choose according to $\alpha^{B R}$. Note that in this 
case, the payoff does not depend on $\delta$ being either 0 or $\Delta^{1}$, or $\Delta^{2}$ or $\Delta^{1}+\Delta^{2}$, since the leader has been changed. Therefore

$$
\begin{gathered}
V^{S}\left(\rho=1, \alpha^{B R} \widehat{\delta}\right)=\left(1-\alpha^{B R}\right)\left[\pi \times\left(\Delta^{1}+\Delta^{2}\right)+(1-\pi) \times \psi \Delta^{2}+\phi \times \frac{X}{\phi}+(1-\phi) \times 0\right]+\alpha^{B R} \times 0= \\
=\left(1-\alpha^{B R}\right)\left[\pi\left(\Delta^{1}+\Delta^{2}\right)+(1-\pi) \psi \Delta^{2}+X\right]:
\end{gathered}
$$

as the type of the newly picked up leader is unknown, she will produce $\Delta^{1}+\Delta^{2}$ with probability $\pi$ and $\psi \Delta^{2}$ (in expected terms) otherwise. Moreover, both types of leader will distribute the entire social revenue to the selectorate, but the members of the selectorate who ousted the leader, with probability $\phi$, will be included in the successor's selectorate getting the patronage $\frac{X}{\phi}$ in the second period.

On the other hand

$$
V^{S}\left(\rho=0, \alpha^{B R} \mid \widehat{\delta}\right)=\left(1-\alpha^{B R}\right)\left[\mu(C \mid \widehat{\delta}, \rho=0)\left(\Delta^{1}+\Delta^{2}\right)+[1-\mu(C \mid \widehat{\delta}, \rho=0)] \psi \Delta^{2}+\frac{X}{\phi}\right] .
$$

Let consider the four regions in the space $\zeta \in[0,1]$ :

1. $\zeta \in\left[\frac{1}{X-\left(\Delta^{1}+\Delta^{2}\right)}, 1\right]$ then, considering the four selectorate's information sets:

(a) in $\left(\widehat{\delta}=\Delta^{1}+\Delta^{2}\right)$ the selectorate's beliefs are

$$
\begin{aligned}
& \mu\left(C \mid \widehat{\delta}=\Delta^{1}+\Delta^{2}\right)=\frac{\pi\left[\bar{\lambda}^{C, 1} \bar{\lambda}^{C, 2}+\bar{\lambda}^{C, 1} \psi\left(1-\bar{\lambda}^{C, 2}\right)\right]}{\pi\left[\bar{\lambda}^{C, 1} \bar{\lambda}^{C, 2}+\lambda \psi\left(1-\bar{\lambda}^{C, 2}\right)\right]+(1-\pi)\left[\bar{\lambda}^{N, 1} \bar{\lambda}^{N, 2}+\bar{\lambda}^{N, 1} \psi\left(1-\bar{\lambda}^{N, 2}\right)\right]}=1 \\
& \rho\left(\alpha^{B R} \mid \widehat{\delta}=\Delta^{1}+\Delta^{2}\right)=0 \Leftrightarrow V^{S}\left(\rho=0, \alpha^{B R} \mid \widehat{\delta}=\Delta^{1}+\Delta^{2}\right) \geq V^{S}\left(\rho=1, \alpha^{B R} \mid \widehat{\delta}=\Delta^{1}+\Delta^{2}\right) \Leftrightarrow \\
& \Leftrightarrow\left(1-\alpha^{B R}\right)\left[\left(\Delta^{1}+\Delta^{2}\right)+\frac{X}{\phi}\right] \geq\left(1-\alpha^{B R}\right)\left[\pi\left(\Delta^{1}+\Delta^{2}\right)+(1-\pi) \psi \Delta^{2}+X\right] \Leftrightarrow 0 \geq 0 \Leftrightarrow \\
& \Leftrightarrow \rho\left(\widehat{\delta}=\Delta^{1}+\Delta^{2} \mid \alpha^{B R}\right)^{B R} \in[0,1]
\end{aligned}
$$

(b) in $\left(\widehat{\delta}=\Delta^{1}\right)$ the selectorate's beliefs are

$$
\begin{gathered}
\mu\left(C \mid \widehat{\delta}=\Delta^{1}\right)=\frac{\pi\left[\bar{\lambda}^{C, 1}(1-\psi)\left(1-\bar{\lambda}^{C, 2}\right)\right]}{\pi\left[\bar{\lambda}^{C, 1}(1-\psi)\left(1-\bar{\lambda}^{C, 2}\right)\right]+(1-\pi)\left[\bar{\lambda}^{N, 1}(1-\psi)\left(1-\bar{\lambda}^{N, 2}\right)\right]} \in[0,1]=0 \\
\rho\left(\alpha^{B R} \mid \widehat{\delta}=\Delta^{1}\right)=0 \Leftrightarrow V^{S}\left(\rho=0, \alpha^{B R} \mid \widehat{\delta}=\Delta^{1}\right) \geq V^{S}\left(\rho=1, \alpha^{B R} \mid \widehat{\delta}=\Delta^{1}\right) \Leftrightarrow \\
\Leftrightarrow\left(1-\alpha^{B R}\right)\left[\psi \Delta^{2}+\frac{X}{\phi}\right] \geq\left(1-\alpha^{B R}\right)\left[\pi\left(\Delta^{1}+\Delta^{2}\right)+(1-\pi) \psi \Delta^{2}+X\right] \Leftrightarrow 0 \geq 0 \Leftrightarrow \\
\Leftrightarrow \rho\left(\widehat{\delta}=\Delta^{1} \mid \alpha^{B R}\right)^{B R} \in[0,1]
\end{gathered}
$$


(c) in $\left(\widehat{\delta}=\Delta^{2}\right)$ the selectorate's beliefs are

$$
\begin{gathered}
\mu\left(C \mid \widehat{\delta}=\Delta^{2}\right)= \\
=\frac{\pi\left[\left(1-\bar{\lambda}^{C, 1}\right) \bar{\lambda}^{C, 2}+\left(1-\bar{\lambda}^{C, 1}\right) \psi\left(1-\bar{\lambda}^{C, 2}\right)\right]}{\pi\left[\left(1-\bar{\lambda}^{C, 1}\right) \bar{\lambda}^{C, 2}+\left(1-\bar{\lambda}^{C, 1}\right) \psi\left(1-\bar{\lambda}^{C, 2}\right)\right]+(1-\pi)\left[\left(1-\bar{\lambda}^{N, 1}\right) \bar{\lambda}^{N, 2}+\left(1-\bar{\lambda}^{N, 1}\right) \psi\left(1-\bar{\lambda}^{N, 2}\right)\right]} \\
\rho\left(\alpha^{B R} \widehat{\delta}=\Delta^{2}\right)=0 \Leftrightarrow V^{S}\left(\rho=0, \alpha^{B R} \mid \widehat{\delta}=\Delta^{2}\right) \geq V^{S}\left(\rho=1, \alpha^{B R} \mid \widehat{\delta}=\Delta^{2}\right) \Leftrightarrow \\
\Leftrightarrow\left(1-\alpha^{B R}\right)\left[\psi \Delta^{2}+\frac{X}{\phi}\right] \geq\left(1-\alpha^{B R}\right)\left[\pi\left(\Delta^{1}+\Delta^{2}\right)+(1-\pi) \psi \Delta^{2}+X\right] \Leftrightarrow 0 \geq 0 \Leftrightarrow \\
\Leftrightarrow \rho\left(\widehat{\delta}=\Delta^{2} \mid \alpha^{B R}\right)^{B R} \in[0,1]
\end{gathered}
$$

(d) in $(\widehat{\delta}=0)$ the selectorate's beliefs are

$$
\begin{gathered}
\mu(C \mid \widehat{\delta}=0)=\frac{\pi\left[\left(1-\bar{\lambda}^{C, 1}\right)(1-\psi)\left(1-\bar{\lambda}^{C, 2}\right)\right]}{\pi\left[\left(1-\bar{\lambda}^{C, 1}\right)(1-\psi)\left(1-\bar{\lambda}^{C, 2}\right)\right]+(1-\pi)\left[\left(1-\bar{\lambda}^{N, 1}\right)(1-\psi)\left(1-\bar{\lambda}^{N, 2}\right)\right]} \in[0,1]=0 \\
\rho\left(\alpha^{B R} \mid \widehat{\delta}=0\right)=0 \Leftrightarrow V^{S}\left(\rho=0, \alpha^{B R} \mid \widehat{\delta}=0\right) \geq V^{S}\left(\rho=1, \alpha^{B R} \mid \widehat{\delta}=0\right) \Leftrightarrow \\
\Leftrightarrow\left(1-\alpha^{B R}\right)\left[\psi \Delta^{2}+\frac{X}{\phi}\right] \geq\left(1-\alpha^{B R}\right)\left[\pi\left(\Delta^{1}+\Delta^{2}\right)+(1-\pi) \psi \Delta^{2}+X\right] \Leftrightarrow 0 \geq 0 \Leftrightarrow \\
\Leftrightarrow \rho\left(\widehat{\delta}=0 \mid \alpha^{B R}\right)^{B R} \in[0,1]
\end{gathered}
$$

2. $\zeta \in\left[\frac{1}{X-\left[\pi\left(\Delta^{1}+\Delta^{2}\right)+(1-\pi) \psi \Delta^{2}\right]}, \frac{1}{X-\left(\Delta^{1}+\Delta^{2}\right)}\right]$ then, considering the four selectorate's information sets:

(a) in $\left(\widehat{\delta}=\Delta^{1}+\Delta^{2}\right)$ the selectorate's beliefs are

$$
\begin{aligned}
& \mu\left(C \mid \widehat{\delta}=\Delta^{1}+\Delta^{2}\right)=\frac{\pi\left[\bar{\lambda}^{C, 1} \bar{\lambda}^{C, 2}+\bar{\lambda}^{C, 1} \psi\left(1-\bar{\lambda}^{C, 2}\right)\right]}{\pi\left[\bar{\lambda}^{C, 1} \bar{\lambda}^{C, 2}+\lambda \psi\left(1-\bar{\lambda}^{C, 2}\right)\right]+(1-\pi)\left[\bar{\lambda}^{N, 1} \bar{\lambda}^{N, 2}+\bar{\lambda}^{N, 1} \psi\left(1-\bar{\lambda}^{N, 2}\right)\right]}=1 \\
& \rho\left(\alpha^{B R} \mid \widehat{\delta}=\Delta^{1}+\Delta^{2}\right)=0 \Leftrightarrow V^{S}\left(\rho=0, \alpha^{B R} \mid \widehat{\delta}=\Delta^{1}+\Delta^{2}\right) \geq V^{S}\left(\rho=1, \alpha^{B R} \mid \widehat{\delta}=\Delta^{1}+\Delta^{2}\right) \Leftrightarrow \\
& \Leftrightarrow\left(1-\alpha^{B R}\right)\left[\left(\Delta^{1}+\Delta^{2}\right)+\frac{X}{\phi}\right] \geq\left(1-\alpha^{B R}\right)\left[\pi\left(\Delta^{1}+\Delta^{2}\right)+(1-\pi) \psi \Delta^{2}+X\right] \Leftrightarrow\left(\Delta^{1}+\Delta^{2}\right)+\frac{X}{\phi} \geq 0 \Leftrightarrow \\
& \Leftrightarrow \rho\left(\widehat{\delta}=\Delta^{1}+\Delta^{2} \mid \alpha^{B R}\right)^{B R}=0
\end{aligned}
$$

(b) in $\left(\widehat{\delta}=\Delta^{1}\right)$ the selectorate's beliefs are

$$
\mu\left(C \mid \widehat{\delta}=\Delta^{1}\right)=\frac{\pi\left[\bar{\lambda}^{C, 1}(1-\psi)\left(1-\bar{\lambda}^{C, 2}\right)\right]}{\pi\left[\bar{\lambda}^{C, 1}(1-\psi)\left(1-\bar{\lambda}^{C, 2}\right)\right]+(1-\pi)\left[\bar{\lambda}^{N, 1}(1-\psi)\left(1-\bar{\lambda}^{N, 2}\right)\right]} \in[0,1]=0
$$




$$
\begin{gathered}
\rho\left(\alpha^{B R} \mid \widehat{\delta}=\Delta^{1}\right)=0 \Leftrightarrow V^{S}\left(\rho=0, \alpha^{B R} \mid \widehat{\delta}=\Delta^{1}\right) \geq V^{S}\left(\rho=1, \alpha^{B R} \mid \widehat{\delta}=\Delta^{1}\right) \Leftrightarrow \\
\Leftrightarrow\left(1-\alpha^{B R}\right)\left[\psi \Delta^{2}+\frac{X}{\phi}\right] \geq\left(1-\alpha^{B R}\right)\left[\pi\left(\Delta^{1}+\Delta^{2}\right)+(1-\pi) \psi \Delta^{2}+X\right] \Leftrightarrow 0 \geq 0 \Leftrightarrow \\
\Leftrightarrow \rho\left(\widehat{\delta}=\Delta^{1} \mid \alpha^{B R}\right)^{B R} \in[0,1]
\end{gathered}
$$

(c) in $\left(\widehat{\delta}=\Delta^{2}\right)$ the selectorate's beliefs are

$$
\begin{gathered}
\mu\left(C \mid \widehat{\delta}=\Delta^{2}\right)= \\
=\frac{\pi\left[\left(1-\bar{\lambda}^{C, 1}\right) \bar{\lambda}^{C, 2}+\left(1-\bar{\lambda}^{C, 1}\right) \psi\left(1-\bar{\lambda}^{C, 2}\right)\right]}{\pi\left[\left(1-\bar{\lambda}^{C, 1}\right) \bar{\lambda}^{C, 2}+\left(1-\bar{\lambda}^{C, 1}\right) \psi\left(1-\bar{\lambda}^{C, 2}\right)\right]+(1-\pi)\left[\left(1-\bar{\lambda}^{N, 1}\right) \bar{\lambda}^{N, 2}+\left(1-\bar{\lambda}^{N, 1}\right) \psi\left(1-\bar{\lambda}^{N, 2}\right)\right]} \\
\rho\left(\alpha^{B R} \mid \widehat{\delta}=\Delta^{2}\right)=0 \Leftrightarrow V^{S}\left(\rho=0, \alpha^{B R} \mid \widehat{\delta}=\Delta^{2}\right) \geq V^{S}\left(\rho=1, \alpha^{B R} \mid \widehat{\delta}=\Delta^{2}\right) \Leftrightarrow \\
\Leftrightarrow\left(1-\alpha^{B R}\right)\left[\psi \Delta^{2}+\frac{X}{\phi}\right] \geq\left(1-\alpha^{B R}\right)\left[\pi\left(\Delta^{1}+\Delta^{2}\right)+(1-\pi) \psi \Delta^{2}+X\right] \Leftrightarrow 0 \geq 0 \Leftrightarrow \\
\Leftrightarrow \rho\left(\widehat{\delta}=\Delta^{2} \mid \alpha^{B R}\right)^{B R} \in[0,1]
\end{gathered}
$$

(d) in $(\widehat{\delta}=0)$ the selectorate's beliefs are

$$
\begin{gathered}
\mu(C \mid \widehat{\delta}=0)=\frac{\pi\left[\left(1-\bar{\lambda}^{C, 1}\right)(1-\psi)\left(1-\bar{\lambda}^{C, 2}\right)\right]}{\pi\left[\left(1-\bar{\lambda}^{C, 1}\right)(1-\psi)\left(1-\bar{\lambda}^{C, 2}\right)\right]+(1-\pi)\left[\left(1-\bar{\lambda}^{N, 1}\right)(1-\psi)\left(1-\bar{\lambda}^{N, 2}\right)\right]} \in[0,1]=0 \\
\rho\left(\alpha^{B R} \mid \widehat{\delta}=0\right)=0 \Leftrightarrow V^{S}\left(\rho=0, \alpha^{B R} \mid \widehat{\delta}=0\right) \geq V^{S}\left(\rho=1, \alpha^{B R} \mid \widehat{\delta}=0\right) \Leftrightarrow \\
\Leftrightarrow\left(1-\alpha^{B R}\right)\left[\psi \Delta^{2}+\frac{X}{\phi}\right] \geq\left(1-\alpha^{B R}\right)\left[\pi\left(\Delta^{1}+\Delta^{2}\right)+(1-\pi) \psi \Delta^{2}+X\right] \Leftrightarrow 0 \geq 0 \Leftrightarrow \\
\Leftrightarrow \rho\left(\widehat{\delta}=0 \mid \alpha^{B R}\right)^{B R} \in[0,1]
\end{gathered}
$$

3. $\zeta \in\left[\frac{1}{X-\psi \Delta^{2}}, \frac{1}{X-\left[\pi\left(\Delta^{1}+\Delta^{2}\right)+(1-\pi) \psi \Delta^{2}\right]}\right]$ then, considering the four selectorate's information sets:

(a) in $\left(\widehat{\delta}=\Delta^{1}+\Delta^{2}\right)$ the selectorate's beliefs are

$$
\begin{gathered}
\mu\left(C \mid \widehat{\delta}=\Delta^{1}+\Delta^{2}\right)=\frac{\pi\left[\bar{\lambda}^{C, 1} \bar{\lambda}^{C, 2}+\bar{\lambda}^{C, 1} \psi\left(1-\bar{\lambda}^{C, 2}\right)\right]}{\pi\left[\bar{\lambda}^{C, 1} \bar{\lambda}^{C, 2}+\lambda \psi\left(1-\bar{\lambda}^{C, 2}\right)\right]+(1-\pi)\left[\bar{\lambda}^{N, 1} \bar{\lambda}^{N, 2}+\bar{\lambda}^{N, 1} \psi\left(1-\bar{\lambda}^{N, 2}\right)\right]}=1 \\
\rho\left(\alpha^{B R} \mid \widehat{\delta}=\Delta^{1}+\Delta^{2}\right)=0 \Leftrightarrow V^{S}\left(\rho=0, \alpha^{B R} \mid \widehat{\delta}=\Delta^{1}+\Delta^{2}\right) \geq V^{S}\left(\rho=1, \alpha^{B R} \mid \widehat{\delta}=\Delta^{1}+\Delta^{2}\right) \Leftrightarrow \\
\Leftrightarrow\left(\Delta^{1}+\Delta^{2}\right)+\frac{X}{\phi} \geq \pi\left(\Delta^{1}+\Delta^{2}\right)+(1-\pi) \psi \Delta^{2}+X \Leftrightarrow \\
\Leftrightarrow \rho\left(\widehat{\delta}=\Delta^{1}+\Delta^{2} \mid \alpha^{B R}\right)^{B R}=0
\end{gathered}
$$


(b) in $\left(\widehat{\delta}=\Delta^{1}\right)$ the selectorate's beliefs are

$$
\begin{gathered}
\mu\left(C \mid \widehat{\delta}=\Delta^{1}\right)=\frac{\pi\left[\bar{\lambda}^{C, 1}(1-\psi)\left(1-\bar{\lambda}^{C, 2}\right)\right]}{\pi\left[\bar{\lambda}^{C, 1}(1-\psi)\left(1-\bar{\lambda}^{C, 2}\right)\right]+(1-\pi)\left[\bar{\lambda}^{N, 1}(1-\psi)\left(1-\bar{\lambda}^{N, 2}\right)\right]} \in[0,1]=0 \\
\rho\left(\alpha^{B R} \mid \widehat{\delta}=\Delta^{1}\right)=0 \Leftrightarrow V^{S}\left(\rho=0, \alpha^{B R} \mid \widehat{\delta}=\Delta^{1}\right) \geq V^{S}\left(\rho=1, \alpha^{B R} \mid \widehat{\delta}=\Delta^{1}\right) \Leftrightarrow \\
\Leftrightarrow 0 \geq \pi\left(\Delta^{1}+\Delta^{2}\right)+(1-\pi) \psi \Delta^{2}+X \Leftrightarrow \\
\Leftrightarrow \rho\left(\widehat{\delta}=\Delta^{1} \mid \alpha^{B R}\right)^{B R}=0
\end{gathered}
$$

(c) in $\left(\widehat{\delta}=\Delta^{2}\right)$ the selectorate's beliefs are

$$
\begin{gathered}
\mu\left(C \mid \widehat{\delta}=\Delta^{2}\right)= \\
=\frac{\pi\left[\left(1-\bar{\lambda}^{C, 1}\right) \bar{\lambda}^{C, 2}+\left(1-\bar{\lambda}^{C, 1}\right) \psi\left(1-\bar{\lambda}^{C, 2}\right)\right]}{\pi\left[\left(1-\bar{\lambda}^{C, 1}\right) \bar{\lambda}^{C, 2}+\left(1-\bar{\lambda}^{C, 1}\right) \psi\left(1-\bar{\lambda}^{C, 2}\right)\right]+(1-\pi)\left[\left(1-\bar{\lambda}^{N, 1}\right) \bar{\lambda}^{N, 2}+\left(1-\bar{\lambda}^{N, 1}\right) \psi\left(1-\bar{\lambda}^{N, 2}\right)\right]} \\
\rho\left(\alpha^{B R} \mid \widehat{\delta}=\Delta^{2}\right)=0 \Leftrightarrow V^{S}\left(\rho=0, \alpha^{B R} \mid \widehat{\delta}=\Delta^{2}\right) \geq V^{S}\left(\rho=1, \alpha^{B R} \mid \widehat{\delta}=\Delta^{2}\right) \Leftrightarrow \\
\Leftrightarrow\left(1-\alpha^{B R}\right)\left[\psi \Delta^{2}+\frac{X}{\phi}\right] \geq\left(1-\alpha^{B R}\right)\left[\pi\left(\Delta^{1}+\Delta^{2}\right)+(1-\pi) \psi \Delta^{2}+X\right] \Leftrightarrow \\
\Leftrightarrow 0 \geq \pi\left(\Delta^{1}+\Delta^{2}\right)+(1-\pi) \psi \Delta^{2}+X \Leftrightarrow \rho\left(\widehat{\delta}=\Delta^{2} \mid \alpha^{B R}\right)^{B R}=1
\end{gathered}
$$

(d) in $(\widehat{\delta}=0)$ the selectorate's beliefs are

$$
\begin{gathered}
\mu(C \mid \widehat{\delta}=0)=\frac{\pi\left[\left(1-\bar{\lambda}^{C, 1}\right)(1-\psi)\left(1-\bar{\lambda}^{C, 2}\right)\right]}{\pi\left[\left(1-\bar{\lambda}^{C, 1}\right)(1-\psi)\left(1-\bar{\lambda}^{C, 2}\right)\right]+(1-\pi)\left[\left(1-\bar{\lambda}^{N, 1}\right)(1-\psi)\left(1-\bar{\lambda}^{N, 2}\right)\right]} \in[0,1]=0 \\
\rho\left(\alpha^{B R} \mid \widehat{\delta}=0\right)=0 \Leftrightarrow V^{S}\left(\rho=0, \alpha^{B R} \mid \widehat{\delta}=0\right) \geq V^{S}\left(\rho=1, \alpha^{B R} \mid \widehat{\delta}=0\right) \Leftrightarrow \\
\Leftrightarrow 0 \geq \pi\left(\Delta^{1}+\Delta^{2}\right)+(1-\pi) \psi \Delta^{2}+X \Leftrightarrow \\
\Leftrightarrow \rho\left(\widehat{\delta}=0 \mid \alpha^{B R}\right)^{B R}=1
\end{gathered}
$$

4. $\zeta \in\left[0, \frac{1}{X-\psi \Delta^{2}}\right]$ then, considering the four selectorate's information sets:

(a) in $\left(\widehat{\delta}=\Delta^{1}+\Delta^{2}\right)$ the selectorate's beliefs are

$$
\begin{aligned}
& \mu\left(C \mid \widehat{\delta}=\Delta^{1}+\Delta^{2}\right)=\frac{\pi\left[\bar{\lambda}^{C, 1} \bar{\lambda}^{C, 2}+\bar{\lambda}^{C, 1} \psi\left(1-\bar{\lambda}^{C, 2}\right)\right]}{\pi\left[\bar{\lambda}^{C, 1} \bar{\lambda}^{C, 2}+\lambda \psi\left(1-\bar{\lambda}^{C, 2}\right)\right]+(1-\pi)\left[\bar{\lambda}^{N, 1} \bar{\lambda}^{N, 2}+\bar{\lambda}^{N, 1} \psi\left(1-\bar{\lambda}^{N, 2}\right)\right]}=1 \\
& \rho\left(\alpha^{B R} \mid \widehat{\delta}=\Delta^{1}+\Delta^{2}\right)=0 \Leftrightarrow V^{S}\left(\rho=0, \alpha^{B R} \mid \widehat{\delta}=\Delta^{1}+\Delta^{2}\right) \geq V^{S}\left(\rho=1, \alpha^{B R} \mid \widehat{\delta}=\Delta^{1}+\Delta^{2}\right) \Leftrightarrow
\end{aligned}
$$




$$
\begin{gathered}
\Leftrightarrow\left(\Delta^{1}+\Delta^{2}\right)+\frac{X}{\phi} \geq \pi\left(\Delta^{1}+\Delta^{2}\right)+(1-\pi) \psi \Delta^{2}+X \Leftrightarrow \\
\Leftrightarrow \rho\left(\widehat{\delta}=\Delta^{1}+\Delta^{2} \mid \alpha^{B R}\right)^{B R}=0
\end{gathered}
$$

(b) in $\left(\widehat{\delta}=\Delta^{1}\right)$ the selectorate's beliefs are

$$
\begin{gathered}
\mu\left(C \mid \widehat{\delta}=\Delta^{1}\right)=\frac{\pi\left[\bar{\lambda}^{C, 1}(1-\psi)\left(1-\bar{\lambda}^{C, 2}\right)\right]}{\pi\left[\bar{\lambda}^{C, 1}(1-\psi)\left(1-\bar{\lambda}^{C, 2}\right)\right]+(1-\pi)\left[\bar{\lambda}^{N, 1}(1-\psi)\left(1-\bar{\lambda}^{N, 2}\right)\right]} \in[0,1]=0 \\
\rho\left(\alpha^{B R} \mid \widehat{\delta}=\Delta^{1}\right)=0 \Leftrightarrow V^{S}\left(\rho=0, \alpha^{B R} \mid \widehat{\delta}=\Delta^{1}\right) \geq V^{S}\left(\rho=1, \alpha^{B R} \mid \widehat{\delta}=\Delta^{1}\right) \Leftrightarrow \\
\Leftrightarrow \psi \Delta^{2}+\frac{X}{\phi} \geq \pi\left(\Delta^{1}+\Delta^{2}\right)+(1-\pi) \psi \Delta^{2}+X \Leftrightarrow \\
\Leftrightarrow \rho\left(\widehat{\delta}=\Delta^{1} \mid \alpha^{B R}\right)^{B R}=\left\{\begin{array}{lll}
0 & \text { if } & \phi \in\left[0, \frac{X}{\pi\left(\Delta^{1}+\Delta^{2}-\psi \Delta^{2}\right)+X}\right] \\
1 & \text { if } & \phi \in\left[\frac{X}{\pi\left(\Delta^{1}+\Delta^{2}-\psi \Delta^{2}\right)+X}, 1\right]
\end{array}\right.
\end{gathered}
$$

(c) in $\left(\widehat{\delta}=\Delta^{2}\right)$ the selectorate's beliefs are

$$
\begin{gathered}
\mu\left(C \mid \widehat{\delta}=\Delta^{2}\right)= \\
=\frac{\pi\left[\left(1-\bar{\lambda}^{C, 1}\right) \bar{\lambda}^{C, 2}+\left(1-\bar{\lambda}^{C, 1}\right) \psi\left(1-\bar{\lambda}^{C, 2}\right)\right]}{\pi\left[\left(1-\bar{\lambda}^{C, 1}\right) \bar{\lambda}^{C, 2}+\left(1-\bar{\lambda}^{C, 1}\right) \psi\left(1-\bar{\lambda}^{C, 2}\right)\right]+(1-\pi)\left[\left(1-\bar{\lambda}^{N, 1}\right) \bar{\lambda}^{N, 2}+\left(1-\bar{\lambda}^{N, 1}\right) \psi\left(1-\bar{\lambda}^{N, 2}\right)\right]} \\
\rho\left(\alpha^{B R} \mid \widehat{\delta}=\Delta^{2}\right)=0 \Leftrightarrow V^{S}\left(\rho=0, \alpha^{B R} \mid \widehat{\delta}=\Delta^{2}\right) \geq V^{S}\left(\rho=1, \alpha^{B R} \mid \widehat{\delta}=\Delta^{2}\right) \Leftrightarrow \\
\psi \Delta^{2}+\frac{X}{\phi} \geq \pi\left(\Delta^{1}+\Delta^{2}\right)+(1-\pi) \psi \Delta^{2}+X \Leftrightarrow \\
\Leftrightarrow \rho\left(\widehat{\delta}=\Delta^{2} \mid \alpha^{B R}\right)^{B R}=\left\{\begin{array}{lll}
0 & \text { if } & \phi \in\left[0, \frac{X}{\pi\left(\Delta^{1}+\Delta^{2}-\psi \Delta^{2}\right)+X}\right] \\
1 & \text { if } & \phi \in\left[\frac{X}{\pi\left(\Delta^{1}+\Delta^{2}-\psi \Delta^{2}\right)+X}, 1\right]
\end{array}\right.
\end{gathered}
$$

(d) in $(\widehat{\delta}=0)$ the selectorate's beliefs are

$$
\begin{gathered}
\mu(C \mid \widehat{\delta}=0)=\frac{\pi\left[\left(1-\bar{\lambda}^{C, 1}\right)(1-\psi)\left(1-\bar{\lambda}^{C, 2}\right)\right]}{\pi\left[\left(1-\bar{\lambda}^{C, 1}\right)(1-\psi)\left(1-\bar{\lambda}^{C, 2}\right)\right]+(1-\pi)\left[\left(1-\bar{\lambda}^{N, 1}\right)(1-\psi)\left(1-\bar{\lambda}^{N, 2}\right)\right]} \in[0,1]=0 \\
\rho\left(\alpha^{B R} \mid \widehat{\delta}=0\right)=0 \Leftrightarrow V^{S}\left(\rho=0, \alpha^{B R} \mid \widehat{\delta}=0\right) \geq V^{S}\left(\rho=1, \alpha^{B R} \mid \widehat{\delta}=0\right) \Leftrightarrow \\
\Leftrightarrow \frac{X}{\phi} \geq \pi\left(\Delta^{1}+\Delta^{2}\right)-\pi \psi \Delta^{2}+X \Leftrightarrow \phi \leq \frac{X}{\pi\left(\Delta^{1}+\Delta^{2}-\psi \Delta^{2}\right)+X} \Leftrightarrow \\
\Leftrightarrow \rho\left(\widehat{\delta}=0 \mid \alpha^{B R}\right)^{B R}=\left\{\begin{array}{lll}
0 & \text { if } & \phi \in\left[0, \frac{X}{\pi\left(\Delta^{1}+\Delta^{2}-\psi \Delta^{2}\right)+X}\right] \\
1 & \text { if } & \phi \in\left[\frac{X}{\pi\left(\Delta^{1}+\Delta^{2}-\psi \Delta^{2}\right)+X}, 1\right]
\end{array}\right.
\end{gathered}
$$


The Non Congruent Leader Now, we have to check whether the strategy profile $\left(\bar{\lambda}^{C, 1}, \bar{\lambda}^{C, 2}, \bar{\lambda}^{N, 1}, \bar{\lambda}^{N, 2}\right)=$ $(1,1,0,1)$ is part of of a SE. Her payoff will depend on $\rho^{B R}$ and $\alpha^{B R}$, hence on the basis of the previous $\alpha(\widehat{\delta}, \widehat{\rho})^{B R}$ and $\rho\left(\widehat{\delta} \mid \alpha^{B R}\right)^{B R}$ we need to distinguish five regions in the space $(\zeta, \phi) \in[0,1] \times[0,1]:$

1. if $(\zeta, \phi) \in\left[\frac{1}{X-\left(\Delta^{1}+\Delta^{2}\right)}, 1\right] \times[0,1]$ thus

$$
V^{N}\left(1,1,0,1 ; \rho^{B R}, \alpha^{B R}\right)=\Delta^{2}+r_{1}^{1}+\frac{X}{\phi}+0
$$

while deviating she get

$$
V^{N}\left(\bar{\lambda}^{C, 1}, \bar{\lambda}^{C, 2}, \bar{\lambda}^{N, 1}, \bar{\lambda}^{N, 2} ; \rho^{B R}, \alpha^{B R}\right)= \begin{cases}\psi \Delta^{2}+r_{1}^{1}+r_{1}^{2}+\frac{X}{\phi}+0 & \text { if } \bar{\lambda}^{C, 1}, \bar{\lambda}^{C, 2}, \bar{\lambda}^{N, 1}, \bar{\lambda}^{N, 2}=1,1,0,0 \\ \psi \Delta^{2}+\Delta^{1}+r_{1}^{2}+\frac{X}{\phi}+0 & \text { if } \bar{\lambda}^{C, 1}, \bar{\lambda}^{C, 2}, \bar{\lambda}^{N, 1}, \bar{\lambda}^{N, 2}=1,1,1,0 \\ \Delta^{2}+\Delta^{1}+\frac{X}{\phi}+0 & \text { if } \bar{\lambda}^{C, 1}, \bar{\lambda}^{C, 2}, \bar{\lambda}^{N, 1}, \bar{\lambda}^{N, 2}=1,1,1,1\end{cases}
$$

which is always greater for $\left(\bar{\lambda}^{N, 1}, \bar{\lambda}^{N, 2}\right)=(0,0)$, hence in this region this is not part of an equilibrium;

2. $(\zeta, \phi) \in\left[\frac{1}{X-\left[\pi\left(\Delta^{1}+\Delta^{2}\right)+(1-\pi) \psi \Delta^{2}\right]}, \frac{1}{X-\left(\Delta^{1}+\Delta^{2}\right)}\right] \times[0,1]$ thus

$$
V^{N}\left(1,1,0,1 ; \rho^{B R}, \alpha^{B R}\right)=\Delta^{2}+r_{1}^{1}+\frac{X}{\phi}+0
$$

while deviating she get

$$
\begin{gathered}
V^{N}\left(\bar{\lambda}^{C, 1}, \bar{\lambda}^{C, 2}, \bar{\lambda}^{N, 1}, \bar{\lambda}^{N, 2} ; \rho^{B R}, \alpha^{B R}\right)= \\
= \begin{cases}\psi \Delta^{2}+r_{1}^{1}+r_{1}^{2}+\frac{X}{\phi}+0 & \text { if } \bar{\lambda}^{C, 1}, \bar{\lambda}^{C, 2}, \bar{\lambda}^{N, 1}, \bar{\lambda}^{N, 2}=1,1,0,0 \\
\psi \Delta^{2}+\Delta^{1}+r_{1}^{2}+\frac{X}{\phi}+\psi\left[\psi \Delta^{2}+E\left(r_{2}^{1}\right)+E\left(r_{2}^{2}\right)+\frac{X}{\phi}\right] & \text { if } \bar{\lambda}^{C, 1}, \bar{\lambda}^{C, 2}, \bar{\lambda}^{N, 1}, \bar{\lambda}^{N, 2}=1,1,1,0 \\
\Delta^{2}+\Delta^{1}+\frac{X}{\phi}+\psi \Delta^{2}+E\left(r_{2}^{1}\right)+E\left(r_{2}^{2}\right)+\frac{X}{\phi} & \text { if } \bar{\lambda}^{C, 1}, \bar{\lambda}^{C, 2}, \bar{\lambda}^{N, 1}, \bar{\lambda}^{N, 2}=1,1,1,1 .\end{cases}
\end{gathered}
$$

Then, the non congruent leader has an incentives to deviate and it can't be part of an equilibrium

3. $(\zeta, \phi) \in\left[\frac{1}{X-\psi \Delta^{2}}, \frac{1}{X-\left[\pi\left(\Delta^{1}+\Delta^{2}\right)+(1-\pi) \psi \Delta^{2}\right]}\right] \times[0,1]$ thus

$$
V^{N}\left(1,1,0,1 ; \rho^{B R}, \alpha^{B R}\right)=\Delta^{2}+r_{1}^{1}+\frac{X}{\phi}+0
$$

while deviating she get

$$
\begin{gathered}
V^{N}\left(\bar{\lambda}^{C, 1}, \bar{\lambda}^{C, 2}, \bar{\lambda}^{N, 1}, \bar{\lambda}^{N, 2} ; \rho^{B R}, \alpha^{B R}\right)= \\
=\left\{\begin{array}{lr}
\psi \Delta^{2}+r_{1}^{1}+r_{1}^{2}+\frac{X}{\phi} & \text { if } \bar{\lambda}^{C, 1}, \bar{\lambda}^{C, 2}, \bar{\lambda}^{N, 1}, \bar{\lambda}^{N, 2}=1,1,0,0 \\
\psi \Delta^{2}+\Delta^{1}+r_{1}^{2}+\frac{X}{\phi} & \text { if } \bar{\lambda}^{C, 1}, \bar{\lambda}^{C, 2}, \bar{\lambda}^{N, 1}, \bar{\lambda}^{N, 2}=1,1,1,0 \\
\Delta^{2}+\Delta^{1}+\frac{X}{\phi}+\psi \Delta^{2}+E\left(r_{2}^{1}\right)+E\left(r_{2}^{2}\right)+\frac{X}{\phi} & \text { if } \bar{\lambda}^{C, 1}, \bar{\lambda}^{C, 2}, \bar{\lambda}^{N, 1}, \bar{\lambda}^{N, 2}=1,1,1,1 .
\end{array}\right.
\end{gathered}
$$


Then, the non congruent leader has an incentives to deviate, hence it can't be part of an equilibrium.

4. $(\zeta, \phi) \in\left[0, \frac{1}{X-\psi \Delta^{2}}\right] \times\left[0, \frac{X}{\pi\left(\Delta^{1}+\Delta^{2}-\psi \Delta^{2}\right)+X}\right]$ thus

$$
V^{N}\left(1,1,0,1 ; \rho^{B R}, \alpha^{B R}\right)=\Delta^{2}+r_{1}^{1}+\frac{X}{\phi}+\psi \Delta^{2}+E\left(r_{2}^{1}\right)+E\left(r_{2}^{2}\right)+\frac{X}{\phi}
$$

while deviating she get

$$
\begin{gathered}
V^{N}\left(\bar{\lambda}^{C, 1}, \bar{\lambda}^{C, 2}, \bar{\lambda}^{N, 1}, \bar{\lambda}^{N, 2} ; \rho^{B R}, \alpha^{B R}\right)= \\
= \begin{cases}\psi \Delta^{2}+r_{1}^{1}+r_{1}^{2}+\frac{X}{\phi}+\psi \Delta^{2}+E\left(r_{2}^{1}\right)+E\left(r_{2}^{2}\right)+\frac{X}{\phi} & \text { if } \bar{\lambda}^{C, 1}, \bar{\lambda}^{C, 2}, \bar{\lambda}^{N, 1}, \bar{\lambda}^{N, 2}=1,1,0,0 \\
\psi \Delta^{2}+\Delta^{1}+r_{1}^{2}+\frac{X}{\phi}+\psi \Delta^{2}+E\left(r_{2}^{1}\right)+E\left(r_{2}^{2}\right)+\frac{X}{\phi} & \text { if } \bar{\lambda}^{C, 1}, \bar{\lambda}^{C, 2}, \bar{\lambda}^{N, 1}, \bar{\lambda}^{N, 2}=1,1,1,0 \\
\Delta^{2}+\Delta^{1}+\frac{X}{\phi}+\psi \Delta^{2}+E\left(r_{2}^{1}\right)+E\left(r_{2}^{2}\right)+\frac{X}{\phi} & \text { if } \bar{\lambda}^{C, 1}, \bar{\lambda}^{C, 2}, \bar{\lambda}^{N, 1}, \bar{\lambda}^{N, 2}=1,1,1,1 .\end{cases}
\end{gathered}
$$

Then, the non congruent leader has an incentives to deviate to

$$
\left(\bar{\lambda}^{N, 1}, \bar{\lambda}^{N, 2}\right)=(0,0)
$$

hence, it can't be part of an equilibrium.

5. $(\zeta, \phi) \in\left[0, \frac{1}{X-\psi \Delta^{2}}\right] \times\left[\frac{X}{\pi\left(\Delta^{1}+\Delta^{2}\right)+X}, 1\right]$ thus

$$
V^{N}\left(1,1,0,1 ; \rho^{B R}, \alpha^{B R}\right)=\Delta^{2}+r_{1}^{1}+\frac{X}{\phi}+0
$$

while deviating she get

$$
\begin{gathered}
V^{N}\left(\bar{\lambda}^{C, 1}, \bar{\lambda}^{C, 2}, \bar{\lambda}^{N, 1}, \bar{\lambda}^{N, 2} ; \rho^{B R}, \alpha^{B R}\right)= \\
= \begin{cases}\psi \Delta^{2}+r_{1}^{1}+r_{1}^{2}+\frac{X}{\phi} & \text { if } \bar{\lambda}^{C, 1}, \bar{\lambda}^{C, 2}, \bar{\lambda}^{N, 1}, \bar{\lambda}^{N, 2}=1,1,0,0 \\
\psi \Delta^{2}+\Delta^{1}+r_{1}^{2}+\frac{X}{\phi}+\psi\left[\psi \Delta^{2}+E\left(r_{2}^{1}\right)+E\left(r_{2}^{2}\right)+\frac{X}{\phi}\right] & \text { if } \bar{\lambda}^{C, 1}, \bar{\lambda}^{C, 2}, \bar{\lambda}^{N, 1}, \bar{\lambda}^{N, 2}=1,1,1,0 \\
\Delta^{2}+\Delta^{1}+\frac{X}{\phi}+\psi \Delta^{2}+E\left(r_{2}^{1}\right)+E\left(r_{2}^{2}\right)+\frac{X}{\phi} & \text { if } \bar{\lambda}^{C, 1}, \bar{\lambda}^{C, 2}, \bar{\lambda}^{N, 1}, \bar{\lambda}^{N, 2}=1,1,1,1 .\end{cases}
\end{gathered}
$$

Then, the non congruent leader has an incentives to deviate, hence it can't be part of an equilibrium.

4. Strategy profile $\left(\bar{\lambda}^{C, 1}, \bar{\lambda}^{C, 2}, \bar{\lambda}^{N, 1}, \bar{\lambda}^{N, 2}\right)=(1,1,1,1)$

The Citizens After knowing their first-period utility and the selectorate's choice at the end of the first period, the citizens choose between revolt $(\alpha=1)$ or not $(\alpha=0)$. This means that to derive the citizens' sequential rational behavior, we should consider eight possible information sets, where in each information set, there are two decision nodes depending on the type of leader. 
Let $V^{Z}(\alpha \mid \delta, \rho)$ be the expected continuation payoff for the citizens when they choose $\alpha$ if $(\delta, \rho)$ has been observed. The expected continuation utility that the citizens will get after they choose to initiate a revolution in $(\delta, \rho)$ is:

$$
V^{Z}(\alpha=1 \mid \delta, \rho)=(1-\phi) \times \frac{X-(\zeta)^{-1}}{1-\phi}+\phi \times 0=X-(\zeta)^{-1} .
$$

Clearly, this payoff does not depend on their beliefs about the leader's type and thus on $(\delta, \rho)$. On the other hand, if the citizens decide to accommodate, the continuation payoff will depend on their beliefs about the type of leader which, in turn, will depend on their information at the time of deciding. Therefore, to find the citizens' rational behavior, we need to consider eight possible information sets.

1. Information set $\left(\widehat{\delta}=\Delta^{1}+\Delta^{2}, \widehat{\rho}=0\right)$ In this information set the players' beliefs are $\mu\left(C \mid \widehat{\delta}=\Delta^{1}+\Delta^{2}\right)=\frac{\pi\left[\bar{\lambda}^{C, 1} \bar{\lambda}^{C, 2}+\bar{\lambda}^{C, 1} \psi\left(1-\bar{\lambda}^{C, 2}\right)\right]}{\pi\left[\bar{\lambda}^{C, 1} \bar{\lambda}^{C, 2}+\lambda \psi\left(1-\bar{\lambda}^{C, 2}\right)\right]+(1-\pi)\left[\bar{\lambda}^{N, 1} \bar{\lambda}^{N, 2}+\bar{\lambda}^{N, 1} \psi\left(1-\bar{\lambda}^{N, 2}\right)\right]}=\pi$ The expected continuation payoff the citizens will get after they choose not to revolt is

$$
V^{Z}\left(\alpha=0 \mid \widehat{\delta}=\Delta^{1}+\Delta^{2}, \widehat{\rho}=0\right)=\pi\left(\Delta^{1}+\Delta^{2}\right)+(1-\pi) \psi \Delta^{2} .
$$

then sequential rationality implies that the citizens will choose to revolt in $\left(\widehat{\delta}=\Delta^{1}+\Delta^{2}, \widehat{\rho}=0\right)$ if and only if

$$
\begin{gathered}
V^{Z}\left(\alpha=1 \mid \widehat{\delta}=\Delta^{1}+\Delta^{2}, \widehat{\rho}=0\right) \geq V^{Z}\left(\alpha=0 \mid \widehat{\delta}=\Delta^{1}+\Delta^{2}, \widehat{\rho}=0\right) \Leftrightarrow \\
\Leftrightarrow \alpha\left(\widehat{\delta}=\Delta^{1}+\Delta^{2}, \widehat{\rho}=0\right)=1 \Leftrightarrow(\zeta)^{-1} \leq X-\left[\pi\left(\Delta^{1}+\Delta^{2}\right)+(1-\pi) \psi \Delta^{2}\right] .
\end{gathered}
$$

2. Information set $\left(\widehat{\delta}=\Delta^{1}+\Delta^{2}, \widehat{\rho}=1\right)$ In this information set, the incumbent leader is removed from office by the selectorate; therefore, there is a new leader and thus the expected utility the citizens will get after they choose not to revolt does not depend on the previous observation on $\widehat{\delta}$. Then, the expected utility the citizens will get after they choose not to revolt is:

$$
V^{Z}\left(\alpha=0 \mid \widehat{\delta}=\Delta^{1}+\Delta^{2}, \widehat{\rho}=1\right)=\pi\left(\Delta^{1}+\Delta^{2}\right)+(1-\pi) \psi \Delta^{2} .
$$

Sequential rationality implies that the citizens will choose to revolt in $\left(\widehat{\delta}=\Delta^{1}+\Delta^{2}, \widehat{\rho}=1\right)$ if and only if

$$
\begin{gathered}
V^{Z}\left(\alpha=1 \mid \widehat{\delta}=\Delta^{1}+\Delta^{2}, \widehat{\rho}=1\right) \geq V^{Z}\left(\alpha=0 \mid \widehat{\delta}=\Delta^{1}+\Delta^{2}, \widehat{\rho}=1\right) \Leftrightarrow \\
\Leftrightarrow \alpha\left(\widehat{\delta}=\Delta^{1}+\Delta^{2}, \widehat{\rho}=1\right)=1 \Leftrightarrow(\zeta)^{-1} \leq X-\left[\pi\left(\Delta^{1}+\Delta^{2}\right)+(1-\pi) \psi \Delta^{2}\right] .
\end{gathered}
$$


3. Information set $\left(\widehat{\delta}=\Delta^{1}, \widehat{\rho}=0\right)$ In this information set the players' beliefs are

$$
\mu\left(C \mid \widehat{\delta}=\Delta^{1}\right)=\frac{\pi\left[\bar{\lambda}^{C, 1}(1-\psi)\left(1-\bar{\lambda}^{C, 2}\right)\right]}{\pi\left[\bar{\lambda}^{C, 1}(1-\psi)\left(1-\bar{\lambda}^{C, 2}\right)\right]+(1-\pi)\left[\bar{\lambda}^{N, 1}(1-\psi)\left(1-\bar{\lambda}^{N, 2}\right)\right]} \in[0,1]=0
$$

and the expected continuation payoff the citizens will get after they choose not to revolt is

$$
V^{Z}\left(\alpha=0 \mid \widehat{\delta}=\Delta^{1}, \widehat{\rho}=0\right)=\mu\left(C \mid \widehat{\delta}=\Delta^{1}\right)\left(\Delta^{1}+\Delta^{2}\right)+\left[1-\mu\left(C \mid \widehat{\delta}=\Delta^{1}\right)\right] \psi \Delta^{2}=\psi \Delta^{2}
$$

Thus sequential rationality implies that the citizens will choose to revolt in $\left(\widehat{\delta}=\Delta^{2}, \widehat{\rho}=0\right)$ if and only if

$$
\begin{gathered}
V^{Z}\left(\alpha=1 \mid \widehat{\delta}=\Delta^{2}, \widehat{\rho}=0\right) \geq V^{Z}\left(\alpha=0 \mid \widehat{\delta}=\Delta^{2}, \widehat{\rho}=0\right) \Leftrightarrow \\
\Leftrightarrow \alpha\left(\widehat{\delta}=\Delta^{2}, \widehat{\rho}=0\right)=1 \Leftrightarrow X-(\zeta)^{-1} \geq \psi \Delta^{2} \Leftrightarrow(\zeta)^{-1} \leq X-\psi \Delta^{2} .
\end{gathered}
$$

4. Information set $\left(\widehat{\delta}=\Delta^{1}, \widehat{\rho}=1\right)$ In this information set, the incumbent leader is removed from office by the selectorate; therefore, there is a new leader and thus the expected utility the citizens will get after they choose not to revolt does not depend on the previous observation on $\widehat{\delta}$. Then, the expected utility the citizens will get after they choose not to revolt is:

$$
V^{Z}\left(\alpha=0 \mid \widehat{\delta}=\Delta^{1}, \widehat{\rho}=1\right)=\pi\left(\Delta^{1}+\Delta^{2}\right)+(1-\pi) \psi \Delta^{2} .
$$

Sequential rationality implies that the citizens will choose to revolt in $\left(\widehat{\delta}=\Delta^{1}, \widehat{\rho}=1\right)$ if and only if

$$
\begin{gathered}
V^{Z}\left(\alpha=1 \mid \widehat{\delta}=\Delta^{1}, \widehat{\rho}=1\right) \geq V^{Z}\left(\alpha=0 \mid \widehat{\delta}=\Delta^{1}, \widehat{\rho}=1\right) \Leftrightarrow \\
\Leftrightarrow \alpha\left(\widehat{\delta}=\Delta^{1}, \widehat{\rho}=1\right)=1 \Leftrightarrow(\zeta)^{-1} \leq X-\left[\pi\left(\Delta^{1}+\Delta^{2}\right)+(1-\pi) \psi \Delta^{2}\right] .
\end{gathered}
$$

5. Information set $\left(\widehat{\delta}=\Delta^{2}, \widehat{\rho}=0\right)$ In this information set the players' beliefs are

$$
\begin{gathered}
\mu\left(C \mid \widehat{\delta}=\Delta^{2}\right)= \\
=\frac{\pi\left[\left(1-\bar{\lambda}^{C, 1}\right) \bar{\lambda}^{C, 2}+\left(1-\bar{\lambda}^{C, 1}\right) \psi\left(1-\bar{\lambda}^{C, 2}\right)\right]}{\pi\left[\left(1-\bar{\lambda}^{C, 1}\right) \bar{\lambda}^{C, 2}+\left(1-\bar{\lambda}^{C, 1}\right) \psi\left(1-\bar{\lambda}^{C, 2}\right)\right]+(1-\pi)\left[\left(1-\bar{\lambda}^{N, 1}\right) \bar{\lambda}^{N, 2}+\left(1-\bar{\lambda}^{N, 1}\right) \psi\left(1-\bar{\lambda}^{N, 2}\right)\right]} \in[0,1]=0
\end{gathered}
$$

and the expected continuation payoff the citizens will get after they choose not to revolt is

$$
V^{Z}\left(\alpha=0 \mid \widehat{\delta}=\Delta^{2}, \widehat{\rho}=0\right)=\mu\left(C \mid \widehat{\delta}=\Delta^{2}\right)\left(\Delta^{1}+\Delta^{2}\right)+\left[1-\mu\left(C \mid \widehat{\delta}=\Delta^{2}\right)\right] \psi \Delta^{2}=\psi \Delta^{2}
$$


Thus sequential rationality implies that the citizens will choose to revolt in $\left(\widehat{\delta}=\Delta^{2}, \widehat{\rho}=0\right)$ if and only if

$$
\begin{gathered}
V^{Z}\left(\alpha=1 \mid \widehat{\delta}=\Delta^{2}, \widehat{\rho}=0\right) \geq V^{Z}\left(\alpha=0 \mid \widehat{\delta}=\Delta^{2}, \widehat{\rho}=0\right) \Leftrightarrow \\
\Leftrightarrow \alpha\left(\widehat{\delta}=\Delta^{2}, \widehat{\rho}=0\right)=1 \Leftrightarrow X-(\zeta)^{-1} \geq \psi \Delta^{2} \Leftrightarrow(\zeta)^{-1} \leq X-\psi \Delta^{2} .
\end{gathered}
$$

6. Information set $\left(\widehat{\delta}=\Delta^{2}, \widehat{\rho}=1\right)$ In this information set, the incumbent leader is removed from office by the selectorate; therefore, there is a new leader and thus the expected utility the citizens will get after they choose not to revolt does not depend on the previous observation on $\widehat{\delta}$. Then, the expected utility the citizens will get after they choose not to revolt is:

$$
V^{Z}\left(\alpha=0 \mid \widehat{\delta}=\Delta^{2}, \widehat{\rho}=1\right)=\pi\left(\Delta^{1}+\Delta^{2}\right)+(1-\pi) \psi \Delta^{2} .
$$

Sequential rationality implies that the citizens will choose to revolt in $\left(\widehat{\delta}=\Delta^{1}, \widehat{\rho}=1\right)$ if and only if

$$
\begin{gathered}
V^{Z}\left(\alpha=0 \mid \widehat{\delta}=\Delta^{2}, \widehat{\rho}=1\right) \geq V^{Z}\left(\alpha=0 \mid \widehat{\delta}=\Delta^{2}, \widehat{\rho}=1\right) \Leftrightarrow \\
\Leftrightarrow \alpha\left(\widehat{\delta}=\Delta^{2}, \widehat{\rho}=1\right)=1 \Leftrightarrow(\zeta)^{-1} \leq X-\left[\pi\left(\Delta^{1}+\Delta^{2}\right)+(1-\pi) \psi \Delta^{2}\right] .
\end{gathered}
$$

7. Information set $(\widehat{\delta}=0, \widehat{\rho}=0)$ In this information set the players' beliefs are $\mu(C \mid \widehat{\delta}=0)=\frac{\pi\left[\left(1-\bar{\lambda}^{C, 1}\right)(1-\psi)\left(1-\bar{\lambda}^{C, 2}\right)\right]}{\pi\left[\left(1-\bar{\lambda}^{C, 1}\right)(1-\psi)\left(1-\bar{\lambda}^{C, 2}\right)\right]+(1-\pi)\left[\left(1-\bar{\lambda}^{N, 1}\right)(1-\psi)\left(1-\bar{\lambda}^{N, 2}\right)\right]} \in[0,1]=0$ and the expected continuation payoff the citizens will get after they choose not to revolt is

$$
V^{Z}(\alpha=0 \mid \widehat{\delta}=0, \widehat{\rho}=0)=\mu(C \mid \widehat{\delta}=0)\left(\Delta^{1}+\Delta^{2}\right)+[1-\mu(C \mid \widehat{\delta}=0)] \psi \Delta^{2}=\psi \Delta^{2}
$$

Thus sequential rationality implies that the citizens will choose to revolt in $\left(\widehat{\delta}=\Delta^{1}, \widehat{\rho}=0\right)$ if and only if

$$
\begin{gathered}
V^{Z}(\alpha=1 \mid \widehat{\delta}=0, \widehat{\rho}=0) \geq V^{Z}(\alpha=0 \mid \widehat{\delta}=0, \widehat{\rho}=0) \Leftrightarrow \\
\Leftrightarrow \alpha(\widehat{\delta}=0, \widehat{\rho}=0)=1 \Leftrightarrow X-(\zeta)^{-1} \geq \psi \Delta^{2} \Leftrightarrow(\zeta)^{-1} \leq X-\psi \Delta^{2} .
\end{gathered}
$$

8. Information set $(\widehat{\delta}=0, \widehat{\rho}=1)$ In this information set, the incumbent leader is removed from office by the selectorate; therefore, there is a new leader and thus the expected utility the citizens will get after they choose not to revolt does not depend on the previous observation on $\widehat{\delta}$. Then, the expected utility the citizens will get after they choose not to revolt is:

$$
V^{Z}(\alpha=0 \mid \widehat{\delta}=0, \widehat{\rho}=1)=\pi\left(\Delta^{1}+\Delta^{2}\right)+(1-\pi) \psi \Delta^{2} .
$$


Sequential rationality implies that the citizens will choose to revolt in $\left(\widehat{\delta}=\Delta^{1}, \widehat{\rho}=1\right)$ if and only if

$$
\begin{gathered}
V^{Z}(\alpha=0 \mid \widehat{\delta}=0, \widehat{\rho}=1) \geq V^{Z}(\alpha=0 \mid \widehat{\delta}=0, \widehat{\rho}=1) \Leftrightarrow \\
\Leftrightarrow \alpha(\widehat{\delta}=0, \widehat{\rho}=1)=1 \Leftrightarrow(\zeta)^{-1} \leq X-\left[\pi\left(\Delta^{1}+\Delta^{2}\right)+(1-\pi) \psi \Delta^{2}\right] .
\end{gathered}
$$

The Selectorate After knowing their first-period utility at the end of the first period, the selectorate chooses between coup $(\rho=1)$ or not $(\rho=0)$. This means that to derive the selectorate's sequential rational behavior, we should consider four possible information sets, where in each information set, there are two decision nodes depending on the type of leader. Let $V^{S}\left(\rho=1, \alpha^{B R} \mid \delta\right)$ be the expected continuation payoff for the selectorate in $\delta$ if he subverts the incumbent leader and the citizens will subsequently choose according to $\alpha^{B R}$. Note that in this case, the payoff does not depend on $\delta$ being either 0 or $\Delta^{1}$, or $\Delta^{2}$, or $\Delta^{1}+\Delta^{2}$ since the leader has been changed. Therefore

$$
V^{S}\left(\rho=1, \alpha^{B R} \mid \delta\right)=\left(1-\alpha^{B R}\right)\left[\pi\left(\Delta^{1}+\Delta^{2}\right)+(1-\pi) \psi \Delta^{2}+X\right]
$$

as the type of the newly picked up leader is unknown, she will produce $\Delta^{1}+\Delta^{2}$ with probability $\pi$ and $\psi \Delta^{2}$ (in expected terms) otherwise. Moreover, both types of leader will distribute the entire social revenue to the selectorate, but the members of the selectorate who ousted the leader, with probability $\phi$, will be included in the successor's selectorate getting the patronage $\frac{X}{\phi}$ in the second period.

On the other hand

$$
V^{S}\left(\rho=0, \alpha^{B R} \mid \delta\right)=\left(1-\alpha^{B R}\right)\left[\mu(C \mid \delta, \rho=0)\left(\Delta^{1}+\Delta^{2}\right)+[1-\mu(C \mid \delta, \rho=0)] \psi \Delta^{2}+\frac{X}{\phi}\right] .
$$

Let consider the three regions in the space $\zeta \in[0,1]$ :

1. $\zeta \in\left[\frac{1}{X-\left[\pi\left(\Delta^{1}+\Delta^{2}\right)+(1-\pi) \psi \Delta^{2}\right]}, 1\right]$ then, considering the four selectorate's information sets:

(a) in $\left(\widehat{\delta}=\Delta^{1}+\Delta^{2}\right)$ the selectorate's beliefs are

$$
\begin{aligned}
& \mu\left(C \mid \widehat{\delta}=\Delta^{1}+\Delta^{2}\right)=\frac{\pi\left[\bar{\lambda}^{C, 1} \bar{\lambda}^{C, 2}+\bar{\lambda}^{C, 1} \psi\left(1-\bar{\lambda}^{C, 2}\right)\right]}{\pi\left[\bar{\lambda}^{C, 1} \bar{\lambda}^{C, 2}+\lambda \psi\left(1-\bar{\lambda}^{C, 2}\right)\right]+(1-\pi)\left[\bar{\lambda}^{N, 1} \bar{\lambda}^{N, 2}+\bar{\lambda}^{N, 1} \psi\left(1-\bar{\lambda}^{N, 2}\right)\right]}= \\
& =\frac{\pi}{\pi+(1-\pi) \psi} \equiv \pi^{+} \\
& \rho\left(\alpha^{B R} \mid \widehat{\delta}=\Delta^{1}+\Delta^{2}\right)=0 \Leftrightarrow V^{S}\left(\rho=0, \alpha^{B R} \mid \widehat{\delta}=\Delta^{1}+\Delta^{2}\right) \geq V^{S}\left(\rho=1, \alpha^{B R} \mid \widehat{\delta}=\Delta^{1}+\Delta^{2}\right) \Leftrightarrow \\
& \Leftrightarrow\left(1-\alpha^{B R}\right)\left[\pi^{+}\left(\Delta^{1}+\Delta^{2}\right)+\left(1-\pi^{+}\right) \psi \Delta^{2}+\frac{X}{\phi}\right] \geq\left(1-\alpha^{B R}\right)\left[\pi\left(\Delta^{1}+\Delta^{2}\right)+(1-\pi) \psi \Delta^{2}+X\right] \Leftrightarrow
\end{aligned}
$$




$$
\Leftrightarrow 0 \geq 0 \Leftrightarrow \rho\left(\widehat{\delta}=\Delta^{1}+\Delta^{2} \mid \alpha^{B R}\right)^{B R} \in[0,1]
$$

(b) in $\left(\widehat{\delta}=\Delta^{1}\right)$ the selectorate's beliefs are

$$
\begin{gathered}
\mu\left(C \mid \widehat{\delta}=\Delta^{1}\right)=\frac{\pi\left[\bar{\lambda}^{C, 1}(1-\psi)\left(1-\bar{\lambda}^{C, 2}\right)\right]}{\pi\left[\bar{\lambda}^{C, 1}(1-\psi)\left(1-\bar{\lambda}^{C, 2}\right)\right]+(1-\pi)\left[\bar{\lambda}^{N, 1}(1-\psi)\left(1-\bar{\lambda}^{N, 2}\right)\right]}=0 \\
\rho\left(\alpha^{B R} \mid \widehat{\delta}=\Delta^{1}\right)=0 \Leftrightarrow V^{S}\left(\rho=0, \alpha^{B R} \mid \widehat{\delta}=\Delta^{1}\right) \geq V^{S}\left(\rho=1, \alpha^{B R} \mid \widehat{\delta}=\Delta^{1}\right) \Leftrightarrow \\
\Leftrightarrow\left(1-\alpha^{B R}\right)\left[\psi \Delta^{2}+\frac{X}{\phi}\right] \geq\left(1-\alpha^{B R}\right)\left[\pi\left(\Delta^{1}+\Delta^{2}\right)+(1-\pi) \psi \Delta^{2}+X\right] \Leftrightarrow 0 \geq 0 \Leftrightarrow \\
\Leftrightarrow \rho\left(\widehat{\delta}=\Delta^{1} \mid \alpha^{B R}\right)^{B R} \in[0,1]
\end{gathered}
$$

(c) in $\left(\widehat{\delta}=\Delta^{2}\right)$ the selectorate's beliefs are

$$
\begin{gathered}
\mu\left(C \mid \widehat{\delta}=\Delta^{2}\right)= \\
=\frac{\pi\left[\left(1-\bar{\lambda}^{C, 1}\right) \bar{\lambda}^{C, 2}+\left(1-\bar{\lambda}^{C, 1}\right) \psi\left(1-\bar{\lambda}^{C, 2}\right)\right]}{\pi\left[\left(1-\bar{\lambda}^{C, 1}\right) \bar{\lambda}^{C, 2}+\left(1-\bar{\lambda}^{C, 1}\right) \psi\left(1-\bar{\lambda}^{C, 2}\right)\right]+(1-\pi)\left[\left(1-\bar{\lambda}^{N, 1}\right) \bar{\lambda}^{N, 2}+\left(1-\bar{\lambda}^{N, 1}\right) \psi\left(1-\bar{\lambda}^{N,}\right.\right.} \\
\in[0,1]=0 \\
\rho\left(\alpha^{B R} \mid \widehat{\delta}=\Delta^{2}\right)=0 \Leftrightarrow V^{S}\left(\rho=0, \alpha^{B R} \mid \widehat{\delta}=\Delta^{2}\right) \geq V^{S}\left(\rho=1, \alpha^{B R} \mid \widehat{\delta}=\Delta^{2}\right) \Leftrightarrow \\
\Leftrightarrow\left(1-\alpha^{B R}\right)\left[\psi \Delta^{2}+\frac{X}{\phi}\right] \geq\left(1-\alpha^{B R}\right)\left[\pi\left(\Delta^{1}+\Delta^{2}\right)+(1-\pi) \psi \Delta^{2}+X\right] \Leftrightarrow 0 \geq 0 \Leftrightarrow \\
\Leftrightarrow \rho\left(\widehat{\delta}=\Delta^{2} \mid \alpha^{B R}\right)^{B R} \in[0,1]
\end{gathered}
$$

(d) in $(\widehat{\delta}=0)$ the selectorate's beliefs are

$$
\begin{gathered}
\mu(C \mid \widehat{\delta}=0)=\frac{\pi\left[\left(1-\bar{\lambda}^{C, 1}\right)(1-\psi)\left(1-\bar{\lambda}^{C, 2}\right)\right]}{\pi\left[\left(1-\bar{\lambda}^{C, 1}\right)(1-\psi)\left(1-\bar{\lambda}^{C, 2}\right)\right]+(1-\pi)\left[\left(1-\bar{\lambda}^{N, 1}\right)(1-\psi)\left(1-\bar{\lambda}^{N, 2}\right)\right]} \in[0,1]=0 \\
\rho\left(\alpha^{B R} \mid \widehat{\delta}=0\right)=0 \Leftrightarrow V^{S}\left(\rho=0, \alpha^{B R} \mid \widehat{\delta}=0\right) \geq V^{S}\left(\rho=1, \alpha^{B R} \mid \widehat{\delta}=0\right) \Leftrightarrow \\
\Leftrightarrow\left(1-\alpha^{B R}\right)\left[\psi \Delta^{2}+\frac{X}{\phi}\right] \geq\left(1-\alpha^{B R}\right)\left[\pi\left(\Delta^{1}+\Delta^{2}\right)+(1-\pi) \psi \Delta^{2}+X\right] \Leftrightarrow 0 \geq 0 \Leftrightarrow \\
\Leftrightarrow \rho\left(\widehat{\delta}=0 \mid \alpha^{B R}\right)^{B R} \in[0,1]
\end{gathered}
$$

2. $\zeta \in\left[\frac{1}{X-\psi \Delta^{2}}, \frac{1}{X-\left[\pi\left(\Delta^{1}+\Delta^{2}\right)+(1-\pi) \psi \Delta^{2}\right]}\right]$ then, considering the four selectorate's information sets: 
(a) in $\left(\widehat{\delta}=\Delta^{1}+\Delta^{2}\right)$ the selectorate's beliefs are

$$
\begin{gathered}
\mu\left(C \mid \widehat{\delta}=\Delta^{1}+\Delta^{2}\right)=\frac{\pi\left[\bar{\lambda}^{C, 1} \bar{\lambda}^{C, 2}+\bar{\lambda}^{C, 1} \psi\left(1-\bar{\lambda}^{C, 2}\right)\right]}{\pi\left[\bar{\lambda}^{C, 1} \bar{\lambda}^{C, 2}+\lambda \psi\left(1-\bar{\lambda}^{C, 2}\right)\right]+(1-\pi)\left[\bar{\lambda}^{N, 1} \bar{\lambda}^{N, 2}+\bar{\lambda}^{N, 1} \psi\left(1-\bar{\lambda}^{N, 2}\right)\right]}=\pi \\
\rho\left(\alpha^{B R} \mid \widehat{\delta}=\Delta^{1}+\Delta^{2}\right)=0 \Leftrightarrow V^{S}\left(\rho=0, \alpha^{B R} \mid \widehat{\delta}=\Delta^{1}+\Delta^{2}\right) \geq V^{S}\left(\rho=1, \alpha^{B R} \mid \widehat{\delta}=\Delta^{1}+\Delta^{2}\right) \Leftrightarrow \\
\Leftrightarrow \pi\left(\Delta^{1}+\Delta^{2}\right)+(1-\pi) \psi \Delta^{2}+\frac{X}{\phi} \geq \pi\left(\Delta^{1}+\Delta^{2}\right)+(1-\pi) \psi \Delta^{2}+X \Leftrightarrow \\
\Leftrightarrow \rho\left(\widehat{\delta}=\Delta^{1}+\Delta^{2} \mid \alpha^{B R}\right)^{B R}=0
\end{gathered}
$$

(b) in $\left(\widehat{\delta}=\Delta^{1}\right)$ the selectorate's beliefs are

$$
\begin{gathered}
\mu\left(C \mid \widehat{\delta}=\Delta^{1}\right)=\frac{\pi\left[\bar{\lambda}^{C, 1}(1-\psi)\left(1-\bar{\lambda}^{C, 2}\right)\right]}{\pi\left[\bar{\lambda}^{C, 1}(1-\psi)\left(1-\bar{\lambda}^{C, 2}\right)\right]+(1-\pi)\left[\bar{\lambda}^{N, 1}(1-\psi)\left(1-\bar{\lambda}^{N, 2}\right)\right]} \in[0,1]=0 \\
\rho\left(\alpha^{B R} \mid \widehat{\delta}=\Delta^{1}\right)=0 \Leftrightarrow V^{S}\left(\rho=0, \alpha^{B R} \mid \widehat{\delta}=\Delta^{1}\right) \geq V^{S}\left(\rho=1, \alpha^{B R} \mid \widehat{\delta}=\Delta^{1}\right) \Leftrightarrow \\
\Leftrightarrow 0 \geq \pi\left(\Delta^{1}+\Delta^{2}\right)+(1-\pi) \psi \Delta^{2}+X \Leftrightarrow \\
\Leftrightarrow \rho\left(\widehat{\delta}=\Delta^{1} \mid \alpha^{B R}\right)^{B R}=1
\end{gathered}
$$

(c) in $\left(\widehat{\delta}=\Delta^{2}\right)$ the selectorate's beliefs are

$$
\begin{gathered}
\mu\left(C \mid \widehat{\delta}=\Delta^{2}\right)= \\
=\frac{\pi\left[\left(1-\bar{\lambda}^{C, 1}\right) \bar{\lambda}^{C, 2}+\left(1-\bar{\lambda}^{C, 1}\right) \psi\left(1-\bar{\lambda}^{C, 2}\right)\right]}{\pi\left[\left(1-\bar{\lambda}^{C, 1}\right) \bar{\lambda}^{C, 2}+\left(1-\bar{\lambda}^{C, 1}\right) \psi\left(1-\bar{\lambda}^{C, 2}\right)\right]+(1-\pi)\left[\left(1-\bar{\lambda}^{N, 1}\right) \bar{\lambda}^{N, 2}+\left(1-\bar{\lambda}^{N, 1}\right) \psi\left(1-\bar{\lambda}^{N,},\right.\right.} \\
\in[0,1]=0 \\
\rho\left(\alpha^{B R} \mid \widehat{\delta}=\Delta^{2}\right)=0 \Leftrightarrow V^{S}\left(\rho=0, \alpha^{B R} \mid \widehat{\delta}=\Delta^{2}\right) \geq V^{S}\left(\rho=1, \alpha^{B R} \mid \widehat{\delta}=\Delta^{2}\right) \Leftrightarrow \\
\Leftrightarrow 0 \geq \pi\left(\Delta^{1}+\Delta^{2}\right)+(1-\pi) \psi \Delta^{2}+X \Leftrightarrow \\
\Leftrightarrow \rho\left(\widehat{\delta}=\Delta^{2} \mid \alpha^{B R}\right)^{B R}=1
\end{gathered}
$$

(d) in $(\widehat{\delta}=0)$ the selectorate's beliefs are

$$
\begin{gathered}
\mu(C \mid \widehat{\delta}=0)=\frac{\pi\left[\left(1-\bar{\lambda}^{C, 1}\right)(1-\psi)\left(1-\bar{\lambda}^{C, 2}\right)\right]}{\pi\left[\left(1-\bar{\lambda}^{C, 1}\right)(1-\psi)\left(1-\bar{\lambda}^{C, 2}\right)\right]+(1-\pi)\left[\left(1-\bar{\lambda}^{N, 1}\right)(1-\psi)\left(1-\bar{\lambda}^{N, 2}\right)\right]} \in[0,1]=0 \\
\rho\left(\alpha^{B R} \mid \widehat{\delta}=0\right)=0 \Leftrightarrow V^{S}\left(\rho=0, \alpha^{B R} \mid \widehat{\delta}=0\right) \geq V^{S}\left(\rho=1, \alpha^{B R} \mid \widehat{\delta}=0\right) \Leftrightarrow
\end{gathered}
$$




$$
\begin{gathered}
\Leftrightarrow 0 \geq \pi\left(\Delta^{1}+\Delta^{2}\right)+(1-\pi) \psi \Delta^{2}+X \Leftrightarrow \\
\Leftrightarrow \rho\left(\widehat{\delta}=0 \mid \alpha^{B R}\right)^{B R}=1
\end{gathered}
$$

3. $\zeta \in\left[0, \frac{1}{X-\psi \Delta^{2}}\right]$ then, considering the four selectorate's information sets:

(a) in $\left(\widehat{\delta}=\Delta^{1}+\Delta^{2}\right)$ the selectorate's beliefs are

$$
\begin{gathered}
\mu\left(C \mid \widehat{\delta}=\Delta^{1}+\Delta^{2}\right)=\frac{\pi\left[\bar{\lambda}^{C, 1} \bar{\lambda}^{C, 2}+\bar{\lambda}^{C, 1} \psi\left(1-\bar{\lambda}^{C, 2}\right)\right]}{\pi\left[\bar{\lambda}^{C, 1} \bar{\lambda}^{C, 2}+\lambda \psi\left(1-\bar{\lambda}^{C, 2}\right)\right]+(1-\pi)\left[\bar{\lambda}^{N, 1} \bar{\lambda}^{N, 2}+\bar{\lambda}^{N, 1} \psi\left(1-\bar{\lambda}^{N, 2}\right)\right]}=\pi \\
\rho\left(\alpha^{B R} \mid \widehat{\delta}=\Delta^{1}+\Delta^{2}\right)=0 \Leftrightarrow V^{S}\left(\rho=0, \alpha^{B R} \mid \widehat{\delta}=\Delta^{1}+\Delta^{2}\right) \geq V^{S}\left(\rho=1, \alpha^{B R} \mid \widehat{\delta}=\Delta^{1}+\Delta^{2}\right) \Leftrightarrow \\
\Leftrightarrow \pi\left(\Delta^{1}+\Delta^{2}\right)+(1-\pi) \psi \Delta^{2}+\frac{X}{\phi} \geq \pi\left(\Delta^{1}+\Delta^{2}\right)+(1-\pi) \psi \Delta^{2}+X \Leftrightarrow \\
\Leftrightarrow \rho\left(\widehat{\delta}=\Delta^{1}+\Delta^{2} \mid \alpha^{B R}\right)^{B R}=0
\end{gathered}
$$

(b) in $\left(\widehat{\delta}=\Delta^{1}\right)$ the selectorate's beliefs are

$$
\begin{gathered}
\mu\left(C \mid \widehat{\delta}=\Delta^{1}\right)=\frac{\pi\left[\bar{\lambda}^{C, 1}(1-\psi)\left(1-\bar{\lambda}^{C, 2}\right)\right]}{\pi\left[\bar{\lambda}^{C, 1}(1-\psi)\left(1-\bar{\lambda}^{C, 2}\right)\right]+(1-\pi)\left[\bar{\lambda}^{N, 1}(1-\psi)\left(1-\bar{\lambda}^{N, 2}\right)\right]} \in[0,1]=0 \\
\rho\left(\alpha^{B R} \mid \widehat{\delta}=\Delta^{2}\right)=0 \Leftrightarrow V^{S}\left(\rho=0, \alpha^{B R} \mid \widehat{\delta}=\Delta^{2}\right) \geq V^{S}\left(\rho=1, \alpha^{B R} \mid \widehat{\delta}=\Delta^{2}\right) \Leftrightarrow \\
\Leftrightarrow \frac{X}{\phi} \geq \pi\left(\Delta^{1}+\Delta^{2}\right)-\pi \psi \Delta^{2}+X \Leftrightarrow \phi \leq \frac{X}{\pi\left(\Delta^{1}+\Delta^{2}-\psi \Delta^{2}\right)+X} \Leftrightarrow \\
\Leftrightarrow \rho\left(\widehat{\delta}=\Delta^{1} \mid \alpha^{B R}\right)^{B R}=\left\{\begin{array}{lll}
0 & \text { if } & \phi \in\left[0, \frac{X}{\pi\left(\Delta^{1}+\Delta^{2}-\psi \Delta^{2}\right)+X}\right] \\
1 & \text { if } & \phi \in\left[\frac{X}{\pi\left(\Delta^{1}+\Delta^{2}-\psi \Delta^{2}\right)+X}, 1\right]
\end{array}\right.
\end{gathered}
$$

(c) in $\left(\widehat{\delta}=\Delta^{2}\right)$ the selectorate's beliefs are

$$
\begin{gathered}
\mu\left(C \mid \widehat{\delta}=\Delta^{2}\right)= \\
=\frac{\pi\left[\left(1-\bar{\lambda}^{C, 1}\right) \bar{\lambda}^{C, 2}+\left(1-\bar{\lambda}^{C, 1}\right) \psi\left(1-\bar{\lambda}^{C, 2}\right)\right]}{\pi\left[\left(1-\bar{\lambda}^{C, 1}\right) \bar{\lambda}^{C, 2}+\left(1-\bar{\lambda}^{C, 1}\right) \psi\left(1-\bar{\lambda}^{C, 2}\right)\right]+(1-\pi)\left[\left(1-\bar{\lambda}^{N, 1}\right) \bar{\lambda}^{N, 2}+\left(1-\bar{\lambda}^{N, 1}\right) \psi\left(1-\bar{\lambda}^{N,},\right.\right.} \\
\in[0,1]=0 \\
\rho\left(\alpha^{B R} \mid \widehat{\delta}=\Delta^{2}\right)=0 \Leftrightarrow V^{S}\left(\rho=0, \alpha^{B R} \mid \widehat{\delta}=\Delta^{2}\right) \geq V^{S}\left(\rho=1, \alpha^{B R} \mid \widehat{\delta}=\Delta^{2}\right) \Leftrightarrow \\
\Leftrightarrow \frac{X}{\phi} \geq \pi\left(\Delta^{1}+\Delta^{2}\right)-\pi \psi \Delta^{2}+X \Leftrightarrow \phi \leq \frac{X}{\pi\left(\Delta^{1}+\Delta^{2}-\psi \Delta^{2}\right)+X} \Leftrightarrow \\
\rho\left(\widehat{\delta}=\Delta^{2} \mid \alpha^{B R}\right)^{B R}=\left\{\begin{array}{lll}
0 & \text { if } \phi \in\left[0, \frac{X}{\pi\left(\Delta^{1}+\Delta^{2}-\psi \Delta^{2}\right)+X}\right] \\
1 & \text { if } \phi \in\left[\frac{X}{\pi\left(\Delta^{1}+\Delta^{2}-\psi \Delta^{2}\right)+X}, 1\right]
\end{array}\right.
\end{gathered}
$$


(d) in $(\widehat{\delta}=0)$ the selectorate's beliefs are

$$
\begin{gathered}
\mu(C \mid \widehat{\delta}=0)=\frac{\pi\left[\left(1-\bar{\lambda}^{C, 1}\right)(1-\psi)\left(1-\bar{\lambda}^{C, 2}\right)\right]}{\pi\left[\left(1-\bar{\lambda}^{C, 1}\right)(1-\psi)\left(1-\bar{\lambda}^{C, 2}\right)\right]+(1-\pi)\left[\left(1-\bar{\lambda}^{N, 1}\right)(1-\psi)\left(1-\bar{\lambda}^{N, 2}\right)\right]} \in[0,1]=0 \\
\rho\left(\alpha^{B R} \mid \widehat{\delta}=0\right)=0 \Leftrightarrow V^{S}\left(\rho=0, \alpha^{B R} \mid \widehat{\delta}=0\right) \geq V^{S}\left(\rho=1, \alpha^{B R} \mid \widehat{\delta}=0\right) \Leftrightarrow \\
\Leftrightarrow \frac{X}{\phi} \geq \pi\left(\Delta^{1}+\Delta^{2}\right)-\pi \psi \Delta^{2}+X \Leftrightarrow \phi \leq \frac{X}{\pi\left(\Delta^{1}+\Delta^{2}-\psi \Delta^{2}\right)+X} \Leftrightarrow \\
\rho\left(\widehat{\delta}=0 \mid \alpha^{B R}\right)^{B R}=\left\{\begin{array}{lll}
0 & \text { if } & \phi \in\left[0, \frac{X}{\pi\left(\Delta^{1}+\Delta^{2}-\psi \Delta^{2}\right)+X}\right] \\
1 & \text { if } & \phi \in\left[\frac{X}{\pi\left(\Delta^{1}+\Delta^{2}-\psi \Delta^{2}\right)+X}, 1\right]
\end{array}\right.
\end{gathered}
$$

The Non Congruent Leader Now, we have to check whether the strategy profile $\left(\bar{\lambda}^{C, 1}, \bar{\lambda}^{C, 2}, \bar{\lambda}^{N, 1}, \bar{\lambda}^{N, 2}\right)=$ $(1,1,1,1)$ is part of of a SE. Her payoff will depend on $\rho^{B R}$ and $\alpha^{B R}$, hence on the basis of the previous $\alpha(\widehat{\delta}, \widehat{\rho})^{B R}$ and $\rho\left(\widehat{\delta} \mid \alpha^{B R}\right)^{B R}$ we need to distinguish five regions in the space $(\zeta, \phi) \in[0,1] \times[0,1]:$

1. if $(\zeta, \phi) \in\left[\frac{1}{X-\left[\pi\left(\Delta^{1}+\Delta^{2}\right)+(1-\pi) \psi \Delta^{2}\right]}, 1\right] \times[0,1]$ thus

$$
V^{N}\left(1,1,1,1 ; \rho^{B R}, \alpha^{B R}\right)=\Delta^{2}+\Delta^{1}+\frac{X}{\phi}+0
$$

while deviating she get

$$
V^{N}\left(\bar{\lambda}^{C, 1}, \bar{\lambda}^{C, 2}, \bar{\lambda}^{N, 1}, \bar{\lambda}^{N, 2} ; \rho^{B R}, \alpha^{B R}\right)= \begin{cases}\psi \Delta^{2}+r_{1}^{1}+r_{1}^{2}+\frac{X}{\phi}+0 & \text { if } \bar{\lambda}^{C, 1}, \bar{\lambda}^{C, 2}, \bar{\lambda}^{N, 1}, \bar{\lambda}^{N, 2}=1,1,0,0 \\ \Delta^{2}+r_{1}^{1}+\frac{X}{\phi}+0 & \text { if } \bar{\lambda}^{C, 1}, \bar{\lambda}^{C, 2}, \bar{\lambda}^{N, 1}, \bar{\lambda}^{N, 2}=1,1,0,1 \\ \Delta^{1}+\psi \Delta^{2}+r_{1}^{2}+\frac{X}{\phi}+0 & \text { if } \bar{\lambda}^{C, 1}, \bar{\lambda}^{C, 2}, \bar{\lambda}^{N, 1}, \bar{\lambda}^{N, 2}=1,1,1,0\end{cases}
$$

which is always greater for $\left(\bar{\lambda}^{N, 1}, \bar{\lambda}^{N, 2}\right)=(0,0)$, hence in this region this is not part of an equilibrium;

2. $(\zeta, \phi) \in\left[\frac{1}{X-\psi \Delta^{2}}, \frac{1}{X-\left[\pi\left(\Delta^{1}+\Delta^{2}\right)+(1-\pi) \psi \Delta^{2}\right]}\right] \times[0,1]$ thus

$$
V^{N}\left(1,1,1,1 ; \rho^{B R}, \alpha^{B R}\right)=\Delta^{2}+\Delta^{1}+\frac{X}{\phi}+\psi \Delta^{2}+E\left(r_{2}^{1}\right)+E\left(r_{2}^{2}\right)+\frac{X}{\phi}
$$

while deviating she get

$$
\begin{gathered}
V^{N}\left(\bar{\lambda}^{C, 1}, \bar{\lambda}^{C, 2}, \bar{\lambda}^{N, 1}, \bar{\lambda}^{N, 2} ; \rho^{B R}, \alpha^{B R}\right)= \\
= \begin{cases}\psi \Delta^{2}+r_{1}^{1}+r_{1}^{2}+\frac{X}{\phi} & \text { if } \bar{\lambda}^{C, 1}, \bar{\lambda}^{C, 2}, \bar{\lambda}^{N, 1}, \bar{\lambda}^{N, 2}=1,1,0,0 \\
\Delta^{2}+r_{1}^{1}+\frac{X}{\phi} & \text { if } \bar{\lambda}^{C, 1}, \bar{\lambda}^{C, 2}, \bar{\lambda}^{N, 1}, \bar{\lambda}^{N, 2}=1,1,0,1 \\
\psi \Delta^{2}+\Delta^{1}+r_{1}^{2}+\frac{X}{\phi}+\psi\left[\psi \Delta^{2}+E\left(r_{2}^{1}\right)+E\left(r_{2}^{2}\right)+\frac{X}{\phi}\right] & \text { if } \bar{\lambda}^{C, 1}, \bar{\lambda}^{C, 2}, \bar{\lambda}^{N, 1}, \bar{\lambda}^{N, 2}=1,1,1,0 .\end{cases}
\end{gathered}
$$


Then, the non congruent leader has no incentives to deviate if and only if

$$
\begin{gathered}
\left\{\begin{array}{l}
\psi \Delta^{2}+r_{1}^{1}+r_{1}^{2}+\frac{X}{\phi} \leq \Delta^{2}+\Delta^{1}+\frac{X}{\phi}+\psi \Delta^{2}+E\left(r_{2}^{1}\right)+E\left(r_{2}^{2}\right)+\frac{X}{\phi} \\
\Delta^{2}+\Delta^{1}+\frac{X}{\phi}+\psi \Delta^{2}+E\left(r_{2}^{1}\right)+E\left(r_{2}^{2}\right)+\frac{X}{\phi} \geq \psi \Delta^{2}+\Delta^{1}+r_{1}^{2}+\frac{X}{\phi}+\psi\left[\psi \Delta^{2}+E\left(r_{2}^{1}\right)+E\left(r_{2}^{2}\right)+\frac{X}{\phi}\right]
\end{array}\right. \\
\Leftrightarrow\left\{\begin{array}{l}
r_{1}^{1}+r_{1}^{2} \leq \Delta^{2}+\Delta^{1}+E\left(r_{2}^{1}\right)+E\left(r_{2}^{2}\right)+\frac{X}{\phi} \\
r_{1}^{2} \leq(1-\psi) \Delta^{2}+(1-\psi)\left[\psi \Delta^{2}+E\left(r_{2}^{1}\right)+E\left(r_{2}^{2}\right)+\frac{X}{\phi}\right]
\end{array}\right.
\end{gathered}
$$

Hence in this region the leader might choose

$$
\left(\bar{\lambda}^{C, 1}, \bar{\lambda}^{C, 2}, \bar{\lambda}^{N, 1}, \bar{\lambda}^{N, 2}\right)=(1,1,1,1)
$$

with probability

$$
\begin{gathered}
\mathbf{P}\left\{r_{1}^{1}+r_{1}^{2} \leq \Delta^{2}+\Delta^{1}+E\left(r_{2}^{1}\right)+E\left(r_{2}^{2}\right)+\frac{X}{\phi} \& r_{1}^{2} \leq(1-\psi) \Delta^{2}+(1-\psi)\left[\psi \Delta^{2}+E\left(r_{2}^{1}\right)+E\left(r_{2}^{2}\right)+\frac{X}{\phi}\right]\right\} \equiv \\
\equiv F E(\psi)
\end{gathered}
$$

however before to conclude we need to consider players' incentives with the other leader's strategies.

3. $(\zeta, \phi) \in\left[0, \frac{1}{X-\psi \Delta^{2}}\right] \times\left[0, \frac{X}{\pi\left(\Delta^{1}+\Delta^{2}-\psi \Delta^{2}\right)+X}\right]$ thus

$$
V^{N}\left(1,1,1,1 ; \rho^{B R}, \alpha^{B R}\right)=\Delta^{2}+\Delta^{1}+\frac{X}{\phi}+\psi \Delta^{2}+E\left(r_{2}^{1}\right)+E\left(r_{2}^{2}\right)+\frac{X}{\phi}
$$

while deviating she get

$$
\begin{gathered}
V^{N}\left(\bar{\lambda}^{C, 1}, \bar{\lambda}^{C, 2}, \bar{\lambda}^{N, 1}, \bar{\lambda}^{N, 2} ; \rho^{B R}, \alpha^{B R}\right)= \\
= \begin{cases}\psi \Delta^{2}+r_{1}^{1}+r_{1}^{2}+\frac{X}{\phi}+\psi \Delta^{2}+E\left(r_{2}^{1}\right)+E\left(r_{2}^{2}\right)+\frac{X}{\phi} & \text { if } \bar{\lambda}^{C, 1}, \bar{\lambda}^{C, 2}, \bar{\lambda}^{N, 1}, \bar{\lambda}^{N, 2}=1,1,0,0 \\
\Delta^{2}+r_{1}^{1}+\frac{X}{\phi}+\psi \Delta^{2}+E\left(r_{2}^{1}\right)+E\left(r_{2}^{2}\right)+\frac{X}{\phi} & \text { if } \bar{\lambda}^{C, 1}, \bar{\lambda}^{C, 2}, \bar{\lambda}^{N, 1}, \bar{\lambda}^{N, 2}=1,1,0,1 \\
\psi \Delta^{2}+\Delta^{1}+r_{1}^{2}+\frac{X}{\phi}+\psi \Delta^{2}+E\left(r_{2}^{1}\right)+E\left(r_{2}^{2}\right)+\frac{X}{\phi} & \text { if } \bar{\lambda}^{C, 1}, \bar{\lambda}^{C, 2}, \bar{\lambda}^{N, 1}, \bar{\lambda}^{N, 2}=1,1,1,0 .\end{cases}
\end{gathered}
$$

Then, the non congruent leader has an incentives to deviate to $\left(\bar{\lambda}^{N, 1}, \bar{\lambda}^{N, 2}\right)=(0,0)$, hence, it can't be part of an equilibrium;

4. $(\zeta, \phi) \in\left[0, \frac{1}{X-\psi \Delta^{2}}\right] \times\left[\frac{X}{\pi\left(\Delta^{1}+\Delta^{2}\right)+X}, 1\right]$ thus

$$
V^{N}\left(1,1,1,1 ; \rho^{B R}, \alpha^{B R}\right)=\Delta^{2}+\Delta^{1}+\frac{X}{\phi}+\psi \Delta^{2}+E\left(r_{2}^{1}\right)+E\left(r_{2}^{2}\right)+\frac{X}{\phi}
$$

while deviating she get

$$
V^{N}\left(\bar{\lambda}^{C, 1}, \bar{\lambda}^{C, 2}, \bar{\lambda}^{N, 1}, \bar{\lambda}^{N, 2} ; \rho^{B R}, \alpha^{B R}\right)=
$$




$$
= \begin{cases}\psi \Delta^{2}+r_{1}^{1}+r_{1}^{2}+\frac{X}{\phi} & \text { if } \bar{\lambda}^{C, 1}, \bar{\lambda}^{C, 2}, \bar{\lambda}^{N, 1}, \bar{\lambda}^{N, 2}=1,1,0,0 \\ \Delta^{2}+r_{1}^{1}+\frac{X}{\phi} & \text { if } \bar{\lambda}^{C, 1}, \bar{\lambda}^{C, 2}, \bar{\lambda}^{N, 1}, \bar{\lambda}^{N, 2}=1,1,0,1 \\ \psi \Delta^{2}+\Delta^{1}+r_{1}^{2}+\frac{X}{\phi}+\psi\left[\psi \Delta^{2}+E\left(r_{2}^{1}\right)+E\left(r_{2}^{2}\right)+\frac{X}{\phi}\right] & \text { if } \bar{\lambda}^{C, 1}, \bar{\lambda}^{C, 2}, \bar{\lambda}^{N, 1}, \bar{\lambda}^{N, 2}=1,1,1,0 .\end{cases}
$$

Then, the non congruent leader has no incentives to deviate if and only if

$$
\begin{gathered}
\left\{\begin{array}{l}
\psi \Delta^{2}+r_{1}^{1}+r_{1}^{2}+\frac{X}{\phi} \leq \Delta^{2}+\Delta^{1}+\frac{X}{\phi}+\psi \Delta^{2}+E\left(r_{2}^{1}\right)+E\left(r_{2}^{2}\right)+\frac{X}{\phi} \\
\Delta^{2}+\Delta^{1}+\frac{X}{\phi}+\psi \Delta^{2}+E\left(r_{2}^{1}\right)+E\left(r_{2}^{2}\right)+\frac{X}{\phi} \geq \psi \Delta^{2}+\Delta^{1}+r_{1}^{2}+\frac{X}{\phi}+\psi\left[\psi \Delta^{2}+E\left(r_{2}^{1}\right)+E\left(r_{2}^{2}\right)+\frac{X}{\phi}\right]
\end{array}\right. \\
\Leftrightarrow\left\{\begin{array}{l}
r_{1}^{1}+r_{1}^{2} \leq \Delta^{2}+\Delta^{1}+E\left(r_{2}^{1}\right)+E\left(r_{2}^{2}\right)+\frac{X}{\phi} \\
r_{1}^{2} \leq(1-\psi) \Delta^{2}+(1-\psi)\left[\psi \Delta^{2}+E\left(r_{2}^{1}\right)+E\left(r_{2}^{2}\right)+\frac{X}{\phi}\right]
\end{array}\right.
\end{gathered}
$$

Hence in this region we may get an equilibrium:

$$
\left(\bar{\lambda}^{C, 1}, \bar{\lambda}^{C, 2}, \bar{\lambda}^{N, 1}, \bar{\lambda}^{N, 2}\right)=(1,1,1,1)
$$

with probability

$$
\begin{gathered}
\mathbf{P}\left\{r_{1}^{1}+r_{1}^{2} \leq \Delta^{2}+\Delta^{1}+E\left(r_{2}^{1}\right)+E\left(r_{2}^{2}\right)+\frac{X}{\phi} \& r_{1}^{2} \leq(1-\psi) \Delta^{2}+(1-\psi)\left[\psi \Delta^{2}+E\left(r_{2}^{1}\right)+E\left(r_{2}^{2}\right)+\frac{X}{\phi}\right]\right\} \equiv \\
\equiv F E(\psi)
\end{gathered}
$$

however before to conclude we need to consider players' incentives with the other leader's strategies.

\subsection{The set of Sequential Equilibria}

1. if $(\zeta, \phi) \in\left[\frac{1}{X-\left(\Delta^{1}+\Delta^{2}\right)}, 1\right] \times[0,1]$ then in this region we get a unique equilibrium:

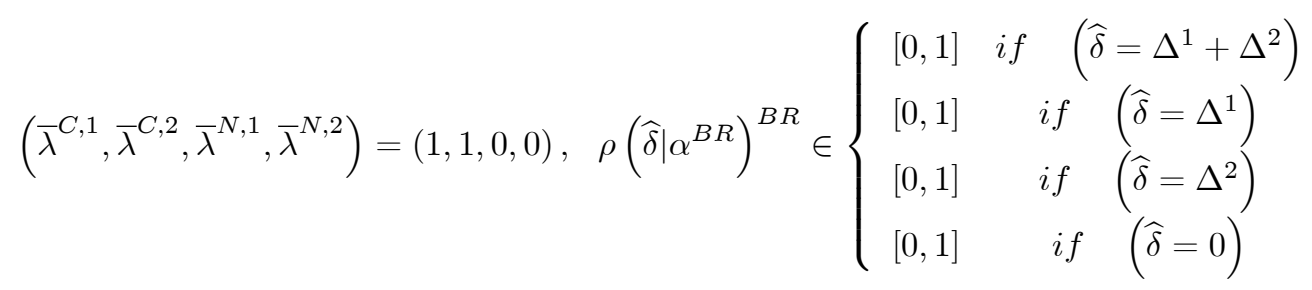




$$
\alpha(\widehat{\delta}, \widehat{\rho})^{B R}=\left\{\begin{array}{ccc}
1 & \text { if } & \left(\widehat{\delta}=\Delta^{1}+\Delta^{2}, \widehat{\rho}=0\right) \\
1 & \text { if } \quad\left(\widehat{\delta}=\Delta^{1}+\Delta^{2}, \widehat{\rho}=1\right) \\
1 & \text { if } \quad\left(\widehat{\delta}=\Delta^{1}, \widehat{\rho}=0\right) \\
1 & \text { if } \quad\left(\widehat{\delta}=\Delta^{1}, \widehat{\rho}=1\right) \\
1 & \text { if } \quad\left(\widehat{\delta}=\Delta^{2}, \widehat{\rho}=0\right) \\
1 & \text { if } \quad\left(\widehat{\delta}=\Delta^{2}, \widehat{\rho}=1\right) \\
1 & \text { if } \quad(\widehat{\delta}=0, \widehat{\rho}=0) \\
1 & \text { if } \quad(\widehat{\delta}=0, \widehat{\rho}=1)
\end{array}\right.
$$

2. if $(\zeta, \phi) \in\left[\frac{1}{X-\left(\pi\left(\Delta^{1}+\Delta^{2}\right)+(1-\pi) \psi \Delta^{2}\right)}, \frac{1}{X-\left(\Delta^{1}+\Delta^{2}\right)}\right] \times[0,1]$ then in this region we may get a mixed strategy equilibrium with:

$$
\left(\bar{\lambda}^{C, 1}, \bar{\lambda}^{C, 2}, \bar{\lambda}^{N, 1}, \bar{\lambda}^{N, 2}\right)=\left\{\begin{aligned}
(1,1,0,0) & \text { with probability } \operatorname{MFI}(\psi) \\
(1,1,1,0) & \text { with probability } \operatorname{MPE}(\psi) \\
(1,1,1,1) & \text { with probability } \operatorname{MFE}(\psi)
\end{aligned}\right.
$$

Let we find the properties of this mixed equilibrium.

The citizens Let consider the citizens' beliefs with the above leader's mixed strategy:

$$
\mu(C \mid \widehat{\delta})= \begin{cases}\frac{\pi}{\pi+(1-\pi)[M F E(\psi)+\psi M P E(\psi)]} & \widehat{\delta}=\Delta^{1}+\Delta^{2} \\ 0 & \widehat{\delta}=\Delta^{2} \\ 0 & \widehat{\delta}=\Delta^{1} \\ 0 & \widehat{\delta}=0\end{cases}
$$

Then, since

$$
\begin{gathered}
\alpha=1 \Leftrightarrow V^{Z}(\alpha=1 \mid \delta, \rho)=X-(\zeta)^{-1} \geq V^{Z}(\alpha=0 \mid \delta, \rho)=\mu(C \mid \delta, \rho)\left(\Delta^{1}+\Delta^{2}\right)+[1-\mu(C \mid \delta, \rho)] \psi \Delta^{2} \Leftrightarrow \\
\Leftrightarrow \alpha=1 \Leftrightarrow V^{Z}(\alpha=1 \mid \delta, \rho)=\mu(C \mid \delta, \rho) \leq \frac{X-(\zeta)^{-1}-\psi \Delta^{2}}{\Delta^{1}+\Delta^{2}-\psi \Delta^{2}} \Leftrightarrow \\
\Leftrightarrow \alpha \in\left\{\begin{array}{ccc}
\{1\} & \text { iff } & \frac{\pi}{\pi+(1-\pi)[M F E(\psi)+\psi M P E(\psi)]} \leq \frac{X-(\zeta)^{-1}-\psi \Delta^{2}}{\Delta^{1}+\Delta^{2}-\psi \Delta^{2}} \\
\in[0,1] & \text { iff } & \frac{\pi}{\pi+(1-\pi)[M F E(\psi)+\psi M P E(\psi)]}=\frac{X-(\zeta)-1}{\Delta^{1}+\Delta^{2}-\psi \Delta^{2}} \\
\{0\} & \text { iff } & \frac{\pi}{\pi+(1-\pi)[M F E(\psi)+\psi M P E(\psi)]} \geq \frac{X-(\zeta)^{-1}-\psi \Delta^{2}}{\Delta^{1}+\Delta^{2}-\psi \Delta^{2}}
\end{array}\right.
\end{gathered}
$$

however note that if $\alpha=1$, then $M F I=1$ however $\alpha=1$ requires $\frac{\pi}{\pi+(1-\pi)[M F E(\psi)+\psi M P E(\psi)]} \leq$ $\frac{X-(\zeta)^{-1}-\psi \Delta^{2}}{\Delta^{1}+\Delta^{2}-\psi \Delta^{2}} \Leftrightarrow \zeta \geq \frac{1}{X-\left(\Delta^{1}+\Delta^{2}\right)}$ which is not possible in this region; similarly if $\alpha=0$, then 
$M F I=1$ this again requires $\frac{\pi}{\pi+(1-\pi)[M F E(\psi)+\psi M P E(\psi)]} \leq \frac{X-(\zeta)^{-1}-\psi \Delta^{2}}{\Delta^{1}+\Delta^{2}-\psi \Delta^{2}} \Leftrightarrow \zeta \geq \frac{1}{X-\left(\Delta^{1}+\Delta^{2}\right)}$ which is not possible in this region. Hence to have a SE the citizens should choose

$$
\alpha(\widehat{\delta}, \widehat{\rho})^{B R}=\left\{\begin{array}{ccc}
\bar{\alpha} \in(0,1) \text { if } \quad\left(\widehat{\delta}=\Delta^{1}+\Delta^{2}, \widehat{\rho}=0\right) \\
1 & \text { if } \quad\left(\widehat{\delta}=\Delta^{1}+\Delta^{2}, \widehat{\rho}=1\right) \\
1 & \text { if } \quad\left(\widehat{\delta}=\Delta^{1}, \widehat{\rho}=0\right) \\
1 & \text { if } \quad\left(\widehat{\delta}=\Delta^{1}, \widehat{\rho}=1\right) \\
1 & \text { if } \quad\left(\widehat{\delta}=\Delta^{2}, \widehat{\rho}=0\right) \\
1 & \text { if } \quad\left(\widehat{\delta}=\Delta^{2}, \widehat{\rho}=1\right) \\
1 & \text { if } \quad(\widehat{\delta}=0, \widehat{\rho}=0) \\
1 & \text { if } \quad(\widehat{\delta}=0, \widehat{\rho}=1)
\end{array}\right.
$$

which requires

$$
\begin{aligned}
& \mu\left(C \mid \widehat{\delta}=\Delta^{1}+\Delta^{2}, \widehat{\rho}=1\right)=\frac{\pi}{\pi+(1-\pi)[M F E(\psi)+\psi M P E(\psi)]}=\frac{X-(\zeta)^{-1}-\psi \Delta^{2}}{\Delta^{1}+\Delta^{2}-\psi \Delta^{2}} \Leftrightarrow \\
& \Leftrightarrow \operatorname{MFE}(\psi)+\psi M P E(\psi)=\frac{\pi}{1-\pi} \frac{\Delta^{1}+\Delta^{2}-X+(\zeta)^{-1}}{X-(\zeta)^{-1}-\psi \Delta^{2}}=\frac{\pi}{1-\pi} \frac{1-\zeta\left(X-\left(\Delta^{1}+\Delta^{2}\right)\right)}{\zeta\left(X-\psi \Delta^{2}\right)-1}
\end{aligned}
$$

The Selectorate Suppose

$$
\begin{gathered}
\zeta \in\left[\frac{1}{X-\left[\pi\left(\Delta^{1}+\Delta^{2}\right)+(1-\pi) \psi \Delta^{2}\right]}, \frac{1}{X-\left(\Delta^{1}+\Delta^{2}\right)}\right] \\
\rho\left(\alpha^{B R} \mid \widehat{\delta}=\Delta^{1}+\Delta^{2}\right)=0 \Leftrightarrow V^{S}\left(\rho=0, \alpha^{B R} \mid \widehat{\delta}=\Delta^{1}+\Delta^{2}\right) \geq V^{S}\left(\rho=1, \alpha^{B R} \mid \widehat{\delta}=\Delta^{1}+\Delta^{2}\right) \Leftrightarrow \\
\Leftrightarrow(1-\bar{\alpha})\left[\frac{\pi}{\pi+(1-\pi)[M F E(\psi)+\psi M P E(\psi)]}+\left[1-\frac{\pi}{\pi+(1-\pi)[M F E(\psi)+\psi M P E(\psi)]}\right] \psi \Delta^{2}+\frac{X}{\phi}\right] \geq 0 \Leftrightarrow \\
\Leftrightarrow \rho\left(\widehat{\delta}=\Delta^{1}+\Delta^{2} \mid \alpha^{B R}\right)^{B R}=0
\end{gathered}
$$

Hence

$$
\rho\left(\widehat{\delta} \mid \alpha^{B R}\right)^{B R} \in\left\{\begin{array}{ccc}
\in\{0\} & \text { if } \quad\left(\widehat{\delta}=\Delta^{1}+\Delta^{2}\right) \\
{[0,1]} & \text { if }\left(\widehat{\delta}=\Delta^{1}\right) \\
{[0,1]} & \text { if }\left(\widehat{\delta}=\Delta^{2}\right) \\
{[0,1]} & \text { if } \quad(\widehat{\delta}=0)
\end{array} .\right.
$$

We can conclude this first part with following lemma

\section{Lemma 1 . When}

$$
\zeta \in\left[\frac{1}{X-\left[\pi\left(\Delta^{1}+\Delta^{2}\right)+(1-\pi) \psi \Delta^{2}\right]}, \frac{1}{X-\left(\Delta^{1}+\Delta^{2}\right)}\right]
$$


then

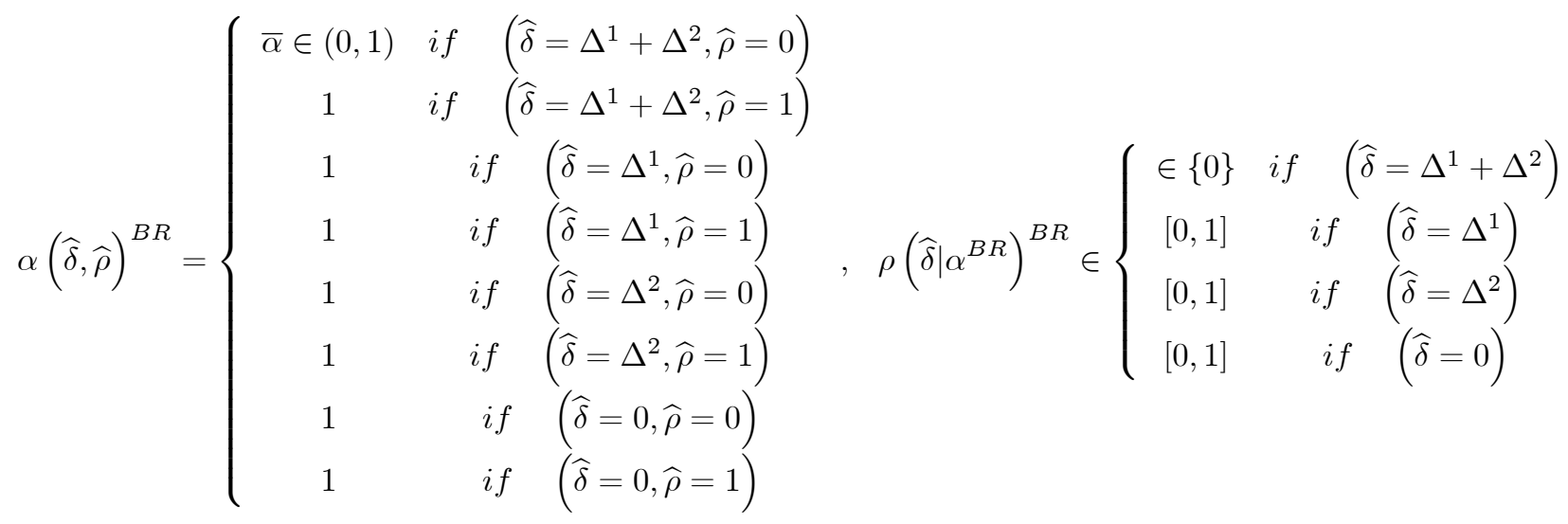

if and only if

$$
\left(\bar{\lambda}^{C, 1}, \bar{\lambda}^{C, 2}, \bar{\lambda}^{N, 1}, \bar{\lambda}^{N, 2}\right)=\left\{\begin{array}{l}
(1,1,0,0) \text { with probability } 1-\operatorname{MFE}(\psi)-\operatorname{MPE}(\psi) \\
(1,1,1,0) \text { with probability } \operatorname{MPE}(\psi) \\
(1,1,1,1) \text { with probability } \operatorname{MFE}(\psi)
\end{array}\right.
$$

where

$$
\operatorname{MFE}(\psi)+\psi M P E(\psi)=\frac{\pi}{1-\pi} \frac{\Delta^{1}+\Delta^{2}-X+(\zeta)^{-1}}{X-(\zeta)^{-1}-\psi \Delta^{2}}=\frac{\pi}{1-\pi} \frac{1-\zeta\left(X-\left(\Delta^{1}+\Delta^{2}\right)\right)}{\zeta\left(X-\psi \Delta^{2}\right)-1} .
$$

The Non Congruent Leader We have to check whether the strategy profile

$$
\left(\bar{\lambda}^{C, 1}, \bar{\lambda}^{C, 2}, \bar{\lambda}^{N, 1}, \bar{\lambda}^{N, 2}\right)= \begin{cases}(1,1,0,0) & \text { with probability } \operatorname{MFI}(\psi) \\ (1,1,1,0) & \text { with probability } \operatorname{MPE}(\psi) \\ (1,1,1,1) & \text { with probability } \operatorname{MFE}(\psi)\end{cases}
$$

is part of of a SE when

$$
(\zeta, \phi) \in\left[\frac{1}{X-\left(\pi\left(\Delta^{1}+\Delta^{2}\right)+(1-\pi) \psi \Delta^{2}\right)}, \frac{1}{X-\left(\Delta^{1}+\Delta^{2}\right)}\right] \times[0,1]
$$

Her payoff will depend on $\rho^{B R}$ and $\alpha^{B R}$, hence on the basis of the previous $\alpha(\widehat{\delta}, \widehat{\rho})^{B R}$ and $\rho\left(\widehat{\delta} \mid \alpha^{B R}\right)^{B R}$ Then with the conjectured SE s.t.

$$
\begin{gathered}
V^{N}\left(\bar{\lambda}^{C, 1}, \bar{\lambda}^{C, 2}, \bar{\lambda}^{N, 1}, \bar{\lambda}^{N, 2} ; \rho^{B R}, \alpha^{B R}\right)= \\
=\operatorname{MFI}\left(\psi \Delta^{2}+r_{1}^{1}+r_{1}^{2}+\frac{X}{\phi}\right)+M P E\left(\psi \Delta^{2}+\Delta^{1}+r_{1}^{2}+\frac{X}{\phi}+\psi(1-\bar{\alpha})\left[\psi \Delta^{2}+E\left(r_{2}^{1}\right)+E\left(r_{2}^{2}\right)+\frac{X}{\phi}\right]\right)+ \\
+\operatorname{MFE}\left(\Delta^{1}+\Delta^{2}+\frac{X}{\phi}+(1-\bar{\alpha})\left[\psi \Delta^{2}+E\left(r_{2}^{1}\right)+E\left(r_{2}^{2}\right)+\frac{X}{\phi}\right]\right)
\end{gathered}
$$


while deviating she get

$$
\begin{gathered}
V^{N}\left(\bar{\lambda}^{C, 1}, \bar{\lambda}^{C, 2}, \bar{\lambda}^{N, 1}, \bar{\lambda}^{N, 2} ; \rho^{B R}, \alpha^{B R}\right)= \\
= \begin{cases}\psi \Delta^{2}+r_{1}^{1}+r_{1}^{2}+\frac{X}{\phi}+0 & \text { if } \bar{\lambda}^{C, 1}, \bar{\lambda}^{C, 2}, \bar{\lambda}^{N, 1}, \bar{\lambda}^{N, 2}=1,1,0,0 \\
\Delta^{2}+r_{1}^{1}+\frac{X}{\phi}+0 & \text { if } \bar{\lambda}^{C, 1}, \bar{\lambda}^{C, 2}, \bar{\lambda}^{N, 1}, \bar{\lambda}^{N, 2}=1,1,0,1 \\
\Delta^{1}+\psi \Delta^{2}+r_{1}^{2}+\frac{X}{\phi}+\psi(1-\bar{\alpha})\left[\psi \Delta^{2}+E\left(r_{2}^{1}\right)+E\left(r_{2}^{2}\right)+\frac{X}{\phi}\right] & \text { if } \bar{\lambda}^{C, 1}, \bar{\lambda}^{C, 2}, \bar{\lambda}^{N, 1}, \bar{\lambda}^{N, 2}=1,1,1,0 \\
\Delta^{1}+\Delta^{2}+\frac{X}{\phi}+(1-\bar{\alpha})\left[\psi \Delta^{2}+E\left(r_{2}^{1}\right)+E\left(r_{2}^{2}\right)+\frac{X}{\phi}\right] & \text { if } \bar{\lambda}^{C, 1}, \bar{\lambda}^{C, 2}, \bar{\lambda}^{N, 1}, \bar{\lambda}^{N, 2}=1,1,1,1\end{cases}
\end{gathered}
$$

Hence its is an equilibrium if and only if $\bar{\alpha}$ is such that

$$
\left\{\begin{array}{c}
M F I\left(\psi \Delta^{2}+r_{1}^{1}+r_{1}^{2}+\frac{X}{\phi}\right)+M P E\left(\psi \Delta^{2}+\Delta^{1}+r_{1}^{2}+\frac{X}{\phi}+\psi(1-\bar{\alpha})\left[\psi \Delta^{2}+E\left(r_{2}^{1}\right)+E\left(r_{2}^{2}\right)+\frac{X}{\phi}\right]\right)+ \\
+M F E\left(\Delta^{1}+\Delta^{2}+\frac{X}{\phi}+(1-\bar{\alpha})\left[\psi \Delta^{2}+E\left(r_{2}^{1}\right)+E\left(r_{2}^{2}\right)+\frac{X}{\phi}\right]\right) \geq \psi \Delta^{2}+r_{1}^{1}+r_{1}^{2}+\frac{X}{\phi} \\
M F I\left(\psi \Delta^{2}+r_{1}^{1}+r_{1}^{2}+\frac{X}{\phi}\right)+M P E\left(\psi \Delta^{2}+\Delta^{1}+r_{1}^{2}+\frac{X}{\phi}+\psi(1-\bar{\alpha})\left[\psi \Delta^{2}+E\left(r_{2}^{1}\right)+E\left(r_{2}^{2}\right)+\frac{X}{\phi}\right]\right)+ \\
+M F E\left(\Delta^{1}+\Delta^{2}+\frac{X}{\phi}+(1-\bar{\alpha})\left[\psi \Delta^{2}+E\left(r_{2}^{1}\right)+E\left(r_{2}^{2}\right)+\frac{X}{\phi}\right]\right) \geq \\
\geq \Delta^{1}+\psi \Delta^{2}+r_{1}^{2}+\frac{X}{\phi}+\psi(1-\bar{\alpha})\left[\psi \Delta^{2}+E\left(r_{2}^{1}\right)+E\left(r_{2}^{2}\right)+\frac{X}{\phi}\right] \\
M F I\left(\psi \Delta^{2}+r_{1}^{1}+r_{1}^{2}+\frac{X}{\phi}\right)+M P E\left(\psi \Delta^{2}+\Delta^{1}+r_{1}^{2}+\frac{X}{\phi}+\psi(1-\bar{\alpha})\left[\psi \Delta^{2}+E\left(r_{2}^{1}\right)+E\left(r_{2}^{2}\right)+\frac{X}{\phi}\right]\right)+ \\
+M F E\left(\Delta^{1}+\Delta^{2}+\frac{X}{\phi}+(1-\bar{\alpha})\left[\psi \Delta^{2}+E\left(r_{2}^{1}\right)+E\left(r_{2}^{2}\right)+\frac{X}{\phi}\right]\right) \geq \\
\geq \Delta^{1}+\Delta^{2}+\frac{X}{\phi}+(1-\bar{\alpha})\left[\psi \Delta^{2}+E\left(r_{2}^{1}\right)+E\left(r_{2}^{2}\right)+\frac{X}{\phi}\right]
\end{array}\right.
$$

\section{Deviation to full inefficiency}

$$
\begin{gathered}
\Leftrightarrow \quad M F I\left(\psi \Delta^{2}+r_{1}^{1}+r_{1}^{2}+\frac{X}{\phi}\right)+\operatorname{MPE}\left(\psi \Delta^{2}+\Delta^{1}+r_{1}^{2}+\frac{X}{\phi}+\psi(1-\bar{\alpha})\left[\psi \Delta^{2}+E\left(r_{2}^{1}\right)+E\left(r_{2}^{2}\right)+\frac{X}{\phi}\right]\right)+ \\
\quad+M F E\left(\Delta^{1}+\Delta^{2}+\frac{X}{\phi}+(1-\bar{\alpha})\left[\psi \Delta^{2}+E\left(r_{2}^{1}\right)+E\left(r_{2}^{2}\right)+\frac{X}{\phi}\right]\right) \leq \psi \Delta^{2}+r_{1}^{1}+r_{1}^{2}+\frac{X}{\phi} \Leftrightarrow \\
\Leftrightarrow M F I=\mathbf{P}\left\{(1-M F I) r_{1}^{1}+M F E r_{1}^{2} \geq-(1-M F I)(A)+M F E(B+(1-\bar{\alpha})[C])+M P E(D+\psi(1-\bar{\alpha})[C])\right\}
\end{gathered}
$$

where

$$
\left\{\begin{array}{c}
A \equiv \psi \Delta^{2}+\frac{X}{\phi} \\
B \equiv \Delta^{1}+\Delta^{2}+\frac{X}{\phi} \\
C \equiv \psi \Delta^{2}+E\left(r_{2}^{1}\right)+E\left(r_{2}^{2}\right)+\frac{X}{\phi} \\
D \equiv \Delta^{1}+\psi \Delta^{2}+\frac{X}{\phi}
\end{array}\right.
$$

\section{Deviation to Partial Inefficiency}

$$
\begin{aligned}
& M F I\left(\psi \Delta^{2}+r_{1}^{1}+r_{1}^{2}+\frac{X}{\phi}\right)+\operatorname{MPE}\left(\psi \Delta^{2}+\Delta^{1}+r_{1}^{2}+\frac{X}{\phi}+\psi(1-\bar{\alpha})\left[\psi \Delta^{2}+E\left(r_{2}^{1}\right)+E\left(r_{2}^{2}\right)+\frac{X}{\phi}\right]\right)+ \\
& +\operatorname{MFE}\left(\Delta^{1}+\Delta^{2}+\frac{X}{\phi}+(1-\bar{\alpha})\left[\psi \Delta^{2}+E\left(r_{2}^{1}\right)+E\left(r_{2}^{2}\right)+\frac{X}{\phi}\right]\right) \leq \\
\leq & \Delta^{1}+\psi \Delta^{2}+r_{1}^{2}+\frac{X}{\phi}+\psi(1-\bar{\alpha})\left[\psi \Delta^{2}+E\left(r_{2}^{1}\right)+E\left(r_{2}^{2}\right)+\frac{X}{\phi}\right] \Leftrightarrow
\end{aligned}
$$


$\Leftrightarrow M P E=\mathbf{P}\left\{M F I r_{1}^{1}-M F E r_{1}^{2} \leq-M F I(A)+M F E(B+(1-\bar{\alpha})[C])+(1-M P E)(D+\psi(1-\bar{\alpha})[C])\right\}$

\section{Deviation to Full Efficiency}

$$
\begin{aligned}
& M F I\left(\psi \Delta^{2}+r_{1}^{1}+r_{1}^{2}+\frac{X}{\phi}\right)+M P E\left(\psi \Delta^{2}+\Delta^{1}+r_{1}^{2}+\frac{X}{\phi}+\psi(1-\bar{\alpha})\left[\psi \Delta^{2}+E\left(r_{2}^{1}\right)+E\left(r_{2}^{2}\right)+\frac{X}{\phi}\right]\right)+ \\
& +M F E\left(\Delta^{1}+\Delta^{2}+\frac{X}{\phi}+(1-\bar{\alpha})\left[\psi \Delta^{2}+E\left(r_{2}^{1}\right)+E\left(r_{2}^{2}\right)+\frac{X}{\phi}\right]\right) \leq \\
\leq & \Delta^{1}+\Delta^{2}+\frac{X}{\phi}+(1-\bar{\alpha})\left[\psi \Delta^{2}+E\left(r_{2}^{1}\right)+E\left(r_{2}^{2}\right)+\frac{X}{\phi}\right] \Leftrightarrow \\
\Leftrightarrow & M F E=\mathbf{P}\left\{M F I r_{1}^{1}+(M F I+M F E) r_{1}^{2} \leq-M F I(A)+(1-M F E)(B+(1-\bar{\alpha})[C])-M P E(D+\psi(1-\bar{\alpha})[\right.
\end{aligned}
$$

From simulations, it is possible to check that the set of $\bar{\alpha} \in(0,1)$ satisfying the above conditions is not empty and monotonically shifting towards 1 as $\psi$ increases.

Now we can conclude with the following result:

Lemma 2. When

$$
\zeta \in\left[\frac{1}{X-\left[\pi\left(\Delta^{1}+\Delta^{2}\right)+(1-\pi) \psi \Delta^{2}\right]}, \frac{1}{X-\left(\Delta^{1}+\Delta^{2}\right)}\right]
$$

then

$$
\begin{aligned}
& \alpha(\widehat{\delta}, \widehat{\rho})^{B R}=\left\{\begin{array}{ccc}
\bar{\alpha} \in(0,1) & \text { if } & \left(\widehat{\delta}=\Delta^{1}+\Delta^{2}, \widehat{\rho}=0\right) \\
1 & \text { if } \quad\left(\widehat{\delta}=\Delta^{1}+\Delta^{2}, \widehat{\rho}=1\right) \\
1 & \text { if } \quad\left(\widehat{\delta}=\Delta^{1}, \widehat{\rho}=0\right) \\
1 & \text { if } & \left(\widehat{\delta}=\Delta^{1}, \widehat{\rho}=1\right) \\
1 & \text { if } & \left(\widehat{\delta}=\Delta^{2}, \widehat{\rho}=0\right) \\
1 & \text { if } & \left(\widehat{\delta}=\Delta^{2}, \widehat{\rho}=1\right) \\
1 & \text { if } & (\widehat{\delta}=0, \widehat{\rho}=0) \\
1 & \text { if } & (\widehat{\delta}=0, \widehat{\rho}=1)
\end{array}\right. \\
& \rho\left(\widehat{\delta} \mid \alpha^{B R}\right)^{B R} \in\left\{\begin{array}{cc}
\in\{0\} & \text { if } \quad\left(\widehat{\delta}=\Delta^{1}+\Delta^{2}\right) \\
{[0,1]} & \text { if }\left(\widehat{\delta}=\Delta^{1}\right) \\
{[0,1]} & \text { if } \quad\left(\widehat{\delta}=\Delta^{2}\right) \\
{[0,1]} & \text { if } \quad(\widehat{\delta}=0)
\end{array}\right. \\
& \left(\bar{\lambda}^{C, 1}, \bar{\lambda}^{C, 2}, \bar{\lambda}^{N, 1}, \bar{\lambda}^{N, 2}\right)=\left\{\begin{array}{l}
(1,1,0,0) \text { with probability } \operatorname{MFI}(\psi) \\
(1,1,1,0) \text { with probability } \operatorname{MPE}(\psi) \\
(1,1,1,1) \text { with probability } \operatorname{MFE}(\psi)
\end{array}\right.
\end{aligned}
$$




$$
\mu(C \mid \widehat{\delta})= \begin{cases}\frac{\pi}{\pi+(1-\pi)[M F E(\psi)+\psi M P E(\psi)]} & \widehat{\delta}=\Delta^{1}+\Delta^{2} \\ 0 & \widehat{\delta}=\Delta^{2} \\ 0 & \widehat{\delta}=\Delta^{1} \\ 0 & \widehat{\delta}=0\end{cases}
$$

is an equilibrium if and only if

$$
M F E+\psi M P E=\frac{\pi}{1-\pi} \frac{\Delta^{1}+\Delta^{2}-X+(\zeta)^{-1}}{X-(\zeta)^{-1}-\psi \Delta^{2}}=\frac{\pi}{1-\pi} \frac{1-\zeta\left(X-\left(\Delta^{1}+\Delta^{2}\right)\right)}{\zeta\left(X-\psi \Delta^{2}\right)-1}
$$

and

$M F I=\mathbf{P}\left\{(1-M F I) r_{1}^{1}+M F E r_{1}^{2} \geq-(1-M F I)(A)+M F E(B+(1-\bar{\alpha})[C])+M P E(D+\psi(1-\bar{\alpha})[C])\right\}$

$M P E=\mathbf{P}\left\{M F I r_{1}^{1}-M F E r_{1}^{2} \leq-M F I(A)+M F E(B+(1-\bar{\alpha})[C])+(1-M P E)(D+\psi(1-\bar{\alpha})[C])\right\}$

$M F E=\mathbf{P}\left\{M F I r_{1}^{1}+(M F I+M F E) r_{1}^{2} \leq-M F I(A)+(1-M F E)(B+(1-\bar{\alpha})[C])-M P E(D+\psi(1-\bar{\alpha})[C])\right.$

where

$$
\left\{\begin{array}{c}
A \equiv \psi \Delta^{2}+\frac{X}{\phi} \\
B \equiv \Delta^{1}+\Delta^{2}+\frac{X}{\phi} \\
C \equiv \psi \Delta^{2}+E\left(r_{2}^{1}\right)+E\left(r_{2}^{2}\right)+\frac{X}{\phi} \\
D \equiv \Delta^{1}+\psi \Delta^{2}+\frac{X}{\phi}
\end{array}\right.
$$

Thus the outcome in this region is a mixed strategy equilibrium with possible full inefficient policy, partially and full efficient policy, depending on the above probabilities. Moreover we have coups and revolution with strictly positive probability.

3. if $(\zeta, \phi) \in\left[\frac{1}{X-\psi \Delta^{2}}, \frac{1}{X-\left[\pi\left(\Delta^{1}+\Delta^{2}\right)+(1-\pi) \psi \Delta^{2}\right]}\right] \times[0,1]$ then in this region we get an equilibrium:

$$
\left(\bar{\lambda}^{C, 1}, \bar{\lambda}^{C, 2}, \bar{\lambda}^{N, 1}, \bar{\lambda}^{N, 2}\right)=(1,1,0,0)
$$

with probability

$$
\begin{gathered}
\mathbf{P}\left\{r_{1}^{1} \geq \Delta^{1}+\psi\left[\psi \Delta^{2}+E\left(r_{2}^{1}\right)+E\left(r_{2}^{2}\right)+\frac{X}{\phi}\right] \& r_{1}^{1}+r_{1}^{2} \geq \Delta^{2}+\Delta^{1}+E\left(r_{2}^{1}\right)+E\left(r_{2}^{2}\right)+\frac{X}{\phi}\right\}=F I(\psi) \\
\left(\bar{\lambda}^{C, 1}, \bar{\lambda}^{C, 2}, \bar{\lambda}^{N, 1}, \bar{\lambda}^{N, 2}\right)=(1,1,1,0)
\end{gathered}
$$

with probability

$$
\begin{aligned}
& \mathbf{P}\left\{r_{1}^{1} \leq \Delta^{1}+\psi\left[\psi \Delta^{2}+E\left(r_{2}^{1}\right)+E\left(r_{2}^{2}\right)+\frac{X}{\phi}\right] \& r_{1}^{2} \geq(1-\psi) \Delta^{2}+(1-\psi)\left[\psi \Delta^{2}+E\left(r_{2}^{1}\right)+E\left(r_{2}^{2}\right)+\frac{X}{\phi}\right]\right\} \\
& =G_{1}\left(\Delta^{1}+\psi\left[\psi \Delta^{2}+E\left(r_{2}^{1}\right)+E\left(r_{2}^{2}\right)+\frac{X}{\phi}\right]\right)\left[1-G_{2}\left((1-\psi)\left[\Delta^{2}+\psi \Delta^{2}+E\left(r_{2}^{1}\right)+E\left(r_{2}^{2}\right)+\frac{X}{\phi}\right]\right)\right]=
\end{aligned}
$$




$$
\begin{gathered}
=P E(\psi) \\
\left(\bar{\lambda}^{C, 1}, \bar{\lambda}^{C, 2}, \bar{\lambda}^{N, 1}, \bar{\lambda}^{N, 2}\right)=(1,1,1,1)
\end{gathered}
$$

with probability

$$
\begin{aligned}
& \mathbf{P}\left\{r_{1}^{1}+r_{1}^{2} \leq \Delta^{2}+\Delta^{1}+E\left(r_{2}^{1}\right)+E\left(r_{2}^{2}\right)+\frac{X}{\phi} \& r_{1}^{2} \leq(1-\psi) \Delta^{2}+(1-\psi)\left[\psi \Delta^{2}+E\left(r_{2}^{1}\right)+E\left(r_{2}^{2}\right)+\frac{X}{\phi}\right]\right\}= \\
& =F E(\psi)
\end{aligned}
$$

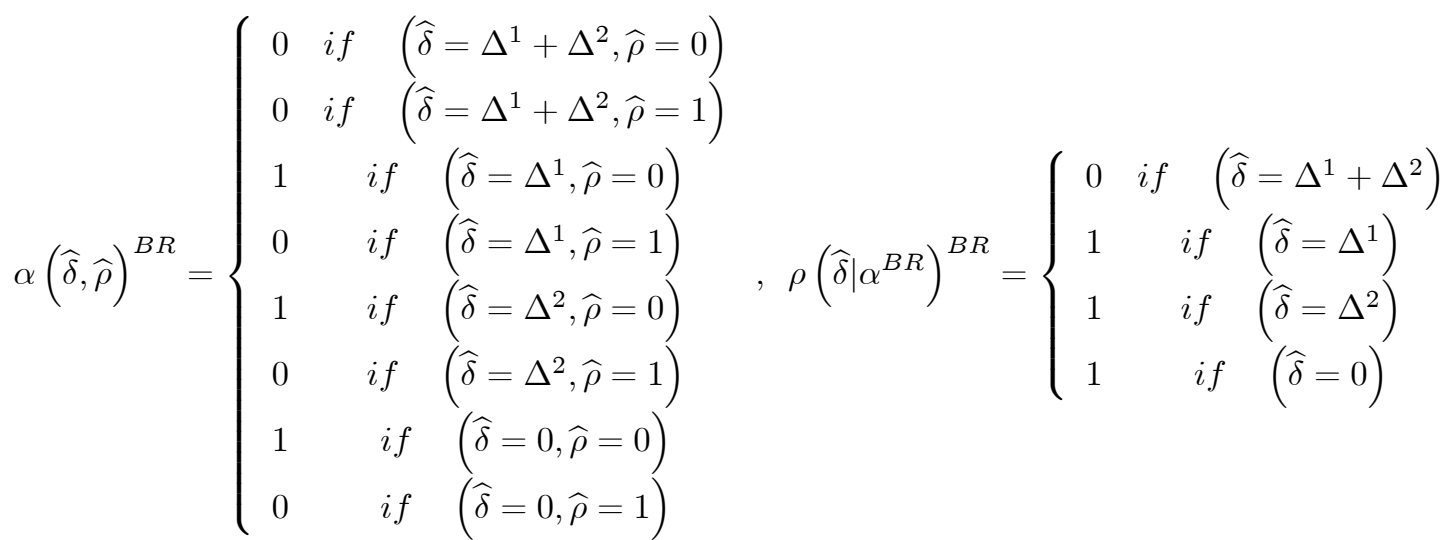

4. $(\zeta, \phi) \in\left[0, \frac{1}{X-\psi \Delta^{2}}\right] \times\left[0, \frac{X}{\pi\left(\Delta^{1}+\Delta^{2}-\psi \Delta^{2}\right)+X}\right]$ in this region we have a unique equilibrium:

$$
\alpha(\widehat{\delta}, \widehat{\rho})^{B R}=\left\{\begin{array}{l}
\left(\bar{\lambda}^{C, 1}, \bar{\lambda}^{C, 2}, \bar{\lambda}^{N, 1}, \bar{\lambda}^{N, 2}\right)=(1,1,0,0) \\
0 \text { if }\left(\widehat{\delta}=\Delta^{1}+\Delta^{2}, \widehat{\rho}=0\right) \\
0 \quad \text { if } \quad\left(\widehat{\delta}=\Delta^{1}+\Delta^{2}, \widehat{\rho}=1\right) \\
0 \quad \text { if } \quad\left(\widehat{\delta}=\Delta^{1}, \widehat{\rho}=0\right) \\
0 \quad \text { if } \quad\left(\widehat{\delta}=\Delta^{1}, \widehat{\rho}=1\right) \\
0 \quad \text { if } \quad\left(\widehat{\delta}=\Delta^{2}, \widehat{\rho}=0\right) \\
0 \quad \text { if } \quad\left(\widehat{\delta}=\Delta^{2}, \widehat{\rho}=1\right) \\
0 \quad \text { if } \quad(\widehat{\delta}=0, \widehat{\rho}=0) \\
0 \quad \text { if } \quad(\widehat{\delta}=0, \widehat{\rho}=1)
\end{array} \quad, \quad \rho\left(\widehat{\delta} \mid \alpha^{B R}\right)^{B R}=\left\{\begin{array}{cc}
0 & \text { if } \quad\left(\widehat{\delta}=\Delta^{1}+\Delta^{2}\right) \\
0 & \text { if } \quad\left(\widehat{\delta}=\Delta^{1}\right) \\
0 & \text { if } \quad\left(\widehat{\delta}=\Delta^{2}\right) \\
0 & \text { if } \quad(\widehat{\delta}=0)
\end{array}\right.\right.
$$

5. $(\zeta, \phi) \in\left[0, \frac{1}{X-\psi \Delta^{2}}\right] \times\left[\frac{X}{\pi\left(\Delta^{1}+\Delta^{2}\right)+X}, 1\right]$ in this region we get an equilibrium:

$$
\left(\bar{\lambda}^{C, 1}, \bar{\lambda}^{C, 2}, \bar{\lambda}^{N, 1}, \bar{\lambda}^{N, 2}\right)=(1,1,0,0)
$$

with probability

$$
F I(\psi)=\mathbf{P}\left\{r_{1}^{1} \geq \Delta^{1}+\psi\left[\psi \Delta^{2}+E\left(r_{2}^{1}\right)+E\left(r_{2}^{2}\right)+\frac{X}{\phi}\right] \& r_{1}^{1}+r_{1}^{2} \geq \Delta^{2}+\Delta^{1}+E\left(r_{2}^{1}\right)+E\left(r_{2}^{2}\right)+\frac{X}{\phi}\right\}
$$




$$
\left(\bar{\lambda}^{C, 1}, \bar{\lambda}^{C, 2}, \bar{\lambda}^{N, 1}, \bar{\lambda}^{N, 2}\right)=(1,1,1,0)
$$

with probability

$$
\begin{gathered}
\mathbf{P}\left\{r_{1}^{1} \leq \Delta^{1}+\psi\left[\psi \Delta^{2}+E\left(r_{2}^{1}\right)+E\left(r_{2}^{2}\right)+\frac{X}{\phi}\right] \& r_{1}^{2} \geq(1-\psi) \Delta^{2}+(1-\psi)\left[\psi \Delta^{2}+E\left(r_{2}^{1}\right)+E\left(r_{2}^{2}\right)+\frac{X}{\phi}\right]\right\}= \\
=G_{1}\left(\Delta^{1}+\psi\left[\psi \Delta^{2}+E\left(r_{2}^{1}\right)+E\left(r_{2}^{2}\right)+\frac{X}{\phi}\right]\right)\left[1-G_{2}\left((1-\psi)\left[\Delta^{2}+\psi \Delta^{2}+E\left(r_{2}^{1}\right)+E\left(r_{2}^{2}\right)+\frac{X}{\phi}\right]\right)\right]= \\
=P E(\psi)
\end{gathered}
$$

$$
\alpha(\widehat{\delta}, \widehat{\rho})^{B R}=\left\{\begin{array}{l}
\left(\bar{\lambda}^{C, 1}, \bar{\lambda}^{C, 2}, \bar{\lambda}^{N, 1}, \bar{\lambda}^{N, 2}\right)=(1,1,1,1) \\
0 \text { if }\left(\widehat{\delta}=\Delta^{1}+\Delta^{2}, \widehat{\rho}=0\right) \\
0 \quad \text { if }\left(\widehat{\delta}=\Delta^{1}+\Delta^{2}, \widehat{\rho}=1\right) \\
0 \quad \text { if } \quad\left(\widehat{\delta}=\Delta^{1}, \widehat{\rho}=0\right) \\
0 \quad \text { if } \quad\left(\widehat{\delta}=\Delta^{1}, \widehat{\rho}=1\right) \\
0 \quad \text { if } \quad\left(\widehat{\delta}=\Delta^{2}, \widehat{\rho}=0\right) \\
0 \quad \text { if } \quad\left(\widehat{\delta}=\Delta^{2}, \widehat{\rho}=1\right) \\
0 \quad \text { if }(\widehat{\delta}=0, \widehat{\rho}=0) \\
0 \quad \text { if } \quad(\widehat{\delta}=0, \widehat{\rho}=1)
\end{array} \quad, \rho\left(\widehat{\delta} \mid \alpha^{B R}\right)^{B R}=\left\{\begin{array}{cc}
0 & \text { if } \quad\left(\widehat{\delta}=\Delta^{1}+\Delta^{2}\right) \\
1 & \text { if } \quad\left(\widehat{\delta}=\Delta^{1}\right) \\
1 & \text { if } \quad\left(\widehat{\delta}=\Delta^{2}\right) \\
1 & \text { if } \quad(\widehat{\delta}=0) \\
&
\end{array}\right.\right.
$$

with probability

$$
\begin{gathered}
\mathbf{P}\left\{r_{1}^{1}+r_{1}^{2} \leq \Delta^{2}+\Delta^{1}+E\left(r_{2}^{1}\right)+E\left(r_{2}^{2}\right)+\frac{X}{\phi} \& r_{1}^{2} \leq(1-\psi) \Delta^{2}+(1-\psi)\left[\psi \Delta^{2}+E\left(r_{2}^{1}\right)+E\left(r_{2}^{2}\right)+\frac{X}{\phi}\right]\right\}= \\
=F E(\psi) .
\end{gathered}
$$

We can sum up all these results in the following proposition

Proposition 1. 1. if $(\zeta, \phi) \in\left[\frac{1}{X-\left(\Delta^{1}+\Delta^{2}\right)}, 1\right] \times[0,1]$ then there is a unique Sequential

Equilibrium such that:

$$
\begin{gathered}
\left(\bar{\lambda}_{1}^{C, 1}, \bar{\lambda}_{1}^{C, 2}, \bar{\lambda}_{1}^{N, 1}, \bar{\lambda}_{1}^{N, 2}\right)=(1,1,0,0) \\
\rho(\widehat{\delta}) \in\left\{\begin{array}{lcc}
{[0,1]} & \text { if } \quad\left(\widehat{\delta}=\Delta^{1}+\Delta^{2}\right) \\
{[0,1]} & \text { if } & \left(\widehat{\delta}=\Delta^{1}\right) \\
{[0,1]} & \text { if } & \left(\widehat{\delta}=\Delta^{2}\right) \\
{[0,1]} & \text { if } & (\widehat{\delta}=0)
\end{array}\right.
\end{gathered}
$$




$$
\begin{gathered}
\alpha(\widehat{\delta}, \widehat{\rho})=\left\{\begin{array}{ccc}
1 & \text { if } & \left(\widehat{\delta}=\Delta^{1}+\Delta^{2}, \widehat{\rho}=0\right) \\
1 & \text { if } \quad\left(\widehat{\delta}=\Delta^{1}+\Delta^{2}, \widehat{\rho}=1\right) \\
1 & \text { if } & \left(\widehat{\delta}=\Delta^{1}, \widehat{\rho}=0\right) \\
1 & \text { if } & \left(\widehat{\delta}=\Delta^{1}, \widehat{\rho}=1\right) \\
1 & \text { if } & \left(\widehat{\delta}=\Delta^{2}, \widehat{\rho}=0\right) \\
1 & \text { if } & \left(\widehat{\delta}=\Delta^{2}, \widehat{\rho}=1\right) \\
1 & \text { if } & (\widehat{\delta}=0, \widehat{\rho}=0) \\
1 & \text { if } & (\widehat{\delta}=0, \widehat{\rho}=1)
\end{array}\right. \\
\mu(C \mid \widehat{\delta})= \begin{cases}1 & \widehat{\delta}=\Delta^{1}+\Delta^{2} \\
0 & \widehat{\delta}=\Delta^{2} \\
\frac{0}{0} \in[0,1] & \widehat{\delta}=\Delta^{1} \\
0 & \widehat{\delta}=0\end{cases} \\
\left(\bar{\lambda}_{2}^{C, 1}, \bar{\lambda}_{2}^{C, 2}, \bar{\lambda}_{2}^{N, 1}, \bar{\lambda}_{2}^{N, 2}\right)=(1,1,0,0)
\end{gathered}
$$

The outcome in this region is full inefficient policy, selectorate's coup with positive probability and citizens' revolution with certainty, exactly as in the unidimensional model.

2. if $(\zeta, \phi) \in\left[\frac{1}{X-\left(\pi\left(\Delta^{1}+\Delta^{2}\right)+(1-\pi) \psi \Delta^{2}\right)}, \frac{1}{X-\left(\Delta^{1}+\Delta^{2}\right)}\right] \times[0,1]$ then with FI there is a mixed strategy Sequential Equilibrium such that

$$
\begin{array}{r}
\left(\bar{\lambda}^{C, 1}, \bar{\lambda}^{C, 2}, \bar{\lambda}^{N, 1}, \bar{\lambda}^{N, 2}\right)= \begin{cases}(1,1,0,0) & \text { with probability } \operatorname{MFI}(\psi) \\
(1,1,1,0) & \text { with probability } \operatorname{MPE}(\psi) \\
(1,1,1,1) & \text { with probability MFE }(\psi)\end{cases} \\
\rho\left(\widehat{\delta} \mid \alpha^{B R}\right)^{B R} \in\left\{\begin{array}{lll}
\in\{0\} & \text { if } \quad\left(\widehat{\delta}=\Delta^{1}+\Delta^{2}\right) \\
{[0,1]} & \text { if }\left(\widehat{\delta}=\Delta^{1}\right) \\
{[0,1]} & \text { if }\left(\widehat{\delta}=\Delta^{2}\right) \\
{[0,1]} & \text { if } \quad(\widehat{\delta}=0)
\end{array}\right.
\end{array}
$$




$$
\begin{aligned}
& \alpha(\widehat{\delta}, \widehat{\rho})^{B R}=\left\{\begin{array}{ccc}
\bar{\alpha} \in(0,1) & \text { if } \quad\left(\widehat{\delta}=\Delta^{1}+\Delta^{2}, \widehat{\rho}=0\right) \\
1 & \text { if } \quad\left(\widehat{\delta}=\Delta^{1}+\Delta^{2}, \widehat{\rho}=1\right) \\
1 & \text { if } \quad\left(\widehat{\delta}=\Delta^{1}, \widehat{\rho}=0\right) \\
1 & \text { if } \quad\left(\widehat{\delta}=\Delta^{1}, \widehat{\rho}=1\right) \\
1 & \text { if } \quad\left(\widehat{\delta}=\Delta^{2}, \widehat{\rho}=0\right) \\
1 & \text { if } \quad\left(\widehat{\delta}=\Delta^{2}, \widehat{\rho}=1\right) \\
1 & \text { if } \quad(\widehat{\delta}=0, \widehat{\rho}=0) \\
1 & \text { if } \quad(\widehat{\delta}=0, \widehat{\rho}=1)
\end{array}\right. \\
& \mu(C \mid \widehat{\delta})= \begin{cases}\frac{\pi}{\pi+(1-\pi)[M F E(\psi)+\psi M P E(\psi)]} & \widehat{\delta}=\Delta^{1}+\Delta^{2} \\
0 & \widehat{\delta}=\Delta^{2} \\
0 & \widehat{\delta}=\Delta^{1} \\
0 & \widehat{\delta}=0\end{cases}
\end{aligned}
$$

such that

$$
M F E+\psi M P E=\frac{\pi}{1-\pi} \frac{\Delta^{1}+\Delta^{2}-X+(\zeta)^{-1}}{X-(\zeta)^{-1}-\psi \Delta^{2}}=\frac{\pi}{1-\pi} \frac{1-\zeta\left(X-\left(\Delta^{1}+\Delta^{2}\right)\right)}{\zeta\left(X-\psi \Delta^{2}\right)-1}
$$

and

$$
\begin{gathered}
M F I=\mathbf{P}\left\{(1-M F I) r_{1}^{1}+M F E r_{1}^{2} \geq-(1-M F I)(A)+M F E(B+(1-\bar{\alpha})[C])+M P E(D+\psi(1-\bar{\alpha})[C\right. \\
M P E=\mathbf{P}\left\{M F I r_{1}^{1}-M F E r_{1}^{2} \leq-M F I(A)+M F E(B+(1-\bar{\alpha})[C])+(1-M P E)(D+\psi(1-\bar{\alpha})[C])\right\} \\
M F E= \\
=\mathbf{P}\left\{M F I r_{1}^{1}+(M F I+M F E) r_{1}^{2} \leq-M F I(A)+(1-M F E)(B+(1-\bar{\alpha})[C])-M P E(D+\psi(1-\bar{\alpha})[C])\right\}
\end{gathered}
$$

where

$$
\begin{gathered}
\left\{\begin{array}{c}
A \equiv \psi \Delta^{2}+\frac{X}{\phi} \\
B \equiv \Delta^{1}+\Delta^{2}+\frac{X}{\phi} \\
C \equiv \psi \Delta^{2}+E\left(r_{2}^{1}\right)+E\left(r_{2}^{2}\right)+\frac{X}{\phi} \\
D \equiv \Delta^{1}+\psi \Delta^{2}+\frac{X}{\phi}
\end{array}\right. \\
\left(\bar{\lambda}_{2}^{C, 1}, \bar{\lambda}_{2}^{C, 2}, \bar{\lambda}_{2}^{N, 1}, \bar{\lambda}_{2}^{N, 2}\right)=(1,1,0,0)
\end{gathered}
$$

The outcome in this region is a mixed strategy equilibrium with possible full inefficient policy, partially and full efficient policy, depending on the above probabilities. Moreover we have coups and revolution with strictly positive probability. 
3. if $(\zeta, \phi) \in\left[\frac{1}{X-\psi \Delta^{2}}, \frac{1}{X-\left[\pi\left(\Delta^{1}+\Delta^{2}\right)+(1-\pi) \psi \Delta^{2}\right]}\right] \times[0,1]$ then with FI there is a unique Sequential Equilibrium such that

$$
\left(\bar{\lambda}_{1}^{C, 1}, \bar{\lambda}_{1}^{C, 2}, \bar{\lambda}_{1}^{N, 1}, \bar{\lambda}_{1}^{N, 2}\right)=\left\{\begin{array}{l}
(1,1,0,0) \\
(1,1,1,0) \text { with prob. } F I(\psi) \\
(1,1,1,1) \text { with prob. } \operatorname{PE}(\psi)
\end{array}\right.
$$

where

$i$.

$$
\begin{gathered}
F I(\psi)= \\
=\mathbf{P}\left\{r_{1}^{1} \geq \Delta^{1}+\psi\left[\psi \Delta^{2}+E\left(r_{2}^{1}\right)+E\left(r_{2}^{2}\right)+\frac{X}{\phi}\right] \& r_{1}^{1}+r_{1}^{2} \geq \Delta^{2}+\Delta^{1}+E\left(r_{2}^{1}\right)+E\left(r_{2}^{2}\right)+\frac{X}{\phi}\right\}
\end{gathered}
$$

ii.

$$
\begin{gathered}
P E(\psi)= \\
\mathbf{P}\left\{r_{1}^{1} \leq \Delta^{1}+\psi\left[\psi \Delta^{2}+E\left(r_{2}^{1}\right)+E\left(r_{2}^{2}\right)+\frac{X}{\phi}\right] \& r_{1}^{2} \geq(1-\psi) \Delta^{2}+(1-\psi)\left[\psi \Delta^{2}+E\left(r_{2}^{1}\right)+E\left(r_{2}^{2}\right)+\frac{X}{\phi}\right.\right. \\
=G_{1}\left(\Delta^{1}+\psi\left[\psi \Delta^{2}+E\left(r_{2}^{1}\right)+E\left(r_{2}^{2}\right)+\frac{X}{\phi}\right]\right)\left[1-G_{2}\left((1-\psi)\left[\Delta^{2}+\psi \Delta^{2}+E\left(r_{2}^{1}\right)+E\left(r_{2}^{2}\right)+\frac{X}{\phi}\right]\right)\right]
\end{gathered}
$$

iii.

$$
\begin{gathered}
F E(\psi)= \\
=\mathbf{P}\left\{r_{1}^{1}+r_{1}^{2} \leq \Delta^{2}+\Delta^{1}+E\left(r_{2}^{1}\right)+E\left(r_{2}^{2}\right)+\frac{X}{\phi} \& r_{1}^{2} \leq(1-\psi) \Delta^{2}+(1-\psi)\left[\psi \Delta^{2}+E\left(r_{2}^{1}\right)+E\left(r_{2}^{2}\right)+\frac{X}{\phi}\right.\right. \\
\alpha(\widehat{\delta}, \widehat{\rho})= \begin{cases}0 & \text { if }\left(\widehat{\delta}=\Delta^{1}+\Delta^{2}\right) \\
1 & \text { if }\left(\widehat{\delta}=\Delta^{1}\right) \\
1 & \text { if }\left(\widehat{\delta}=\Delta^{2}\right) \\
1 & \text { if }(\widehat{\delta}=0) \\
1 & \text { if } \quad\left(\widehat{\delta}=\Delta^{2}, \widehat{\rho}=0\right) \\
1 & \text { if }\left(\widehat{\delta}=\Delta^{1}, \widehat{\rho}=0\right) \\
0 & \text { if } \quad\left(\widehat{\delta}=\Delta^{2}, \widehat{\rho}=1\right) \\
1 & \text { if }(\widehat{\delta}=0, \widehat{\rho}=0) \\
0 & \text { if }(\widehat{\delta}=0, \widehat{\rho}=1)\end{cases}
\end{gathered}
$$




$$
\begin{aligned}
\mu(C \mid \widehat{\delta})= & \begin{cases}\frac{\pi}{\pi+(1-\pi)[F E(\psi)+\psi P E(\psi)]} & \widehat{\delta}=\Delta^{1}+\Delta^{2} \\
0 & \widehat{\delta}=\Delta^{2} \\
0 & \widehat{\delta}=\Delta^{1} \\
0 & \widehat{\delta}=0\end{cases} \\
& \left(\bar{\lambda}_{2}^{C, 1}, \bar{\lambda}_{2}^{C, 2}, \bar{\lambda}_{2}^{N, 1}, \bar{\lambda}_{2}^{N, 2}\right)=(1,1,0,0)
\end{aligned}
$$

The outcome in this region is a mixed strategy equilibrium with possible full inefficient, partially and full efficient policy, depending on the probabilities $F I(\psi), P E(\psi)$ and $F E(\psi)$. Moreover we have coups with strictly positive probability, but no revolution in equilibrium.

4. $(\zeta, \phi) \in\left[0, \frac{1}{X-\psi \Delta^{2}}\right] \times\left[0, \frac{X}{\pi\left(\Delta^{1}+\Delta^{2}-\psi \Delta^{2}\right)+X}\right]$ then there is a unique Sequential Equilibrium such that:

$$
\begin{aligned}
& \left(\bar{\lambda}_{1}^{C, 1}, \bar{\lambda}_{1}^{C, 2}, \bar{\lambda}_{1}^{N, 1}, \bar{\lambda}_{1}^{N, 2}\right)=(1,1,0,0) \\
& \rho(\widehat{\delta})=\left\{\begin{array}{ccc}
0 & \text { if } & \left(\widehat{\delta}=\Delta^{1}+\Delta^{2}\right) \\
0 & \text { if } & \left(\widehat{\delta}=\Delta^{1}\right) \\
0 & \text { if } & \left(\widehat{\delta}=\Delta^{2}\right) \\
0 & \text { if } & (\widehat{\delta}=0)
\end{array}\right. \\
& \alpha(\widehat{\delta}, \widehat{\rho})=\left\{\begin{array}{ccc}
0 & \text { if } & \left(\widehat{\delta}=\Delta^{1}+\Delta^{2}, \widehat{\rho}=0\right) \\
0 & \text { if } \quad\left(\widehat{\delta}=\Delta^{1}+\Delta^{2}, \widehat{\rho}=1\right) \\
0 & \text { if } \quad\left(\widehat{\delta}=\Delta^{1}, \widehat{\rho}=0\right) \\
0 & \text { if } \quad\left(\widehat{\delta}=\Delta^{1}, \widehat{\rho}=1\right) \\
0 & \text { if } \quad\left(\widehat{\delta}=\Delta^{2}, \widehat{\rho}=0\right) \\
0 & \text { if } \quad\left(\widehat{\delta}=\Delta^{2}, \widehat{\rho}=1\right) \\
0 & \text { if } \quad(\widehat{\delta}=0, \widehat{\rho}=0) \\
0 & \text { if } \quad(\widehat{\delta}=0, \widehat{\rho}=1)
\end{array}\right. \\
& \mu(C \mid \widehat{\delta}) \in \begin{cases}\{1\} & \widehat{\delta}=\Delta^{1}+\Delta^{2} \\
\{0\} & \widehat{\delta}=\Delta^{2} \\
{[0,1]} & \widehat{\delta}=\Delta^{1} \\
\{0\} & \widehat{\delta}=0\end{cases} \\
& \left(\bar{\lambda}_{2}^{C, 1}, \bar{\lambda}_{2}^{C, 2}, \bar{\lambda}_{2}^{N, 1}, \bar{\lambda}_{2}^{N, 2}\right)=(1,1,0,0)
\end{aligned}
$$


5. if $(\zeta, \phi) \in\left[0, \frac{1}{X-\psi \Delta^{2}}\right] \times\left[\frac{X}{\pi\left(\Delta^{1}+\Delta^{2}\right)+X}, 1\right]$, then with FI there is a unique Sequential Equilibrium such that :

$$
\left(\bar{\lambda}_{1}^{C, 1}, \bar{\lambda}_{1}^{C, 2}, \bar{\lambda}_{1}^{N, 1}, \bar{\lambda}_{1}^{N, 2}\right)=\left\{\begin{array}{l}
(1,1,0,0) \text { with prob. } F I(\psi) \\
(1,1,1,0) \text { with prob. } \operatorname{PE}(\psi) \\
(1,1,1,1) \text { with prob. } \mathrm{FE}(\psi)
\end{array}\right.
$$

where

$i$.

$$
\begin{gathered}
F I(\psi)= \\
=\mathbf{P}\left\{r_{1}^{1} \geq \Delta^{1}+\psi\left[\psi \Delta^{2}+E\left(r_{2}^{1}\right)+E\left(r_{2}^{2}\right)+\frac{X}{\phi}\right] \& r_{1}^{1}+r_{1}^{2} \geq \Delta^{2}+\Delta^{1}+E\left(r_{2}^{1}\right)+E\left(r_{2}^{2}\right)+\frac{X}{\phi}\right\}
\end{gathered}
$$

ii.

$$
\begin{gathered}
P E(\psi)= \\
\mathbf{P}\left\{r_{1}^{1} \leq \Delta^{1}+\psi\left[\psi \Delta^{2}+E\left(r_{2}^{1}\right)+E\left(r_{2}^{2}\right)+\frac{X}{\phi}\right] \& r_{1}^{2} \geq(1-\psi) \Delta^{2}+(1-\psi)\left[\psi \Delta^{2}+E\left(r_{2}^{1}\right)+E\left(r_{2}^{2}\right)+\frac{X}{\phi}\right.\right. \\
=G_{1}\left(\Delta^{1}+\psi\left[\psi \Delta^{2}+E\left(r_{2}^{1}\right)+E\left(r_{2}^{2}\right)+\frac{X}{\phi}\right]\right)\left[1-G_{2}\left((1-\psi)\left[\Delta^{2}+\psi \Delta^{2}+E\left(r_{2}^{1}\right)+E\left(r_{2}^{2}\right)+\frac{X}{\phi}\right]\right)\right]
\end{gathered}
$$

iii.

$$
\begin{aligned}
& F E(\psi)= \\
& =\mathbf{P}\left\{r_{1}^{1}+r_{1}^{2} \leq \Delta^{2}+\Delta^{1}+E\left(r_{2}^{1}\right)+E\left(r_{2}^{2}\right)+\frac{X}{\phi} \& r_{1}^{2} \leq(1-\psi) \Delta^{2}+(1-\psi)\left[\psi \Delta^{2}+E\left(r_{2}^{1}\right)+E\left(r_{2}^{2}\right)+\right.\right. \\
& \text { and } \\
& \begin{array}{c}
\rho(\widehat{\delta})=\left\{\begin{array}{ccc}
0 & \text { if } & \left(\widehat{\delta}=\Delta^{1}+\Delta^{2}\right) \\
1 & \text { if } & \left(\widehat{\delta}=\Delta^{1}\right) \\
1 & \text { if } & \left(\widehat{\delta}=\Delta^{2}\right) \\
1 & \text { if } & (\widehat{\delta}=0)
\end{array}\right. \\
\alpha(\widehat{\delta}, \widehat{\rho})=\left\{\begin{array}{lll}
0 & \text { if } & \left(\widehat{\delta}=\Delta^{1}+\Delta^{2}, \widehat{\rho}=0\right) \\
0 & \text { if } \quad\left(\widehat{\delta}=\Delta^{1}+\Delta^{2}, \widehat{\rho}=1\right) \\
0 & \text { if } \quad\left(\widehat{\delta}=\Delta^{1}, \widehat{\rho}=0\right) \\
0 & \text { if } \quad\left(\widehat{\delta}=\Delta^{1}, \widehat{\rho}=1\right) \\
0 & \text { if } \quad\left(\widehat{\delta}=\Delta^{2}, \widehat{\rho}=0\right) \\
0 & \text { if } \quad\left(\widehat{\delta}=\Delta^{2}, \widehat{\rho}=1\right) \\
0 & \text { if } \quad(\widehat{\delta}=0, \widehat{\rho}=0) \\
0 & \text { if } \quad(\widehat{\delta}=0, \widehat{\rho}=1)
\end{array}\right.
\end{array}
\end{aligned}
$$




$$
\begin{aligned}
\mu(C \mid \widehat{\delta})= & \begin{cases}\frac{\pi}{\pi+(1-\pi)[F E(\psi)+\psi P E(\psi)]} & \widehat{\delta}=\Delta^{1}+\Delta^{2} \\
\frac{0}{0}=0 & \widehat{\delta}=\Delta^{2} \\
0 & \widehat{\delta}=\Delta^{1} \\
0 & \widehat{\delta}=0 .\end{cases} \\
& \left(\bar{\lambda}_{2}^{C, 1}, \bar{\lambda}_{2}^{C, 2}, \bar{\lambda}_{2}^{N, 1}, \bar{\lambda}_{2}^{N, 2}\right)=(1,1,0,0)
\end{aligned}
$$

The outcome in this region is a mixed strategy equilibrium with possible full inefficient, partially and full efficient policy, depending on the probabilities $F I(\psi), P E(\psi)$ and $F E(\psi)$. Moreover we have coups with strictly positive probability, but no revolution in equilibrium.

Some remarks and corollary are useful for the results in the text.

Remarks 1. 1. It is intuitive that

$$
\operatorname{MPE}(0)=0 \text { and } \operatorname{MFE}(1)=0
$$

then

$$
\operatorname{MFE}(0)=\frac{\pi}{1-\pi} \frac{1-\zeta\left(X-\left(\Delta^{1}+\Delta^{2}\right)\right)}{\zeta X-1}>\operatorname{MFE}(1)=0
$$

and

$$
\begin{aligned}
& \operatorname{MPE}(0)=0<\operatorname{MPE}(1)=\frac{\pi}{1-\pi} \frac{1-\zeta\left(X-\left(\Delta^{1}+\Delta^{2}\right)\right)}{\zeta\left(X-\Delta^{2}\right)-1} \\
M F I(0)= & 1-\operatorname{MFE}(0)-\operatorname{MPE}(0)=1-\frac{\pi}{1-\pi} \frac{1-\zeta\left(X-\left(\Delta^{1}+\Delta^{2}\right)\right)}{\zeta X-1}> \\
> & M F I(1)=1-\operatorname{MFE}(1)-\operatorname{MPE}(1)=1-\frac{\pi}{1-\pi} \frac{1-\zeta\left(X-\left(\Delta^{1}+\Delta^{2}\right)\right)}{\zeta\left(X-\Delta^{2}\right)-1} .
\end{aligned}
$$

2. Note that

$$
\begin{gathered}
\frac{d[M F E(\psi)+\psi M P E(\psi)]}{d \psi}=M F E^{\prime}(\psi)+\psi M P E^{\prime}(\psi)+M P E(\psi)=\frac{\pi}{1-\pi} \frac{\zeta \Delta^{2}\left[1-\zeta\left(X-\left(\Delta^{1}+\Delta^{2}\right)\right)\right]}{\left[\zeta\left(X-\psi \Delta^{2}\right)-1\right]^{2}} \geq 0 \\
\frac{d M F I(\psi)}{d \psi}=-\frac{d[M F E(\psi)+M P E(\psi)]}{d \psi}=-M F E^{\prime}(\psi)-M P E^{\prime}(\psi)
\end{gathered}
$$

Moreover

$$
\left.\frac{d[M F E(\psi)+\psi M P E(\psi)]}{d \psi}\right|_{\psi=0}=M F E^{\prime}(0)+M P E(0)=0 \Leftrightarrow M F E^{\prime}(0)=0
$$

and

$$
\left.\frac{d[M F E(\psi)+\psi M P E(\psi)]}{d \psi}\right|_{\psi=1}=M F E^{\prime}(1)+M P E^{\prime}(1)+M P E(1)=\frac{\pi}{1-\pi} \frac{\zeta \Delta^{2}\left[1-\zeta\left(X-\left(\Delta^{1}+\Delta^{2}\right)\right)\right]}{\left[\zeta\left(X-\Delta^{2}\right)-1\right]^{2}} \Leftrightarrow
$$




$$
\begin{gathered}
\Leftrightarrow M F I^{\prime}(1)=-\left[M F E^{\prime}(1)+M P E^{\prime}(1)\right]=-\left\{\frac{\pi}{1-\pi} \frac{\zeta \Delta^{2}\left[1-\zeta\left(X-\left(\Delta^{1}+\Delta^{2}\right)\right)\right]}{\left[\zeta\left(X-\Delta^{2}\right)-1\right]^{2}}-M P E(1)\right\}= \\
=-\frac{\pi}{1-\pi} \frac{\left[1-\zeta\left(X-\left(\Delta^{1}+\Delta^{2}\right)\right)\right]\left[1-\zeta\left(X-2 \Delta^{2}\right)\right]}{\left[\zeta\left(X-\Delta^{2}\right)-1\right]^{2}} \leq 0 \text { if } \Delta^{1} \geq \Delta^{2}
\end{gathered}
$$

These results and the simulations corroborates our conjecture that

$$
M F E^{\prime}(\psi) \leq 0, \quad M P E^{\prime}(\psi) \geq 0, \quad M F I^{\prime}(\psi) \leq 0 .
$$

3. Using simulation, is possible to confirm the intuition that

$$
\frac{d \bar{\alpha}}{d \psi} \geq 0
$$

The reason is that

$$
\begin{gathered}
\mu\left(C \mid \widehat{\delta}=\Delta^{1}+\Delta^{2}\right)=\frac{X-(\zeta)^{-1}-\psi \Delta^{2}}{\Delta^{1}+\Delta^{2}-\psi \Delta^{2}} \Rightarrow \\
\frac{\partial \mu\left(C \mid \widehat{\delta}=\Delta^{1}+\Delta^{2}\right)}{\partial \psi} \leq 0 \Leftrightarrow \zeta \leq \frac{1}{X-\left(\Delta^{1}+\Delta^{2}\right)}
\end{gathered}
$$

i.e. as $\psi$ increases, then the belief of facing a congruent leader is reducing and therefore a higher probability of revolt is required to induce a (partially) efficient policy.

Corollary 4. 1. the probability of a Partially Efficient Regime PE $(\psi)$ is increasing in opacity $\psi$ but bounded away from one, and it disappears when there is full transparency, i.e. $\psi=0$;

2. the probability of a Roving Bandit Regime FI $(\psi)$ is decreasing in opacity $\psi$ but bounded away from zero with full opacity, i.e. $\psi=1$, and from one when there is full transparency, i.e. $\psi=0$;

3. the probability of a Full Efficient Regime FE $(\psi)$ is decreasing in opacity $\psi$ and it disappears with full opacity, i.e. $\psi=1$, and from one when there is full transparency, i.e. $\psi=0$.

Proof. From proposition 1 the behavior of $P E(\psi), F I(\psi)$ and $F E(\psi)$ is immediate, moreover

$$
\begin{gathered}
F I(0)=1-G\left(r_{1}^{1} \geq \Delta^{1} \& r_{1}^{1}+r_{1}^{2} \geq \Delta^{2}+\Delta^{1}+E\left(r_{2}^{1}\right)+E\left(r_{2}^{2}\right)+\frac{X}{\phi}\right) \\
F I(1)=1-G_{1}\left(r_{1}^{1} \geq \Delta^{1}+\Delta^{2}+E\left(r_{2}^{1}\right)+E\left(r_{2}^{2}\right)+\frac{X}{\phi}\right) \\
P E(\psi)=G_{1}\left(\Delta^{1}+\psi\left[\psi \Delta^{2}+E\left(r_{2}^{1}\right)+E\left(r_{2}^{2}\right)+\frac{X}{\phi}\right]\right)\left[1-G_{2}\left((1-\psi)\left[\Delta^{2}+\psi \Delta^{2}+E\left(r_{2}^{1}\right)+E\left(r_{2}^{2}\right)+\frac{X}{\phi}\right]\right)\right]
\end{gathered}
$$




$$
\begin{gathered}
P E(0)=0 \text { and } P E(1)=G_{1}\left(\Delta^{1}+\Delta^{2}+E\left(r_{2}^{1}\right)+E\left(r_{2}^{2}\right)+\frac{X}{\phi}\right) \\
F E(0)=G\left(r_{1}^{1}+r_{1}^{2} \leq \Delta^{2}+\Delta^{1}+E\left(r_{2}^{1}\right)+E\left(r_{2}^{2}\right)+\frac{X}{\phi}\right) \text { and } F E(1)=0 .
\end{gathered}
$$

Consider in details the outcomes associated to the above SE. In particular we will show how a single equilibrium is associated to multiple outcomes with well defined probabilities. There are four possible equilibrium outcomes when we consider the non congruent leader policy choices and the consequent reaction by selectorate and by citizens:

1. a Stationary Bandit outcome, where the leader's inefficient policies don't trigger any reaction by the selectorate and the citizens;

2. a Roving Bandit outcome, where the leader's inefficient policies trigger a coup by the selectorate and/or a revolt by the citizens;

3. a Partially Efficient outcome, where the leader choose an efficient policy only on the full observable policy, risking a coup by the selectorate and/or a revolt by the citizens because of the inefficient choice on the partially observable policy;

4. a Full Efficient outcome, where the leader choose an efficient policy on both dimensions, to minimize the risk of a coup by the selectorate and/or a revolt by the citizens.

Related to these four possible equilibrium outcomes, there are four possible political autocratic regimes with different observable characteristics.

We sum up outcomes and political regimes in the following proposition.

Proposition 2. 1. If

$$
(\zeta, \phi) \in\left[0, \frac{1}{X-\psi \Delta^{2}}\right] \times\left[0, \frac{X}{\pi\left(\Delta^{1}+\Delta^{2}-\psi \Delta^{2}\right)+X}\right]
$$

then there is a Kleptocratic Autocracy (KA) characterized by a unique Stationary Bandit outcome with:

- full inefficient policy $\left(\bar{\lambda}_{1}^{N, 1}, \bar{\lambda}_{1}^{N, 2}\right)^{*}=(0,0)$,

- no coup $\rho^{*}=0$,

- no revolution $\alpha^{*}=0$;

1. if

$(\zeta, \phi) \in\left[\frac{1}{X-\psi \Delta^{2}}, \frac{1}{X-\left[\pi\left(\Delta^{1}+\Delta^{2}\right)+(1-\pi) \psi \Delta^{2}\right]}\right] \times[0,1] \cup\left[0, \frac{1}{X-\psi \Delta^{2}}\right] \times\left[\frac{X}{\pi\left(\Delta^{1}+\Delta^{2}\right)+X}, 1\right]$ then there is a Semi Efficient Autocracy (SEA) characterized by three possible outcomes: 
i. a Roving Bandit outcome with probability FI $(\psi)$ with:

- full inefficient policy $\left(\bar{\lambda}_{1}^{N, 1}, \bar{\lambda}_{1}^{N, 2}\right)^{*}=(0,0)$,

- $\operatorname{coup} \rho^{*}=1$,

- no revolution $\alpha^{*}=0$;

ii. a Partially Efficient outcome with probability PE $(\psi)$ with:

- partially efficient policy $\left(\bar{\lambda}_{1}^{N, 1}, \bar{\lambda}_{1}^{N, 2}\right)^{*}=(1,0)$,

- possible coups $\rho^{*} \in\{0,1\}$ with probability $\rho=1-\psi$,

- no revolution $\alpha^{*}=0$;

iii. a Fully Efficient outcome with probability $F E(\psi)$ with:

- fully efficient policy $\left(\bar{\lambda}_{1}^{N, 1}, \bar{\lambda}_{1}^{N, 2}\right)^{*}=(1,1)$,

- $\operatorname{no~} \operatorname{coup} \rho^{*}=0$,

- no revolution $\alpha^{*}=0$;

2. if

$$
(\zeta, \phi) \in\left[\frac{1}{X-\left[\pi\left(\Delta^{1}+\Delta^{2}\right)+(1-\pi) \psi \Delta^{2}\right]}, \frac{1}{X-\left(\Delta^{1}+\Delta^{2}\right)}\right] \times[0,1],
$$

then there is a Mixed Semi Efficient Autocracy (MSEA) characterized by three possible outcomes:

i. a Roving Bandit outcome with probability MFI $(\psi)$ with:

- full inefficient policy $\left(\bar{\lambda}_{1}^{N, 1}, \bar{\lambda}_{1}^{N, 2}\right)^{*}=(0,0)$,

- possible coups $\rho^{*} \in\{0,1\}$ with probability $\bar{\rho} \in(0,1)$,

- revolution $\alpha^{*}=1$;

ii. a Partially Efficient outcome with probability $M P E(\psi)$ with:

- partially efficient policy $\left(\bar{\lambda}_{1}^{N, 1}, \bar{\lambda}_{1}^{N, 2}\right)^{*}=(1,0)$,

- possible coups $\rho^{*} \in\{0,1\}$ with probability $\widetilde{\rho}=(1-\psi) \bar{\rho} \in(0,1)$,

- possible revolution $\alpha^{*} \in\{0,1\}$ with probability $\widetilde{\alpha}=(1-\psi)+\psi \bar{\alpha} \in(0,1)$;

iii. a Fully Efficient outcome with probability $M F E(\psi)$ with:

- fully efficient policy $\left(\bar{\lambda}_{1}^{N, 1}, \bar{\lambda}_{1}^{N, 2}\right)^{*}=(1,1)$,

- $\operatorname{no~coup~} \rho^{*} \in\{0\}$,

- possible revolution $\alpha^{*} \in\{0,1\}$ with probability $\bar{\alpha} \in(0,1)$;

3. if

$$
(\zeta, \phi) \in\left[\frac{1}{X-\left(\Delta^{1}+\Delta^{2}\right)}, 1\right] \times[0,1],
$$

there is a Failed State Autocracy (FSA) characterized by a unique Roving Bandit outcome with: 
- full inefficient policy $\left(\bar{\lambda}_{1}^{N, 1}, \bar{\lambda}_{1}^{N, 2}\right)^{*}=(0,0)$,

- possible coup $\rho^{*} \in\{0,1\}$ with probability $\bar{\rho} \in[0,1]$,

- revolution $\alpha^{*} \in\{1\}$.

Proof. The proposition follows immediately from Proposition 1. 